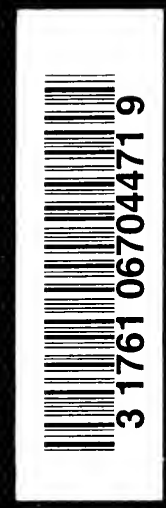


YHE J. F. HARTZ CO LIMITEO

MEDICAL BOOKB 


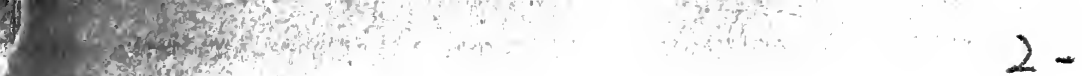

(5)

$2-$ M Thents

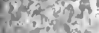

$3+x+(4)^{2}=$

$+x x^{2}+3$

$2 \geqslant 3 y^{2}-3$

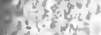

his

:-itivi i

$x^{2}+2$

$2 y^{2}$,

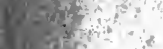

atogen

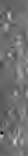

$\frac{1}{10}$

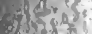

the

4

ats?

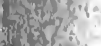

at?

Q 


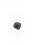




\title{
DISEASES of CHILDREN
}

\author{
FOR \\ NURSES
}

INCLUDING

INFANT FEEDING, THERAPEUTIC MEASURES EMPLOYED IN CHILDHOOD, TREATMENT FOR EMERGENCIES, PROPHYLAXIS, HYGIENE, AND NURSING

\section{BY}

ROBERT S. MCCOMBS, M. D.

Assistant Physician to the Dispensary and Instructor of Nurses at the Children's Hospital of Philadelphia

ILLUSTRATED

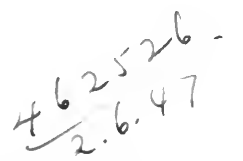

SECOND EDITION, THOROUGHLY REVISED

PHILADELPHIA AND LONDON

W. B. SAUNDERS COMPANY 1912 
Copyright, 1907, by W. B. Saunders Company. Revised, reprinted, and recop'righted, January, rgxi.

Copyright, rgı, by W. B. Saunders Company.

Reprinted September, 1912.

\section{PaINTEO IN AMERICA}

PRE 88 OF

W. 8. SaUNDERS COMPANY

PHILADELPHIA 


\section{DEDICATED}

TO

THE NURSES OF THE TRAINING SCHOOL

OF

THE CHILDREN'S HOSPITAL OF PHILADELPHIA. 
, 


\section{PREFACE TO THE SECOND EDITION.}

THE author wishes to thank the reviewers for their almost universal approval of the first edition of this book.

In the present edition the text has been thoroughly revised and brought up to date. The chapters on intestinal diseases and therapeutics have been amplified.

The chapter on artificial feeding has been improved by the addition of data, especially in reference to topmilk mixtures. Numerous practical points in the nursing of children's diseases have been incorporated.

The author again wishes to thank Miss Jennie A. Manly, head nurse of the Children's Hospital of Philadelphia, for her advice and help in revising the articles on nursing.

He hopes this second edition will be as favorably received as the previous one, and that the nurse may find it a thoroughly satisfactory guide in her management of sick children.

To the publishers he expresses his sincere appreciation of the painstaking care bestowed upon every detail of the volume, and thanks them for many acts of courtesy.

Robert S. McCombs.

I3o South Twenty-SEcond Street,

Philadelphia. 


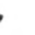




\section{PREFACE}

THE need for a book on children's diseases for nurses was called to the author's attention while giving a course of lectures to the nurses at The Children's Hospital of Philadelphia.

It was his original intention to have only the notes of the lectures printed, so that some form of condensed material might be at hand for purposes of study. This volume has grown from the original notes. Incorporated in it are the methods employed at the Children's Hospital. A short description of each disease found in infancy and childhood has been given. It is hoped that the descriptions are clear enough to enable a nurse to know what symptoms to expect and what complications to guard against. The author believes that enough anatomy and pathology have been included to give a clear understanding of the structure of the body and the changes which take place during disease. Treatment has been included where a thorough knowledge of its underlying reason is necessary for intelligent application. Treatment for emergencies will be found under the different diseases and in the chapter on Therapeutics. Prophylaxis, infant feeding, and the methods of nursing employed in childhood are dwelt upon. 
The author is most deeply indebted to Miss Jennie A. ManLY for practically all the points on nursing, together with the receipts for infant feeding used in this book.

The original photographs for this volume were taken at the Children's Hospitai.

In compiling the notes, the author has had frequent recourse to most of the text-books on children's diseases. For many definitions throughout the book, and the description of the diseases of the kidney, he has consulted Stevens' Manual of Medicine. In the chapters on Gastro-intestinal Diseases and Infant Feeding, Holt's Infancy and Childhood was often consulted.

Robert S. MCСombs. 


\section{CONTENTS}

CHAPTER I

PAGE

Peculiarities of Children's Diseases . . . . . . . . . . I I

CHAPTER II

NuRsing in Childhood . . . . . . . . . . . . 29

CHAPTER III

Diseases of the Respiratory Tract . . . . . . . . . 46

CHAPTER IV

Diseases of the Respiratory Tract (continued) . . . . 8 i

CHAPTER V

Diseases of the Digestive Tract . . . . . . . . . io i

CHAPTER VI

Diseases of the Digestive Tract (continued). . . . . i 18

CHAPTER VII

Diseases of the Digestive Tract (continued). . . . . I 27

CHAPTER VIII

Diseases of the Circulatory System . . . . . . . . I55

CHAPTER IX

Nervous Diseases . . . . . . . . . . . . . I I 79

CHAPTER $\mathrm{X}$

Diseases of the URINARY Tract . . . . . . . . 209 


\section{CHAPTER XI}

Diseases of the Eye, Ear, Skin, and Glandular System Pagx

CHAPTER XII

The Infectious Fevers 242

CHAPTER XIII

TYPHOID FEVER . . . . . . . . . . . . . . . 258

CHAPTER XIV

TUberculosis . . . . . . . . . . . . . . . . $27^{I}$

CHAP'TER XV

Contagious Diseases . . . . . . . . . . . . . . 284

CHAPTER XVI

Constitutional and Nutritional Diseases .

CHAPTER XVII

INFANT FEEDING . . . . . . . . . . . . . . 328

CHAPTER XVIII

Artificial Feeding . . . . . . . . . . . . . 348

CHAP'TER XIX

Therapeutics . . . . . . . . . . . . . . . . 377

CHAPTER XX

Weights and Measures; Abbreviations.. . . . . . . 439 CHAPTER XXI

Medical Terminology . . . . . . . . . . . . 445

INDEX ...................... . . . 457 


\section{Diseases of Children for Nurses}

\section{CHAP'TER I}

\section{PECULIARITIES OF CHILDREN'S DISEASES}

A CHILD's life is divided into definite periods, namely, the "newborn," "infancy," "childhood," and "youth."

The Newborn.-It is customary to designate a babe as "newborn" until all traces of its prenatal or intra-uterine existence have disappeared. This is usually accomplished by the end of the first month.

Infancy follows the newborn period and continues until the eruption of the first or milk teeth is completed; it is, therefore, limited to the first three years of life.

Childhood extends from the third to the seventh year, at which time the permanent teeth make their appearance.

Youth includes the years from seven to puberty.

Weight.-The normal infant should weigh about seven and a half pounds at birth. During the first week there is a slight loss, but from that time on the increase should be steady.

The curve during the first year is represented in the chart and shows approximately the proper weight for the different ages of the infant.

Height.-The average height is about twenty and a 


\section{INFANT'S WEIGHT CHART.}

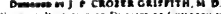

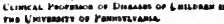

Date of Birth,

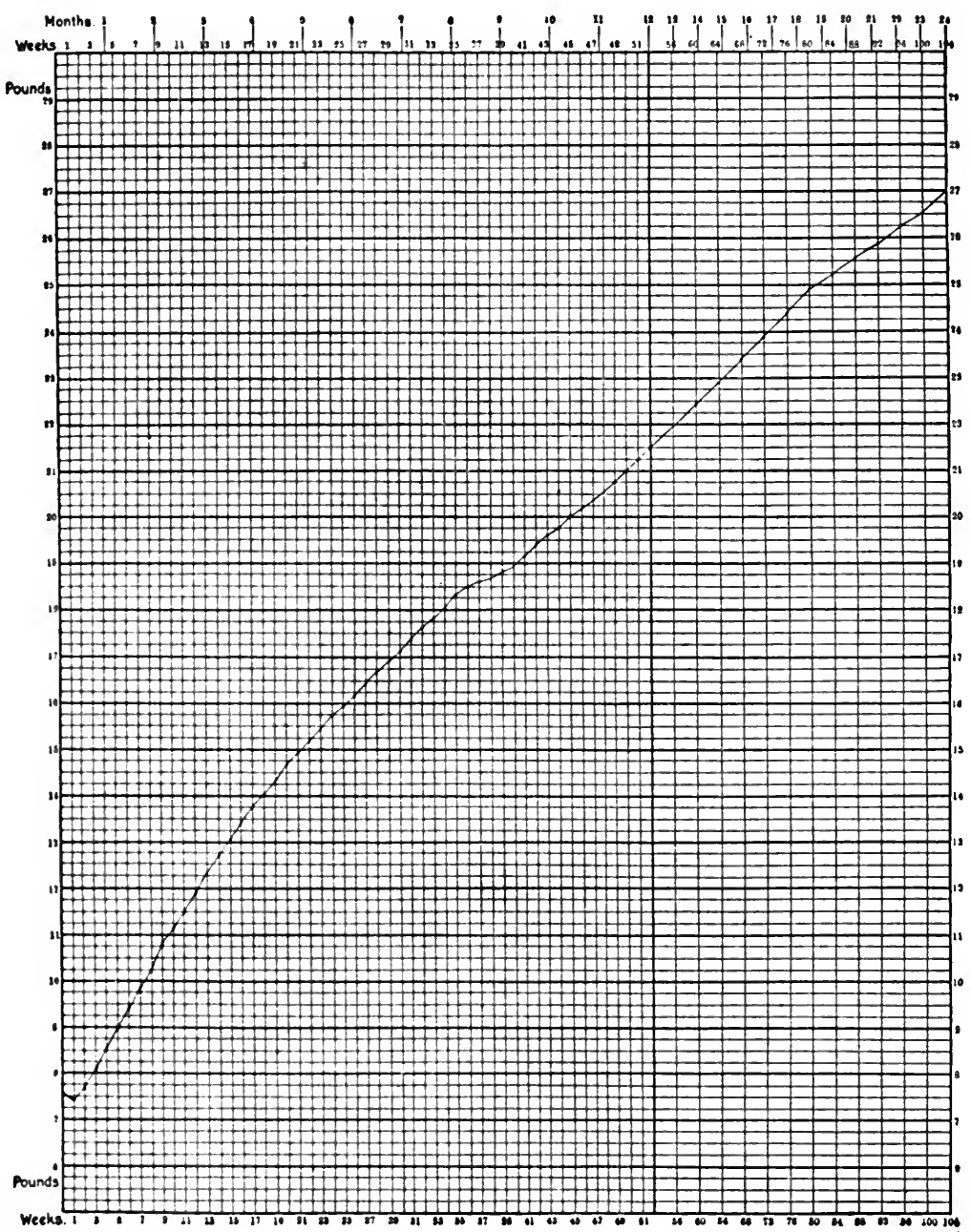

Fir.. 1. 
Weight Chars

NAME,

ADM.

AGE,

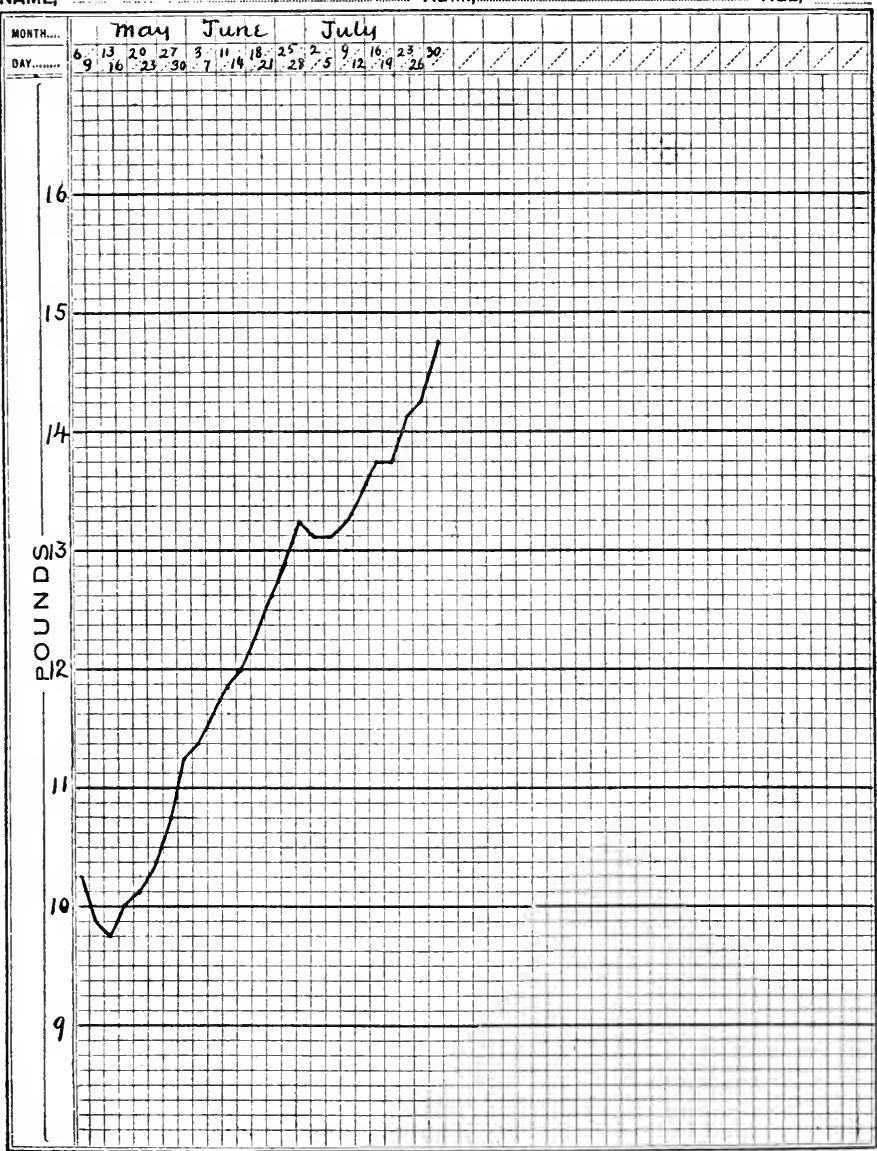

Fig. 2.-The above chart was designed by Miss Rena C. Fox. Head Nurse, Children's Department of Philadelphia Hospital. It is very useful in cases where it is desirable to keep account of a child's weight over a short periol of time. In the larger charts a month's time would appear as a very small line. The heavy lines may represent any pound desired; the figures being given according to first weight of child during reriod it is to be used. Each subdivision equals two ounces. 
half inches at birth. The growth during the first year is about eight inches.

The growth of the extremities is much more rapid than that of the trunk. The head measures in circumference about thirteen and a half inches. The posterior fontanels should close at the end of the second month and the anterior about the eighteenth month.

The special senses of sight, hearing, smell, and touch are developed at birth.

A normal infant is able to hold up its head during the second month and sit up about the sixth month. It begins to recognize objects during the fifth month. From the eighth to the sixteenth month it should learn to walk. The age at which an infant begins to talk varies greatly. In the majority of cases by the end of the first year it is able to form certain words, and from this time on the development of the function of speech is rapid. At times perfectly healthy children have made little progress in their ability to talk by the end of the second year.

Teeth.-Average table of eruption of the milk teeth:

Centrals.............. 5 to 8 months.

Latcrals............. 7 to 10 "

First molar..............

Cuspid................... I4 to 25 "

Second molar.............24 to 36 "

At threc years all of the milk teeth should be in place.

The lower teeth usually erupt first.

The milk teeth demand the same attention as the permanent teeth.

Average table of eruption of the permanent teeth: 
First molar......... 5 to 7 years.

Centrals.............6 6 to 8 "

Laterals........... 7 to 8 "

First bicuspid........ 9 to Io

Second bicuspid......... Io to I2

Cuspid.............. I 2 to 13

Second molar.......... I 2 to I4

Third molar.......... 6 to 35 , to 40 years.

In strumous or rickety children the eruption is frequently delayed and the teeth are often brittle and fur-
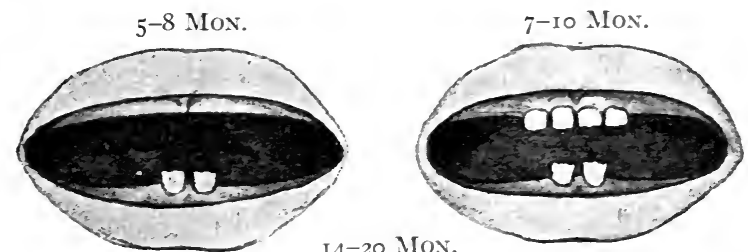

14-20 MoN.

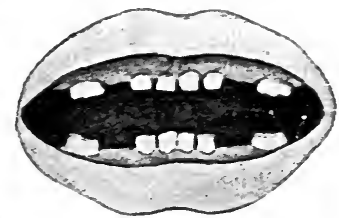

I4-25 MoN.
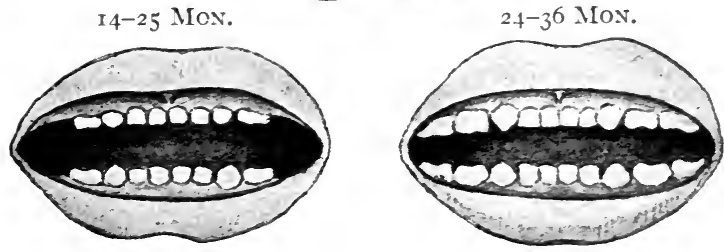

FIG. 3.- Normal dentition (Frühwald and Westcott).

rowed. The enamel may be of poor quality, so that they easily decay. 
Hutchinson's teeth are seen in hereditary syphilis. The second or permanent upper central incisors have a single shallow crescentic notch in the center of the edge. In addition the teeth are small and pegged. They are sometimes called "screw-driver" teeth.

Laminated and pitted teeth are seen at times after the acute infectious discases of childhood, such as measles,
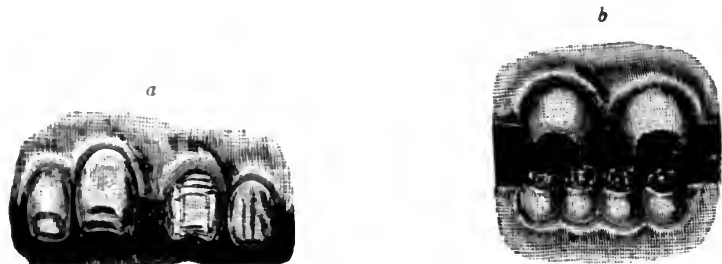

FIG. 4.-Hutchinson's teeth in hereditary syphilis: $a$, The two upper central incisors (second dentition) exhibit deep transverse and longitudinal furrows and a concave notch in the edge. Although the teeth are normal in length, the width is less than normal, thus producing a broad interspace between the central incisors; $b$, the upper central incisors (second dentition), immediately after eruption, and the four lower incisors. The lower surface of the upper incisors is rough from the presence of projecting points of dentine. The upper teeth are short and diverge, leaving a broad interval between them. The four lower incisors present a number of small excrescences like nails from imperfect enamel formation. The base of the excrescences is everywhere in the same plane (aiter Hutchinson).

scarlet fever, and diphtheria. When these diseases occur at an early age the formation of the enamel may be affected. 'This causes such defects as irregular pits upon the crowns of the teeth, particularly the incisors. The pitting is so marked in some cases that it gives a general honeycombed appearance to the crowns. To ascertain if irregularities are due to the eruptive fevers it is necessary to know the age of the child at the period of the disease. Pits upon the incisors caused by the eruptive fevers between the ages of four and five occupy about the central area of the crown face. The enamel about the cutting edge of 
the teeth has already formed at this age, so that alterations of nutrition would not affect it. The crowns of all the

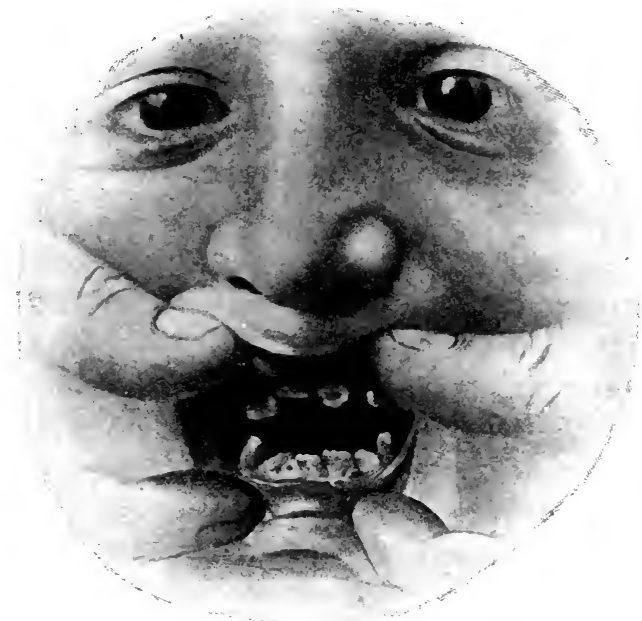

FIG. 5.-Rachitic teeth. Boy nine and a half years old. The teeth are poorly developed, considerably eroded and grooved. Their position is very irregular; the lower incisors occupy a frontal position (not in the arch of the jaw) and the inferior maxilla makes an angular turn at the canine teeth (Hecker, Trumpp, and Abt).

teeth in process of formation at this age are affected in a similar manner.

Diseases Peculiar to Children.-Children need different environments, different management, different medical and surgical care, and different nursing from that required for adults. One-third of a physician's patients are children.

Diseases of infancy and of early childhood differ in many respects from those of adult life, but after the seventh year children resemble adults in their ailments more than they do infants. 
The following diseases are seen chiefly in infancy and childhood: congenital anomalies of the heart, such as "blue babies," due to a patulous foramen ovale; congenital atelectasis or failure of a part of a lung to expand; ophthalmia neonatorum or a gonorrheal conjunctivitis in the newborn; traumatic hemorrhages and birth paralysis due to injuries during birth; umbilical hernia; noma or gangrenous stomatitis; cholera infantum; laryngismus stridulus; enuresis; chorea; tubercular meningitis; hydrocephalus; infantile cerebral and spinal paralysis; coxalgia; scarlet fever; measles; rubella; varicella; pertussis; mumps, and diphtheria.

Etiology.-Heredity, accidents at birth, infection through the umbilical cord, improper food, and bad hygienic surroundings are the principal causes of disease or of a delicate constitution in the newborn and during infancy.

Inheritance is a very strong factor in infancy. Such diseases as syphilis and tuberculosis can be directly transmitted from the mother to the child. Syphilis very frequently is congenital. While a child is rarely born with tuberculosis, the undermined constitution and the fecble resisting power of an infant born of tubercular parents make it very likely that the disease will develop early in life, at times so soon after birth as to be practically congenital. This is especially so if they breathe the same atmosphere as, or are nursed by, tubercular mothers. Children of parents suffering with rheumatism, gout, Bright's disease, or alcoholism are apt to have poor resisting powers and yield easily to infection.

Accidents at birth give rise to conditions in the newborn such as birth palsies, hemorrhages of the meninges or 
membrane covering the brain and spinal cord. Infection through the umbilical cord may cause pyemia, this condition being characterized by the formation of abscesses in the various organs.

Improper food and bad hygiene are the greatest causative factors of disease in infancy. To these are due not only such diseases as rickets, scurvy, and marasmus, but also the great class of gastro-intestinal disorders.

The Most Frequent Diseases of Infancy and Childhood.-Diseases of the gastro-intestinal tract and bronchopneumonia are seen more often than any other diseases before the second year. The other common conditions met with during this period are affections of the lymphglands, tubercular meningitis, pertussis, and measles.

After the second year the following diseases are most frequently seen: Disorders of nutrition, such as rickets and scurvy; bone and joint diseases, these being usually tubercular and more rarely syphilitic; diseases of the blood; organic diseases of the heart; pneumonia, typhoid fever, the acute contagious diseases, such as measles, mumps, pertussis, varicella, scarlet fever, and diphtheria.

\section{COMPARISON OF CHILDREN'S DISEASES WITH ADULT CONDITIONS}

The respiratory tract during infancy is undeveloped. The air-cells in the lungs are not so far advanced in their structure, nor so important in the function of respiration, as are the bronchial tubes. This causes an ordinary inflammation of the bronchial tubes, or bronchitis, to be a much more serious condition in infancy than in the adult. When the smallest tubes are involved it is called capillary bronchitis or bronchopneumonia. 
Pneumonia is very common in infancy and childhood. Before two years of age it is usually of the type of a bronchopneumonia. Next to gastro-intestinal disease this form of pneumonia causes more deaths than any other condition during childhood. After two years of age the pneumonia is usually croupous in type, and, in contradistinction to bronchopneumonia, is very rarely fatal. In this respect it differs from pneumonia in the adult, in whom the mortality ranges from 20 per cent. to 50 per cent. The frequency of empyema as a complication of pneumonia and the frequency of bronchopneumonia as a complication of the acute infectious fevers are peculiarities of children's diseases.

Gastro-intestinal disorders in childhood are the most fatal of all diseases. This is in direct contrast to adult life. The reason lies in the delicate digestive power of infants. Toxins or poisons are formed in the intestines from their inability to digest and assimilate properly the food given. This poison is absorbed and produces grave results. During infancy the stomach and intestines are more intimately associated than later in childhood. Hence one is rarely affected without involving the other. This causes an increase in the severity of the symptoms, with consequent deleterious results to the child. Throughout childhood gastro-intestinal conditions are characterized by the severity of the symptoms.

The circulatory tract, except for the congenital malformations of the heart such as valvular disease and "blue babies," is usually unaffected during infancy. After two years of age heart diseases are common. A murmur should always be looked for in cases of chorea, rheumatism, and the acute contagious diseases. A peculiarity of 
childhood is the development of endocarditis following mild attacks of rheumatism. Aneurysms and arteriosclerosis are uncommon.

Anemia is often present in children.

The Genito-urinary Tract.-The kidneys are rarely affected in childhood except as a sequel of scarlet fever. As this is a very common complication of scarlet fever, all those suffering from the disease should be kept under close observation.

Enuresis is common in childhood.

The various malformations of this tract are common in childhood, especially phimosis.

Diseases of the Nervous System.-Hemorrhages are usually on the surface of the brain, and not within its substance. They usually occur at birth. Birth paralysis results. The portion of the cortex at which such hemorrhages occur fails to develop, and the injury is permanent.

Such conditions as chorea (St. Vitus' dance), laryngismus stridulus, nodding spasms, tetany, nystagmus, infantile paralysis, and tubercular meningitis are typically children's diseases.

One of the peculiarities of children is to have convulsions from trivial causes, without any lesion of the brain being present.

Diseases of the Eyes, Ears, Skin, and Glandular System.-Ophthalmia in the newborn is very often seen in the poorer classes and causes about 60 per cent. of congenital blindness. Conjunctivitis is often seen, especially associated with measles and catarrhal conditions of the nose and throat. Strabismus is not uncommon.

Otitis media and mastoid disease are more frequent in childhood than in the adult. Running ears, following 
the contagious diseases, especially measles, is a common complication. Eczema is often observed in artificially fed children.

Tubercular adenitis is seen more often in childhood than in the adult.

The Acute Infectious Fevers.-Most of the diseases seen in adults attack children. Malaria is common. Hereditary syphilis is observed in childhood, the majority dying before they reach maturity.

Typhoid feicr is quite a common disease in children living in large cities. It differs from typhoid fever in the adult in that its onset is usually more sudden, manifesting itself as often in the appearance of fever, romiting, and prostration as in the usual slow, insidious beginning. In the course of the disease constipation is more frequent than diarrhea, tympanites is not so marked, the eruption is less constant, the nervous symptoms are not as apt to be found as in adults, hemorrhage and perforation are also met with less often, and the mortality is lower.

Tuberculosis is common in childhood. In infants under two years of age the lung is the part affected; beginning with the second year tubercular meningitis is more often found; and after the third year tuberculosis of the bones, the lymph-glands, peritoneum, and intestines becomes more frequent and are seen throughout childhood. Pott's disease and coxalgia are rarely seen except in childhood.

The contugious disease's are typically children's diseases. While they may attack unprotected adults, they are comparatively rare after fifteen years of age.

Constitutional and Nutritional Diseases.-Rheumatism is rarely of the acute articular type in children. It is often exhibited only by stifiness and slight aching pains 
in the limbs ("growing pains"). The frequency of endocarditis as a complication of rheumatism, even when no more severe symptoms than growing pains are present, makes it necessary that such cases should be put to bed and receive proper treatment.

Diabetes mellitus is uncommon in childhood, but when it does occur the course is very rapid and fatal.

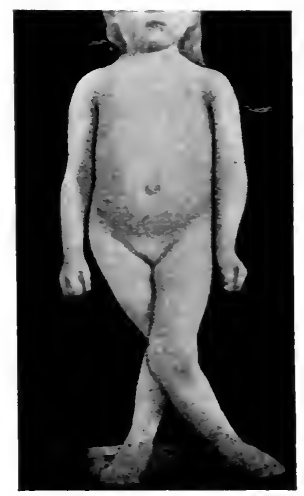

FIG. 6.-Genu valgum. Female child five years old (Napier).

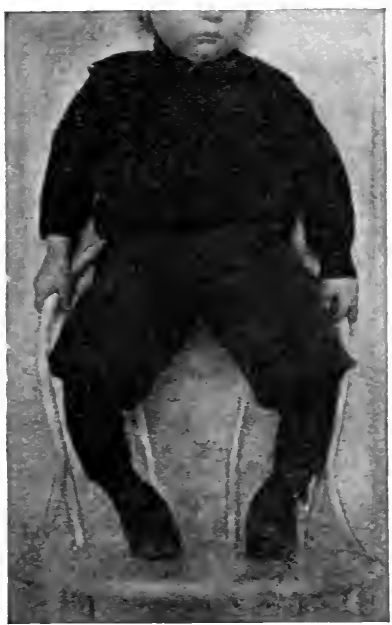

FIG. 7.-Genu varum from rachitis (Napier).

Scurvy is seen in artificially fed infants.

Rickets and marasmus are typical nutritional diseases seen only in childhood.

Orthopedics.-Many children have deformities due to rickets, spinal curratures, congenital dislocation of the hips, and contractions of the tendons. These deformities are overcome by surgical treatment. 
Various terms are given to the different deformities:

Clubbed hands; webbed fingers; congenital dislocation of the hips; knock-knees or genu valgum; bow-legs or genu varum; bowing of tibia; club-feet or talipes varus; and talipes valgus; polydactyly or six fingers.

Symptomatology.--In children the onset of disease is usually more sudden, the temperature higher, the pulse and respirations more accelerated, the physical signs more pronounced, the course shorter, and the recovery more rapid than in an adult.

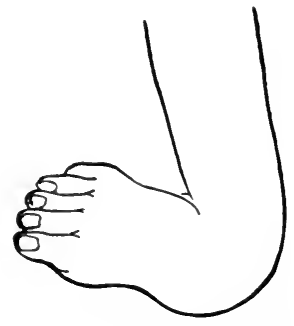

Frg. 8.-Talipes valgus (Kerr).

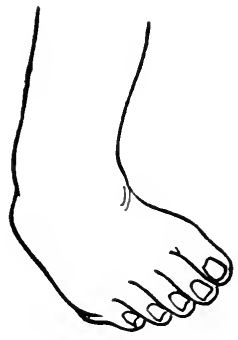

FIG. 9. - Talipes varus (Kerr).

All temperatures in children are higher than in adult life. Frequently a temperature of $104^{\circ} \mathrm{F}$. to $105^{\circ} \mathrm{F}$. is seen in cases of ordinary pharyngitis and mild tonsillitis. Fever in children apparently results from the slightest cause. This is better understood if one realize that the nervous mechanism of a child is more sensitive than that of an adult. Consequently a fever in a child does not indicate as much as in an adult and need not cause anxiety unless prolonged. It is the continuous high temperature which indicates serious illness. On the other hand, the temperature is easily depressed, owing to the great vascularity of the skin, by exposure, by sleep, and by inactivity. 
Inanition fever is a term applied to a peculiar elevation of temperature occurring in the newborn. It is generally seen during the first five days of life, and is apparently due to the fact that the infant gets very little, often nothing at all, from the breast. The temperature may rise to IO2 ${ }^{\circ}$ or $104^{\circ} \mathrm{F}$., and is associated with rapid loss of weight. As soon as milk is secreted in abundance, or when the child is placed upon a full breast, artificial food, or even water, if given freely, the temperature falls to normal. It is important that such a fever should be recognized, because it gives at times the first warning of a condition which may prove fatal. The temperature of every child should be taken during the first week.

The normal pulse in infancy and childhood is of lower tension than in an adult and varies in frequency according to the age. The first few weeks after birth it beats from I 20 to I5O times per minute. In the second year the pulse falls to Iro; in the third or fourth year to Ioo; in the seventh to go; and in the twelfth to 8o. Slight causes may produce wide variations of the normal pulse due to the unstable nervous mechanism of a child.

The respirations during the same period likewise varyIn the newborn they are from 30 to 60 per minute; in the first year 28 to 30 ; at five years 22 to 25 ; at fourteen years 20; and in adult life 18 . The peculiar variations of the respiration seen in childhood are due to the same nervous origin which influences the temperature and pulse.

The function of digestion in infancy is delicate and undeveloped. Infants are able to digest about 4 per cent. of fat, 6 per cent. of sugar, and 2 per cent. of proteid during the first year.

Infants cannot tell their symptoms, so it is necessary to 
study them to find out their ailments. The principal means a child has of explaining its wants, discomforts, or pains is by crying. A child cries from pain, hunger, discomfort, or habit.

The cry of hunger is usually fretful, is accompanied by the sucking of its fingers, and ceases when satisfied.

The cry of indigestion simulates the cry of hunger, but does not cease when the child is fed.

The cry of pain is usuaily sharp and is accompanied by contractions of the features, drawing up of the legs, and signs of distress. If the child fall asleep from exhaustion it soon awakens, usually with a scream. It is well to remember that a severe pain in infancy may be due either to colic or earache. The child simply moans when the pain is less severe.

The cry of reakness is a feeble whine.

The cry of temper is prolonged, violent, and is attended with stiffness of the body and the throwing about of the arms and legs.

The cry of habit ceases when the child is satisfied and may be caused by a desire for any familiar object, such as a doll, nipple, or rattle.

There are also characteristic cries heard in certain diseases such as hydrocephalus, meningitis, marasmus, hereditary syphilis, and pneumonia.

In hydrocephalus and meningitis a child will scream out shrilly in the night. This is called the hydrocephalic cry and is also sometimes heard in chronic bone diseases, due to pain.

In marasmus there is the feeble whine; in hereditary syphilis a nasal cry; in pneumonia the cry is short, catching, and suppressed. 
Feeding and Therapeutics.-The entire subject of feeding in infancy and childhood is one of the most important branches of pediatrics.

The therapeutic measures employed differ in many respects from adult treatment.

Prognosis.-The younger the child, the worse the prognosis. This is because of the feeble resisting power and lack of development. On the other hand, many conditions can be outgrown, as the structures and organs increase in size and strength develops.

Most deaths in the first year are due to marasmus, affections of the gastro-intestinal tract and to bronchopneumonia. Practically the only deaths due to nervous origin are from meningitis and convulsions. Of the acute contagious diseases, usually measles and pertussis are the only offenders. Of the chronic diseases, tuberculosis stands alone.

Sudden deaths occur from the following causes: malformations; internal hemorrhage; asphyxia from overlying; asphyxia from the aspiration of food into the larynx and trachea; asphyxia from enlarged thymus gland; atelectasis; convulsions; and marasmus.

In the second year there are the same causes of death as in the first, with the exception of marasmus, which fortunately does not extend into this period.

From the second to the fifth year scarlet fever, diphtheria, general diseases of the lungs, and tubercular meningitis are the diseases causing death.

From the fifth to the fifteenth year there is low mortality. It is chiefly made up of deaths resulting from diphtheria, scarlet fever, diseases of the lungs, tubercular meningitis, 
diseases of the bones, appendicitis, rheumatism, and cardiac conditions.

Quarantine.-The diseases which must be isolated by the regulations of the Board of Health of most cities are as follows: Scarlet fever, smallpox, diphtheria, and cerebrospinal meningitis.

Diseases in addition to the above which have to be reported are: Typhoid fever, chickenpox, measles, whooping-cough, tuberculosis, tetanus, erysipelas, hydrophobia, and the following uncommon diseases in childhood: Yellow fever, cholera, typhus, and relapsing fevers. 


\section{CHAPTER II \\ NURSING IN CHILDHOOD}

THE problems which confront the nurse in the management of children are vastly different from those encountered 'among adults, but the fundamental principles of nursing are the same. The methods of treatment are in many respects identical, or only slightly altered to adapt them to the young patient. The methods of amusement and entertainment can be appreciated by all who have come in contact with children, and success in handling them depends entirely upon the nurse's temperament.

It is the professional side of the nurse, her value to the physician in attendance, which demands the special training in children's diseases. It is necessary to know the symptoms and to be able to report them intelligently. This calls for careful, trained observation, as the child cannot describe its feelings accurately. The detail of symptoms cannot be recited by the patient, but has to be recognized and tabulated by the nurse.

It is necessary to understand the principles of infant feeding and hygiene. These are the two most important subjects of pediatrics. Knowledge of milk mixtures and their preparation is essential. Accuracy is demanded both by the infant's digestion and by the physician. The delicate mechanism of an infant's stomach cannot digest foods unless they contain the proper proportion of fat, sugar, and proteid. The physician's whole plan of treat- 
ment is rendered useless unless he knows the percentage of these ingredients in the mixture the child is getting. 'The proper methods of feeding in malformations and intubations must be understood.

Hygiene is a vast subject in itself. In infancy the surroundings and the personal attentions are of greater value than at any other time of life.

It is necessary to understand the significance of the stools in infancy. The character of these acts as a guide to the physician in the treatment of the case and in the construction of his milk mixtures. In the same way the character of the romit is important.

The methods of treatment in childhood must be thoroughly comprehended. The subject of prophylaxis and the care of the contagious cases is the field in which the nurse is in supreme command. By never permitting a lapse in her technic nor carelessness in her methods she does more for the prevention of disease and the health of the human race than any other factor in medicine.

\section{GENERAL HYGIENIC MANAGEMENT OF CHILDREN}

The Newly Born.-Immediately after birth the child should be wrapped in a blanket and placed in a warm room. The eyes should be washed with boric acid or, in cases where there has been a pre-existing vaginal discharge one or two drops of a 2 per cent. solution of silver nitrate should be used (Credé's method). The child should then be thoroughly greased and given a warm bath at a temperature of $100^{\circ} \mathrm{F}$. An antiseptic dressing should be applied to the cord and a flannel binder placed around the abdomen.

It is preferable to have the child sleep in a crib. 
Care of the Cord.-The cord should be kept dry and disturbed as little as possible until it drops off; this usually occurs on the fifth day. After this has happened an antiseptic dressing and a square pad should be placed over the navel and held firmly in place by the binder, to prevent umbilical hernia.

Bathing.-After the separation of the cord the full bath can be given daily. The water should be about $100^{\circ} \mathrm{F}$. The middle of the day and the warmest part of the room are the time and place to select. The bath should take only a few minutes, and vigorous rubbing should be avoided.

Clothing.-This should be light in texture, warm, and nonirritating. The chest and arms should be covered with a woolen undershirt, and all clothing should hang from the shoulders. Canton flannel or stockinet make the best diapers. The feet must be warm, as cold feet are responsible for many attacks of colic and indigestion (Holt).

The night clothing should consist of a light flannel gown, hung from the shoulders. Too much covering may cause disturbed sleep. In summer the outer clothing should be light and the underclothing of the thinnest flannel or gauze obtainable.

Special Hygienic Measures.-The eyes should be washed with boric acid for the first few days and at any time upon the appearance of a discharge from the eye. Infants should be kept in a darkened room. The temperature should be taken daily during the first week (see page 25).

The mouth should be cleansed with a soft rag wet with sterile water. A solution of bicarbonate of soda, $20 \mathrm{gr}$. to the ounce, should be employed if there are any signs 
of inflammation or thrush. In such cases the mouth should be cleansed with this solution after each feeding.

The breasts in both sexes often become swollen a few days after birth. If they are not interfered, with this disappears in a short time.

Genitals. - In boys the foreskin should be retracted daily and greased. The skin is very delicate in infants; the urine frequently causes scalding and blistering of the surfaces, especially in fat babies. If this occurs the napkins must be removed as soon as soiled; the skin should be bathed only once a day with water (for all other cleansing purposes olive oil should be used); and a powder consisting of balsam of peru, Io per cent. talcum, starch, cornmeal, or stearate of zinc, dusted upon the inflamed areas, in all the folds of the skin, and over the diaper. The best dusting powder is probably the balsam of peru, Io per cent., combined with stearate of zinc. Bran baths are advantageous at times (see page 407).

Airing.-In summer a newly born babe can be taken out at the end of the first week; in winter, at one month. All children should receive all the sunshine and fresh air obtainable.

Sleep.-During the first few weeks a child sleeps from twenty to twenty-two hours during the day. An infant should not be allowed to sleep at the breast or with a nipple of a feeding bottle in its mouth. The babe should be awakened every two hours for its feeding.

Infant feeding is discussed in Chaps. XVII. and XVIII.

Infancy. - Bathing. $-B y$ the sixth month the temperature of the bath can be reduced to $95^{\circ} \mathrm{F}$. and by the end of the first year to $90^{\circ} \mathrm{F}$. Older children should receive a cold douche with water of about $70^{\circ} \mathrm{F}$. after the bath, while standing in a tub of warm water. 
At times infants get blue after the bath, especially if delicate. Under such conditions it is better to discontinue tub bathing and depend upon the bed baths.

Clothing.-The abdominal band can be dispensed with after the first few months. In very thin infants it may be continued, to maintain the proper protection to the abdomen.

Low neck and short sleeves should not be allowed. The night clothing should be a light flannel gown hung from the shoulders. The night clothes should be an entirely different set from those worn during the day.

Special Hygienic Measures.-The teeth should be kept clean to prevent caries. The child should be trained to have its bowel movements and urinate at selected times.

Sleep.-During the first six months the child will sleep from sixteen to eighteen hours during the day. At one year it requires from fourteen to fifteen hours of sleep and at two years, thirteen or fourteen hours. An infant's position should be changed often during sleep.

Exercise.-An infant usually obtains plenty of exercise from crying and throwing its arms and legs about. Walking should be attempted during the period from the eighth to the sixteenth months, provided there is no tendency to rickets. Talking should be encouraged.

Childhood.-Bathing.-During childhood the warm bath should be given at night and the cold bath, or sponge in the morning.

Clothing.-Woolen undergarments in winter and light textures in summer are the rule. The night clothing should consist of woolen union suits with feet, if there is a tendency to get from under the covers.

General Hygienic Measures.-The bowel movements 3 
should be kept regular. Any illness or disorder should be immediately attended to.

Sleep.-At the age of four years eleven or twelve hours sleep are required.

Exercise.-The playroom should be cool-from $60^{\circ} \mathrm{F}$. to $65^{\circ} \mathrm{F}$. (Holt). The clothing should be loose, to give the freest possible motion of the muscles. Out-of-door exercises are the most healthful.

The proper methods of feeding for children over two years of age is discussed in Chapter XVIII.

Youth.-Sleep.-The amount of sleep required from the sixth to the tenth year is from ten to eleven hours. From the tenth to the fifteenth year at least nine hours of sleep are necessary.

An out-door life with plenty of exercise, frequent bathing, and instant attention to ailments lays the foundation for sturdy manhood and womanhood.

The nursery should be the sunniest and the best ventilated room in the house. Nothing which could contaminate the air of the room should be allowed. The temperature should be from $68^{\circ} \mathrm{F}$. to $70^{\circ} \mathrm{F}$; no higher. The room should always be thoroughly aired at night. The floors should be covered with rugs, as they are cleaner than carpets. An infant requires about 1000 cubic feet of air, older children about 700 to 800 cubic feet.

Premature Babies.- The conditions which have to be combated under these circumstances are the problems of maintaining the borly heat, and feeding.

Incubators. - These are so arranged that an even temperature may be maintained: $98^{\circ} \mathrm{F}$. in very delicate infants and from $85^{\circ} \mathrm{F}$. to $95^{\circ} \mathrm{F}$. in more robust babies. At the same time the air is moistened and ventilation is secured. 
In constructing an incubator the lower portion consists of a hot-water tank (hot-water bottles may be used), above which is an inlet for air. The bed should occupy a position midway in the air chamber and be so arranged as to allow the air to circulate freely around its foot. An exit for the air should exist above the child's head. A moistened sponge should be placed at the foot of the bed.

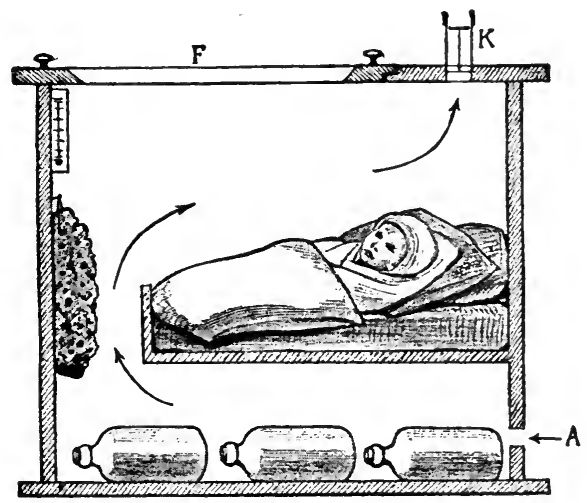

Fig. 10.-Cross-section of a wooden incubator of simple construction, with glass lid $(F)$ : The air enters through the opening $A$, which can be regulated by means of a damper, passes over the bottles filled with hot water, the saturated sponge, and the thermometer, and escapes through the ventilator $K$. (Frühwald and Westcott).

The child is kept in the incubator until it reaches full term. Before removing, the temperature should be gradually lowered.

In feeding these children, gavage often has to be resorted to. At seven months of age $\frac{1}{2}$ ounce should be given every hour and a half. At eight months $\frac{3}{4}$ ounce at the same intervals.

In small full-term babies it is also necessary to maintain the body heat. This is best accomplished by wrapping 
them in cotton and blankets. Hot-water bottles may also be employed.

\section{ROUTINE EXAMINATIONS}

Temperature.-When taking the temperature of the child it is not always possible to teach them to hold the

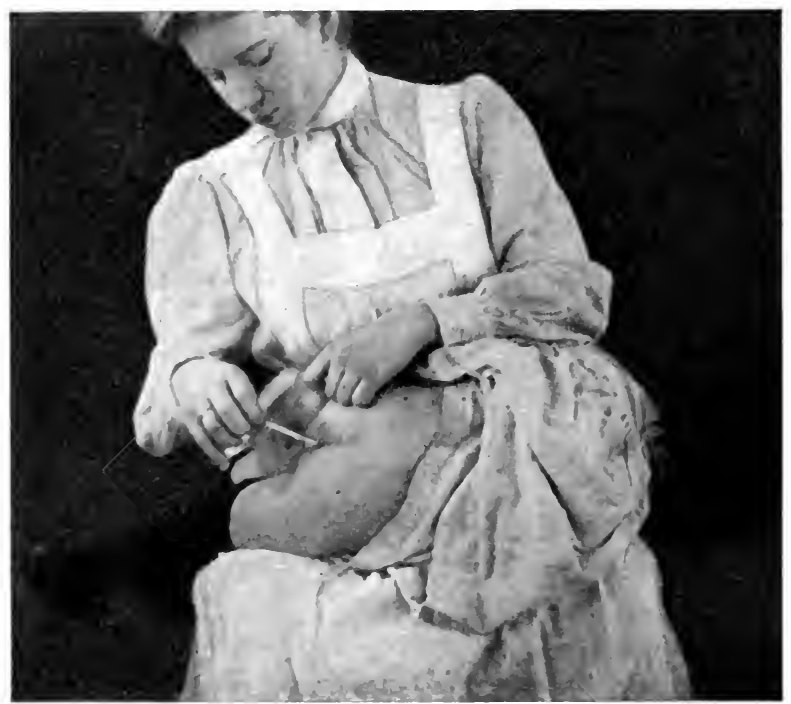

FIG. 11.- Methorl of taking the rectal temperature of an infant or young child (Kerr).

thermometer in the mouth properly, and for young children and infants this method is impracticable. The rectum, the axilla, or the groin are then utilized for this purpose. The thermometer should be greased with ordinary vaselin when introduced into the rectum and the temperature marked "rectal" on the chart or read one degree lower than the mercury indicates, as the local temperature in 
that part is about one degree higher than in the mouth. If an infant struggles while taking a rectal temperature, turn it on its face, or hold its face downward on your knee. When the thermometer is inserted, with the child in this position, it should be pointed downward, toward the umbilicus, as the axis of the rectum has been changed.

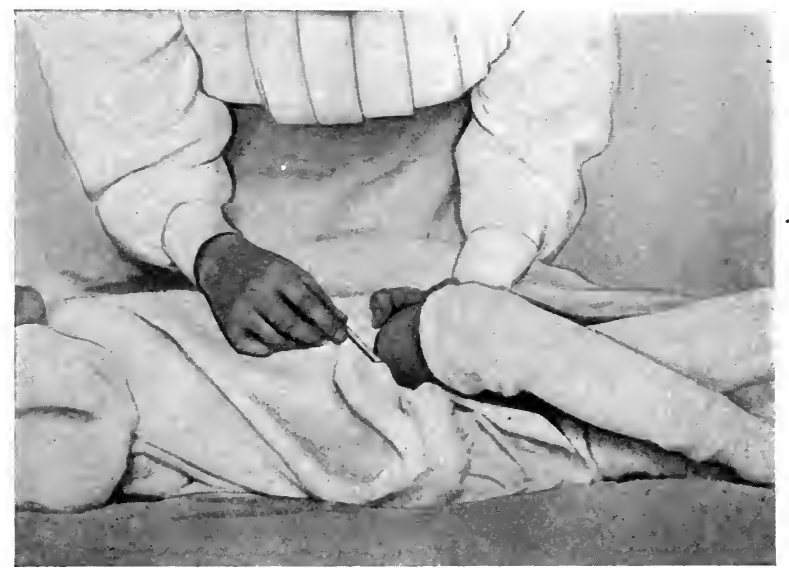

FiG. 12.- The method of obtaining the temperature at groin. The skin should be free from moisture. The thermometer is laid in the fold of skin between thigh and abdomen and is held in place by crossing thighs.

When the rectum is diseased it should not be chosen for the place to take a temperature.

In the axilla or groin the temperature should be marked "axillary" on the chart or read one degree higher, as there is that much difference in temperature between these localities and the mouth. The skin should be thoroughly dry and the thermometer closely surrounded by folds of skin.

Pulse.-The pulse is best obtained in the groin or at the temporal artery in infants. The normal rate for the different ages is given on page 25 . 
Respiration.-The normal rate for the different ages is given on page 25 .

\section{COLLECTIONS FOR CLINICAL EXAMINATIONS}

Method of Collecting Urine.-In males it is a good plan to place the penis in the neck of a bottle which lies between the thighs and is held in position by a square of adhesive plaster, the center of which is pierced, making a hole large enough to grip the neck of the bottle.

In females a small pan placed under the buttocks will answer, or a bottle can be arranged as described above. If these methods fail, catheterize.

The diapers are saved in cases where the stools are to be examined.

The blood is examined to determine the number of red and white blood-corpuscles, the percentage of hemoglobin, and for malarial organisms. For method, see page 176 .

The sputum is examined for tubercle and other bacilli, and should be expectorated into a sterile, wide-mouthed bottle.

In children under four years of age, the best method to obtain sputum is as follows: Have an applicator with sterile cotton about the tip. Grasp the tongue, pulling it as far forward as possible, and pass the applicator back to the pharynx, keeping as close to the tongue as possible. 'The irritation of the pharynx will excite coughing, and the sputum may be swabbed out.

The applicator may be placed by the child's bedside, and when the nurse notices a severe attack of coughing the child is picked up and the sputum obtained in the same manner. Another very easy method of obtaining sputum is to insert a large (1 2 -ounce) eye dropper into one end of a 
catheter, compress the bulb, and pass the catheter back to the pharynx, then allow the bulb to expand, this produces suction and draws the sputum into the catheter.

Cerebrospinal fluid is examined for various organisms. For method, see page 192 .

\begin{tabular}{|c|c|c|c|c|c|c|c|c|c|c|}
\hline DATE. & 峛 & 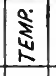 & 荇 & 它 & ș & $\begin{array}{l}\text { STOOLS } \\
\text { HARACTER }\end{array}$ & MEOTCINE & 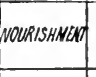 & 悹 & REMARKS \\
\hline $1 / 2 / 0 y$ & $3 A M$ & $1000^{\circ}$ & 108 & 24 & 1509 & softyellom & whisken st & $\begin{array}{l}\text { MilkgL.... } \\
6.5\end{array}$ & $3 \operatorname{hn}$ & \\
\hline " & GaM & $994^{\circ}$ & 96 & 24 & & & whistry y" & $\begin{array}{c}\text { Mithky,w } \\
6.9\end{array} \mid$ & $15 \mathrm{mmen}$ & Restless \\
\hline$"$ & gAM. & $1000^{\circ}$ & 108 & 26 & 605 & oftébrown & whiskry 站 & Milk $2 . \cdots$ & & Perspiring \\
\hline & LAMM & $103^{\circ}$ & 120. & 28 & & & whiskens & Mickiclu & $\operatorname{lin}$ & Vomited feeding \\
\hline$"$ & 3PM. & $1022^{\circ}$ & 116 . & 26 & 89 & & Whithey st & 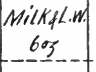 & & \\
\hline - & 6PM. & $102^{\circ}$ & 108 & 22 & & & Whothey y? & 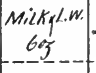 & $2 \mathrm{hn}$ & \\
\hline$"$ & QPM $\mid$ & & 108. & 2 & 25 & & Whickery" & 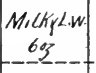 & & \\
\hline$"$ & RP,M. & $100^{\circ}$ & 108 & 22 & & & whitheng" & 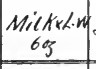 & $3 h r$ & \\
\hline & & $t=0$ & & & 415 & soft & Whistreys? & 489 & $9 / 1 / 4)$. & $\begin{array}{l}\text { Resting } \\
\text { comfortably }\end{array}$ \\
\hline
\end{tabular}

Fic. r3.-Method for keeping nurse's record.

Diphtheria bacilli are looked for in smears taken from the nose and throat. It is at times desirable to have the nurse obtain the culture from a suspected case of diphtheria. To do this she must have a platinum loop and a testtube containing the proper culture media (Löffler's blood- 
serum). These tubes should always be kept on ice until needed.

Before using the platinum loop it should be heated in the blue portion of an illuminating gas flame or, better, over a Bunsen burner until it is red hot.

After carefully removing the cotton stopper from the end of the test-tube, and holding it between the fingers in such a way that the portion which enters the tube comes in contact with nothing, the suspected spot in the throat or nose is gently rubbed with the loop of platinum. 'The thin film which forms between the loop is then spread over the culture media, being careful not to allow it to touch anything in transferring it from the infected area to the media. The cotton stopper is then reapplied, and the platinum wire again heated as before to destroy all germs it may still have upon it. The culture media should then be placed in a thermostat as soon as possible. Other discharges or parasites, like pus, pleural fluid, intestinal worms, etc., should be preserved in sterile testtubes.

\section{SPECIAL INSTRUCTIONS}

Never leave a child alone in a tub.

The infant should be weighed twice a week.

The personal care of sick children demands closer attention than that required in adults. Often the child cannot express his want at all. The study of the various cries, as described on page 26 , will often give the nurse an insight into their desires.

The fact that the urine, feces, and vomited material are frequently passed without any indication on the part of the child renders frequent examination, as to these 
conditions, necessary. Children do not expectorate until four years of age; before that time the sputum is swallowed.

In institutions the hair should be carefully scrutinized for pediculosis capitis.

At times water is greatly craved by children, and this fact is often overlooked. They should have small amounts between feedings, except in acute gastritis or after an operation, when cracked ice can be substituted.

In cases of illness infants can be held in the arms, excepting in cases which require absolute rest. Older children must be moved from side to side. Delirious children may require cuffs and strait-jackets (see pages 432 and 433).

When a child is being held for examination, do not press its arms down on the front of the chest.

When any portion of the respiratory tract is involved, do not tuck the bed-clothing in too tightly.

Sponging with tepid water (or a hot bath, $110^{\circ} \mathrm{F}$.) will often quiet a restless child. Observe children's spines; slight deformities, if recognized early, are easily corrected.

Children with rickets should be kept off their feet, and the nurse should train them to sit and lie straight. Children's toys should be boiled frequently, when possible, if there is a tendency to place them in the mouth. Infants' rattles and articles upon which they are permitted to bite should be tied in such a way that they will not fall upon the floor, and should also be frequently boiled.

Always place a clean sheet, preferably covered with a blanket or some similar device, under the infants if they are upon the floor. The nursery should be covered with a fabric which is easily cleaned and readily changed.

All draughts should be avoided when children are play- 
ing on the floor. A child should not be allowed to lie under an open window.

Children should be kept as quiet as possible after their evening meals, to avoid "night terrors."

Do not allow a child to lie or sit gazing at the sun.

When the rectum is diseased it should not be chosen as a place to take temperature.

When giving a rectal irrigation in any of the diseases of the lower bowel, it must be done slowly in order to avoid pain.

Cold compresses to the rectum or the introduction of small pellets of ice into the rectum will relieve straining or tenesmus.

Atomizers are used for sprays and nebulizers where oil is the base of the spray.

Before pouring boiling water into a hot-water bag put in a small quantity of cold water. This will prevent the seams from spreading.

For the proper care of milk in the house, see page 364 .

Remember that milk left uncovered for fifteen minutes may render all the care and asepsis practised at the dairies useless.

All "can openers" used to open tins of condensed milk and buttermilk should first be sterilized by boiling.

The same care must be taken of condensed milk and buttermilk as fresh cows' milk, as infection is just as readily carried by the one as the other (see page 365 ).

Before preparing milk mixtures and before feeding children, wash the hands thoroughly. If it is necessary to handle anything which might contaminate the hands while feeding, rewash them immediately.

After bottles have been sterilized they should not remain 
uncovered, but immediately stoppered with sterile cotton. When the bottles are being filled with milk, do not lay this sterile cotton down carelessly and then replace it. Keep it sterile.

All vessels in which milk has stood for any length of time must be thoroughly scalded before refilling.

Milk should not be kept warm in Thermos bottles or by any other method for any length of time. This applies even to pasteurized milk.

All nipples should be kept in a solution of boric acid when not in use.

To prevent nipples from collapsing, hold the bottle at such an angle that the nipple is always filled with milk. If for any reason a nipple is removed while the babe is nursing, do not put it down carelessly; it is better to drop it in a receptacle containing boric acid solution.

When diarrhea develops, stop the milk immediately.

A child should never be "jumped up and down" or rocked while it is being fed or immediately afterward.

If there is regurgitation immediately after feeding, sit the infant upright. Often there will be an eructation of gas which will eliminate this tendency.

Do not examine a child's throat immediately after feeding.

Hot-water bottles to a child's feet during bed baths, and after all baths, will prevent chills.

Keep children's feet covered at night; it prevents colic.

Sudden marked rises in temperature in children who have been well should direct your attention to the throat, and if no local manifestation of disease is present there, the ears should be examined. The ears should be examined when children are extremely restless at night and when they often cry out suddenly without apparent cause. 
Special attention should be paid to croup, particularly to attacks that do not occur at night; it may be laryngeal diphtheria.

In infectious diseases the nurse's sleeves should be rolled up above the elbow; in contagious diseases she should wear a cap and gown in addition.

Special attention to the ears and eyes is always necessary in measles. Disinfect the sputum and vomitus in cases of pertussis.

Keep children suffering from nephritis and rheumatism between blankets while in bed.

In vaginitis a pad should be worn.

When shaving the head of girls for a mastoid operation, leave a lock of hair in front which can be drawn over the scar. It can be retained in place under adhesive plaster at time of operation. If the physician gives his consent a shampoo before the operation makes the head feel more comfortable during the weeks the bandage must be worn.

When giving lavage and gavage be sure to have something between the teeth to prevent the child from biting the tube, for if it should be swallowed, it can only be recovered by opening the stomach.

In cases of shock, where nothing is at hand, a pint of hot coffee may be injected into the rectum.

Cradles may be improvised by turning chairs upside down.

If cotton cannot be obtained, newspapers may be substituted as a covering for poultices.

In the country or in houses where the proper facilities cannot be procured, a moist atmosphere can be maintained by placing hot bricks in a dish-pan half-filled with cold water. 
Whenever you are instructed to sterilize a thermometer, it should be boiled, but be sure it registers $212^{\circ} \mathrm{F}$., else it will break.

Never make a promise to a child unless it can be kept.

Prophylaxis and Nursing in Contagious Diseases.These are fully taken up under the various diseases.

Feeding.-Special cases are described under their proper headings. Chapters XVII and XVIII fully describe feeding during infancy and childhood.

Treatment is given when an intelligent understanding of the underlying reasons are necessary for proper application. As a rule, liquids should be employed. If pills or powders are ordered, the physician will usually instruct the nurse to crush or dissolve them before administration. Some drugs, like salol, however, are not soluble. In Chapter XIX the various therapeutic measures employed in childhood are described. Nursing of the various diseases is included in each chapter. 


\section{CHAPTER III}

\section{DISEASES OF THE RESPIRATORY TRACT}

THE respiratory tract consists of the nose (rhinos); the epiglottis; the larynx (glottis); the trachea; the bronchi (divided into the large, the medium, the small or capillary tubes); the air vesicles; the pleura (a serous membrane covering the lungs and lining the chest walls); and the mucous membrane lining all of the air-passages.

In disease of the respiratory tract one or more of the following symptoms is always present: (1) Cough; (2) expectoration; (3) dyspnea; (4) accelerated or diminished respirations. Pain and fever will be associated with these symptoms if inflammation is present. There will be an exudate if the inflammation involves a serous membrane and an abnormal secretion of mucus when a mucous membrane is attacked.

Cough is divided into dry, moist, and laryngeal. These are also spoken of as hard, loose, and croupy coughs.

Cough results from all inflammations of the respiratory tract; it is heard in certain of the acute infectious diseases with which are associated catarrhal conditions of the respiratory tract, such as whooping-cough (pertussis), measles (rubeola), and influenza; reflexly, it is caused by irritation of the mucous membrane lining the tract and by pressure on the recurrent laryngeal nerve.

Dry cough is a hard, tight cough and has no associated 
expectoration. It is seen in the first stages of acute bronchitis, in most chest diseases of early childhood, in pleurisy, and in reflex cough.

Moist cough is accompanied by copious expectoration. It is seen in the later stages of bronchitis, convalescent

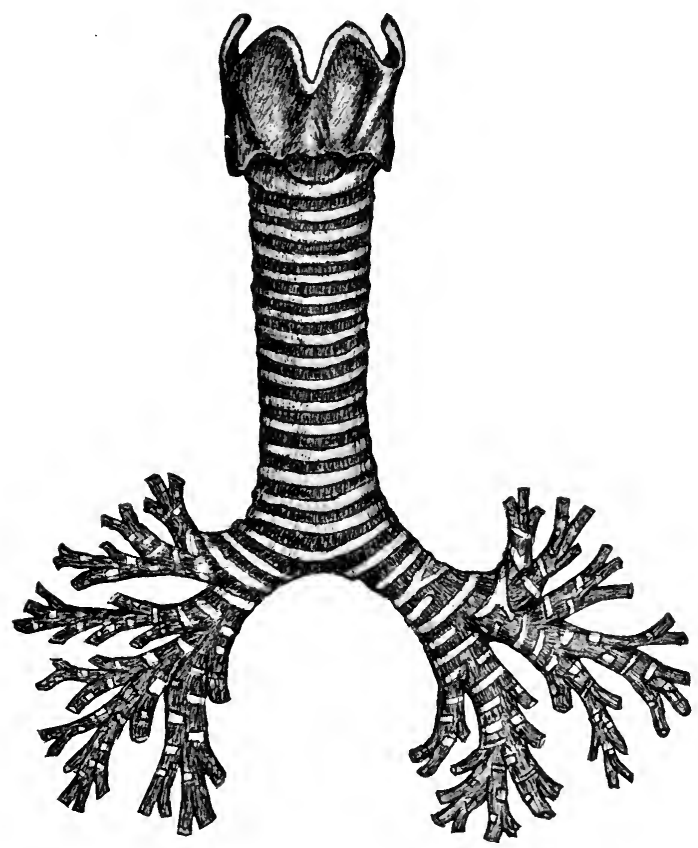

Fig. 14.-Larynx, trachea, and bronchi (Morrow).

pneumonia, and in most chronic diseases of the respiratory tract.

Laryngeal cough, also termed croupy cough, has a characteristic hard, barking intonation. It is heard especially in laryngitis, spasmodic croup, and whooping-cough. 
Expectoration results from the abnormal secretions of the mucous glands of the respiratory tract.

Children do not expectorate until after four years of age; previous to that time the sputum is swallowed.

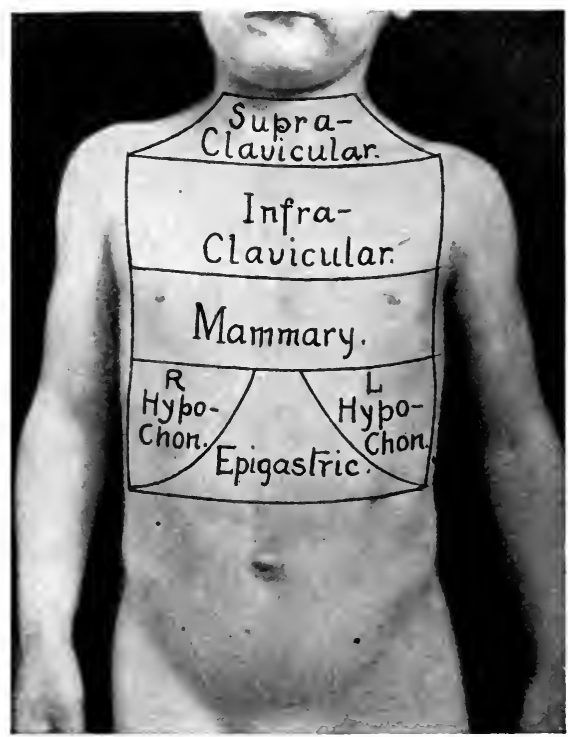

FIG. 15.-Topographic areas of the anterior thorax (Kerr).

The expectorated material may be mucus or mucus mixed with pus (mucopurulent). It may be the so-called rusty sputum, referring to its color, due to being tinged with altered blood. It is seen in advanced croupous pneumonia. Sputum containing fibrinous shreds is seen in diphtheria and in fibrinous bronchitis.

Dyspnea is difficult breathing, with or without increase in the number of respirations. It may be present on inspiration, expiration, or both. 
Dyspnea is caused by any obstruction to the free passage of air to or from the lungs. It is also caused by conditions which render the air capacity of the lungs less than normal, or which in any way interfere with the function of the lungs.

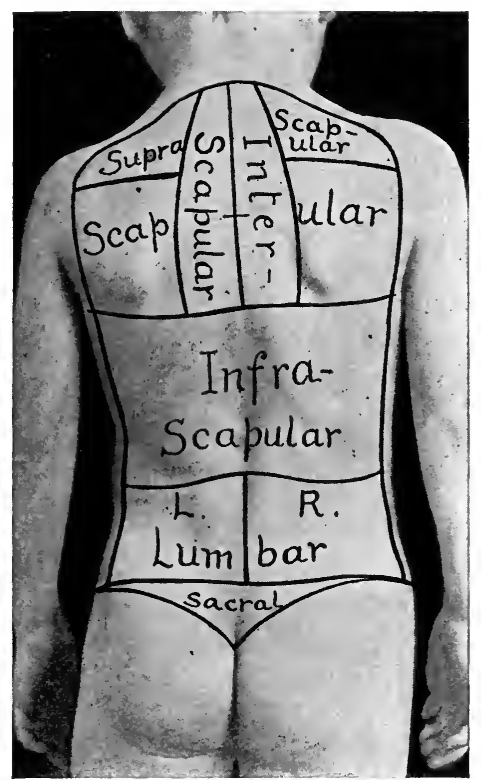

FIG. 16.-Showing the topographic areas of the back (Kerr).

Orthopnea is the name applied to the posture of a child who, on account of dyspnea, has to have the chest elevated so that it assumes a sitting or semireclining position in bed. It is usually seen in those suffering from heart disease.

Respiration.-The normal number of respirations 
per minute varies according to the age of the child. In the newborn they are from 30 to 50 per minute; in the first year from 28 to 30 per minute; at five years from 22 to 25 per minute; at 14 years about 20 per minute. The ratio between the respirations and the pulse-beats is one to four.

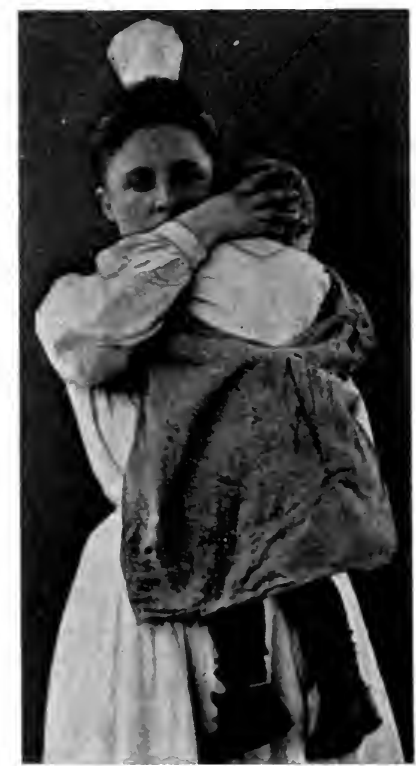

Fig. 17.-Position for examination of back of chest. The child can be perfectly controlled by the nurse in this position (Kerr).

Accelerated respirations are noted in fever and in all inflammatory diseases of the lungs.

Diminished respirations are seen in meningitis, in the spasmodic affections of the respiratory tract, and in uremia. 
Cheyne-Stokes respiration is the name given to a peculiar form of breathing seen in desperately ill children. It is characterized by a cycle. Following an interval, during

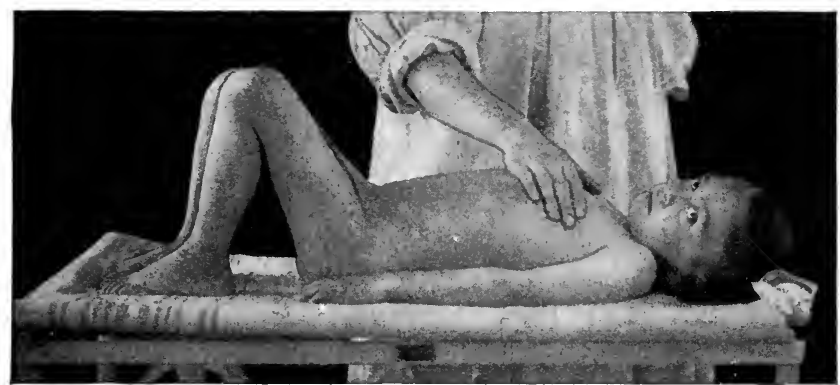

FIG. 18.-Counting the respirations (Kerr).

which there is no attempt on the part of the patient to breathe, the respirations begin slowly; they are deep and there is a short period intervening between each breath. Gradually they become more and more rapid, and at the

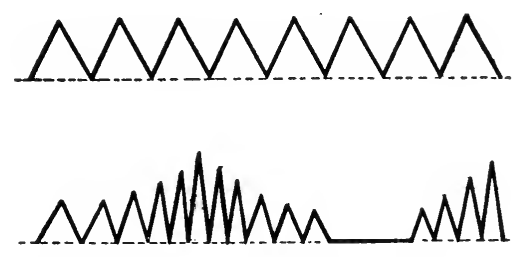

Fig. 19.-Diagram illustrative of normal (upper line) and Cheyne-Stokes respiration (lower line) (Kerr).

same time more shallow, until there is very rapid superficial breathing. They then gradually diminish in frequency and become deeper and deeper, the reversal of the former phenomena, until they again cease. The interval in which there is no attempt at respiration may 
last for thirty seconds. The cycle then begins again and goes on indefinitely, usually ending in death.

\section{RHINITIS}

Rhinitis means inflammation of the mucous membrane lining the nose. It is divided into acute rhinitis or coryza, and chronic rhinilis.

Acute rhinitis or coryza is the ordinary "cold in the head." It is due to an acute congestion of the mucous membrane lining the nose. The congestion causes swelling of the membrane to such an extent that the nasal chambers are closed and the patient is forced to breathe through the mouth. At first there is no secretion of mucus, the only apparent symptom being the general sensation of discomfort due to the occlusion of the nasal chambers. At the end of about twenty-four or fortyeight hours, however, there is a free discharge of mucus, very rapidly becoming mucopurulent in character. The discharge may cause excoriations of the nostrils and upper lip. In infants there is often a slight fever; in older children this is not often seen. At times the occlusion of the nose and the accompanying discharge is so great in infants that it is impossible for them to nurse, and the "dropper" has to be resorted to in feeding. Coryza may last from two days to two weeks, the discharge gradually becoming less and the nasal breathing more easy.

Treatment and Prophylaxis.-The tendency for some children to catch repeated colds is often due not so much to poor health as to faulty methods in their habits of living. Children who are kept within doors, in poorly ventilated rooms, and are not allowed to go out except in pleasant weather, and at such times are overloaded with 
clothing so that any exercise will cause them to perspire freely, are the victims of repeated colds. To overcome this tendency it is necessary only to enforce proper hygienic measures. A child should sleep in a room with the window open and be given a cold sponge in the morning. They should be allowed to play in the open air, except when inclement weather prevents. They should wear flannels covering their bodies and limbs all year; of light texture in summer and heavier in winter; and should have only the amount of clothing upon them which is seasonable.

As adenoid growths in the pharynx also predispose to repeated colds, they should be removed.

Abortive treatment for an acute coryza consists in giving the child hot drinks, such as lemonade, a laxative, and moderate doses of quinin and Dover's powder. From $\frac{1}{2}$ to I gr. of quinin and $2 \frac{1}{2} \mathrm{gr}$. of Dover's powder is the proper dose for a child of four years. After having been given Dover's powder the child must remain indoors for the subsequent twenty-four hours to avoid taking fresh cold.

Locally, inhalations of menthol or local applications of sweet oil will relieve the congestion somewhat. Antiseptic sprays, such as hydrogen peroxid and water, equal parts, or normal salt solution will be useful to alleviate the discharge when it is established.

Nasal syringing is described on pages $4^{\mathrm{II}}$ and $4^{\mathrm{I} 2}$.

Chronic rhinitis is due to a chronic inflammation of the mucous membrane lining the nose. It is divided into simple chronic catarrhal, hypertrophic, and atrophic rhinitis.

A chronic inflammation of the mucous membrane of 
the nose causes an increase in the secretions, so that there is a constant discharge, also an impairment of the sense of smell. This is easily understood when it is remembered that the terminal filaments of the olfactory nerve are in the highly sensitive mucous membrane lining the superior fossæ of the nose, and any permanent alteration in this membrane has a tendency to impair the function of smell.

Simple Chronic Rhinitis.-The principal symptom of this condition is a constant discharge, easily blown out of the nose. It is very often due to adenoid growths in the pharynx and is attended by excoriation of the nostrils, which is aggravated by the constant desire on the part of a child to pick its nose. This condition may last for years, the symptoms almost disappearing in summer and reappearing each winter. Epistaxis or nose-bleed is common.

Treatment and Prophylaxis.-The measures employed are hygienic in nature and consist in the child getting the proper amount of fresh air and exercise, in cold sponging, and in properly clothing the child. Adenoid growths should be removed.

Locally, antiseptic douching of the nose should be practised. Dobell's solution is one of the best remedies. When douching the nose in order to rid it of excessive secretions, the nostril should not be entirely closed by the nozzle of the syringe, leaving sufficient opening for the discharge to escape. The syringing must be carefully and gently done, so that the material is not forced along the Eustachian tube into the middle ear, which would give rise to an inflammation. The syringing should be done as described on pages $4 \mathrm{II}$ and $4 \mathrm{I} 2$. 
Hypertrophic Rhinitis. - In this variety the mucous membrane lining the nose becomes permanently thickened until the chambers are closed, thus forcing the patient to breathe through the mouth. This permanent thickening causes an impairment of the sense of smell and an overactivity of the follicles of the mucous membrane, which produces an increase in secretion. In addition there is a general catarrhal condition of the surrounding parts, with its attending symptoms, such as deafness from extension of the catarrhal inflammation along the Eustachian duct into the middle ear, watering of the eyes from occlusion of the lacrimal duct, and constant clearing of the throat from catarrh of the pharynx. This condition is infrequent in children.

Atrophic rhinitis is rarely seen in children under twelve years of age. It is the opposite from hypertrophic rhinitis, in that the mucous membrane, instead of becoming thickened, is found to be thinned and stretched out, and the nasal chambers, instead of being occluded, are enlarged. There is an atrophy of the tissues of the nose. In this condition there is a very offensive discharge and the patients are pale and anemic.

\section{EPISTAXIS}

Epistaxis or nose-bleed, while not often seen in infancy and early childhood, is quite common in older children. It may be due to some local condition of the nose, such as an excoriation of the septum. It must be remembered that nose-bleed is also a symptom of incipient typhoid, malaria, and measles, and that it is seen in nasal diphtheria and following a paroxysm of whooping-cough. More often, however, it is due to a spongy condition of the 
mucous membrane which is seen in children who do not get the proper amount of fresh air and are over-dressed when exercising. 'This relaxed condition of the mucous membrane makes them liable to frequent spontaneous nose-bleeds. 'The blood usually flows from one nostril drop by drop and lasts from ten to twenty minutes, the total amount lost being small.

Treatment.--Children subject to nose-bleed should get plenty of fresh air, be properly dressed, and receive cold sponging in the morning.

During an attack the child should sit upright in a chair, the clothing should be loosened about the neck, firm pressure made over the bridge of the nose by holding it between the fingers, and ice should be applied to the bridge of the nose and the back of the neck. Small pellets of ice may be introduced into the nostrils or held in the mouth. If this does not give results, plugging of the nostrils with absorbent cotton may be resorted to. Compound tincture of benzoin, or lemon-juice, diluted, may be introduced into the nose. No astringent powders should be used locally on account of their tendency to produce sncezing, thus starting the nosebleed afresh.

\section{LARYNGITIS}

Laryngitis is divided into acute catarrhal, chronic catarrhal, syphilitic, and tubercular.

Acute catarrhal laryngitis is an acute inflammation of the mucous membrane of the larynx. In this condition, as in any form of acute inflammation of a mucous membrane, there is congestion and swelling of the tissues. The vocal cords are involved, producing hoarseness, the characteristic laryngeal, croupy cough, which is worse 
at night, and often there is pain on swallowing. These symptoms last from three or four days to a week, with a strong tendency to relapse.

Chronic catarrhal laryngitis, as in all forms of chronic inflammation of a mucous membrane, is characterized by the permanent thickening of the laryngeal tissues due to an overgrowth of connective tissue. This produces a tickling in the throat, huskiness of the voice, expectoration of thick, ropy mucus, and fatigue and pain after moderate use of the voice.

Tubercular laryngitis is rare in childhood. It follows tuberculosis of the lung, but at times it is primary. It is manifested in the form of a sluggish ulcer causing deep destruction of the vocal cords and consequent loss of voice.

Syphilitic laryngitis is rare in childhood. It may result in hoarseness, loss of voice, and stenosis of the larynx.

Treatment.-In laryngitis, inhalation of steam medicated with compound tincture of benzoin is always efficacious. In severe cases a croup tent may be used (see page 4I3). Cold compresses applied to the throat often control the cough.

\section{SPASMODIC CROUP}

This is the ordinary croup of childhood. It is caused by a spasm of the vocal cords which is excited by a congestion or a catarrhal inflammation of the mucous membrane of the larynx.

The most frequent exciting causes are exposure to cold and damp weather, indigestion, and constipation. Anything which may cause a mild catarrhal inflammation of the mucous membrane of the larynx can be the exciting cause of croup, for it is this congestion or inflammation 
which produces the spasmodic condition of the muscles of the larynx. The adductor muscles are the ones most involved.

Symptoms.-The attack occurs in young children between the ages of six months and five years. The child may have a little hoarseness and coryza during the day, and in the evening a laryngeal cough, hard and barking in character. The true attack of croup does not occur until several hours after the child has gone to sleep. It is awakened by severe paroxysms of coughing and by difficulty in getting its breath due to the closure of the larynx by the spasm of the vocal cords. In a severe case there is a marked dyspnea, the breathing is slow and labored, and the inspiration noisy, owing to the air whistling through the narrow opening between the cords. This stridulous breathing, as it is called, may be loud enough to be heard in the next room. All the symptoms of profound dyspnea are present: the nostrils dilate with each inspiration, the face is anxious, drawn, and beaded with perspiration, the lips and the tips of the fingers are slightly blue. The notches above the sternum and the clavicles are deeply depressed and the base of the chest retracted with each inspiration. All the muscles of respiration are brought into play and are very prominent from the effort to fill the lungs with air. The pulse is rapid, I 10 to I 20 beats per minute, and the skin is hot. Associated with these symptoms is the hard, metallic, croupy cough which at times may almost be incessant. The attacks last from a half hour to three hours. The cough gradually subsides, the breathing becomes less difficult, the child breaks into a free perspiration and falls asleep. The attack may recur on the same night. 
The next day, with the exception of slight hoarseness, the child seems perfectly well. The following night, however, there is often another attack of croup similar to the first and equally as severe, unless proper medical treatment has been instituted. The third night, also, may be attended with an attack which is not apt to be as severe as the preceding seizures.

Treatment and Prophylaxis.-As the attacks are exciled by mild catarrhal inflammation of the larynx, such defects as may predispose to this condition should be remedied. Hypertrophied tonsils and adenoids should be removed. Children should have plenty of fresh air and cold sponging. Constipation and indigestion should be avoided.

Treatment of the Attack.-A sponge moistened with hot water may be applied to the throat or the child may be put in a hot bath or a mustard tub ( $110^{\circ} \mathrm{F}$.). If these simple remedies fail, an emetic will often bring relief, the best being the wine of ipecac administered in dram doses until effective, or a little powdered alum mixcd with honey or molasses given in teaspoonful doses. In severe cases it may be necessary to resort to the inhalation of chloroform. The object of this plan of treatment is to relieve the spasm of the vocal cords.

A croup tent is a valuable adjunct to this plan of treatment (see page $4 \mathrm{I}_{3}$ ), the moist atmosphere soothing the inflamed mucous membrane and thus shortening the attack; it also diminishes the chances of recurrence. The steam may be medicated with compound tincture of benzoin, which increases the efficiency of this plan of treatment. 


\section{LARYNGISMUS STRIDULUS}

Laryngismus stridulus, also called child crowing, is purely of nervous origin (a neurosis), and does not depend upon a catarrhal condition of the larynx, like spasmodic croup. It is due to a complete spasmodic closure of the larynx, making it impossible, for a time, for the child to breathe at all. The spasm then relaxes and the air is drawn through the contracted larynx with a shrill, crowing sound. It is seen in children of a rachitic tendency between the ages of six and eighteen months and seems to be more common in males than females.

The attacks are frequently seen in children who have been closely confined in warm, stuffy rooms, and are often associated with enlarged tonsils and adenoids. The attack may be excited by a sudden draught of cold air, or, reflexly, from teething and gastro-intestinal disorders.

Symptoms. - The child may have a few mild attacks during the day or extending over a period of several days. This condition is very often confounded with whoopingcough, the "crowing" of the mild attacks closely simulating the whoop of pertussis.

When the attack is fully developed the child is awakened from sleep by a sudden arrest of the breathing and a tonic spasm of the muscles. (Tonic spasms are continuous spasms in which the patient remains rigid until the spasm relaxes.) The face is at first pale and later bluish, the neck rigid, the eyes rolled up, the body arched, the thumbs turned into the palms of the hands, the legs extended, and there is a complete absence of breathing. In about fifteen or twenty seconds the spasm relaxes and the air is drawn through the larynx with a shrill, crowing sound. At times the spasm is longer, and in a few instances asphyxia 
has resulted before it has relaxed. Several such attacks may occur on the same night, and, gradually decreasing in severity, they may extend over a period of one to two weeks unless proper medicinal measures are instituted.

To distinguish this affection from spasmodic croup remember that in laryngismus stridulus there is no croupy cough, hoarseness, or fever, but there is present a tonic spasm and the peculiar crowing sound.

Treatment and Prophylaxis.-Fresh air and cold sponging unless the shock of the sponging frightens the child into an attack; the rachitis should be treated; hypertrophied tonsils and adenoids should be removed, and gastro-intestinal disorders corrected.

For the attack the best treatment is to dash cold water on the face and neck, in an attempt to break the spasm. Mustard tubs $\left(110^{\circ} \mathrm{F}\right.$.) may be resorted to, and inhalations of chloroform in severe cases. At times it is necessary to perform intubation.

\section{EDEMA OF THE GLOTTIS}

This is a rare condition in childhood. It is dropsical in character, due to a serous infiltration into the submucous tissues of the larynx. It occurs sometimes in the course of scarlet fever, diphtheria, and facial erysipelas. It may occur abruptly in the course of Pright's disease.

Symptoms are those of suffocation due to the swelling of the tissues lining the larynx and to the consequent closing of the passage. It demands the immediate attention of a skilled physician, intubation being necessary in many cases. 


\section{BRONCHITIS}

This is an inflammation of the mucous membrane lining the bronchial tubes.

The three main divisions are acute bronchitis, chronic bronchitis, and fibrinous bronchitis.

Acute bronchitis presents such a difference in the grade of severity with which it attacks children that it is necessary further to subdivide it for comprehensive study. In children under two years of age bronchitis is always serious, hence we divide it into bronchitis of the very young, two years and under, and bronchitis of children over two years of age.

The reason that bronchitis is such a severe affection in the very young is on account of its tendency to extend into the smaller bronchi and verge upon pneumonia.

The smaller the caliber of the tube affected, the more severe the attack of bronchitis. Therefore, it is also subdivided into bronchitis of the large tubes, bronchitis of the medium-sized tubes, and capillary bronchitis, or, as it is usually termed, bronchopneumonia.

Symptoms in the mild form, or bronchitis of the larger tubes in a child under two years of age. Here the respirations will average about forty or fifty to the minute. The temperature ranges from $100^{\circ} \mathrm{F}$. to $102^{\circ} \mathrm{F}$. There is cough, hard and tight in character at first, but rapidly becoming loose. While the cough may be loose in character there is no expectoration. Children rarely expectorate before four years of age; previously the mucus is swallowed; sometimes this causes vomiting. There is some dyspnea and often a co-existing catarrhal condition of the nose and throat.

An attack of bronchitis lasts about a week. In children 
subject to colds, who develop a bronchitis during the winter months, the attacks are liable to recur until warm weather.

In the severe form, or bronchitis of the medium-sized tubes in a child under two years of age, the symptoms can hardly be distinguished from pneumonia. The onset, however, is not so abrupt, and the temperature does not

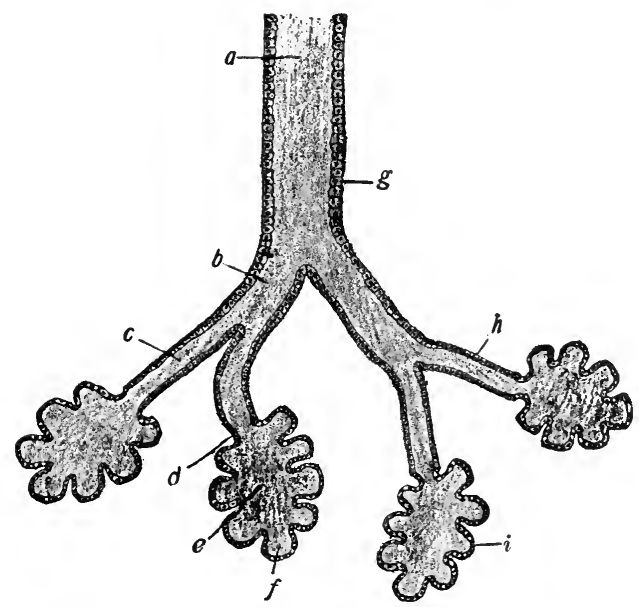

Fig. 20.-Diagram of bronchial terminations: $a$, Smaller bronchus; $b$, terminal bronchus; $c$, alveolar duct; $d$, constriction, or neck; $e$, space of infundibulum outlined by dotted lines; $f$, infundibula ; $g$, ciliated columnar cells lining small bronchus; $h$, nonciliated columnar cells lining alveolar ducts; $i$, flat epithelium lining alveoli (Leroy).

remain high so long. The attacks last three or four days and are accompanied by constitutional symptoms, such as prostration, apathy, and loss in weight. In this form of bronchitis there is always danger of an extension of the inflammation to the capillary tubes.

Capillary bronchitis is bronchopneumonia, and will be considered under that title. 
In children over two years of age bronchitis is not so severe an affection, although here we find the same conditions existing as in younger children; namely, a mild form due to an inflammation of the larger tubes and a severe form due to an inflammation of the smaller tubes.

Symptoms of acute bronchitis in children over two years of age consist in chilliness, malaise, some fever, cough, and dyspnea. The cough is worse at night; at first it is dry and painful, but later becomes loose and is accompanied by free expectoration of mucopurulent sputum. Children often complain of soreness and pain in the chest during an attack of bronchitis, which is aggravated by coughing.

Treatment.-Abortive.-A case of incipient bronchitis can be aborted by hot foot-baths, the application to the chest of a turpentine stupe or a mustard plaster to relieve the congestion of the bronchial mucous membrane, and the internal administration of hot drinks, quinin, and Dover's powder, from $\frac{1}{2}$ to I gr. of quinin and $2 \frac{1}{2}$ gr. of Dover's powder being the proper dose for a child of four years. When Dover's powder is administered the child should remain indoors for the subsequent twenty-four hours to avoid taking fresh cold. A laxative is always a safe adjunct to this plan of treatment. In younger children mustard paste applied to the chest for ten minutes and covered with a towel will often abort a forming cold.

Treatment during the course of the attack is aided by having the child live in a moist atmosphere. This can be accomplished by steam generated by a special apparatus or by the heating of a pan of water over a small gas stove in the room. Compound tincture of benzoin added to the water increases the efficiency of this method of treatment. An oiled-silk or cotton-batting jacket, as an 
adjunct to the treatment of bronchitis in children, is at times employed.

In severe cases of bronchitis children sometimes have an attack of suffocation and respiratory failure. The indications here are to remove any obstructing mucus, to compel the child to take deep respirations, and to get as much blood to the surface and into the extremities as possible, in order to relieve the overloaded right heart. Inverting the child will often cause the mucus to run from the mouth, and a mustard tub ( $110^{\circ} \mathrm{F}$.) will accomplish the rest. Oxygen and heart stimulants must be given in these emergencies.

In the milder forms of bronchitis the children should be confined to the house, but not necessarily to bed. In the more severe attacks it is better to put the child to bed, and in the very severe cases it should be placed in a croup tent.

Nursing.-The sleeping-room of the infant must not be too cold, never below $60^{\circ} \mathrm{F}$. It should be well ventilated, receiving a thorough airing two or three times a day. An open fire is a good adjunct.

Hot baths, at a temperature of $110^{\circ}$ to $115^{\circ} \mathrm{F}$., are frequently ordered.

The clothing should be warm, the night-dress of flannel, and the feet should be protected against cold. The bedcovers should never be tucked in too tightly.

The mouth and nose should be kept clean with antiseptic sprays and douches, all mucus should be cleared from the throat, and the chest thoroughly rubbed with camphorated oil or a similar counterirritant. At times the chest may be enveloped in a cotton or oiled-silk jacket.

A few drops of glycerin undiluted or a small lump of 
sugar will frequently control a severe attack of coughing. .

Cold compresses applied to the throat will also give relief.

The child should be allowed to sleep as much as it will, but should be awakened for its food and medicine at regular intervals. It is always best, when possible, to combine these two periods, to avoid unnecessary disturbance.

Infants can be held in the arms and older children can be about the room, unless fever is present.

The temperature, pulse, and respirations should be taken twice a day, unless the temperature is over $\operatorname{ror}^{\circ} \mathrm{F}$., when it should be taken every three hours.

Chronic bronchitis is caused by repeated attacks of acute bronchitis following one another so closely that the mucous membrane does not become entirely normal between attacks, each one leaving something behind. This causes the mucous membrane to become thickened by an overgrowth of connective tissue, as in all chronic inflammations.

Symptoms. - A chronic cough which is worse at night and expectoration which is most profuse upon arising in the morning are the main symptoms. The children are pale and thin, but do not become emaciated.

Fibrinous bronchitis is a rare disease in childhood. It is sometimes seen associated with diphtheria, when it is due to an extension of the membrane into the bronchi. At other times it is unassociated with that disease and is due to an inflammation of the bronchial mucous membrane and characterized by the formation of a false membrane. This membrane is dislodged by coughing and expectorated as little whitish balls, which, when unrolled under water, 


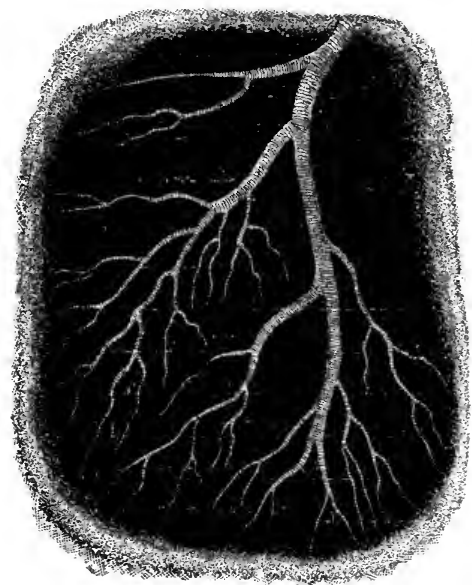

FIG. 21.-Large bronchial coagulum; chronic fibrinous bronchitis (Vierordt).

present the mold of the branching and ramification of the bronchial tubes affected. Acute and chronic forms are recognized.
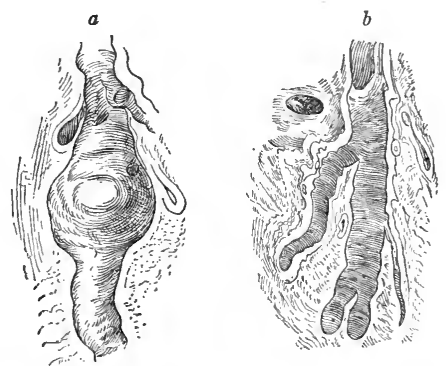

F1G. 22.-Bronchiectasis: $a$, saccular; $b$, cylindric; one-half natural size (Orth).

\section{BRONCHIECTASIS}

- Bronchiectasis is a dilatation of a bronchial tube. The whole tube may be involved or only a small portion may 
be affected. It is caused by a weakening of the walls of the bronchi from inflammation. While in this weakened condition cough causes the stretching and dilatation. It may produce a cylindric or a sacculated enlargement of the tube.

\section{ASTHMA}

Asthma is a paroxysmal dyspnea, due to a spasm of the bronchial tubes or to a swelling of the mucous membrane lining them. In children it is associated with marked catarrhal symptoms.

In young children true asthmatic attacks are not often seen, the condition resembles a severe bronchitis with an asthmatic tendency; that is, there are catarrhal symptoms present, with difficult, wheezing expiration. Such an illness, may persist for three or four weeks.

Attacks resembling asthma in adults do occur in childhood, usually in older children.

Symptoms.- In those subject to asthma the attack may be excited by a cold draught, the inhalation of dust, and by an overloaded stomach. The paroxysms appear suddenly and especially at night. There is such intense dyspnea that the patients have to sit upright with their arms in such a position as to bring into play all the muscles used in respiration. The respirations are not necessarily fast, but they are labored, and there is a loud, noisy, wheezing expiration. Cough is often present. Several attacks may occur in the course of a few days or they may be a month or more apart.

Treatment.-During the attack prompt relief often follows the inhalation of a few drops of chloroform or amyl nitrite.

Asthmatic attacks occurring in the course of cardiac 
or renal disease are named cardiac asthma and renal asthma, respectively.

Hay Asthma or Hay Fever. - In this condition, besides the asthmatic attacks, there is a coryza and a catarrhal condition of the nose and throat.

Hay fever is seen in the Spring and in the Fall. When it begins in May or June it is spoken of as rose cold. This lasts until the end of July. The time when hay fever is most prevalent is in the Fall. It makes its appearance in August and continues until the first frost. A peculiarity about hay asthma is that it is excited by the pollen of plants.

\section{PULMONARY EMPHYSEMA}

Abnormal distention of the lungs with air may result from two causes. The first to be considered is known as compensatory emphysema.

Compensatory emphysema is not a diseased condition of the lung affected; it is an extra expansion of the air-cells to accommodate more air. There is a certain amount of work to be done by the lungs at all times. Although a number of air-cells may be disabled from some cause, such as consolidation from pneumonia or pressure from a pleural effusion, the amount of work does not diminish; therefore, it is necessary for the healthy air-cells to distend and perform not only their own task, but also that which should be done by the diseased area. Consequently, these cells have a greater capacity for air than they did when all the lung structures were performing their normal functions. If the disablement persist, the compensatory enlargement is permanent.

Pathologic Emphysema.-The other cause is purely a pathologic condition in which we have a permanent 
distention of the air vesicles from stretching and thinning of their walls.

Causes.-These include cough, asthma, whoopingcough, laryngismus stridulus, and bronchitis. In such conditions there is some resistance to the free exit of air from the lungs and it requires a certain amount of extra effort on the part of the lungs to force the air out. This extra exertion falls upon the walls of the air vesicles and the strain causes them to stretch. Any condition where the walls of the air vesicles have to stand the strain of forcing the air out of the trachea is likely to cause emphysema.

Pertussis is the greatest cause of emphysema in childhood; the condition usually disappears, however, with the paroxysms of cough.

Symptoms.-There is great shortness of breath on account of the damage to the walls of the air vesicles. The distention and the loss of resiliency makes the effort to empty the lungs of inspired air very difficult, renders expansion impossible, and the chest immobile. The typical barrel-shaped chest seen in adults is not common in childhood.

Compensatory emphysema does not produce symptoms.

Emphysema occurs in young children on account of their undereloped condition and because the lung tissues are not strong enough to withstand sudden and violent strains.

\section{HEMOPTYSIS}

Hemoptysis is the name applied to the spitting of blood. The blood is ejected by coughing, is bright red in color, and frothy; it is mixed with sputum, and the subsequent expectorations are tinged with blood. 


\section{PULMONARY EDEMA}

Edema of the lungs is an effusion of serous fluid into the air vesicles and into the interstitial tissue of the lung.

Pulmonary edema is a common cause of death in many acute and chronic diseases which end by heart failure and the accumulation of blood in the lungs. At the termination of a disease which ends by heart failure the heart-beats gradually become weaker and weaker, the blood-current becomes slower and slower, and as the pressure within the arteries becomes less from the failing force of the heart, there is a leaking of the blood-serum through the walls of the blood-vessels into the air-cells which they surround, or into the tissue of the lung itself. This gradually continues until the accumulation of fluid practically fills the entire lungs, and death results.

Treatment.-If this condition should arise in the course of an acute illness such as pneumonia, the physician will probably order heart stimulants to be given immediately, especially strychnin, $\frac{1}{60} \mathrm{gr}$., digitalis, 3 to $5 \mathrm{~min}$ ims, and nitroglycerin, $\frac{1}{2} \frac{1}{50}$ gr., hypodermically, the doses being for a child of four years. In addition some form of counterirritation should be applied to the chest at the same time, in order to draw as much blood to the surface as possible. The best counterirritant is dry cups or a mustard plaster.

\section{ATELECTASIS}

The absence of air from a portion of the lung due to collapse of the air vesicles.

It may be congenital-due to deficient respiration. Children of low vitality, and especially premature babies, do not expand their lungs sufficiently, owing to feeble efforts at breathing. The air vesicles which are not fully 
expanded by the inspired air collapse, preventing any air from gaining access to such areas.

In order to overcome any tendency in this direction it is necessary to see that young children expand the whole of their lungs. The best way to accomplish this is to have the baby cry with vigor once or twice a day, and not allow it to remain perpetually in its crib. A certain amount of handling daily is necessary and overcomes the tendency toward shallow respiration.

Atelectasis may be acquired by the occlusion of a bronchus from some cause or other, or it may result from the pressure of a tumor or pleural effusion compressing portions of the lung.

\section{PLEURISY}

This is an inflammation of the serous membrane covering the lungs.

In children pleurisy may be $d r y$ or there may be an effusion. The effusion consists of a collection of fluid in the pleural cavity which is poured out by the inflamed serous membrane. It is composed of serous fluid and flakes of lymph. Such a collection of fluid is termed a serous effusion. This effusion may be purulent in character, when it is termed an empyema.

Serous effusions are less common in children than in adults; under three years of age this form is not seen. Empyema, however, is much more frequent in childhood than in adult life.

Pleurisy may involve one side of the chest, when it is termed unilateral pleurisy, or it may attack both sides of the chest, bilateral pleurisy.

A sacculated pleurisy is the term applied to a liquid effusion in the pleural cavity which is circumscribed and 
confined to pockets formed by adhesions. In all pleurisies there is a deposit on the membrane of a butter-like exudate of fibrin; this may form adhesions (adhesive pleurisy). These adhesions subdivide the pleural cavity into small pockets or they may completely separate one portion of the cavity from another by extending across from the costal to the pulmonic surfaces.

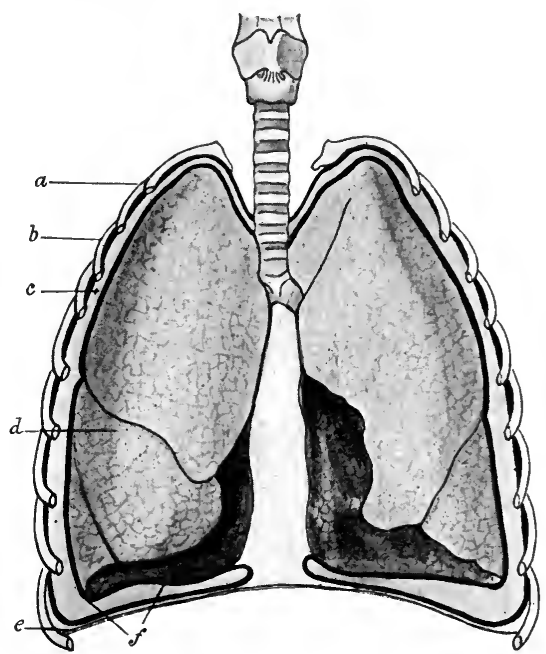

FIG. 23.-Diagram of pleural cavities : $a$, Ribs; $b$, costal pleura ; $c$, pleural cavities; $d$, lungs; $e$, diaphragm; $f$, pulmonic pleura.

Chronic pleurisy is an effusion of any nature remaining unabsorbed.

Causes.-Pleurisy in infants is probably caused only by extension of the inflammation from the lungs. Throughout childhood the most frequent cause is pneumonia. In nearly every case of consolidation in the lung there will be an inflammation of the pleura over such an area. 
Pleurisy is also seen in connection with tuberculosis. It is secondary to scarlet fever, typhoid fever, measles, and influenza, occasionally. In older children it may be due to rheumatism, and at times, though rarely, to cold and exposure.

Pleurisy is most often seen between the ages of one and five. It is more common in boys than in girls.

Pathology.- In the first stages of the attack the membrane becomes congested and is covered with a thin film of lymph. If the process now ceases, it is termed a $d r y$ pleurisy. This is the usual type in children under seven years of age. If the inflammation continues, an effusion will form, composed of straw-colored serum with flakes of lymph floating through it. The quantity may range from a few ounces to several pints, which in favorable cases is gradually absorbed. In large effusions the organs are displaced and the lungs compressed.

Symptoms.-Dry pleurisy.-The principal symptoms are pain in the side, increased by inspiration, and a dry cough, partially suppressed on account of pain. The patient lies on the affected side to keep it as quiet as possible, and also to allow the other side to fully expand. There is some fever, ranging from $102^{\circ} \mathrm{F}$. to $103^{\circ} \mathrm{F}$. The symptoms continue until the inflammation subsides. Adhesions may form and cause some retraction of the affected side.

Serous Pleurisy.-The symptoms resemble pleurisy in the adult. At first there is a typical picture of a dry pleurisy, with its accompanying symptoms. To this is added, on the third or fourth day, the signs of an effusion consisting of bulging of the interspaces of the chest, the relief from pain as the inflamed surfaces are separated by 
the accumulation of the fluid, and the appearance of cyanosis and dyspnea. The ordinary effusion is absorbed in a week or ten days, but at times it persists from delayed resolution for a much longer period.

Treatment.-This is the same in dry and serous pleurisy. It consists in the application of counterirritants and hot poultices for the pain. Sometimes strapping the side with strips of adhesive plaster will give relief. The physician will order heart and respiratory stimulants, such as strychnin, atropin, digitalis, and nitroglycerin, to be given hypodermically in case of emergency. At times it is necessary to aspirate the collection of fluid.

Nursing.-The room should be kept at an even temperature of $68^{\circ} \mathrm{F}$. and be well ventilated. Bathing should be restricted to sponging. The clothing should be flannel and protection of the feet is necessary. The bed covers should never be tucked in too tightly.

Sleep is often disturbed by pain, so that some counterirritant may be required to give relief.

Feeding should be at regular intervals.

The patient may be allowed to sit up after the fever subsides, but in large effusions exertion must be avoided, as sudden death has taken place under such conditions.

The temperature, pulse, and respirations should be taken every three hours as long as there is fever; later, twice a day is sufficient.

As primary cases of pleurisy are often tubercular, extra care must be taken with this form of the disease.

If the child's chest is tapped, a specimen of the fluid should be collected in a sterile test-tube with an aseptic cotton stopper. Disinfect the remainder before disposing of it.

Empyema or purulent pleurisy is common in children. 
It is most often a sequel of pleuropneumonia. After seven years of age it sometimes occurs in connection with tuberculosis. It also is a complication of scarlet fever, measles, and any of the acute infectious diseases.

Pathology.-In empyema the pleural cavity is filled with thick, non-offensive, greenish-yellow pus, amounting from a half pint to two pints. The left side is more commonly affected than the right, and at times it is bilateral.

Symptoms.-Following one of the acute infectious diseases there is an effusion found in the pleural cavity which does not show signs of absorbing. Before seven years of age any pleural effusion must be looked upon as the result of empyema, so rarely is serous pleurisy seen before that time.

The child becomes pale and thin, the respirations become accelerated, there is fever, but often not of the hectic type, as is usually seen when pus is present. hectic temperature is marked by a very irregular fever, alternating high and low, and is accompanied by sweats and chills.) The fluid gradually accumulates and dyspnea develops. If left to itself, the purulent effusion may kill by sepsis or it may perforate into the lung, the pus then being coughed up and expectorated. It sometimes perforates into the surrounding organs or tissues, causing a local abscess.

Diagnosis.-If there is any doubt as to the character of the fluid in the pleural cavity, this may be cleared up by puncturing the cavity with an exfloring needle; an ordinary hypodermic syringe is used for this purpose. At the point selected by the physician for puncturing, after the skin has been rendered aseptic by scrubbing with tincture of 


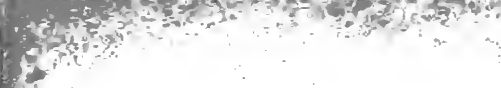

(n) 


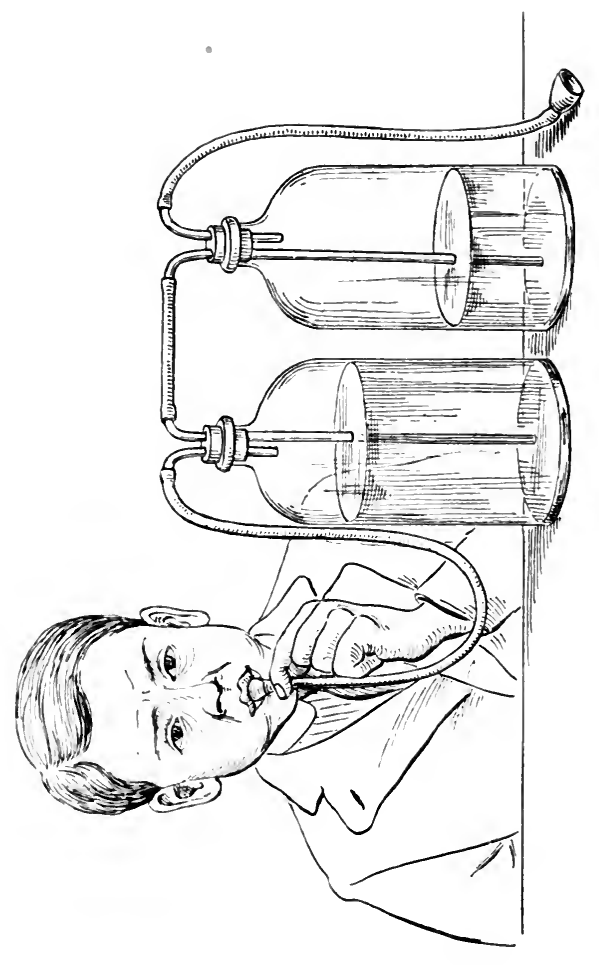

异总

$\because \underset{\Xi}{ }$

咅气

$\stackrel{0}{\circ}$

$5 \cong \stackrel{\Xi}{0}$

เo $0 \frac{\pi}{2}$

ఏ方

导亡 :

吾焉

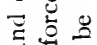

它念

롤

严造

○

芯芯

文先

ฮ 은

光 站

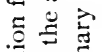

芯芯芯

ธิ ธิ

莺芯

芯总

$\Rightarrow=0$

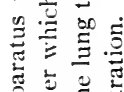

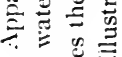

㤩总 
green soap and alcohol, a sterile needle attached to the syringe is plunged through an interspace into the pleural cavity and the syringe is slowly filled. The character of the effusion will at once be apparent, pus showing as a creamy-white fluid. At times the needle may not find pus, even when it is present. This may be on account of large flakes of lymph obstructing the needle or because the needle has penetrated too far and gone completely through the cavity. Care and repeated punctures will usually overcome this contingency.

Treatment.-After it has been definitely decided that the case is one of empyema there are two procedures which the physician may follow. In the large number of cases he will have the child operated upon. In a fewer number of cases he will have the pleural cavity aspirated.

Operation.-This consists in opening the pleural cavity, removing the pus, and draining. There are several methods employed. A simple incision or an incision and the removal of a portion of one or more ribs for better drainage are the methods usually followed. A rubber tube is inserted into the cavity to insure perfect drainage.

Aspiration is accomplished by plunging a large needle or trocar and canula through the chest wall and having the instrument used attached by a tube to a vacuum pump. This method does not remove all of the pus nor the shreds of fibrinous material, and often has to be resorted to repeatedly, as proper drainage is not established.

A fter-treatment.-This consists in methods employed to bring about the full expansion of the lungs. For this purpose two bottles connected by tubing are used. One bottle is filled with water, which may be colored if convenient. The child is instructed to blow the water from 
one bottle into the other. This can be made a pastime, and it serves the purpose of expanding the lungs.

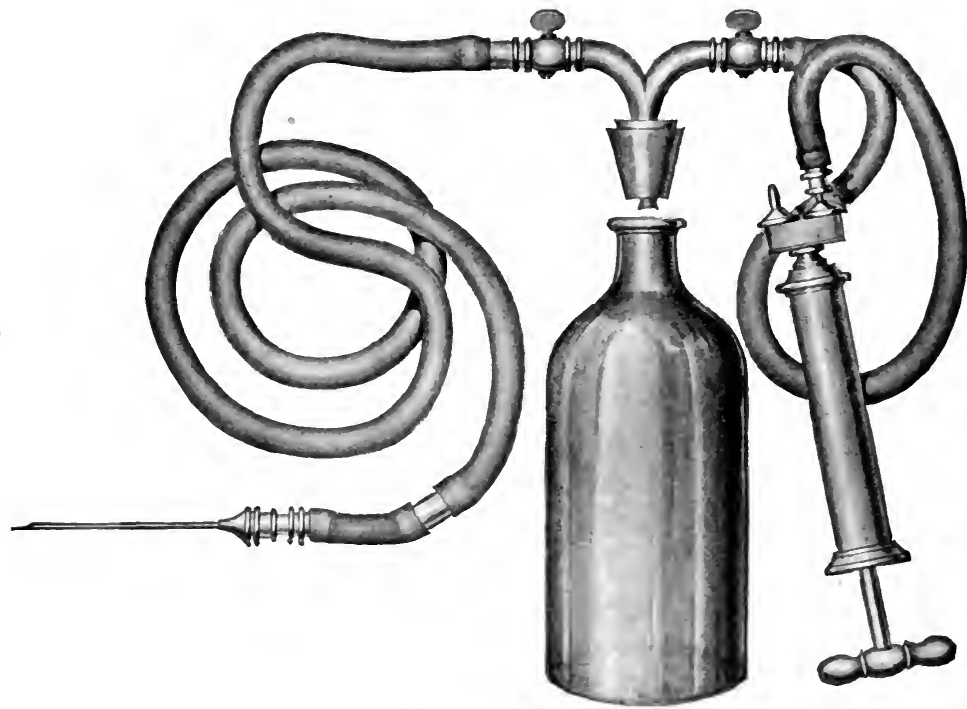

F1G. 24. $-\Lambda$ pparatus for aspiration (Kerley).

Nursing.-The temperature of the room should be maintained at $68^{\circ} \mathrm{F}$; ; ventilation is necessary; bathing should be restricted to sponging.

Sleep and feeding usually are undisturbed.

'The two precautions to be taken by the nurse in dressing a case of empyema which has been operated upon are: (I) To see that there is no danger of the tube slipping through the wound and being lost in the pleural cavity. This can be prevented by inserting a safety-pin through the end of the tube. (2) To see that the exit of the tube is covered with gauze. This covering acts as a valve 
which allows the pus to escape and prohibits air from entering. The presence of air in the pleural cavity prevents the full expansion of the lungs, which have been compressed by the fluid.

The child should be allowed to move from side to side, but should be encouraged to lie on the side where the wound is situated, as long as there is any discharge. At the end of two weeks the child can sit up.

A specimen of the pus from the wound should be obtained at the time of the operation and kept in a sterile test-tube with an aseptic cotton stopper.

The temperature, pulse, and respirations should be taken every three hours if there is fever.

The exercise of blowing water from one bottle to another should be carried out if the child is able to do it. If this fails, blowing soap bubbles is a good substitute.

Hemorrhagic Pleurisy.-This is a bloody effusion into the pleural cavity. After seven years of age hemorrhagic pleurisy is sometimes seen in connection with tuberculosis and severe anemias. It is also called hemothorax.

Diaphragmatic Pleurisy is an inflammation of the pleura covering the diaphragm. In addition to the typical symptoms of pleurisy there is apt to be extreme dyspnea and hiccough.

Pleurodynia is the name given to rheumatism of the intercostal muscles. The chief symptom is pain in the side upon deep inspiration.

Prognosis of Pleurisy.-This depends largely on the character and amount of the fluid present. The aspirating needle is a great aid to the physician in settling this question. 
In serous pleurisies the outcome is usually good. In adhesive pleurisy the outlook is favorable, but there may be some subsequent retraction and more or less impairment of the affected side. When the pleurisy is not a complication of an acute infectious disease and arises spontaneously in children over seven years of age it is usually tubercular in character. In empyema the prognosis is always grave, but recoveries often follow.

Hydrothorax is an exudation into the pleural cavity of a clear, serous fluid. It is not due to an inflammatory condition of the pleura, but occurs in the course of a general dropsic condition of the body.

Pneumothorax.-Air in the pleural cavity.

Such a condition is caused by a rupture of the lung from any cause into the pleural cavity, allowing air to gain access from that source, or by a penetrating wound of the side, which forms an avenue of entrance for the external air.

While the first condition may be one of pneumothorax, inflammation sets in very early and there is an exudation of serum; the condition then becomes pneumohydrothorax. 


\section{CHAPTER IV}

\section{DISEASES OF THE RESPIRATORY TRACT (Continued)}

\section{THE Lungs}

THE lungs consist of air-cells at the termination of the small bronchi. On cross-section, as in the illustration,

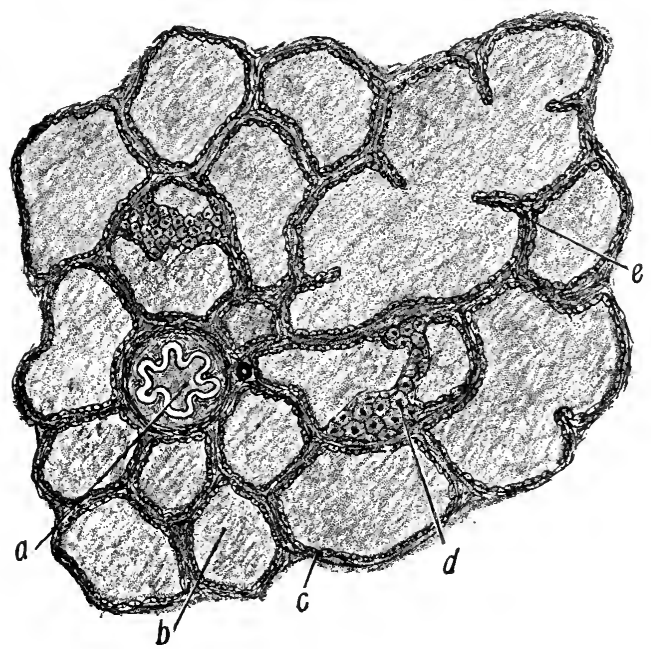

FIg. 25.-Section of lung: $a$, Cross-section of small bronchus; $b$, alveolus; $c$, alveolar wall lined with flat epithelium; $d$, top wall of alveolus entirely cut across; $e$, section into infundibulum, showing several alveoli opening into it (Leroy).

they resemble a bunch of grapes. The alveoli or air-cells have resilient walls permitting them to expand and to contract. 
In the thin walls of the air-cells are minute capillary vessels which are so arranged that the oxygen from the inspired air contained in the alveoli is readily absorbed by the blood in the capillaries; at the same time the carbon dioxid $\left(\mathrm{CO}_{2}\right)$, which is the product of the waste of the body, is thrown off from the blood into the air-cells and exhaled. This process purifies the blood, changes venous

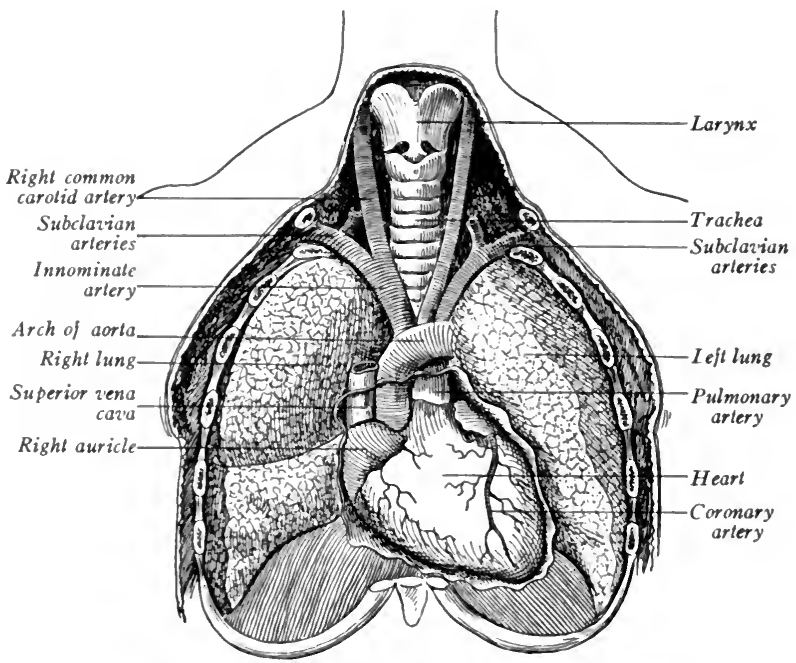

FIG 26.- Relation of lungs to other thoracic organs (Ingals).

blood into arterial, and is the sole function of the lungs.

Between the groups of air-cells there is a supporting structure of connective tissue. The air vesicles are lined with mucous membrane.

The lungs are divided into lobes: the left lung into two, the superior and inferior, and the right into three lobes, the superior, the middle, and the inferior. 
The lungs are covered by the pleura.

The respirations during childhood vary. In the newborn they are from 30 to 50 per minute, in the first year 28 to 30 , at five years 22 to 25 , at fourteen years 20 , in adult life I8 per minute.

The thin chest walls in childhood give less protection to the lungs thian those of an adult, consequently children are more affected by exposure. A distended stomach may embarrass the respiration of a child, owing to the high position of the diaphragm. Acute congestion may give rise to as severe symptoms as pneumonia, due to the small, undeveloped air-cells.

\section{CONGESTION OF THE LUNGS}

Congestion of the lungs may be active, passive, or hypostatic.

Active congestion is due to an increase in the flow of blood from the heart to the lungs. The arteries become engorged and the function of the lungs is restricted. Active congestion of the lungs is seen in conditions which produce an overactivity of the heart, such as high altitudes, excitement, and cardiac hypertrophy. In inflammatory diseases of the lungs there is an associated active congestion. The first stage of croupous pneumonia consists in this type of congestion.

Passive congestion is caused by some obstruction to the flow of blood from the lungs to the heart. It is most often caused by heart disease; under such conditions the heart is so damaged that it is unable to pump the blood with the normal force and maintain the necessary speed, the current thus becomes dammed back in the great vessels of the lungs. 
Hypostatic congestion is seen in discases which require the patients to remain for long periods upon their backs. Such protracted illnesses always weaken the heart, so that it does not have the power to force the blood column through the arteries at the normal speed and pressure. This allows the blood current to become sluggish, and in the dependent portions of the lungs there is a congestion due to an engorgement of the vessels. The reason for changing the position of a patient in bed at frequent intervals is to overcome this tendency to hypostatic congestion.

Symptoms of congestion consist of cough, dyspnea, slightly accelerated respirations, and fever.

Treatment consists in the application of some form of counterirritation to draw the blood from the congested lungs to the surface. Dry cups, mustard paste, amber oil, camphorated oil, and antiphlogistin may be used. An oiled-silk or a cotton-batting jacket at times is worn in addition.

\section{PNEUMONIA}

This term is applied to inflammation of the lungs.

The main divisions of pneumonia are bronchopneumonia, croupous pneumonia, pleuropneumonia, hypostatic pneumonia, and chronic bronchopneumonia.

Bronchopneumonia is also termed lobular pneumonia, meaning that several lobes of the lungs are involved. The pneumonic areas are small, they do not occupy a whole lobe, and are scattered through several lobes of the lungs. It is also termed catarrhal pneumonia.

Croupous pneumonia is also termed lobar pneumonia, meaning that the pneumonic consolidation usually involves the entire lobe of a lung or at least a part of one. 
It is sharply circumscribed and there are no scattered areas through the other lobes, as in bronchopneumonia. More than one lobe may be involved, the disease spreading through the additional lobe or lobes in the same manner.

The term pneumonia of the apex is used when the apices of the lungs are involved. A rare form of croupous pneumonia is double pneumonia, in which both lungs are affected, but not necessarily the whole of each. A massive pneumonia is an inflammation not only of the air vesicles, but also of the bronchi and other lung structures. A creeping or migratory pneumonia affects successively different lobes of the lungs. Epidemic pneumonia involves large numbers of children and seems to be contagious.

The contagiousness of bronchopneumonia cannot be determined without more complete data than at present existing. There seems to be no doubt, from clinical observations alone, that the secondary forms, especially those that complicate measles and diphtheria, are sometimes communicated in this way. This is probably not often true of primary cases except in hospitals for infants, where the rapid development of case after case in the same ward cannot be explained upon any other hypothesis (Holt). Croupous pneumonia is not contagious.

Under two years of age bronchopneumonia is more frequently seen than croupous pneumonia. The proportion is 75 per cent. bronchopneumonia and 25 per cent. croupous pneumonia. The reason for this is found when the structure of the lungs is studied. Before two years of age the lungs are undeveloped and their structure is mostly bronchial in character. As the child grows the air vesicles become more and more developed and the struc- 
ture of the lungs more vesicular in type. Bronchopneumonia is an inflammation of the terminal bronchi; croupous pneumonia is an inflammation of the air vesicles; pneumonia is a common disease at all ages. Therefore, before the air vesicles have developed the pneumonia attacks the predominating structures, the capillary bronchi, giving rise to bronchopneumonia; and later when the air vesicles become the principal part of the lungs the pneumonia attacks them, giving rise to croupous pneumonia.

Prognosis.-In childhood croupous pneumonia is rarely a fatal disease, while bronchopneumonia causes more deaths among infants than any other disease excepting infantile diarrhea. In the adult croupous pneumonia is one of the most treacherous and fatal diseases that exists, the mortality ranging from 20 per cent to 40 per cent. or one in every four or five dying, while bronchopneumonia is rarely met with as a primary condition.

The high mortality of croupous pneumonia in the adult is principally caused by heart failure occurring during the course of the disease. The great vascularity of the lungs (the whole amount of blood in the body passes through the minute capillaries of the lungs once in about twentytwo seconds (Vierordt)) renders it necessary that there should be no obstruction. Normally, the lungs can be likened to a sponge. It is easy, under such circumstances, for the heart to pump the blood column through the unresisting tissues. In pneumonia, however, the structure of the lungs becomes like liver. This vastly increases the obstruction and the strain upon the heart. In children the heart is strong and vigorous and it is able to cope with the extra strain, while an adult's heart has not the same inherent strength after years of work. In addition an 
adult has probably "put on flesh," every ounce of which means extra work for the heart (this is the reason that a thin person can withstand an attack of pneumonia better than a stout one). The ferocity of the disease itself seems to be worse in an adult than it is in a child, owing usually to lowered vitality from overwork and exposure. These reasons explain the difference in the mortality of croupous pneumonia in adults and in children.

Pleuropneumonia is much more frequent in childhood than in adult life.

Acute Bronchopneumonia.-Under two years of age most cases of primary pneumonia are bronchial in type. After two years of age the great majority of cases of pneumonia which follow measles, diphtheria, pertussis, and influenza are of this variety. The mortality of bronchopneumonia in children stands second only to gastro-intestinal diseases. It varies greatly, but about 40 per cent. of the cases die. This is due to the weak resisting powers of the undeveloped infant and also to the fact that when bronchopneumonia is a secondary condition it complicates such diseases as pertussis, scarlet fever, diphtheria, and influenza. These already have so weakened the child's constitution that when pneumonia sets in the child is physically unable to cope with the added infection.

Bronchopneumonia is not often seen among the better classes as a primary condition. It is a disease due to exposure and poor hygienic surroundings. Primary cases are usually due to the pneumococcus and secondary cases to what is termed a mixed infection. This mixed infection may be due to the bacilli causing the disease of which the pneumonia is a complication, such as the bacillus of 
influenza and the pneumococcus, or it may be due to the presence of the streptococcus and staphylococcus (pus organisms). The latter is the usual cause.

Bronchopneumonia generally begins in the larger bronchi, gradually working into the smaller tubes and finally involving the air vesicles. It is easier to understand bronchopneumonia if it is considered as a bronchitis of the terminal bronchial tubes and adjacent air vesicles. As in bronchitis, there is an inflammation of the small bronchial tubes with congestion and swelling of the mucous membrane, an over-secretion of mucus, and a shedding of the lining epithelial cells. This exudate fills the small air-cells adjacent to the inflamed bronchial tube, producing a pneumonic consolidation. The presence of the mixed infection causes this consolidation, composed of debris, to break down and suppurate, and thus pin-point abscesses are formed in the pneumonic area. These areas are scattered; as the disease extends from the bronchioles, only the air vesicles adjacent to the inflamed tubes are involved. The most frequent location of the disease is in the lower lobes, posteriorly, of both lungs. The healthy portions of the lungs are distended to accomplish their added task and hence a condition of hypertrophic compensatory emphysema co-exists. There are no definite stages, as in croupous pneumonia.

Symptoms. - The clinical picture of bronchopneumonia is an exceedingly varied one; there is no typical course. The symptoms most frequently seen are as follows:

The symptoms of bronchitis, if present, become slowly or rapidly worse and merge into those of pneumonia. More often in primary bronchopneumonia the onset is sudden, the child is seized with vomiting and high fever: 
cough, accelerated respiration, prostration, and cyanosis. The temperature is remittent in type. It is high, but has wide daily fluctuations of from four to five degrees. This high temperature continues for a week or two and then falls by lysis. Lysis is a gradual fall of the temperature to normal, taking from two days to a week to reach this point. In contrast to this, crisis is a sudden drop from an exceedingly high temperature to normal within twentyfour hours.

\begin{tabular}{|c|c|c|c|c|c|c|c|c|c|c|c|c|c|c|c|c|c|}
\hline$P$ & $R$ & $T$. & $\begin{array}{c}\text { ' } \\
\text { m. } e\end{array}$ & $\begin{array}{c}2 \\
m \cdot e\end{array}$ & 3 & $\mathrm{ln}$ & & $\begin{array}{c}5 \\
m \cdot e\end{array}$ & $\begin{array}{c}6 \\
m . e\end{array}$ & $\begin{array}{r}7 \\
m\end{array}$ & & & $\begin{array}{c}9 \\
\text { m.e. }\end{array}$ & $\begin{array}{c}10 \\
m \cdot e\end{array}$ & " & $\mathrm{m}$ & \begin{tabular}{|l}
13 \\
m.e
\end{tabular} \\
\hline 170 & 70 & 108 & & & is & & & & & & & A & & & & & \\
\hline 160 & 65 & 107 & & & 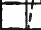 & & & & & & & i & & & & & \\
\hline 150 & 60 & 106 & & T & & & & & $i$ & & $5 i$ & & & & & & \\
\hline 140 & 55 & 105 & $e_{1}^{n}$ & & & & & $A$ & $i$ & & & & is & & & & \\
\hline 130 & 50 & 104 & & & & 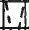 & & ii & f & if & & & & & & & \\
\hline 120 & 45 & 103 & 1 & & V & 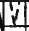 & & $i$ & & $i$ & & & 1 & & & & \\
\hline 110 & 40 & 102 & & & & & & & & 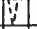 & & & 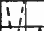 & . & & & \\
\hline 100 & 35 & 101 & & $\pi$ & $\nabla$ & $\nabla$ & & & & & & & ? & & & & \\
\hline 90 & 30 & 100 & & $\nabla$ & & & & & & & & & & & $\forall$ & $v$ & $\therefore$ \\
\hline 80 & 25 & 99 & & & & & & & $i$ & & & & 1 & & & & \\
\hline 70 & 20 & 98 & & & & & & & & & & & & & & & \\
\hline 60 & 15 & 97 & & & & & & & & & & & & & & & \\
\hline
\end{tabular}

Fig. 27.-Chart of the temperature (-) and respirations (-..) in bronchopneumonia. Child one year old (Kerr).

Just before death the temperature often reaches $107^{\circ} \mathrm{F}$. and $109^{\circ} \mathrm{F}$.

The respirations average from sixty to eighty per minute, often they are one hundred per minute, and occasionally a hundred and twenty. There is great shortness of breath (dyspnea), the child struggles for each breath, the chest is retracted at the base, and the other symptoms of dyspnea are present. The respiratory action is more affected than the heart action, and if the child succumbs it is usually by respiratory failure, the symptoms of which are very rapid, superficial breathing, sometimes 
a hundred to a minute, blueness of the lips and finger-nails, and often a bluish hue to the body.

The pulse averages from one hundred and fifty to two hundred per minute. When very rapid it is often irregular. The character of the pulse is more important than the rapidity. At first it is full and strong, but later it becomes weak, thready, compressible, and intermittent.

Cough is always present and very persistent, more so than in croupous pneumonia. A good, strong cough is not an unfavorable symptom, as it shows that the reflex irritability of the bronchial tubes is still present. When this is lost the mucus is not removed, the lungs fill up, and respiratory failure threatens. Suppression of cough is, therefore, a bad sign.

There is no expectoration before four years of age, the mucus is either swallowed or re-inspired. During severe paroxysms of coughing, if the child be turned on its face or inverted, much of the mucus will run out of the mouth.

A blueness (cyanosis) of the skin and mucous membrane is found in severe cases. It is duc to a sudden congestion of a portion of the lungs not previously affected. Even when present only at lips and finger-tips, the patient should be very carefully watched, and if further symptoms of respiratory failure develop, they should receive prompt treatment (sce pages 100 and 394).

Prostration is progressive; at first it may be moderate, but in the final stages there may be symptoms which are known as the typhoid state. These are delirium, picking at the bed-clothes (carphalogia), twitching of the tendons (subsultus tendinum) rare in childhood, and dry, brown, fissured tongue.

Gastro-intestinal Symptoms.-Often there are from four 
to six green stools a day, containing mucus and undigested food, due to the weakened digestion from the fever and induced by feeding improper food. This same condition may cause vomiting. Vomiting and diarrhea add much to the danger of the attack, and when the result is in doubt, may turn the scales against the patient. In summer this complication is more frequent and more severe. Distention of stomach and intestines from gas may cause attacks of cyanosis, which condition should be relieved as soon as possible. The rectal tube may be employed with care.

The urine is scanty.

Complications.-Pleurisy is nearly always present. Purulent meningitis sometimes complicates acute bronchopneumonia, but the most frequent complications are referable to the gastro-intestinal tract.

Croupous pneumonia is an acute, infectious, inflammatory disease of the lungs characterized by a high fever and ending by crisis in from five to nine days. Seventyfive per cent of the cases of croupous pneumonia are caused by the diplococcus pneumoniæ.

The term lobar pneumonia is generally used for this form of pneumonia, so-called on account of its tendency to involve a whole lobe of the lung in contradistinction to bronchopneumonia, which is sometimes called lobular pneumonia.

Croupous pneumonia is one of the oldest recognized diseases; it was described fairly accurately by Hippocrates in $460 \mathrm{~B}$. C.

In childhood pneumonia follows, in a general way, the character of an attack in the adult. In speaking of bronchopneumonia it was said that it was the pneumonia of early infancy. This is true until children are about 
two years of age, after which they are usually attacked by croupous pneumonia. This disease has a tendency to attack children that were previously healthy; it is especially prevalent in the spring of the year.

Epidemics are not frequent among children, and the disease is rarely fatal. In the order of frequency the disease attacks the following portions of the lungs: left base, right apex, right base, left apex.

The complications of pneumonia are pleurisy, endocarditis, meningitis, and neuritis.

Children rarely have complications, the one most often seen being empyema, which is probably on account of the proneness of children to have severe pleurisy associated with croupous pneumonia. The temperature is generally higher, the pulse more rapid, the duration shorter, and the cerebral symptoms more frequent in children than in adults, otherwise, as has been mentioned before, the disease is the same.

The cause of croupous pneumonia is usually exposure. The disease occurs more frequently in males than in females. It is usually primary, occasionally it will complicate some form of infectious disease.

There are four distinct stages in croupous pneumonia: The stage of congestion or engorgement, seen in the first twenty-four hours. The stage of red hepatization, of from four to five days' duration. The stage of gray hepatization, of from six to ten days' duration. The stage of resolution, of from six to ten days' duration.

Congestion is the stage in which the lung is engorged with blood, yet permeable to air. It is an active congestion of the lungs.

Stage of red hepatization. The term hepatization is 
given on account of the liver-like appearance of a lung on section. In this stage the lung is dark red in color, and of very firm consistency. This is caused by the air-cells being filled with what is known as a croupous exudate. This exudate is composed of red blood-corpuscles from the capillaries surrounding the alveoli and exfoliated epithelial cells which line the walls of the air vesicles, all massed together by fibrin. The croupous exudate excludes the air from the alveoli affected. This gives rise to a consolidation of the lung, which normally is permeable to air. This condition is called a pneumonic consolidation. The size of the area consolidated depends upon the number of air-cells filled with the croupous exudate. In croupous pneumonia a whole lobe or more is usually affected. It can be understood to what extent the function of the lungs would be impaired under such conditions, and also the tremendous extra strain thrown upon the heart, which has to pump the blood through the consolidation just as through the normal lung.

The stage of gray hepatization is so called from the appearance of a lung on section. It is grayish and still firm and liver-like. The grayness is due to the air-cells being filled with white blood-corpuscles, the red bloodcorpuscles and fibrin having been withdrawn. The pneumonic consolidation still remains, as the affected area is still impermeable to air. The whole pneumonic consolidation has become softened in this stage by degeneration, and is in preparation for the stage of resolution.

The stage of resolution is characterized by the liquefaction of the croupous exudate, part of which is expectorated and part absorbed. Resolution generally begins when the temperature falls to normal and lasts about a 
week. Delayed resolution is the term applied to a slowly resolving pneumonia, which may be prolonged from a week to a month.

The pleura adjacent to the pneumonic area is nearly always involved.

Symptoms.-The disease is ushered in suddenly with high fever, prostration, acceleration of the respiratory rate, and increase in the pulse-beats. In children vomiting often attends the onset. Pain in the side is also quite

\begin{tabular}{|c|c|c|c|c|c|c|c|c|c|c|c|c|c|c|c|}
\hline & & & 1 & 2 & 3 & 4 & 5 & 6 & 7 & 8 & 9 & 10 & 11 & 12 & 13 \\
\hline$P$. & $R$. & T. & m.e. & me.e. & mr.e. & mor. $e$ & m $e$ & m. e & mer.e & m.e & mm. & $m \cdot e$ & m. e & $m . e$ & m. e \\
\hline 170 & 70 & 108 & & & & & & & & & & & & & \\
\hline 160 & 65 & 107 & & & & & & & & & & & & & \\
\hline 150 & 60 & 106 & & & & & & & $i$ & & & & & & \\
\hline 140 & 55 & 105 & & & & & & & $!:$ & & & & & & \\
\hline 130 & 50 & 104 & & & & & & & & & & & & & \\
\hline 120 & 45 & 103 & & & & & & & & & & & & & \\
\hline 110 & 40 & 102 & $7:$ & & i & & & 1 & & & & & & & \\
\hline 100 & $35^{\circ}$ & 101 & 1 & $y$ & $\because$ & 2 & $\bar{y}^{\prime}$ & & $y$ & & & $\therefore$ & & & \\
\hline 90 & 30 & 100 & 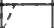 & & & & & & & & & 6 & $y^{\prime}$ & $\because$ & \\
\hline 80 & 25 & 99 & & & & & & & & & & & & & \\
\hline 70 & 20 & 98 & & & & & & & & & & V & 7 & 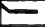 & \\
\hline 60 & 15 & 97 & & & & & & & & & & & & & \\
\hline
\end{tabular}

FiG. 28.-Chart of the temperature (-) and respirations (-.-) in lobar pneumonia. Child one year old (Kerr).

common; a decided chill is not as characteristic as it is in adults. At times the pain is referred to the region of the appendix and appendicitis is simulated. The child appears profoundly sick from the beginning. The skin is hot, the face flushed, often more so on the side corresponding to the pneumonic consolidation. The temperature reaches $104^{\circ}$ to $105^{\circ} \mathrm{F}$. within twenty-four hours. The pulse is full and strong, averaging $\mathrm{I} 20$ to $\mathrm{s} 30$ per minute, the respirations are labored and from 40 to 50 per minute, which in severe cases may be as high as 80 to 1oo. The signs of dyspnea are present, the breathing is not always 
regular, and there is a characteristic catch of the breath or moan at the end of each expiration. Cough develops early and is hard, catchy, and partially suppressed. There is no expectoration. The urine is scanty.

The temperature remains continuously high with slight daily fluctuations. Herpes develops on the lips, the child may be delirious, more often at night than at any other time. Cyanosis may be present, but it is not nearly so frequent as in bronchopneumonia. The danger of respiratory failure is practically absent; on the other hand, the chances of heart failure are vastly increased.

In a day or two the cough becomes loose. In children under four years of age there is no expectoration, as the mucus is swallowed. Older children may have the rusty sputum seen in adults.

The position in bed (decubitus) is also characteristic; the patient will lie on the side affected to give the sound side a chance for increased expansion. Leukocytosis is usually present, the white blood-corpuscles being increased from 6000 to 10,000 or 20,000 or more, per $\mathrm{cmm}$.

All these symptoms continue unchanged for from five to nine days, when, if recovery takes place, a sudden drop in the temperature occurs, often accompanied by free perspiration, while a state of comparative comfort succeeds to that of great distress, and it may be followed by a long and refreshing sleep. This is known as the crisis. It may be preceded by a fall of temperature a day or two earlier, which is again followed by a rise. If there is a fall of this description it is called pseudocrisis. The fall in a crisis is sometimes as much as seven degrees in a single twenty-four hours, and the minimum is often slightly subnormal, from which it rises rapidly to the normal. 
Sometimes the temperature falls by lysis. From this point onward convalescence is rapid; in a week the child is out of bed and in a month is out of doors.

Pneumonia either ends in resolution, abscess, gangrene, interstitial or fibroid pneumonia, phthisis, or, if fatally, usually by heart failure. Abscess from introduction of pus organisms; gangrene from engorgement of pulmonary vessels; interstitial pneumonia from overgrowth of connective tissue from exudate becoming organized; phthisis from introduction of tubercle bacilli.

The symptoms of heart failure are coldness of hands and feet, then of the legs and arms, a rapid, compressible, and sometimes irregular pulse, muscular weakness and pallor, but usually no cyanosis.

Death usually occurs at the time of the crisis, so if the child can be kept alive until this time has passed, it is practically saved.

Prognosis.-Mortality is about 4 per cent. The difference from pneumonia in adults is at once apparent when it is known that the adult mortality is from 20 per cent. to 40 per cent. When complicated by meningitis and endocarditis it is usually fatal.

Cerebral pneumonia is a form of the disease characterized by severe nervous symptoms. Convulsions occur in about 5 per cent. of the cases, and in the more severe forms arching of the back (opisthotonus) may be found.

Pleuropneumonia.-Children are especially prone to have pleurisy, and nearly every case of pneumonia could be called pleuropneumonia. Usually under this term are included cases with excessive amount of pleurisy, the two processes uniting to form a single clinical type of disease. There is little to distinguish a case of pleuropneumonia except the severity of all the constitutional 
symptoms. The temperature is often higher, the prostration greater, and the patient in every way impresses one as being more seriously ill than with ordinary pneumonia.

Hypostatic pneumonia, like hypostatic congestion, is caused by the venous stasis, owing to the child's recumbent position. For this reason the position of a patient in bed should be frequently changed.

Aspiration pneumonia is due to the inhalation of some foreign substance into the lungs, which gives rise to an inflammation. Such foreign material may be diphtheric membrane, food, etc. The symptoms are those of croupous pneumonia.

Chronic interstitial pneumonia, as said before, is sometimes a sequel to croupous pneumonia, but in children it is usually associated with phthisis. It is due to an overgrowth of fibrous tissue, with subsequent retraction of the lung tissues. It is generally characterized by chronic cough, slight dyspnea, and scanty expectoration. Bronchiectasis sometimes results when there is the characteristic fetid sputum, which occurs in gushes.

Gangrene of the lung is sometimes seen following pneumonia. The bacteria of putrefaction gains access to the diseased area and cause necrosis. It is fatal.

Symptoms.-Children have the characteristic symptoms of inflammatory disease of the lungs, such as cough and dyspnea, together with profound prostration and the expectoration of very offensive sputum.

Abscess of the lungs is more common than gangrene. Small abscess may be seen in bronchopneumonia. Sometimes an empyema (purulent pleurisy) will rupture into the lungs, causing a secondary abscess. 
The symptoms here will be those of any lung affection (fever, cough, dyspnea, and expectoration) plus the characteristic symptoms of pus, namely high and irregular fever, rigors, sweats, and pallor.

Embolic septic pneumonia or a metastatic abscess of the lung is caused by a septic embolus. Such a septic embolus may arise at the seat of some putrid inflammation or suppuration, such as a wound of an operation or a compound fracture. This embolus lodges in the small capillaries of the lung and starts a point of suppuration, from which there will arise all the symptoms of pus. It is generally only one of the lesions of pyemia.

Treatment.-Pneumonia, both bronchopneumonia and the croupous form, are diseases which for a favorable outcome depend not so much on the remedies given as upon the general hygienic measures employed. These measures are grouped under the term general nursing. Since in the treatment of bronchopneumonia very little can be done for the disease and very much can be done for the patient, and since croupous pneumonia is a self-limited disease having a strong tendency in childhood to recovery regardless of the treatment adopted, the plan of treatment of both diseases is practically the same.

Nursing. - The indications are, so far as possible, to make the child comfortable during his illness, to prevent complications, and to treat the individual symptoms as they arise. Bronchopneumonia is frequently a complication of one of the infectious fevers, such as measles, whooping-cough, and influenza; so in the nursing of these conditions prophylactic measures must be employed.

Perhaps in the majority of cases of pneumonia in childhood hygienic treatment is all that is required. The patient should be kept in a large well-ventilated room, 
and, if possible, changed from one room to another two or three times a day, to allow thorough airing. Some physicians adopt the open-air treatment for pneumonia. The child's bed is then placed on the porch or veranda. When these do not exist, the bed is drawn in front of an open window. The child is protected from the wind, a flannel cap and mittens are worn, it is covered with blankets, and surrounded with hot-water bottles.

Whenever it is necessary to change any of the clothing or to give baths, the child is brought into the warm room. The bed is thoroughly warmed with hot-water bottles before the patient is again placed in it. Older children should be kept in bed; infants can be held in the nurse's arms for a considerable part of the time. A frequent change of position is essential. The bed-covers should never be tucked in tightly. Food should be given at regular intervals, and when the child is restless, fretful, sleepless, or nervous, sponging with tepid water usually makes him comfortable. Severe nervous symptoms require the application of ice, either in the form of a cold bath or an ice-bag. Pain is usually relieved by the application of mustard paste or turpentine stupes. In bronchopneumonia an oiled-silk jacket is sometimes worn throughout the attack, and, if necessary, counterirritation maintained by mustard paste. Hot poultices of flaxseed may be employed. When new poultices are applied the old poultice is first rolled back from one side, and the new one is placed in position; then the other side is covered in the same way. This prevents exposure. Amber oil is also useful.

Fever in itself means nothing, as it only indicates the severity of the lesions. Since a temperature of $105^{\circ} \mathrm{F}$. is characteristic of pneumonia, it is not necessary to do 
much for it unless it become higher. The nervous symptoms call more often for treatment than the fever, and as the two go hand in hand, it is customary to keep the fever under control. The best means for this end is cold. It may be used by a graduated bath for small children $_{b}$ a cold pack for older ones, or a simple sponging and an ice-bag. Some physicians use only warm baths $\left(100^{\circ} \mathrm{F}.\right)$ in their treatment of pneumonia.

Iodin is often applied to the chest in unresolved pneumonia. If the ordinary tincture of iodin causes blistering of the skin, it can be diluted one-half with alcohol.

It is the emergencies which arise in pneumonia with which the nurse has to contend. In respiratory failure there is great dyspnea, cyanosis, and signs of collapse. The physician should be immediately summoned; in the meantime, if the child shows great distress in breathing while in the recumbent posture, it should be propped up in bed, oxygen should be administered; gentle friction of the sides of the chest at times stimulates the respiratory muscles. He may order a mustard tub and hypodermic injections of one or more of the following drugs: atropin, caffein, strychnin, and nitroglycerin. In sudden attacks of great cyanosis a mustard tub is advantageous.

As a moist atmosphere is the best for pneumonia, a croup tent with steam atmosphere medicated with compound tincture of benzoin generally relieves the dyspnea, especially if there is much bronchitis associated with the pneumonia. In an ordinary case a child should remain in bed for about a week after the normal temperature has been reached.

The temperature, pulse, and respirations should be taken every three hours. 


\section{CHAPTER V}

\section{DISEASES OF THE DIGESTIVE TRACT}

ThE digestive tract is composed of the mouth, tonsils and pharynx, the esophagus, stomach and intestines, the pancreas, and the liver.

The symptoms of the diseases of the digestive tract are exhibited in the condition of the tongue, breath, appetite, and the stools. Vomiting, pain, tenderness, and distention of the abdomen are associated symptoms.

The tongue often has a light, uniform coat in health, especially in bottle-fed babies. It becomes heavily coated upon the slightest indication of indigestion. The mucous membrane covering the tongue is the only part of the lining of the alimentary canal visible, and indicates the condition of the mucous membrane in the lower digestive tract. The tongue also becomes coated in fever and catarrhal conditions of the nose and throat. In several diseases there is a characteristic appearance of the tongue.

In typhoid fever, in the early stages, the tongue is red and it often trembles upon extrusion, and later becomes dry, brown, and fissured.

In scarlet fever, in the first stage, the tongue is heavily coated with a whitish fur, with the exception of the tip, which is red. This whitish fur gradually peels off, so that in a day or two the tongue is a deep red, with the papillæ deeply injected. This has given rise to the name strawberry tongue, characteristic of this disease. 
$\mathrm{IO} 2$

In gastro-enteritis sometimes the tongue is gray-coated and flabby, with an oval bare spot in the center, which is red and glossy. In older children scars on the tongue are due to tooth-wounds inflicted during epileptic convulsions.

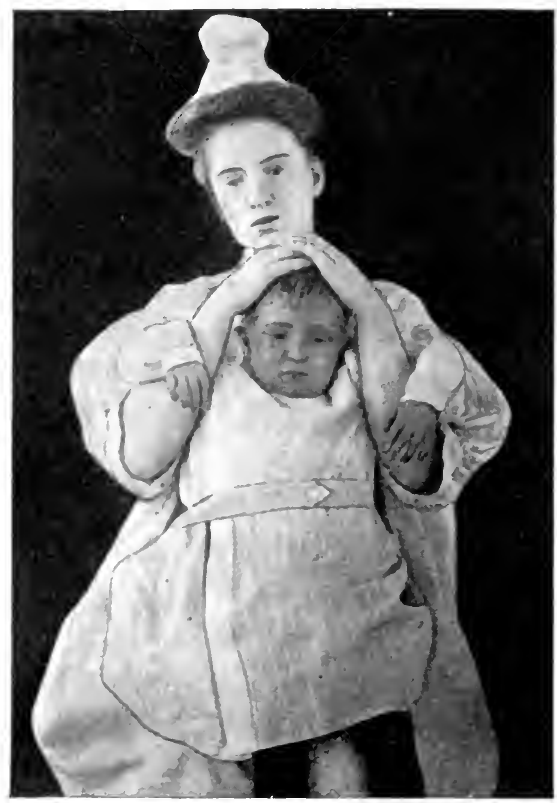

FIG. 20.-Illustrating a very good and common position for mouth and throat examination (Kerr).

Fetor of the breath is frequently due to some local condition, such as chronic rhinitis, tonsillitis; to retained particles of food; to caries of the teeth; to certain lung diseases; to dyspepsia; and to the ingestion of certain drugs.

'The condition of the appetite may be inordinate, lost, or perverted. The following names have been applied to the different varieties of appetite: 
Bulimia is the term applied to an inordinate appetite.

Anorexia is the term applied to loss of appetite.

Pica is a craving for unnatural foods.

Dysphagia or difficulty in swallowing may result from local inflammations, stricture of the esophagus, or paralysis.

Malformations.-In infants these are not uncommon. The conditions most frequently seen are hare-lip, cleft

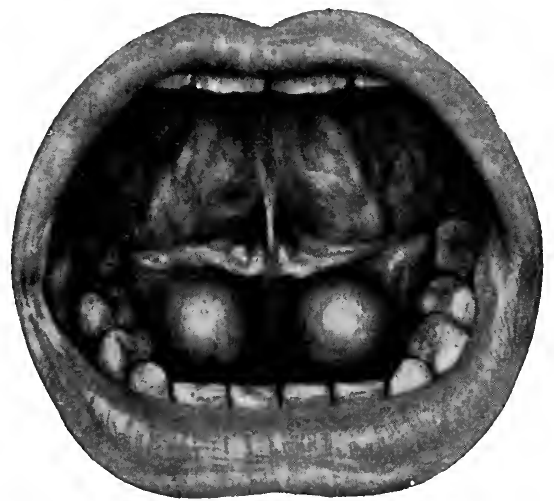

Fig. 30.-Ranula. The growth lies in the middle of the mouth and seems to be divided into two parts by the constriction of the frenum (from Grünwald, Diseases of the Oral Cavity).

palate, and tongue-tie. A large protuberant tongue is seen in cretinism. The principal difficulty experienced in such malformations is in feeding. The child is unable to grasp the nipple and feed properly. Not enough food is obtained, and they die from inanition unless the deformity is corrected.

Ranula is a cystic tumor of the floor of the mouth due to degeneration of the sublingual gland or its excretory duct. At times it interferes with swallowing. 
Feeding in Malformations of the Mouth.-Harelip.-Here it is impossible for the child to suck. Often, if the cleft is held together by the fingers, the child can nurse. If this fails, milk is given by means of a medicine dropper.

Cleft Palate:-Here the roof of the mouth is the part lacking; this also makes sucking impossible. At times will be found useful a special nipple consisting of a broad flap of rubber upon the upper surface, which fills the gap in the roof of the mouth and thus makes sucking possible. Sometimes an inverted bowl of a spoon placed in the cleft will answer the same purpose. If these methods fail it is necessary to resort to the medicine dropper.

Tongue-tie.-This condition sometimes prevents proper sucking. In the same way the large protuberant tongue of cretinism acts as a barrier. The medicine dropper may have to be resorted to in these cases.

If for any reason the medicine dropper is unsuccessful in supplying the child with sufficient nourishment, gavage may be practised (see page 4I6).

\section{Diseases of THE Mouth}

Ulcer of frenum is seen in cases of pertussis. It is a small ulcer on the frenum caused by the propulsion of the tongue against the teeth during coughing. It is easily cured by touching with alum.

Glossitis is the name given to inflammation of the tongue.

Gingivitis is inflammation of the gums. 


\section{STOMATITIS}

This disease is very common among the poorer class of patients. It is due to uncleanliness and to a spongy condition of the mouth seen in ill-nourished children. There are several varieties named according to the appearance of the lesions in the mouth.

Catarrhal Stomatitis.-This is a swelling of the mucous membrane of the mouth. The membrane is red and injected, the saliva is increased, and either dribbles

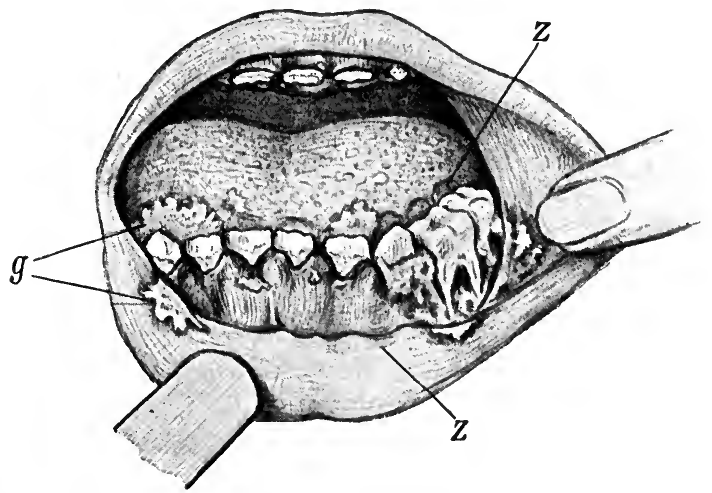

Frg. 31.-Ulcerative stomatitis in a child four years of age: $Z$, Tooth-marks on the tongue and mucous membrane of the lips; $g$, ulcers (Frühwald and Westcott).

from the mouth or is swallowed. It hurts the child to nurse, and as a consequence food is rejected. There is, in addition, restlessness, languor, and some fever.

Aphthous Stomatitis.-In this condition the mucous membrane is swollen and red. There are small, round vesicles on the tip of the tongue, on the inner side of the lips, and on the cheeks. There may be only a dozen vesicles present, or the whole mucous membrane may be covered. These little vesicles break and leave small, 
shallow ulcers having a red rim. 'The other symptoms of stomatitis common to all varieties are present. They are dribbling of saliva, heat and pain in the mouth, refusal of the child to nurse, restlessness, languor, and fever.

Ulcerative Stomatitis.-In addition to the general symptoms of stomatitis there is an ulceration of the mucous membrane beginning at the angle formed at the junction of the cheek and the alveolar processes, which is the portion of the jaw that holds the roots of the teeth. The glands under the jaw are enlarged, but do not suppurate.

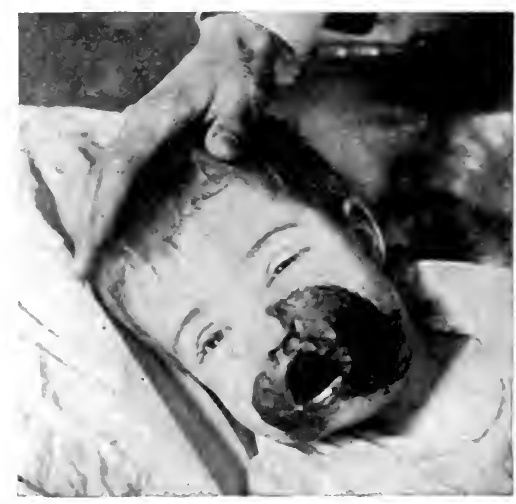

Fig. 32.-Noma of the face (case of A. T. Bazin).

In severe cases necrosis of the jaw may follow, with a subsequent loosening and falling out of the teeth.

Parasitic Stomatitis (Thrush).-The general symptoms of stomatitis are present. On inspection numerous milk-white elevations are found, which on removal leave a raw surface. This is due to a fungous growth introduced through dirty nipples.

Gangrenous Stomatitis or Noma.-It is usually seen 
in debilitated children between the ages of two and six years, and generally follows one of the specific fevers, such as measles or whooping-cough.

The symptoms of stomatitis are marked. The cheek, the part affected, is swollen, hard, red, and glazed externally; and internally there is noted an irregular, sloughing ulcer. There is destruction of the tissues and in some cases the skull is exposed. In the large majority of cases the end is death. If there is recovery, deformity is present except in a few rare cases in which the ulcer does not perforate the skin.

Prophylaxis.-Always keep the mouth clean in any illness, especially in infectious fevers.

Mercurial stomatitis is caused by the unnatural susceptibility which certain children have toward mercury. It causes soreness of the teeth and redness of the gums. If the mercury is continued, necrosis of the jaw sometimes results.

Treatment.-In the treatment of stomatitis the chief thing to do is to keep the mouth clean by the frequent applications of antiseptic washes. In mercurial stomatitis, of course, stop the mercury. Calomel may produce this condition in the very susceptible.

The best mouth wash is boric acid, about Io gr. to the ounce. Care should be taken to sterilize thoroughly the nipples of the bottles before feeding, and a bottle should never be handed from one baby to another. Powdered burnt alum applied to ulcers usually causes prompt recovery.

In feeding, the milk should be given cold, by the dropper, if necessary. Cool substances relieve the pain and heat in the mouth; therefore, cracked ice is acceptable. Fruit 
and vegetables should be given to older children to counteract the malnutrition, and in children suffering from scurvy orange juice should be given frequently.

In severe cases of stomatitis gavage may be necessary (see page 4 I6).

\section{TONSILLITIS}

Tonsillitis is not common in infancy, but throughout childhood it is often seen. It is more common in those of a rheumatic tendency and in children who have enlarged tonsils. Exposure to cold and wet while the child is in a run-down condition is the usual cause.

There are three varieties: simple or catarrhal, follicular, phlegmonous, or quinsy.

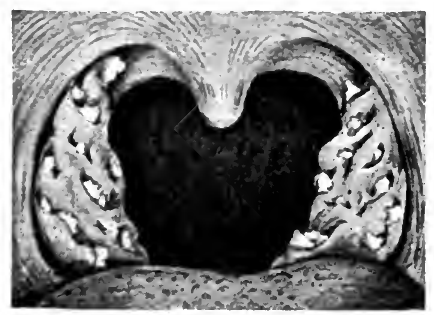

FIG. 33.-Chronic follicular tonsillitis (Frühwald and Westcott).

Symptoms.-In children the constitutional symptoms are more prominent than the local. The attack comes on suddenly, often with a chill and romiting. The temperature rapidly reaches $102^{\circ} \mathrm{F}$. to $103^{\circ} \mathrm{F}$, and in severe forms $104^{\circ} \mathrm{F}$. to $105^{\circ} \mathrm{F}$., and there is marked prostration. The tonsils are swollen and red, and there is some pain and difficulty in swallowing. The glands at the angle of the jaw are enlarged, but the swelling is not pronounced. 
In the catarrhal form the tonsils are swollen, red, and covered with tenacious mucus.

In the follicular form, sometimes called lacunar angina, in addition to the above symptoms there are numerous yellowish-white spots on the tonsils. These are due to the follicles or crypts being filled with mucus and exfoliated epithelial cells. These can often be pressed out as little plugs (see Fig. 33).

In quinsy, which is comparatively rare in childhood, there is an abscess present in the tissues immediately surrounding the tonsils. This causes extreme swelling of the tonsils, often so much so that the space between the two is almost closed. The local symptoms are more prominent than the constitutional in this condition. The fever is not apt to be over $102^{\circ} \mathrm{F}$. and there is not as much prostration as in follicular tonsillitis. At the same time there is much more pain and difficulty in swallowing. The gland affected soon softens, fluctuates, and ruptures. It is almost always unilateral in childhood. At its height swallowing is almost impossible, the voice is lost, and breathing is difficult.

Treatment.-Pellets of ice and a gargle of chlorate of potash, I dr. to a pint of water, give relicf in tonsillitis, and frequently the patient can be rendered more comfortable by the external application of an ice-bag, a poultice, or iodin over the angle of the jaw.

\section{CHRONIC HYPERTROPHY OF THE TONSILS}

Repeated attacks of acute tonsillitis lead to a permanent enlargement of the tonsillar tissues called hypertrophy. The tonsils intrude into the passage leading to the pharynx. In severe cases they may almost meet. This causes ob- 
struction to the passage of food and leads to a chronic catarrh of the throat.

Nearly all cases of enlarged or hypertrophied faucial tonsils have associated with them an overgrowth of the lymphatic tissue in the pharynx behind the posterior openings (nares) of the nose. This is called an adencid. The two conditions together give rise to characteristic symptoms.

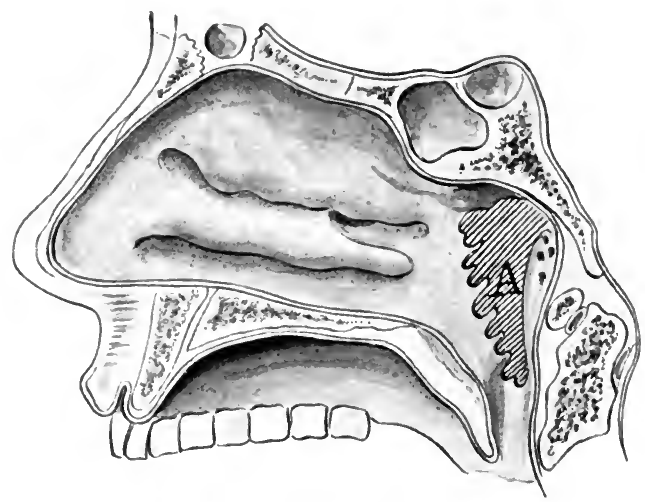

FIG. 34.-Diagram (anteroposterior) illustrating by the shaded portion $(A)$ the situation of adenoid vegetations in the nasopharynx (Kerr).

The child sleeps poorly, is restless, and snores. It breathes through the mouth and there is constant catarrh of the nose and pharynx. From the fact that the catarrh can easily extend into the Eustachian tube, which runs from the internal ear to the upper part of the pharynx, attacks of middle-ear disease (otitis media) are quite common, and deafness may result. The child is poorly nourished and is subject to acute attacks of tonsillitis. It also predisposes to diphtheria and scarlet fever. 
Treatment.-The best plan is to have the tonsils and adenoids removed if the symptoms are sufficiently annoying.

Operation.-According to the size of the tonsils and the

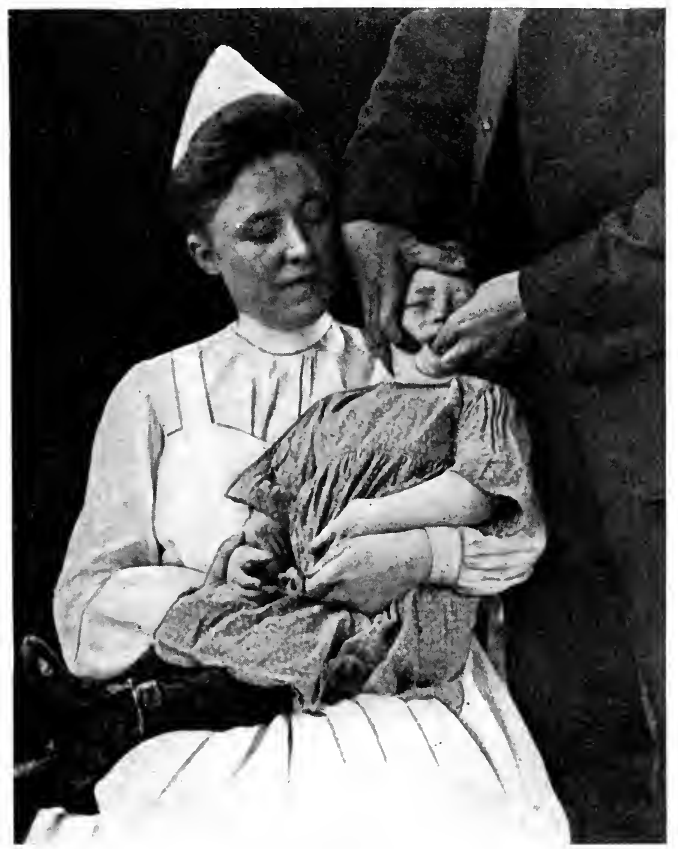

Fig. 35.-Examination of the nasopharynx for adenoi 1 ve zet 1 tions. (The examiner in this instance is left-handed.) (Kerr.)

preference of the operator, several methods may be employed. The tonsils may be dissected out, removed with a snare, cut off by means of an instrument called a guillotine, or by means of "biting" forceps. The tonsils are cut off wherever desired, and the pharyngeal tonsils or 
adenoids removed at the same time. The operation is without danger. The secondary hemorrhage can be controlled by pellets of ice, pressure, or cotton containing some styptic, like Monsel's solution. It is usually a permanent cure. In a few instances the tonsils again hypertrophy after removal.

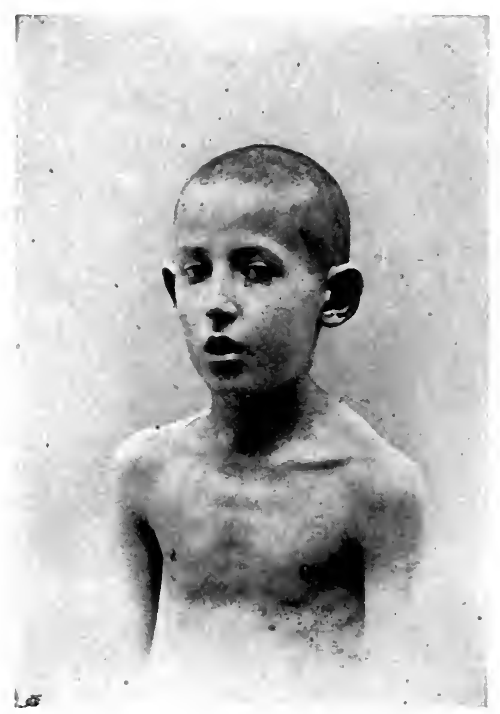

Fig. 36.-Typical appearance in adenoid vegetations: Boy ten years old (Frühwald and Westcott).

The nasopharynx is usually irrigated with normal salt solution after the operation, and cold things to eat, like ice-cream, are most acceptable.

\section{PSEUDODIPHTHERIA OR VINCENT'S ANGINA}

This is the name given to an ulceromembranous form of inflammation which attacks the mucous membrane of the 
mouth and tonsils. It is characterized by the formation of a membrane, yellowish-gray in appearance, and very offensive. When this membrane is stripped off it leaves a raw, bleeding surface behind. In twenty-four or fortyeight hours a deep, punched-out ulcer forms, with injected edges. The constitutional symptoms are mild. The temperature ranges between $100^{\circ} \mathrm{F}$. and $102^{\circ} \mathrm{F}$. In three or four days the ulcer gradually heals.

Differential Diagnosis. - The appearance of this condition is very similar to diphtheria, especially when the fauces and tonsils are the seats of the inflammation. The bleeding which accompanies the stripping of the membrane is very misleading. The differential diagnosis can be determined only by an examination, under the microscope, of a smear, properly stained, taken from the lesion. The Klebs-Löfler bacillus is not present, but two characteristic bacilli are found. They are called spirillum and fusiform, from their shape. The disease is only mildly contagious, if at all.

The necessity of making an absolute diagnosis, by means of microscopic examination, between Vincent's angina and true diphtheria is of the greatest importance. True diphtheria is subject to quarantine; Vincent's angina is not; diphtheria is a very grave disease; Vincent's angina usually is mild, although in severe cases noma and septicemia may develop. Finally, in diphtheria others should immediately receive antitoxin, as well as the patient. This is not necessary in Vincent's angina.

\section{PHARYNGITIS}

-Pharyngitis is an acute sore throat, or, more particularly, an acute inflammation of the mucous membrane 
lining the pharynx. In childhood most of the contagious diseases, such as measles, scarlet fever, etc., are accompanied by a secondary pharyngitis.

Symptoms. - The disease is ushered in suddenly by profuse redness of the mucous membrane of the pharynx and a temperature which may reach $103^{\circ} \mathrm{F}$. to $104^{\circ} \mathrm{F}$. in a child. The whole vault of the pharynx, uvula, and fauces, may be involved. The inflammation pursues the same course as an inflammation of the mucous membrane anywhere. There is congestion, swelling, dryness, followed by an oversecretion of mucus, which soon becomes mucopurulent. The surrounding lymph-glands may be slightly enlarged. There is pain at the angle of the jaw and upon swallowing, a hacking cough, and stiffness and tenderness of the muscles of the neck. Fxtension to the larynx may cause hoarseness; to the ear, deafness (through the Eustachian tube). An inspection of the throat reveals a red and swollen mucous membrane. Simple, rheumatic, follicular, and infectious varieties are found. The first three explain themselves and the last is associated with the infectious fevers.

Treatment.- The local treatment is the same as in tonsillitis, with the addition of a steam spray medicated with compound tincture of benzoin, which frequently gives added relief. Pellets of ice may be used to good advantage.

All cases of acute sore throat should be looked upon with suspicion, as in most contagious diseases it is the first symptom. This is especially true of measles and diphtheria. If either of these diseases is prevalent, the case should be isolated until the time for the appear- 
ance of the rash has past or the culture from throat is found to be negative.

Uvulitis is an inflammation of the uvula or soft palate associated with pharyngitis. At times an elongation of the uvula renders it necessary to remove a small portion.

Chronic pharyngitis is not common in childhood. It usually results from repeated acute attacks and the improper use of the voice.

Symptoms.-The voice is husky and there is an increased secretion, so that there is a constant desire to clear the throat. Disagreeable sensations, such as fulness, tickling, and the like are frequently noted.

Four forms are found: the hypertrophic pharyngitis in which the membrane has become permanently thickened and causes a constant clearing of the throat.

An atrophic pharyngitis, in which the membrane is thinned out and secretion is lacking.

Ulcerative pharyngitis, in which the membrane is covered with ulcers due to simple inflammation, or the ulcers of syphilis or tuberculosis.

Phlegmonous pharyngitis, or retropharyngeal abscess, is due to a suppuration of the tissues behind the pharynx caused by caries of the cervical vertebræ, the impaction of a foreign body, or as a sequel to one of the infectious fevers.

The cases which arise independent of the above causes must be considered (Holt) as a retropharyngeal adenitis (inflammation of the lymph-glands). This may advance to the stage of suppuration, as it does in the majority of cases, when pus is present, or in very rare cases the inflammation may cease before this stage is reached. 
Children under one year of age are those most often attacked. It is rarely seen over five years of age.

The symptoms are as follows. At first the child may have severe nasal pharyngeal symptoms, accompanied by fever. These symptoms subside, but the fever still remains above normal. Several days later the temperature again rises to $103^{\circ} \mathrm{F}$. and the local and pressure-symptoms appear.

In other cases the onset is sudden and the local and pressure-symptoms are the first to manifest themselves.

The characteristic symptoms are due to pressure and are dyspnea from partial closure of the opening of the larynx, difficulty in swallowing from obstruction to the passage of food, mouth-breathing from the closure of the posterior nares, which gives rise to the characteristic "quack." The head is thrown back to elevate the mass and relieve the pressure. There are profound constitutional symptoms, the child appears desperately ill, and the appearance of the pharynx on inspection is characteristic. There is a bulging of one of the sides of the pharynx, the amount of protrusion varies. In some cases the uvula is pushed to one side and the pharynx filled up. The severity of the symptoms due to pressure depends upon the size of the protruding mass. The tumor soon fluctuates, showing the formation of pus, and the temperature becomes hectic (due to pus; high, irregular temperature, accompanied by chills, sweats, and pallor). If left to itself the abscess will rupture, usually in two or three days. Sometimes it is delayed for a week or two.

As soon as the fluctuation is discorered the abscess should be incised with a guarded bistoury. This is done so that the pus can be controlled. If allowed to rupture 
itself the pus may be swallowed or inspired. This may cause grave complications, such as aspiration pneumonia or asphyxia. The child should be firmly held in the nurse's lap, with head thrown back. A tongue depressor is used by the physician, as it can be immediately withdrawn, and not a mouth-gag. As soon as the abscess is opened the nurse should bend the child's head forward and thus allow the pus to run out of the mouth.

Some surgeons prefer to have the child lie on a table with head hanging backward over the end. After the abscess is lanced the head should be turned to one side to allow pus to escape. The after-treatment is simple, as the symptoms disappear as soon as the pus is removed.

Retropharyngeal abscess from Pott's disease is due to the softening of the cervical vertebræ with accumulation of pus behind the pharynx. It is slow in forming and not attended by much fever. Such abscesses are opened externally when the pressure-symptoms become of sufficient importance to demand relief. 
CHAPTER VI

\section{DISEASES OF THE DIGESTIVE TRACT}

(Continued)

\section{Diseases of THE Esophagus}

THE esophagus is rarely involved in inflammatory processes. The only condition of importance to a nurse is corrosive esophagitis and stricture.

Corrosion of the Esophagus.-This is almost always due to the swallowing of strong acids or alkalies. It causes intense pain and burning in the esophagus and swallowing is very painful.

Stricture is a secondary result of corrosion. The healing ulcer causes a narrowing of the alimentary canal at its location, and this interferes with swallowing.

Symptoms.-A slowly increasing difficulty in deglutition, with regurgitation of food. The esophagus is often much dilated above the stricture, and the food may collect in the pouch thus formed, so that regurgitation may be delayed for several hours. There is much loss of flesh. In bad cases of stricture it is necessary to place the child upon a liquid diet.

\section{Diseases of The Stomach}

In infancy we rarely find the stomach involved alone, being associated with the intestines in nearly all diseases. In older children the stomach conditions may be distinct.

Capacity.-At birth the capacity of the stomach is about $\mathrm{r} \frac{1}{5}$ ounce. 
For all practical purposes it is safe to say that the capacity of the stomach increases about I ounce for each month, up to eight months; then its development is slower. At one year the capacity is 9 ounces; at eighteen months I 2 ounces.

As the quantity of food taken at feeding increases, the time it takes the stomach to empty itself lengthens. From two to eight months of age it takes about two hours for mother's milk and two and a half hours for cows' milk.

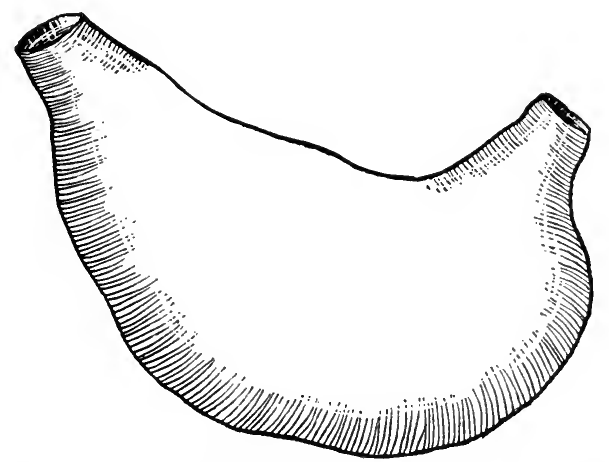

Fig. 37.-Stomach of infant at birth, natural size (J. P. C. Griffith).

The position at birth is peculiar in that it is almost vertical instead of being horizontal. This explains the ease with which newly born babes regurgitate. There is no attending discomfort, being like the running over of a filled bottle.

Digestion of Milk.-Mother's milk is coagulated into light, flocculent curds. Cows' milk is coagulated into tough, compact masses, and it takes about a half hour 
longer for the stomach to empty itself of this diet than it does of mother's milk.

Vomiting is a condition arising from a large number of sources. It may be watery or mucous; bilious or green, which occurs in any case where vomiting and straining are continued; bloody (hematemesis); or purulent, resulting from the rupture of an abscess into the stomach or esophagus.

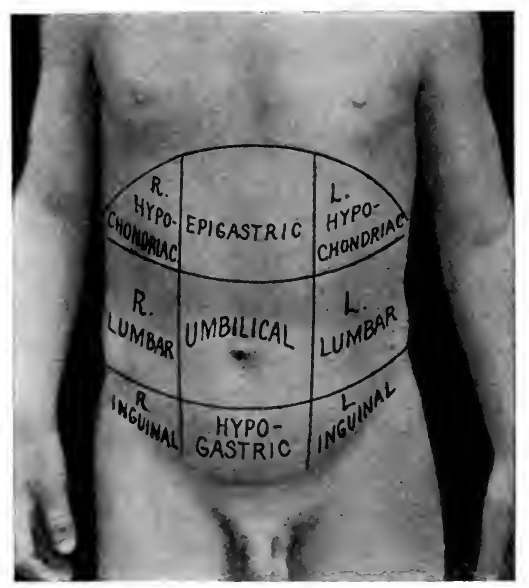

FiG. 38. - The abdominal regions. The heavy line at the upper border shows the extreme limit of the diaphragm. Imaginary lines divide the abdomen into different regions which, for the sake of clearness and precision, are known as the right and left hypochondriac, the epigastric, the right and left lumbar, the umbilical, the right and left inguinal or iliac, the hypogastric (Kerr).

Fecal vomit (stercoraceous) is indicative of intestinal obstruction and is recognized by its odor and appearance. Profuse vomiting, where large quantities of frothy, fermented material are ejected, is significant of gastric dilatation.

Vomiting without nausea, distress, or other gastric 
symptoms occurs in certain neuroses of the stomach, in hysteria, uremia, and in brain diseases, such as tumor or meningitis. This form of vomiting is seen at the onset of many fevers in childhood.

Habit vomiting is the name given to that form of emesis in which children vomit from habit alone, no disease of the stomach being present.

Cyclic vomiting is characterized by severe attacks of vomiting occurring at more or less regular intervals. It is uncommon and is of nervous origin.

Hematemesis is the term applied to vomiting of blood. If the hemorrhage is rapid and the blood immediately vomited, it may be bright red in color. However, it is usually retained for some time in the stomach before ejection, and is then dark brown in color, the so-called coffee-ground appearance. The blood is mixed with food and the subsequent stools are dark and tarry.

Hiccough or singultus results from a clonic spasm of the diaphragm. It is often noted as a temporary condition after eating and drinking. Persistent hiccough is frequently present in cases of extreme exhaustion following acute or chronic diseases.

Malformations and Malpositions of the Stomach.The cardiac and pyloric ends may be congenitally stenosed (no opening). At times the stomach is found in the thorax, gaining access through a rupture of the diaphragm.

\section{ACUTE GASTRIC INDIGESTION}

Acute gastric indigestion is the name applied to a series of symptoms caused by the inability of the stomach to properly digest.

The symptoms are vomiting, dulness, or excitement, 
and at times convulsions. The temperature ranges from $100^{\circ} \mathrm{F}$. to $102^{\circ} \mathrm{F}$., sometimes higher. 'The tongue is coated, the appetite lost, and the abdomen distended. In infants there is an associated diarrhea, the stools containing undigested food. From six to twelve hours after the onset the vomiting ceases and the symptoms disappear.

\section{GASTRITIS}

Acute Gastritis is an acute inflammation of the stomach. The mucous membrane is red, sticky, and lusterless; it is swollen and covered with thick mucus.

Symptoms.-They vary much in degree. In severe cases there is moderate fever $\left(102^{\circ} \mathrm{F}\right.$. to $103^{\circ} \mathrm{F}$.) and its associated phenomena. There is loss of appetite, a coated tongue, and intense pain in the epigastric region, which is tender to the touch. In addition there is persistent vomiting, thirst, and considerable prostration. Jaundice may follow from the extension of the catarrh to the bile-ducts (gastroduodenitis), and diarrhea from extension to the intestines.

The treatment is absolute rest. If the stomach is not entirely empty an emetic should be employed. To relieve the pain in the stomach local applications such as turpentine stupes or a mustard plaster will be found effective. In severe cases no food should be given by the mouth. To allay the thirst cracked ice may be given, and later milk and lime-water.

Chronic Gastritis (Chronic Gastric Indigestion, Dyspepsia).- This is a chronic indigestion and signifies a group of symptoms which accompany every disease of the stomach. When, however, the symptoms depend upon nothing more than simple atony, hypersensitiveness, 
or chronic catarrh, the condition is spoken of as a distinct affection. Corresponding to this view there are three forms recognized: (I) Atonic, (2) nervous, (3) catarrhal.

In infancy chronic gastritis is due to the abundant, tough, adherent mucus lining the stomach. This interferes with digestion, even though the stomach secretions are normal.

The symptoms are: long retention of food, vomiting six to eight hours after eating, signs of general malnutrition, and undigested food in stools. There is also dilatation of the stomach. In infants under three months the prognosis is bad.

In older children chronic gastritis is usually caused by gastric irritants such as tea and coffee in excess, by dietetic errors such as insufficient mastication from bad teeth, hurried eating, too much food, insufficient food, coarse or improperly cooked food, excessive dilution of food with liquids, excessive condiments, and irregular eating.

Symptoms of chronic gastritis are: coated tongue, perverted appetite, distress after eating, eructations, flatulence, heart-burn, palpitation, headache, vertigo, disturbed sleep, and lassitude.

In atonic dyspepsia the above symptoms are present and the pain usually appears some time after eating.

In nervous dyspepsia the above symptoms appear in nervous children. The symptoms vary greatly. At one time there will be anorexia, at another an inordinate appetite, and at still another a perverted taste. Pain and vomiting or retching occur just as frequently when the stomach is empty as when it is full.

In catarrhal dyspepsia a condition of chronic inflammation of the stomach exists. Just as in a chronic inflamma- 
tion of the mucous membrane in any other part of the body, so here there is a thickening of the membrane and the process of digestion is interfered with. The food remains for a long time in the stomach and undergoes fermentation; thus eructations of gas and sour liquids are frequent. 'There is more or less nausea, with vomiting, at all times, but especially so in the morning when the frothy mucus, which has collected over the mucous membrane during the night, is vomited together with much retained, fermented food.

In catarrhal dyspepsia the nurse is often instructed to wash the patient's stomach every morning by lavage (see page $4 \mathrm{I} 4$ ).

\section{GASTRALGIA}

Gastralgia is a painful, paroxysmal (intermittent) affection of the stomach not associated with any organic lesion.

Symptoms.- There are paroxysms of severe pain in the epigastrium, usually radiating to the back and occurring when the stomach is empty. It is relieved by pressure and the ingestion of foods or warm, stimulating drinks.

Treatment.-The child should be put to bed and hot water or turpentine stupes applied to the epigastrium. If the feet are cold apply hot-water bags there. Hot water containing five or ten drops of brandy and five drops of turpentine should be sipped.

\section{GASTRIC ULCER}

This is a rare condition in childhood. Ulcers may result from follicular gastritis, tuberculosis, or without obvious exciting cause. The latter is probably due to the digestion of a portion of the stomach by its own juices. This occurs when some local disturbance of the circulation 
shuts off the blood-supply to a portion of the stomach walls, the lowered vitality of that portion permitting the gastric juice to digest it. This produces the ulcer.

A gastric ulcer is round or oval and is usually situated at the pylorus on the posterior wall, near the lesser curvature. It is a punched-out ulcer, the apex toward the peritoneum, while the floor is usually formed by one of the coats of the stomach. A series of ulcers is not uncommon.

Symptoms. - The general symptoms of dyspepsia are present, and in addition the following characteristic symptoms: Pain, which may be severe, appears soon after eating and almost always radiates toward the back. Hemorrhage is present in one-half of all cases. The bleeding may be profuse and the blood bright red. Localized tenderness, nearly always two or three inches above the umbilicus. Vomiting, occurring an hour or two after eating and at the height of the pain. Hyperacidity, which is an increase in the hydrochloric acid after a test-meal.

This is a dangerous affection, demanding absolute rest in bed and rectal feeding.

\section{DILATATION OF THE STOMACH}

Moderate dilatation is often seen, a very marked dilatation is rare.

Causes.-Rickets, chronic gastritis, and pyloric stenosis.

The only symptoms present in most cases are those of chronic gastric indigestion.

In stenosis of the pylorus there is added vomiting of large quantities of fermented food, which occurs after the lapse of several hours.

In some cases of gastric dilatation the stomach is washed daily (see Lavage, page 4r4). 
Gastroptosis and enteroptosis is a prolapse or downward displacement of the stomach and intestines.

\section{TEST-MEALS}

The ordinary test-meal consists of a dry roll and twothirds of a pint of water or weak tea, without milk or sugar.

In testing for lactic acid the test-meal should consist of a tablespoonful of oatmeal to a liter of water, flavored with a small quantity of salt.

Method of Administration.- The child should be given a very light breakfast. Four hours later the stomachtube should be introduced and the stomach washed (see Lavage, page 4i4). The meal should then be eaten, and in an hour recovered by means of the stomach-tube. About 40 c. c. should be obtained.

\section{NURSING}

In diseases of the upper gastro-intestinal tract the room should be light and sunshiny, well ventilated, and kept at an even temperature.

The clothing should never bind the abdomen.

Bathing may be continued, except in the more severe forms of illness and in sore throat.

The food should be carefully prepared and given absolutely according to instructions. At times in severe vomiting it is necessary to prohibit food by the mouth. Nothing should then be allowed to enter the stomach.

The character of the vomit must be noted; the length of time after eating it occurs is important; and the presence of blood should be immediately reported.

Unless there is fever the temperature, pulse, and respirations need be taken but once or twice a day. 


\section{CHAPTER VII}

\section{DISEASES OF THE DIGESTIVE TRACT}

(Continued)

\section{DisEASES OF THE INTESTINES}

THE small intestine at birth is about 9 feet long, and the large intestine about 18 inches long. The lower half of this length is occupied by the sigmoid flexure.

Feces.--The first stools after birth are called meconium. Four to six stools a day of this discharge are natural. By the fifth day the stools should assume the appearance of milk feces.

Milk Feces.-The normal amount discharged by a healthy nursing infant is 2 or 3 ounces. They are soft, yellow, and of good consistency.

Mother's milk and cows' milk give practically the same stool. The number of stools during the first two weeks is from three to six daily. After the first month two stools a day is the average.

Symptoms.- The chief symptoms of any disease of the intestines are constipation, diarrhea, and tormina. When the rectum is the seat of the lesion we have added tenesmus.

Constipation is an unnatural retention of fecal matter. Its symptoms are infrequent stools, dyspepsia, fetid breath, headache, vertigo, lassitude, and anemia. In aggravated cases we frequently find hemorrhoids, fissures, fistulæ, and prolapse of the rectum accompanying these symptoms.

In infancy ordinary constipation nearly always can be corrected by the proper milk mixture, increase in the fats 
being all that is necessary. A soap-stick, some form of suppository, or introduction of the greased little finger act well in stubborn cases. At times enemas are necessary. These should not be used continuously.

In older children ordinary constipation can usually be overcome by a regular time for defecation and systematic exercise; abdominal massage and electricity are valuable aids. Encourage the use of water, bran-bread, green vegetables, and stewed fruit. A glass of water before breakfast is often all that is required.

Chronic Constipation.-As long as the child has the proper strength food for its age, constipation should not be troublesome. In artificially fed children, however, cases of chronic constipation are quite frequent. The nurse can correct this fault to a great extent by proper management of the case.

In older children the most important measure is to establish a regular time for stool. After breakfast is the best hour. The diet should be mixed, starchy food restricted, and fruits encouraged. Meat and green vegetables should be eaten at least once a day, oatmeal is the best cereal, and orange-juice and stewed prunes the best fruit. Massage should be practised twice a day, after retiring and in the morning. The proper method of giving massage is to use only the hand (without grease of any kind), rubbing the abdomen with a circular motion. The object is to move the skin and muscles upon the intestines, which starts peristalsis, the worm-like movements that force the feces along. Exercise is accomplished during playtime, and is usually sufficient. Suppositories are valuable at times to start the habit of defecation at a regular hour, but should not be continued longer than 
absolutely necessary. Gluten and glycerin suppositories are the best. If injections are necessary, I dr. of glycerin to $\frac{1}{2}$ ounce of water gives the most immediate results. At stool a low chair aids the child better than the high seat for adults.

Diarrhea is a condition in which the stools are either too frequent or too loose. Like dyspepsia, it is a symptom of many pathologic conditions. Any. condition which tends to lessen the peristalsis of the bowel will cause constipation; any condition which tends to irritate the mucous membrane of the bowel will usually cause diarrhea.

Tormina or intestinal colic is a painful, spasmodic affection of the intestines. It is generally the result of irritating food, flatulence, or fecal accumulation. It is characterized by paroxysms of severe pain of a twisting character centering around the umbilicus and relieved by pressure. The abdomen is usually distended. Severe attacks may lead to incipient collapse, indicated by cold sweats, pinched features, feeble pulse, and vomiting. The attacks often last from a few minutes to several hours, and generally end by a discharge of flatus. In severe attacks enemas, hot applications, aromatic spirits of ammonia, and paregoric are necessary.

Tenesmus is a feeling of fulness in the rectum with a constant desire to defecate.

\section{MALFORMATIONS}

Congenital atresia of the anus is seen occasionally. It should always be looked for after birth. Through some fault of nature in these cases the rectum becomes constricted, or the skin covers its outlet. This prevents any 
fecal passage. It results in death if prompt relief is not obtained.

A baby that does not have a bowel movement in the first twenty-four or forty-eight hours should be examined immediately.

\section{DIARRHEA}

Diarrhea is an acute inflammation of the mucous membrane lining the intestines. It is the so-called intestinal catarrh. The different varieties of acute diarrhea are: mechanical, caused by foods which act as foreign bodies; $d r u g$, caused by any of the ordinary cathartics in susceptible children; acute intestinal indigestion; nervous diarrhea; and diarrhea of certain diseases like uremia.

The character of the diarrhea depends upon the seat of the lesion. Inflammation high up in the bowel causes yellow and greenish stools; in the lower bowel more mucus and blood are found and less of the undigested food elements. The lower in the bowel the seat of the inflammation is, the more severe the symptoms become; the temperature is higher, the prostration greater, and the stools are mixed with blood. When the lower colon and rectum are involved, in addition, there is tenesmus, which is a sensation of fulness of the rectum, with a constant desire to defecate.

Acute Intestinal Indigestion.-In young children an acute attack of indigestion shows both gastric and intestinal symptoms; the intestinal symptoms, however, are always the more marked of the two. In older children intestinal indigestion alone is seen.

The symptoms are colicky pain, distention, and diarrhea.

The pain is localized in the stomach and around the umbilicus. About an hour or two after the onset of the 
attack diarrhea develops. From four to twelve stools are passed. They are greenish-yellow in infants, and contain undigested food. There is fever, $100^{\circ} \mathrm{F}$. to $102^{\circ} \mathrm{F}$. The pulse is rapid and the features pale and pinched.

Treatment.-Give castor oil and restrict the diet. In nursing babies give barley-water for twenty-four hours, and at the end of this time return to the breast. The feedings should be at six-hour intervals and the baby allowed to remain at the breast only for five minutes at a feeding. Barley-water is given in the interval. The diet of older children in the acute stage should be similar to that of an infant. Later, broths, eggs, milk, and dried bread or toast can be given. Fruit, vegetables, and cereals should be withheld for several days and then returned to slowly.

Chronic Intestinal Indigestion.-In infants the symptoms at times resemble marasmus. The symptoms most often seen are loss in weight, anemia, colicky pain, alternating diarrhea and constipation. The bowel movements are characteristic. If there is diarrhea the stools are greenish and often contain white curds. If constipated, the stools are often white. The child cries a great deal, is very restless, and sleeps poorly.

Treatment.-It is in these cases that the proper modification of milk does so much good. In very stubborn cases buttermilk, properly prepared, seems to cure.

The proper way to prepare buttermilk is as follows: Flour, $3^{\frac{3}{4}}$ dr.; cane sugar, I5 dr.; buttermilk, I quart. Bring up to the boiling-point, stirring continuously. Then cool rapidly. While the percentage of proteids in this mixture is much higher than is usually given in such conditions, it is more easily and rapidly digested. Often 
the improvement is remarkable. Buttermilk should not be used for any length of time without the addition of cream.

In older children, from four to seven years of age, the symptoms are as follows: 'The child is under-developed, pale, thin, and has a prominent abdomen. There are dark rings under the eyes, they easily tire, and are fretful and emotional. The stools are foul, there may be constipation or diarrhea, and a great deal of gas. Such children frequently grind their teeth, giving rise to the supposition that they have worms. Convulsions are not uncommon.

Treatment.- In such cases a trained nurse is invaluable. The chief thing to regulate is the diet. This should be placed entirely in her hands. It is a fact the the principal diet of children suffering with this condition usually consists of sugar, potatoes, and oatmeal. These should be interdicted, and for a beginning a diet of rare meat (scraped beefsteak or mutton), and milk instituted. Under the physician's orders, additions will be made, consisting of fruit, kumiss, stale bread, raw oysters, vegetables, etc. Potatoes and oatmeal should be forbidden for some time in these cases. Proper clothing, cold sponging in the morning, open-air exercise, and cool sleeping-rooms are of equal importance.

Summer Diarrhea.-When diarrhea attacks young children in the summer time, it is the so-called summer diarrhea of childhood. This is the most fatal disease of childhood. It occurs in epidemic form regularly every summer in most large cities. The changes in the bowel are slight, amounting in most cases only to a superficial catarrhal inflammation, often bearing no relation to the severity of the symptoms. These are mainly due to the 
absorption of toxic materials resulting from putrefactive changes in the stomach and intestines (Holt).

The chief cause of summer diarrhea is bad milk. The term "milk infection" is frequently used by physicians in defining this condition.

Milk which has been delivered to the consumer under the most favorable conditions will show a number of bacteria on examination.

When proper aseptic precautions are taken at a dairy, the herd is proved to be physically sound, the stables are clean and hygienic, the cattle's food is scientifically supervised, the milk cans and the clothing of the dairymen are sterilized, their hands and the udders of the cows are thoroughly cleansed, the milk is immediately placed in sealed glass quart bottles, packed in ice, and delivered as quickly as possible, still from two to ten thousand bacteria per cubic centimeter will be present. One can imagine the number of bacteria in a cubic centimeter of the ordinary milk which is delivered by the itinerant milk man. A hundred thousand per cubic centimeter is common in the summer time, and in samples of bad milk over a million have been found.

The Philadelphia Pediatric Society, which has the supervision of issuing certified milk certificates in this vicinity, has made ten thousand bacteria to the cubic centimeter the limit for certified milk. As there are only about a half dozen dairies which can comply with this standard in an area which comprises about 2,000,000 people, one can see the difficulty in obtaining good milk.

When such milk is given to an infant the toxins generated in its digestive tract produces the symptoms so characteristic of this condition. 
The symptoms of the milder form are frequent stools, three to twelve a day. They are of yellowish or greenish color and contain undigested food. They are colicky pains with rumbling noises (borborygmi), and slight fever with its attending phenomena These symptoms usually follow an acute attack of indigestion and are accompanied by gastric symptoms which mayset in at almost any time after its onset, the principal feature of which is the persistent vomiting. After a time the stools become offensive, mucus is present, the appetite may be normal, but is often impaired and may be almost lost. The tongue is coated, the mucous membrane of the mouth is congested, and in very young infants is often covered with thrush. The general health may not be noticeably affected for several days, but more often the infants become pale, their limbs grow soft and flabby, they lose their spirits, are fretful, sleep badly, and the scales show a decrease in weight of from one to two pounds a week. Relapses are common, especially if the infants are placed upon a milk diet too soon, or by overfeeding.

In the more severe form the attack may begin abruptly, or there may have been symptoms of the milder variety for several days. The temperature rises rapidly to $103^{\circ} \mathrm{F}$., often to $105^{\circ} \mathrm{F}$. There is great thirst. The children are restless, excited, and may have convulsions, or they may be just the opposite, and lie in a dull stupor. From four to six hours after the onset, vomiting begins, milk appearing as tough curds and very sour. After the stomach is emptied retching continues, and everything given by the mouth is almost immediately rejected. Diarrhea follows. The stools are thin, yellowish, greenish, brownish, or mixed, very offensive, and frequently accompanied by the discharge 
of large quantities of gas with accompanying colicky pains. In fact, the foul odor, the colic, and the discharge of flatus is the most characteristic symptom of this form of diarrhea. From five to twenty-five fluid stools in twenty-four hours, frequently of good size, and very offensive, are seen after the first day. In a few days mucus may appear. After two or three days there is generally a reaction and the child improves. The stools, however, may continue loose for five or six days, gradually assuming their normal character.

If there is no reaction, steadily increasing prostration, continued high temperature, and diarrhea may cause a fatal termination of the case.

In other cases the symptoms merge into those of enterocolitis. At times there may be a series of acute attacks a week or ten days apart; in the interval all symptoms are absent except that the stools never become normal. The third or fourth such attack may merge into enterocolitis.

Prophylaxis.-As this disease is caused by impure milk, all that is said about the care of the milk in the house and the sterilization of bottles and nipples on page 366 should be carefully read. It is frequently necessary for all milk to be pasteurized during the summer months (see page 335).

During the hot months the infant's clothing should be light flannel; a single-piece dress is the best. Their napkins should be changed immediately after soiling. They should have fresh air, sunlight, and frequent bathings. Maternal nursing should be practised in every case where it is possible. Weaning should be avoided during the summer months. Overfeeding should be prohibited. Less food at a feeding by one-third, and more water, is a good rule to follow during the hot weather. Early 
attention should be given to all mild disorders of the gastro-intestinal tract. Finally, if artificial feeding is nècessary, the proper modification of cows' milk should be used (see Chapter XVIII).

Nursing.-If possible, children suffering from summer diarrhea should be sent to the seashore. 'They should not be allowed to walk, but should lie out in the fresh air as much as possible.

Fresh air, quiet, proper clothing, and frequent bathing are essential. All soiled diapers should be immediately changed, the buttocks carefully washed, and the movements disinfected as described on page 436 . The character and frequency of the stools must be reported. It is of the utmost importance to stop the milk, as in the early part of the attack digestion is arrested. Small quantities of cold whey, barley-, or albumin-water should be frequently given. If all food is rejected or vomited, the best results are obtained from giving the stomach absolute rest.

Maternal nursing should be withheld until twenty-four hours after the vomiting has ceased (which is usually within twelve hours). 'Then the physician may order a tentative return to the breast. The interval between nursings should then be four hours, and only one-quarter of the usual quantity allowed. This may be regulated by allowing the infant to nurse only for two or three minutes at first. Between nursings whey, barley-, and albuminwater may be given, so that the infant takes something every two hours. If the indications permit, the breastfeeding will be gradually increased.

When the child is artificially fed, cow's' milk is absolutely withheld during the stage of acute symptoms, and for 
several days after. When it is begun the physician may have to use various methods to render it digestible. The methods for carrying out any special instruction in this line, such as peptonizing, etc., will be found in Chapter XVIII. During the period when milk is suspended he will use such substitutes for milk as rice- or barley-water, wine-whey, malted soups and foods, albumin-water, fresh beef-juice, animal broths, liquid peptonoids, condensed milk, etc. The methods for preparing these foods will be found in Chapter XVII.

In older children the food is withheld until vomiting ceases, and then broths and beef-juices given. Later, thin gruels made with milk, koumiss, and such foods are substituted. Solid foods should not be allowed for several days after the stools are normal.

A proper acquaintance with the appearance and taste of every food ordered is essential, and a careful record of the amounts taken must be kept. At times the physician will order stimulants placed in the foods.

No cases do worse than those in which the mother or nurse in charge cannot be made to appreciate the value of starvation, but insist upon giving food, especially milk, in violation of the rules laid down.

Lavage and irrigation of the colon are essential adjuncts to the medicinal treatment, and the nurse must be prepared to apply these measures when ordered (see pages $4 \mathrm{I} 4$ and 4I 7 ).

The graduated cold bath will be ordered at times by the physician (see page 396).

At the onset of an attack of diarrhea in summer, give a dose of castor oil and starve the child for twenty-four hours. Barley-water may be used to allay the thirst. 


\section{ENTEROCOLITIS, ILEOCOLITIS, OR DYSENTERY}

Of this disease there is a catarrhal, an ulcerative, a membranous, and a chronic form. The severity of the symptoms is greater in the ulcerative and membranous forms than in the catarrhal.

Enterocolitis may follow one of the intestinal conditions previously discussed, or it may be the initial disease.

Its distinguishing symptoms are mucus and blood in the stools, colicky pains, and tenesmus. Often the amount of mucu's is quite large, at times the entire movement may be composed of it. The characteristic appearance of mucus is a grayish or whitish jelly-like mass, often streaked through the stool. Blood rarely appears as clots, but is also streaked through the movement. 'The stools are usually' small in size, odorless, and accompanied by a great deal of straining, often only a teaspoonful is passed at one time. After the first acute symptoms are over, the blood usually disappears, but the mucus is persistent, and in all cases the recovery is protracted. Relapses are common, and even after the child has fully recovered subsequent attacks are easily excited by errors in diet. This is an important point to keep in mind, as great care must be exercised in supervising the diet of such children; very slight indescretions have produced fatal recurrences of the disease. Impress upon the family the importance of keeping strictly to the physician's orders.

A very common complication of enterocolitis is bronchopneumonia, which usually produces a fatal result.

Everything that was said in reference to the cause of summer diarrhea applies to enterocolitis.

In the acute catarrhal form the mucous membrane 
of the colon is red, swollen, edematous, and in some cases ulcerated.

Symptoms.-The onset is sudden. There are frequent stools, at first yellow, later green and mixed with curds, mucus, and blood, and sometimes material resembling chopped spinach. Temperature ranges from IO2 ${ }^{\circ} \mathrm{F}$. to $103^{\circ} \mathrm{F}$. The abdomen is distended and tender along the colon. Vomiting is rarely persistent. In the milder cases the mucus and blood continue to appear in the stools for from four to five days. The diarrhea continues for one or two weeks.

In the ulcerative form the mucous membrane is swollen from edema and cellular infiltration. The latter causes superficial necrosis and formation of irregular ulcers which more or less undermine the surrounding mucosa.

The symptoms of ulcerative enterocolitis are similar to the simple catarrhal form, but the disease is more protracted and often marked by intermissions and exacerbations. The stools are more fluid and the mucus and blood persist.

In membranous enterocolitis the mucous membrane is intensely swollen and covered by a false membrane. The separation of the membrane is followed by ulceration and sloughing.

The symptoms are the general symptoms of dysentery plus those of the typhoid condition. The stools also contain false membrane and sloughs. The child grows pale, wastes, and assumes a senile appearance. Death may be preceded by coma and convulsions.

The prognosis is always grave in membranous enterocolitis, yet recoveries do take place under favorable conditions. 
Nursing of Enterocolitis.-What was said under summer diarrhea holds good in ileocolitis. In older children there must be absolute rest, enforced use of the bed-pan, and the proper restriction of the diet. The milk must be stopped immediately and in no instance given without the physician's orders. For the pain apply externally hot fomentations and mustard poultices. Small pellets of ice introduced into the rectum every two or three minutes for half an hour, or cold compresses applied externally, will frequently relieve the tenesmus. In severe cases of tenesmus the physician may order thin starchwater injections containing to to 20 drops of laudanum to the pint. Irrigation is often practised.

In diseases of the intestines the room should be light, cheerful, and well ventilated. Bathing need not be dispensed with unless the child is too sick to stand it.

A woolen binder worn around the abdomen is often of use. Cold feet should be avoided in winter, and overdressing in summer is harmful.

The feedings should be carefully prepared and the amount taken accurately recorded; the character of the stools reported, and the bowel movements covered with carbolic acid, I: 20 (see page 269).

The temperature, pulse, and respirations should be taken at least twice a day.

\section{CHOLERA INFANTUM}

This is an acute disease of childhood characterized by high fever, vomiting, purging, and collapse.

It is now generally taught that the severe symptoms of this disease are produced by the result of a toxemia or poisoning of the system. The poison is produced in the 
intestinal tract and absorbed. It receives its name from the similarity of the symptoms, in well-marked cases, to Asiatic cholera.

In the majority of cases the disease attacks children who have been suffering from some form of intestinal trouble. At times children who have been perfectly well are stricken.

Symptoms.-The onset may be gradual or abrupt. Diarrhea is usually the initial symptom. The stools are thin and serous or watery, and have a musty odor. Vomiting soon develops, and the irritability is so great that everything is rejected. The thirst is intense, and the temperature is very high, $105^{\circ} \mathrm{F}$. to $108^{\circ} \mathrm{F}$; t the pulse is rapid and feeble and the urine scanty. Collapse follows, and is indicated by the pinched features, hollow eyes, sunken fontanels, and the cold surface of the body. Even at this time reaction may set in, but, more commonly, death results from exhaustion.

Treatment.-Prophylaxis. - What has been said concerning prophylaxis, under previous diseases of the intestinal tract, should be practised to avoid such a form of the disease as this.

Nursing.-As everything swallowed during an attack only aggravates the vomiting, nothing should be given by the mouth except ice and iced champagne.

The physician may find it necessary to wash out the stomach (Lavage, page 4I4) and irrigate the bowel (page 4i 7 ).

At times hypodermoclysis (see page 424 ) is resorted to. The serous or watery diarrhea so depletes the fluids of the body that this is necessary.

The cold bath or tubbing is used to counteract the fever (page 396). 
In collapse give a mustard tub, $110^{\circ} \mathrm{F}$, then place the child in a horizontal position, cover with warm blankets, and administer stimulants freely.

Cholera morbus is a term given to a disease, similar to cholera infantum, in older children. The symptoms, however, are not so severe. This disease is seen in the summer season, caused by eating unripe fruit, and sudden changes of temperature, such as bathing and swimming.

Symptoms.-Intense cramps in the stomach, vomiting, and purging of bilious material, moderate fever, and great prostration. In severe cases the discharge becomes serous, and symptoms of collapse develop.

\section{TUBERCULOSIS OF THE INTESTINES}

The symptoms closely resemble chronic ileocolitis. Ulceration occurs and the tubercle bacillus is found in the stools. It accompanies general tuberculosis of the body.

\section{APPENDICITIS}

This is an inflammation of the appendix vermiformis. It is a medical condition until an operation is demanded.

There are three varieties: catarrhal, ulcerative, and interstitial. In mild cases the appearance of the appendix is similar to that of catarrhal inflammation of a mucous membrane elsewhere. In severe forms the appendix is infiltrated with round cells and the mucous membrane is denuded of its epithelium and presents a granular appearance. This latter form may eventuate in septic peritonitis, chronic appendicitis with relapses (recurrent appendicitis), or union of the granulating surfaces with complete obliteration (appendicitis obliterans).

In ulcerative appendicitis the wall of the appendix 
is the seat of a more or less localized ulcer. It may be associated with the presence of a fecal concretion or a foreign body, or it may be the result of a typhoid or tubercular infection.

In interstitial appendicitis the wall of the appendix is the seat of a necrosis which is not infrequently gangrenous. It terminates by perforation, thereby exciting the most virulent type of peritonitis.

The chief causes of appendicitis are exposure, errors in diet, intestinal catarrh with extension to the appendix, traumatism, lodgment in the appendix of fecal concretions or foreign bodies. It may follow some infectious disease, as typhoid, influenza, or tuberculosis. It may be induced by the twisting of the appendix. Any of these conditions will interfere with the blood-supply of the appendix, and this is the real reason for the inflammation.

Symptoms.--Sudden pain, often general at first, but later most marked in the right iliac fossa. Localized tenderness, most frequently detected over McBurney's point, which is the center of the line drawn between the anterior superior spinous process of the ilium (the anterior prominence on the pelvic bone), and the umbilicus. Fever $103^{\circ} \mathrm{F}$. to $104^{\circ} \mathrm{F}$. There is a localized rigidity in the right iliac fossa and the presence of a definite tumor. Dorsal decubitus with right thigh flexed. Gastro-intestinal disturbances, such as anorexia, nausea, vomiting, constipation, or rarely, diarrhea.

Terminations: resolution, general peritonitis, localized abscess.

Treatment.-Absolute rest and liquid diet. An ice cap should be kept constantly over McBurney's point. Move the bowel only by enema. An operation is necessary at 
once in cases beginning suddenly with great severity, in ordinary cases where no improvement is noted after the lapse of forty-eight hours, when at any time there should be a sudden increase in the pain or a rapid diffusion of tenderness, and whenever a well-defined tumor can be detected in the right iliac fossa.

Nursing.-Previous to operating, spreading tenderness, vomiting, rise or fall in the temperature, and rapidity of the pulse must be reported immediately.

If ice-bags are ordered to be applied to McBurney's point, be sure they remain there. If vomiting occurs, the nurse should hold the sides of the abdomen to give as much relief as possible.

The room should be well ventilated and kept at an even temperature. Bathing should be dispensed with. Alcohol sponging will relieve discomfort.

After the operation the child should be kept flat on the back. The dressing should be carefully watched. Absolutely nothing should be given by the mouth without a physician's orders. The temperature, pulse, and respiration should be taken every three hours throughout the attack.

In septic cases the surgeon may order the child to be placed in a semireclining or sitting position after operation for purposes of drainage.

This can be maintained by a bed-rest and some contrivance to keep the child from slipping. A simple plan of procedure under these conditions is to take the seat of a swing, bore four holes, one at each corner, through which are passed the ends of four lengths of rope, which are securely knotted on the under side. After the desired 
position of the seat on the bed has been determined, the two lower ropes are tied to the head of the bed at the level of the mattress. The seat is then tilted to the proper angle, and the two upper ropes are tied to the top of the back of the bed.

The seat is then covered with a pillow upon which rests the child's buttocks. His legs are flexed over the edge of the seat, and his feet rest on a hot-water bag. This apparatus gives them a feeling of security and the angle of the seat may easily be changed by loosening or tightening the upper ropes.

Continuous saline irrigation of the bowel may be ordered (for method, see page $4 \mathrm{r} 8$ ).

\section{INTESTINAL OBSTRUCTION}

Intestinal obstruction is a condition in which the bowel becomes closed, preventing the passage of fecal material.

Causes.-Acute causes are congenital occlusion, intussusception, internal or external strangulation, and twists.

Chronic obstructions are: stricture from healing ulcer; unnatural accumulations, as of fecal masses; foreign bodies; gallstones; and tumors pressing from within or without.

Congenital occlusion is usually located at the anus or rectum (see page I29).

Intussusception is the slipping of a portion of the intestines into another portion immediately below it. This usually occurs at the ileocecal valve, the small intestines slipping into the large. This is due to unequal muscular contraction and an elongation of the mesentery (the attachment of the intestines to the abdominal wall). The invagination cuts off the blood-supply, and gangrene 
occurs. Unless promptly reduced, death occurs from this cause.

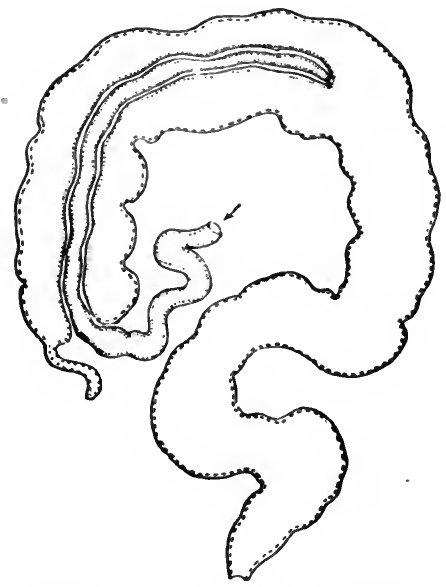

F16. 39.-Schematic representation of an ileocecal invagination. The mucous membrane is indicated by the dotted line (Frühwald and Westcott).

Symptoms.-The onset is often sudden. There may be a few loose stools, composed mostly of blood and mucus. At first there is paroxysmal pain in the side. Later this becomes continuous. Vomiting almost immediately sets in and is at first bilious, but after the obstruction is complete, it becomes fecal (stercoraceous). It is persistent, and everything that enters the stomach is immediately rejected. At first the abdomen is retracted, but it soon becomes distended, and the presence of $\mathrm{a}$ sausage-shaped tumor may be detected at the site of the intussusception. Prostration is marked. The tempera- 
ture ranges between $103^{\circ} \mathrm{F}$. to $104^{\circ} \mathrm{F}$. If gangrene occurs, the child dies of peritonitis.

Treatment.-High rectal enemas of large quantities of water or salt solution at times forces the bowel back and reduces the intussusception. In giving such enemas the buttocks should be well raised and the bottom of the bag four feet above the table. If this does not accomplish the result, an immediate operation is demanded.

Strangulation.-This occurs where there is a hernia or rupture. It may occur externally where the bowel slips through the abdominal ring, or internally if a portion of the gut slips through an opening in the diaphragm or under an adhesion. All of these conditions become strangulations where for any reason the bowel is compressed and the passage of feces stopped.

Twists or volvulus are found in the sigmoid flexure. They are true twists of the bowel, due to a relaxed mesentery.

Symptoms of acute obstruction are sudden and at first consist of paroxysmal pains, later becoming continuous. There is constipation; vomiting, persistent and becoming stercoraceous; abdominal distention; and collapse.

Treatment.-An immediate operation is imperative.

Chronic Obstructions.-Healing ulcers form scars which contract and act as a band constricting the intestines. Unnatural fecal masses, foreign bodies, and gallstones may obstruct by their bulk. Tumors may press upon the intestines, in this way compressing them, or tumors growing within the intestines may obstruct by their bulk (uncommon in childhood).

The symptoms of chronic intestinal obstructions develop slowly and are the same as those found in the acute form. 


\section{ANIMAL PARASITES}

Animal parasites or intestinal worms are tape-worms (tænia solium and tænia saginata), round worms (ascaris lumbricoides), and seat worms (oxyuris vermicularis.)

Tape-worms are formed of white segments. They are flat and may be from ten to fifteen feet in length. They gain entrance to the intestinal canal through uncooked beef or pork. The tania saginata or beef-worm is the most common in children.

Symptoms.-Various nervous symptoms exist. Anemia, if the worm is not expelled, and at times an inordinate appetite. Segments are seen in the stools.

Treatment.- The thorough cooking of all meats. If segments are discovered the child should be treated as follows: a light supper, and the following morning a saline purge, without any breakfast. After the purge has acted give olcoresin of male fern, I5 minims, in capsule, one every hour for four hours. Fifteen minutes after the last dose give castor oil. Milk should be the diet for the rest of the day.

It is absolutely necessary that the head of the worm should be recovered, for even though all the body is passed, if the head remain the worm will grow again. The subsequent stools should be closely watched. The head is very much smaller than the body and is attached to the neck, which gradually grows smaller as the head is approached. At the time of the expected passage of the worm it is well to half fill the receptacle with water. This permits the worm to float and avoids the breaking from its own weight. To recover the head a piece of muslin or fine mesh gauze should be stretched over a vessel and the movement poured into this. 
Ascaris Lumbricoides (Round-worms).- These worms are from five to ten inches in length and about as thick as a slate pencil. They are grayish-pink in color and are pointed at both ends. Usually six to a dozen are present in the bowel.

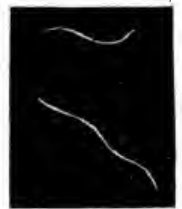

Fig. 40.-Oxyuris vermicularis, natural size (Kerr).
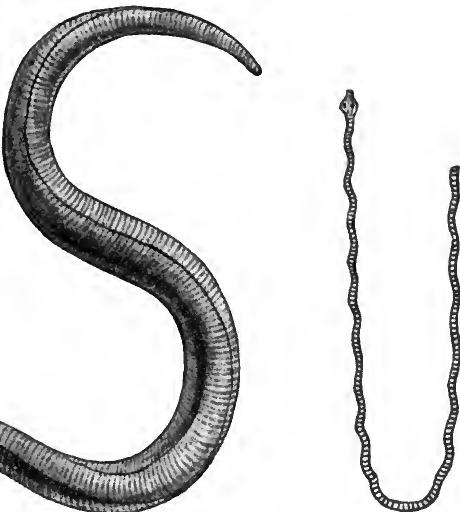

$a$

FIG. 42.-Teniæ: (a) Head and first segments; $(b)$ middle segments (Kerr).

Fig. 4r.-Ascaris lumbricoides (Kerr).

The symptoms are variable, nervous symptoms being the most marked. The worms are often found in the stools. They have a tendency to migrate, and are at times vomited.

Treatment.-Santonin, gr. 3 , to a child of five, given in three doses at four-hour intervals. The same plan of treatment as described under tape-worms should be followed, with the exception of santonin being used instead of male fern.

Oxyuris Vermicularis (Seat-wo rms).- These are small thread-worms about one-third inch in length. They occur in great numbers and inhabit the colon and rectum. 
Symptoms.-Intense itching of anus, especially at night, and the appearance of the worms at anus.

Treatment.-The use of bichlorid of mercury, I to Io,ooo after stool. Wiping the parts thoroughly and the injection of bichlorid of mercury, I to 10,000 , into the rectum will cure mild cases. Infusion of quassia is also used. Some cases are very troublesome. Blue ointment will relieve the itching.

Uncinaria Duodenalis (Hook-worms).-These are small thread-like worms, about three-quarters of an inch long, which attach themselves by means of four teeth to the mucous membrane of the small intestines, especially in the jejunum.

The loss of blood due to thousands of these worms being "hooked" to the lining of the intestines causes a severe and at times a fatal anemia. It is one of the most dangerous parasites met with in the human body. It is found in Egypt, Germany, Italy, Belgium, Switzerland, the West Indies (Jamaica), and through the southern portion of the United States.

The children affected become dull, listless, emaciated, and profoundly anemic.

The ova and embryos are contained in the stools, and are disseminated through dirt.

Treatment and Nursing.-Thymol is given to destroy the worms. Following its administration castor oil will usually expel large numbers of dead worms. The stools should be thoroughly disinfected (see page 436).

\section{DISEASES OF THE RECTUM}

Prolapse of Rectum.-This may be partial or complete. A simple eversion of the mucous membrane or a protrusion of three or four inches of the bowel. It occurs at stool. 
Treatment.-This can usually be replaced by gentle pressure with oiled fingers. Cold water will reduce congestion and assist in an obstinate reduction. To prevent recurrence have the child defecate upon its back, holding buttocks close together. In the more severe forms surgical treatment is demanded.

Fissure of the anus is a small linear ulcer of the mucous membrane covering the sphincter. It is very annoying and irritating.

Treatment.-Cleanliness and touching the ulcer with a silver stick will promptly cure.

Ischiorectal abscess is an abscess in the tissues surrounding the rectum.

Hemorrhoids or piles are the engorgement of the veins of the rectum. They may be internal or external. They give rise to pain at stool, and are not common in children. Cold-water injections give relief.

Incontinence of feces is the name given to the inability to control the evacuations.

Proctitis.-This is an inflammation of the rectum. When it occurs alone it is caused by the use of suppositories, injections, and seat-worms.

The principal symptoms are the passage of mucous and bloody stools with tenesmus.

Nursing. - The tenesmus is relieved by the introduction of pellets of ice into the rectum, or by means of a simpler method which is probably just as effective-the application of cold compresses to the anus.

Do not take the temperature by way of the rectum if it is diseased.

Give rectal irrigations slowly to avoid pain. 


\section{Diseases OF THE Liver}

Jaundice is a pigmentation of the skin and tissues with bile-pigment. It is caused by an obstruction to the flow of bile from the liver, through the common bile-duct, into the intestines. This obstruction in children is usually due to congestion or inflammation of the common bile-duct, the process being an extension from the small intestines. This obstruction prevents the bile from passing through its natural channels, so that it is backed up and thrown into the circulatory system and carried to all parts of the body.

The symptoms of jaundice are the yellow hue to the skin and conjunctiva of the eye; the latter is distinctive. The urine is dark and the stools are light.

Icterus neonatorum is jaundice of the newborn infant.

Catarrhal jaundice is the name applied to an acute catarrhal inflammation of the common bile-duct usually caused by extension from the bowel, characterized by the symptoms of gastro-enteritis plus jaundice.

Gallstones are concretions formed in the gall-bladder and composed for the most part of bile elements. These stones may lie latent, pass out along the common bile-duct, causing biliary colic, or may become impacted in the duct, giving rise to grave symptoms which demand an operation. This is not common in childhood.

Symptoms of biliary colic are sudden and intense pain over the liver, radiating to the right shoulder. This usually occurs an hour or two after eating. A chill with fever may mark the onset. Jaundice may be present from obstruction and the stone may be found in the stool.

Cholecystitis is inflammation of the gall-bladder.

Congestion of the liver may be active or passive, just as in the lungs. Active from arterial blood (too much 
blood from heart to the liver); passive from venous blood (obstruction to return of blood to heart).

Cirrhosis of the liver is a chronic disease characterized by an overgrowth of connective tissue and a destruction of the liver-cells.

There are two varieties, an atrophic form in which there is general contraction of the connective tissue in the liver and the organ becomes very much reduced in size.

A hypertrophic form in which there is no contraction of the connective tissue, but since the connective tissue is largely increased the liver actually enlarges.

Abscess and cysts of the liver are seen.

\section{Peritonitis}

This is an inflammation of the peritoneum. It may be primary, or secondary to some inflammation of the surrounding parts. It may be localized or general. It may have a serofibrinous, fibrinous, or purulent exudate. It may be acute or chronic.

Acute peritonitis is caused by exposure to cold and wet, traumatism or injury, and by extension of the inflammation from some adjacent structure, such as typhoid ulcer or appendicitis. It may be secondary to some general disease such as scarlet fever, rheumatism, or tuberculosis.

Symptoms.-The disease starts with a chill and fever, $102^{\circ} \mathrm{F}$. to $103^{\circ} \mathrm{F}$. There is a rapid, wiry pulse, abdominal pain, and tenderness so intense that abdominal respirations and body movements are inhibited. The child lies on its back with the thighs flexed, the features are pinched, the vomiting is persistent, the bowels are constipated. Hiccough is a common and troublesome symptom, the abdomen is greatly distended and is hard 
and board-like. In the large majority of cases death occurs in a few days.

Nursing.--The room should be kept at an even temperature; the bed-clothes should be supported by a frame over the abdomen.

The child should be carefully watched; any movement will cause excruciating pain. Bathing should be stopped. Champagne is often the only substance which will be retained. Cracked ice will alleviate the thirst. Drugs are often administered hypodermically.

If the child vomits, hold the sides of the abdomen to give as much relief as possible.

The temperature, pulse, and respirations should be taken every three hours.

Chronic peritonitis may occur unassociated with tuberculosis. It is usually a localized peritonitis; if general, it is associated with fluid in the abdomen.

Tuhercular Peritonitis. - The large majority of cases of chronic peritonitis are tubercular in character. It is usually associated with general tuberculosis or due to tuberculosis of the mesenteric lymph-glands.

Symptoms. - The abdomen usually contains fluid and there is an evening rise of temperature; emaciation and weakness are marked. The pain is not so severe as in the acute form of peritonitis, but there is diffuse tenderness. There are evidences of tuberculosis in the other organs.

Treatment.-It is in these cases that opening the abdomen is of such value.

\section{ASCITES}

Ascites is a dropsical condition of the peritoneum in which there is a serous effusion into the peritoneal cavity, usually a part of general dropsy. 


\section{CHAPTER VIII}

\section{DISEASES OF THE CIRCULATORY SYSTEM}

Anatomy.-The heart is a hollow, conic muscle normally situated in the left chest.

It is divided by muscular and fibrinous partitions into four cavities. The two upper chambers are called the right and left auricles, and the two lower, the right and left ventricles.

The right auricle is connected by an opening with the right ventricle called the tricuspid orifice. This passage is closed by the tricuspid valve, so-called because it has three leaflets.

The right auricle contains the orifice of the superior and inferior vena cava. This opening is surrounded by a sphincteric muscular band to prevent a back-flow of the blood.

The left auricle opens into the left ventricle by the mitral orifice, which is closed by the mitral valve, so called because it resembles a bishop's miter. The function of the valve is to prevent a return flow of blood to the chamber it has left.

The left auricle contains the orifice of the pulmonary vein.

The right ventricle contains the orifice of the pulmonary artery. This is closed by the pulmonary semilunar valves. 
The left ventricle contains the orifice of the aorta, closed by the aortic semilunar valves.

The valves are made of thin fibrous tissue and covered by the endocardium. They are attached to the heart-muscle walls by the corda tendina, little filaments of muscular tissue. Normally, the valves perfectly close

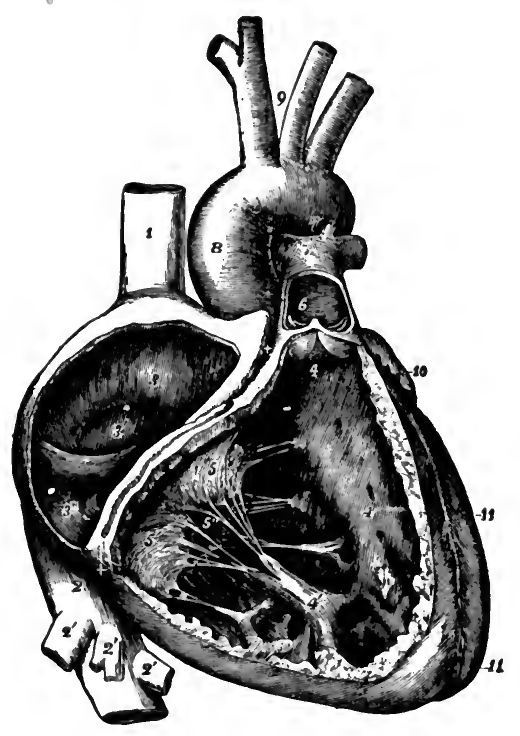

FIG. 43.-Right auricle and ventricle opened: 1, Superior vena cava; 2, inferior vena cava; 3 , right auricle; 4 , cavity of right ventricle; $4^{\prime}$, papillary muscles; $5^{\prime}, 5^{\prime \prime}$, $5^{\prime \prime \prime}$, tricuspid valve; 6 , pulmonary artery and semilunar valve; 7,8 , aorta; 10, left auricle; II, left ventricle. (Allen Thomson).

the orifice at which they are situated after the column of blood passes that point.

The endocardium is the lining membrane of the heart. It covers the inner walls of all the cavities and both sides of the leaflets of the valves. 
The pericardium is a serous membrane and forms a sack in which the heart is contained. It is like the pleura and covers the heart in a similar manner as that membrane does the lungs. One portion covers the heart-muscle intimately, being firmly attached to it; and the other portion is reflected, forming a closed sack. Between the two layers there is about one ounce of a serous fluid which lubricates the opposing surfaces.

The broad upper portion of the heart is called the base and the lower pointed portion, the apex.

The heart beats rhythmically from an inherent prop erty of its muscle and from nervous control. The pneumogastric nerve carries the principal fibers to it.

When the heart-beat is heard or felt the ventricles empty. This is called systole. The auricles empty between beats. This is called diastole.

The Circulation.- The blood enters the right auricle from the superior and inferior vena cava. It then passes through the tricuspid orifice to the right ventricle. From the right ventricle the course is through the pulmonary artery (the only artery in the body carrying venous blood) to the lungs, where it is purified and changed into arterial blood. It returns to the left auricle through the pulmonary vein, the only vein carrying arterial blood. From the right auricle the blood passes through the mitral orifice to the left ventricle and is then pumped into the aorta. The aorta supplies the general arterial circulation, distributing the blood through its divisions and subdivisions to all parts of the body. The arteries become smaller and smaller, until they become capillaries. Here the arterial blood is changed into venous blood. The venous blood is collected from the capillaries, emptied into veins, which 
become larger and larger, and finally flows into the superior vena cava (the head, neck, and upper extremities) or into the inferior vena cava (the trunk and lower extremities). The vena cava empties into the right auricle. The time taken for a complete circulation of a drop of blood in a newly-born child is twelve seconds; at three months, fifteen seconds; in the adult, twenty-two seconds (Vierordt).

The Fetal Circulation.-This is the circulation of the blood in the fetus. It differs from circulation after birth in that the blood is purified in the placenta and not in the lungs of the child. The placenta is attached to the walls of the womb and is expelled at birth, being called the after-birth.

Before birth the right and left auricles are connected by an opening called the foramen ovale. A large portion of the blood passes through this foramen directly into the left auricle. A smaller quantity passes through the tricuspid valve into the right ventricle.

At birth the umbilical cord is ligated and breathing is instituted in the child. The blood then ceases its flow to the placenta and the pulmonary circulation becomes of great importance. The circulation from this moment is the same as in after life. The passage through the foramen ovale and the ductus arteriosus becomes unnecessary, and, normally, these two openings are closed. The foramen ovale is almost closed at birth and entirely closed within six months after delivery. The ductus arteriosus closes in about ten days.

At times these openings remain patulous, they fail to close, and the fetal circulation persists after birth. This allows the venous blood in the right side of the heart to enter the arterial system, giving rise to what are known as 
blue babies. Before birth the sharp distinction between venous and arterial blood does not exist in the heart.

Malformations.-The patulous ductus arteriosus and foramen ovale constitute a deformity of the heart when they persist after birth. The infants affected are more or less cyanosed or blue in color, especially about the lips and finger-nails. There are periods when this is more marked than at other times. They are poorly. nourished and usually die in the first few months, if not from weakness of the heart, from some intercurrent condition. At times they live, the openings closing late and the normal circulation being established.

Abnormalities in the origin of the large vessels are sometimes seen.

Transposition of the heart is sometimes noted. In this condition the heart is found on the right side instead of the left.

Fetal Endocarditis. - This is an inflammation of the lining of the heart which occurs before birth. It is nearly always the right side of the heart that is affected. After birth inflammations of the endocardium attack the left side. The fetal inflammation causes valvular lesions at the tricuspid and the pulmonary orifices, the children bcing born with these defects. It is safe to say that valvular lesions of the right side of the heart are always congenital.

Phenomena of the Action of the Heart.-The apexbeat is a pulsation caused by the apex of the conicshaped muscle of the heart coming in contact with the chest wall. After seven years of age it is normally situated in the fifth intercostal space, one-half to one inch inside of the mammary line. Before this time it is found higher and further to the left. 
The apex-beat may be displaced by such conditions as a pericardial effusion, a hypertrophy or dilatation of the heart, or the pressure from either side from such conditions as a pleural effusion, tumors, etc. Changes are noted in the force and extent of the apex-beat under similar conditions.

The normal area of heart dulness obtained by percussion is from the junction of the third rib, with the sternum on the left side, to the apex-beat which can usually be seen in the fifth interspace, from one-half to one inch inside the mammary line, from the apex-beat to the xiphoid cartilage (the lower end of the sternum or breastbone), and thence up the right border of the sternum to the level of the third rib.

The two sounds heard over the heart upon auscultation are caused as follows: The first results from the contraction of the ventricle and the impact of the heart against the chest wall, and is synchronous with the apexbeat (called the systolic sound). The second sound is caused by the closure of the aortic and pulmonary valves (diastolic). The first sound is long and booming, and between it and the second sound there is a slight pause. The second sound is short and high pitched. After the second sound a long pause follows before the first sound is repeated; characterized by "lubb, tub." Any alteration in the character of either of these sounds is spoken of as a murmur. Accentuation of the sounds does not constitute a murmur. If the murmurs are due to a disease of the heart proper they are spoken of as endocardial murmurs. If due to anemia they are called hemic murmurs. In pericarditis they are called friction sounds, and in aneurism, bruit. 
A hemic murmur is a soft, blowing sound heard over the base of the heart. They do not indicate any damage to the valves; only an alteration of the blood, such as the diminution of hemoglobin found in anemia.

Flint's murmur is the name given to a sound heard in aortic regurgitation, due to the mitral leaflets vibrating between the column of blood passing through the mitral orifice and the column leaking back from the aorta.

The locations where the different valve sounds are best heard are:

Mitral, at the apex; tricuspid, at the end of the sternum (xiphoid cartilage); aortic, second costal cartilage, about an inch to the right of the sternum; pulmonary, second costal cartilage, about an inch to the left of the sternum.

The Pulse.-At birth the pulse is between I 30 to I 50 per minute, in the second year about roo, and gradually lessens as the child matures. An adult has a pulse of 70 or 80 per minute.

There are several changes which take place in the pulse which have received different terms.

Tachycardia, an increased frequency of the pulse.

Bradycardia, infrequency of the pulse.

Intermittent, dropping a beat.

Irregular pulse, alternately rapid and slow.

A dicrotic pulse is one in which the main beat is quickly followed by a secondary wave or a slight rebound of the vessel. It is seen in the cases where there is a low arterial tension, especially in febrile diseases. It may cause the mistake of counting double the number of beats actually present. 
A high-tension pulse is one in which the force of the beat is relatively increased. The tension of the pulse may be estimated roughly by noting the amount of pressure of the fingers that is required to arrest the beat. A low tension pulse is just the opposite.

Venous pulse, when present, is usually found over the jugular vein. It is rare.

Asymmetric Radial Pulse.-When the two radial beats are not synchronous it may be due to conditions affecting one side of the circulation, as aortic aneurisms, fractures, luxations, etc.

Water-hammer pulse is characterized by short, powerful beats which suddenly collapse. It is seen in aortic regurgitation (see page $\mathrm{I} 68$ ).

Other conditions which are present in diseases of the heart beside the alteration of the apex sounds and pulse above noted, are:

Palpitation, which is a rapid tumultuous action of the heart perceptible to the patient. This condition is termed a functional disorder.

Dropsy.-An unnatural collection of serous fluid in the tissues of the body.

General Cyanosis.-Blueness of the surface from insufficient oxidation of the blood.

Clubbing of the fingers in chronic cases.

\section{PERICARDITIS}

This is an inflammation of the pericardium or serous covering of the heart. It is rare in infancy, but as the child grows older it is not uncommon. It follows pleuropneumonia, rheumatism, the acute infectious diseases, pyemia, tuberculosis, and local causes. 
Pathology.-The same conditions exist in pericarditis as in inflammations of a serous membrane in any other part of the body. At first the surfaces are red and sticky; they rub together and cause a great deal of pain. In a day or two an effusion appears, which may be serous, serofibrinous, fibrinous, or purulent.

In the serofibrinous form there is little lymph and a great deal of serum, which, in favorable cases, is absorbed. In the fibrinous form, just as in pleurisy, the surfaces

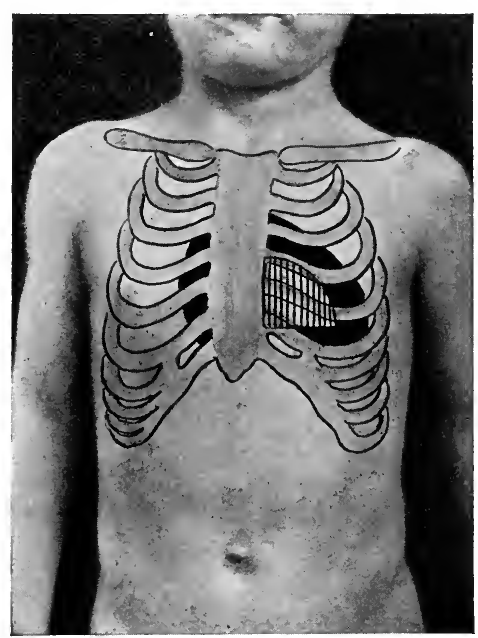

Fig. 44--Large pericardial effusion. Area of dulness in solid black. Normal area of absolute cardiac dulness lined (Kerr).

are covered with a butter-like exudate which may organize and form adhesions. In the purulent form death is usually the result.

Symptoms.-Moderate fever, precardial pain and tenderness, dyspnea, and palpitation. The pulse is at 
first rapid and forceful, but later weak and irregular. The signs of the effusion will appear on the second or third day, and there will be relief from pain in all but the fibrinous form, where it is apt to be intensified, owing to the adhesion of the two surfaces. The disease lasts from one to three weeks.

In the adhesive form of pericarditis, more or less permanent enlargement of the heart results.

Treatment.- The physician may tap the effusion, in which case the nurse should prepare for the operation in the same manner as described for aspirating the chest (see page 77 ), the precardia being the point of insertion instead of the axilla.

Other affections of the pericardium noted are:

Hydropericardium, dropsy withir the pericardial sac; hemopericardium, blood within the pericardial sac; pneumopericardium, air within the pericardial sac.

\section{ENDOCARDITIS}

Endocarditis is an inflammation of the lining membrane of the heart. The process is usually confined to the valves.

Varieties.-Vegetative, in which are found numerous bead-like vegetations that are especially marked along the free border of the valve. The valve itself is red and swollen. These vegetations are composed of connective tissue and fibrin, the latter derived from the blood. They may be whipped off by the blood current and may be carried as emboli to distant organs, such as the brain, kidney, or spleen. But more commonly if life is preserved they are partially absorbed and fibrous tissue forms, causing a hardening or sclerosis of the valve leaflets. 
This forms the second variety of endocarditis, the socalled sclerotic or chronic endocarditis. The latter may arise as a primary disease, and is then characterized by the thickening, curling, and puckering of the valves from an overgrowth of the fibrous tissue.

The third variety is the so-called malignant or ulcerative endocarditis.

Acute endocarditis usually results from acute articular rheumatism, one of the infectious fevers, chorea, or septicemia. Forty per cent. of the cases of articular rheumatism have associated endocarditis. Of the infectious fevers, pneumonia and scarlatina are most prone to heart complications. The young are more liable to be attacked than the old.

Chronic endocarditis may be congenital, the right side of the heart being then affected. It may follow an acute attack or it may result directly from syphilis, rheumatism, or Bright's disease. Severe muscular strain sometimes induces it in older children.

After birth endocarditis most commonly involves the valves of the left side of the heart.

Symptoms of Acute Endocarditis.-Subjective phenomena, such as pain, fever, cough, and dyspnea, are frequently absent, and auscultation may be the only means of diagnosing the disease, should a murmur be found. In many cases fever, an irregular and rapid pulse, palpitation, precordial distress, and dyspnea are associated symptoms.

To understand the conditions which cause valvular lesions of the heart the pathologic changes which take place in endocarditis should be kept in mind.

It is safe to say that in the majority of cases which survive the acute attack the sclerotic form of endocarditis 
exists. The sclerotic form is an overgrowth of fibrous tissue which causes the leaflets of the valves to become swollen and permanently thickened. This enlargement of the leaflets obstructs the valvular orifice. This is the case in the so-called stenosis or obstructive valvular conditions. This fibrous tissue sometimes contracts, in which condition the valve becomes wrinkled, puckered, and curled up. It is then easily discernible that a valve thus affected could not close the valvular orifice completely, and the blood leaks back into the chamber it has just left. This is the condition found in insufficiencies or regurgitations.

As there are four valves to the heart-aortic, pulmonary, mitral (left side), and tricuspid (right side)there may be an aortic stenosis or an aortic regurgitation; a pulmonic stenosis or a pulmonic regurgitation; a mitral stenosis or a mitral regurgitation; a tricuspid stenosis, or a tricuspid regurgitation.

Murmurs.-A murmur is any alteration in the normal sound of the heart. (Accentuation of sounds is not considered a murmur.) A murmur is produced by the blood flowing over a roughened or damaged valve. In an obstruction to an orifice the opening is too small, and in forcing the blood through the narrow passage it produces a blowing sound or murmur. In insufficiencies where the valve is not tightly closed there is a leakage back into the chamber of the heart from which the blood has been pumped after the valves are closed.

This passage of blood through the leak causes the same blowing sound or murmur.

The murmur occurs at the time the blood passes through the damaged valve. In obstruction it is at the time the blood is flowing from the chamber into the next 
portion of the circulation. If the obstruction is at the orifice between the auricle and the ventricle, it is at the moment the auricle is emptying itself into the ventricle. If the obstruction is at the entrance to the aorta or pulmonary artery, the murmur occurs when the ventricle is emptying into the vessels.

In regurgitations the orifice is not too narrow, but "rather larger than normal, the valves being wrinkled up; so there is no murmur when the blood is passing from one chamber into the next part of the circulation. When the valves close to prevent back-flow, they do not close the opening entirely; consequently there is leakage into the chamber from which the blood has just passed. This leak is small and it causes a murmur at the time when the valves should be closed.

The time when the various phenomena of the heart's action occurs is divided into systole and diastole.

Systole is the time when the ventricles empty, the blood flowing into the aorta and the pulmonary artery. The mitral and tricuspid valves are closed to prevent the blood from flowing into the auricles. The auricles fill while this is taking place.

The heart-beat occurs at this time, demonstrated by the apex-beat, the pulse wave, and the first sound of the heart.

Diastole is the time when the aortic and pulmonary valves close to prevent the blood from flowing back into the ventricle, causing the second sound of the heart; the mitral and tricuspid valves open, and the blood flows from the auricles into the ventricles. The time of the different murmurs is as follows:

Mitral stenosis or obstruction occurs when the blood 
is flowing from the left auricle into the left ventricle. The time is diastolic. As the greatest contraction of the auricle occurs late in the diastole the murmur is heard best at that time. It is called a late diastolic murmur, or, more often, presystolic. It is heard just before the first sound of the heart at the apex.

Mitral regurgitation or insufficiency occurs when the blood should be flowing from the left ventricle into the aorta. A portion leaks back through the damaged mitral valve. The time is systolic. It is heard instead of the first sound at the apex.

Aortic stenosis or obstruction occurs when the blood flows from the left ventricle into the aorta. The time is systolic. It is heard instead of the first sound at the aortic area, the second costal cartilage to the right of the sternum.

Aortic regurgitation or insufficiency occurs when the aortic valves should be completely closed. This leak allows the blood to flow back into the left ventricle. The time is diastolic. It is heard instead of the second sound of the heart at the aortic area.

Tricuspid stenosis or obstruction occurs when the blood is flowing from the right auricle into the right ventricle. The time is late diastolic or presystolic. It is heard just before the first sound at the end of the sternum (xiphoid cartilage).

Tricuspid regurgitation or insufficiency occurs when the blood should be flowing from the right ventricle into the pulmonary artery, and the tricuspid valve is closed. A portion leaks through the damaged valve into the right auricle. The time is systolic. It is heard instead of the first sound at the end of the sternum. 
Pulmonary stenosis or obstruction occurs when the blood is flowing from the right ventricle into the pulmonary artery. The time is systolic. It is heard instead of the first sound at the pulmonary area, at the second costal cartilage, one inch to the left of the sternum.

Pulmonary regurgitation or insufficiency occurs when the pulmonary valve is closed to prevent the back-flow of blood into the right ventricle. The damaged valve permits a portion of the blood to leak back into the right ventricle. The time is diastolic. It is heard instead of the second sound at the pulmonary area.

Double murmurs are heard when one or more lesions exist, the valves affected being determined by the time of murmur, and the location where they are best heard. Under such conditions both a systolic and a diastolic murmur can exist.

Reduplication of the second sound is due to the fact that the aortic and pulmonary valves do not close simultaneously, the closure of both valves being heard.

Mitral regurgitation is the most common valvular lesion.

Table of Heart Murmurs

\begin{tabular}{|c|c|c|c|c|}
\hline Lesion & Systolic & Diastolic & Presystolic & Heard best \\
\hline $\begin{array}{l}\text { Mitral stenosis } \\
\text { Mitral regurgitation } \\
\text { Aortic stenosis } \\
\text { Aortic regurgitation } \\
\text { Tricuspid stenosis } \\
\text { Tricuspid regurgitation } \\
\text { Pulmonary stenosis } \\
\text { Pulmonary regurgitation }\end{array}$ & $\begin{array}{l}+ \\
+ \\
+ \\
+\end{array}$ & + & + & $\begin{array}{l}\text { Apex } \\
\text { Apex } \\
\text { Aortic cart. } \\
\text { Aortic cart. } \\
\text { Xiphoid } \\
\text { Xiphoid } \\
\text { Pul. cart. } \\
\text { Pul. cart. }\end{array}$ \\
\hline
\end{tabular}

The period of compensation means an increase in the size and strength of certain cardiac chambers, sufficient 
to enable the arterial system to receive its normal amount of blood, notwithstanding obstruction or regurgitation at one or more valves.

The duration of this period is indefinite, and depends largely upon the amount of damage sustained by the heart and the hygienic conditions to which the patient is subjected. It is Nature's way of overcoming the damage done. In the lung this is accomplished by compensatory emphysema; in the heart the same amount of work has to be continuously accomplished. If a portion of the heart is disabled, the rest of the heart has to do the extra work, and therefore becomes enlarged and hypertrophied. During perfect compensation the murmur is usually the only sign of the endocarditis. People frequently go through life with a murmur and live as long as those who are not so unfortunate. As long as there is perfect compensation there is no danger to the life of the patient.

Periods of lost compensation usually result from increased damage to the valves, conditions leading to arterial and cardiac degeneration, some intercurrent disease throwing additional strain upon the heart, and undue physical exertion.

During this period the subjective symptoms reappear. This loss of compensation gives rise to dilatation of the heart and cardiac insufficiency. No matter what the original valvular lesion may have been, the organ becomes unable to fill the arteries and the blood is dammed back in the lungs and venous congestion of the organs follows. Very frequently the heart will readjust itself and compensation will return.

Acute ulcerative endocarditis is a rapidly destructive form of endocarditis and is characterized by necrosis 
or ulceration of the valves. It usually follows septicemia or one of the infectious fevers, such as pneumonia, erysipelas, or scarlet fever. It may arise as a primary condition. The valves are the seat of ulcers, deep abscesses, and soft yellowish vegetations which have undergone partial necrosis. Microscopic examination reveals myriads of micro-organisms.

Symptoms.-They are divided into three classes: General, cardiac, and embolic.

General Symptoms.-There is a high and irregular fever, repeated chills, profuse sweats, great prostration, often delirium and stupor, hurried breathing, a rapid irregular pulse, and a brown, fissured tongue. Jaundice and diarrhea are frequently present.

Cardiac Symptoms.-Precordial pain, palpitation, and often murmurs at one or more valves. These may be absent.

An embolism is a small portion of the vegetation formed on the valve leaflets which becomes loosened and is swept into the circulation by the force of the blood current. It is carried through the arterial system until it lodges in a vessel of too small caliber to permit its passage. In this position it entirely plugs up the artery and prevents the passage of blood. This causes the part supplied by the arteries to be cut off from its blood-supply.

Peripheral emboli yield a petechial rash; renal embolism may yield a bloody urine, splenic embolism may yield a painful spleen; cerebral embolism may yield paralysis. The disease lasts from a few days to five or six weeks. Death almost invariably results. 


\section{MYOCARDITIS}

Acute myocarditis is an acute inflammation of the heart muscle. It is rare in childhood. It is almost always secondary to endocarditis or pericarditis. As a primary affection of the heart it may be due to rheumatism or to one of the infectious fevers. The heart muscle becomes degenerated, flabby, and friable.

The symptoms are those characteristic of any heart condition, and include pain, dyspnea, a very rapid and irregular pulse, and since the heart muscle itself is weakened, the heart-beats are weak and, in addition, the pulse very small.

Fibroid heart is the term given to a chronic myocarditis; it is characterized by an overgrowth of connective tissue, just as in any chronic inflammation elsewhere.

\section{HYPERTROPHY OF THE HEART}

Hypertrophy of the heart is an enlargement of the heart due to an overgrowth of its muscle. It is caused by increased work, and this may be due to too much blood to be removed from the heart, as in regurgitant valvular lesions; obstruction to the outflow of the blood at the valves, as in the stenoses or obstruction in the pulmonary or systemic circulation, as in emphysema or Bright's disease; resistance to ventricular contraction, as in adhesions; undue physical exertions, long continued.

Varieties.-Simple hypertrophy comprises a thickened muscle with a normal cavity. Eccentric hypertrophy (hypertrophy with dilatation) consists of a thickened muscle and dilated cavities.

Concentric Hypertrophy.-There is a thickened muscle and the cavities are diminished in size (always congenital). 
Dilatation of the heart is an enlargement of the heart due to the stretching of its walls.

Varieties.-Dilatation with thickening of the walls, which is the same as eccentric hypertrophy; dilatation with thinning of the walls.

Dilatation results from excessive pressure within the chambers of the heart, as from sudden extreme exertion; in valvular disease; and in impaired nutrition of the heart muscle, as in low fevers, valvular disease and sclerosis, or hardening of the arteries of the heart. One or both ventricles may be dilated, but usually the right.

Symptoms.-As long as the associated hypertrophy keeps pace with the dilatation there are no symptoms, but when the dilatation preponderates, the following symptoms of venous stasis appear: Dyspnea and cough, the blood being dammed back in the venous system causes engorgement of the lungs; scanty urine from congestion of the kidneys; dyspepsia from congestion of the stomach. There is general dropsy, and feeble, irregular pulse.

Fatty degeneration of the heart is a changing of the heart muscle into fat. When fat is merely deposited upon the heart muscle it is termed fatty infiltration. Fatty degeneration is due to sclerosis of the coronary arteries, valvular diseases, infectious fevers, and to certain poisons, as arsenic, phosphorus, and antimony.

Symptoms are those of heart failure: Dyspnea, asthma, cough, weak, irregular pulse, poor digestion, dropsy, syncope, and Cheyne-Stokes respiration.

\section{NURSING OF CARDIAC DISEASES}

Absolute rest is of the greatest importance; light and nutritious diet, improvement of the general condition by 
careful hygienic surroundings. Ice to the precordia will frequently relieve pain.

Bathing should be accomplished only by sponge baths.

In a severe case of heart disease the child should lie on its back without a pillow. When dyspnea is marked it is necessary to prop up the child in bed so that it can breathe with less discomfort (orthopnea).

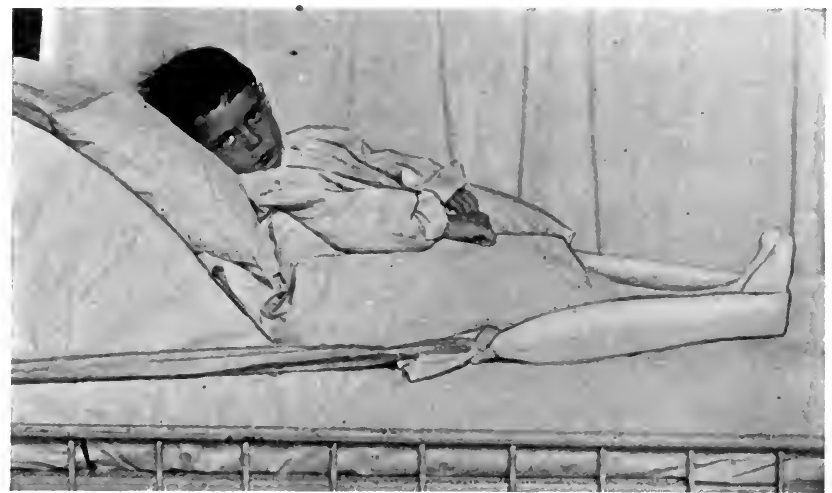

FIG. 45.-Position in orthopnea. The baby is supported by a frame, a pillow is placed on both sides to support elbows, and a folded sheet is passed around feet to prevent child from slipping down. The sheet is held in position by bandages attached to head of bed.

The temperature, pulse, and respirations should be taken at least twice a day. It may be necessary to take the pulse more often, and in severe cases the pulse should be under constant observation.

Symptoms of heart failure demand instant attention. Whenever the pulse becomes rapid, intermittent, and weak, or the child has sudden attacks of dyspnea, coldness of the extremities, or attacks of syncope, the physician should be immediately informed. While awaiting his arrival the 
child should be placed flat upon its back and not moved for anything. Mustard paste, made of equal parts of mustard and flour, may be applied to front of chest until there is a distinct redness, I5 minims of aromatic spirits of ammonia may be given in water by the mouth, if the child can swallow; hot-water bags may be placed about the extremities, and inhalations of ammonia given. (Be careful not to have concentrated ammonia nor to hold it continuously under the nose; pass it slowly backward and forward.) The physician will probably order a hypodermic injection of one of the following drugs: nitroglycerin, strychnin, digitalis, or whisky, all of which should be in readiness. Hypodermoclysis may be ordered and at times he may bleed the child.

\section{DISEASES OF THE BLOOD-VESSELS AND THE BLOOD}

Aneurism is a circumscribed dilatation of an artery. It is uncommon in childhood. The cases reported have been due to hereditary syphilis.

The arch of the aorta is the most frequent seat; there may be abdominal aneurisms, and also aneurisms of any artery of the body.

Symptoms.-Thoracic aneurisms: Dyspnea, metallic cough, pain, difficulty in swallowing, dilatation of superficial veins, unilateral sweating, paralysis of the sympathetic nerves, and the presence of a tumor.

Arteriosclerosis is a thickening of the arteries due to an overgrowth of connective tissue, associated with more or less fatty degeneration and hardening. Not common in childhood. 


\section{BLOOD}

In health the blood amounts to about one-thirteenth of the body-weight. It is composed of serum (a watery fluid), red blood-corpuscles, and white blood-corpuscles.

Hemoglobin is the coloring matter of the red bloodcorpuscles; it is principally composed of iron. Its function is to carry oxygen to the tissues of the body.

Estimation of the Red and White Corpuscles.-The number is obtained by accurate mathematical calculation. The instrument used is called a hemocytometer.

The blood withdrawn into the capillary tube is accurately diluted and a drop of this is placed upon a measured glass slide of known dimensions. Under the microscope the number of red and white corpuscles is counted in the measured area. The dilutions being known, the number of corpuscles in a cubic millimeter can be deduced.

The hemoglobin is estimated by means of an instrument called a hemoglobinometer.

There is a prism of colored glass so arranged that onehalf of a small circular receptacle stands above it, containing clear water. The other half contains diluted blood. By means of a reflected light and the movable prism, which is graded from a light to a deep reddish hue, the two sides are so adjusted that they will be of the same shade. The percentage is read from a small scale attached to the sliding prism. Normal blood will read soo per cent. on this instrument.

'The specific gravity of the blood is obtained by placing a drop of blood in a fluid mixture of known specific gravity. When the drop remains stationary the specific gravity is the same as the mixture. 
Normal blood contains 5,000,000 red blood corpuscles to a cubic millimeter.

At birth there are about 18,000 , and later in childhood from 6,000 to I2,000 white biood-corpuscles to a cubic millimeter. The specific gravity is about $\mathrm{I} .055$ :

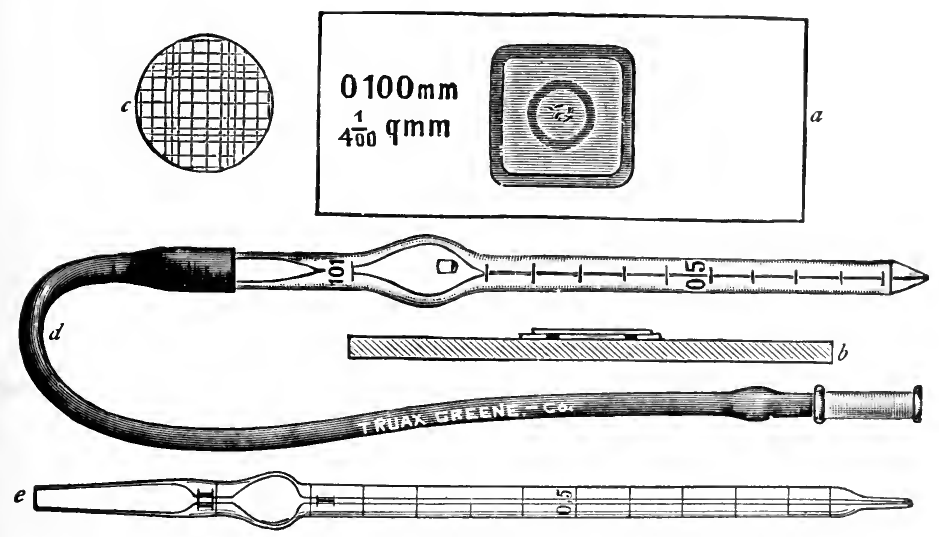

Frg. 46.-Thoma-Zeiss hemocytometer : $a$, Slide used in counting; $b$, sectional view; $c$, portion of ruled bottom of well; $d$, red pipet; $e$, white pipet.

Anemia is a condition of the blood in which it is deficient in quantity or in one or more of its constituents.

It is evidenced by pallor of the skin and mucous membrane, and by progressive weakness. Iron is used as the remedy, its action tending to increase the percentage of iron in the depleted hemoglobin.

Pernicious anemia is a grave form of anemia characterized by a great deficiency in the number of red bloodcorpuscles, and not associated with any definite causal lesion.

Plethora, is an increase in the whole quantity of blood. 
Leukocytosis is an increase in the number of white blood-corpuscles.

Leukemia is a disease characterized by a great increase in the number of white blood-corpuscles, with lesions of the spleen, lymphatic glands, and bone-marrow.

Chlorosis is a disease in which there is a great reduction in the hemoglobin (coloring matter), without any decrease in the red blood-corpuscles. It derives its name from the green tint of the skin.

Von Jaksch's disease is characterized by a decrease in the red blood-corpuscles and hemoglobin, and by marked leukocytosis with enlargement of the spleen and at times the liver. 


\section{CHAPTER IX}

\section{NERVOUS DISEASES}

\section{ANATOMY OF THE NERVOUS SYSTEM}

THE central nervous system is composed of the brain, the spinal cord, and their coverings.

The brain is contained within the cavity of the skull. It is the center of thought, of the perception of the five senses, and of the voluntary motor activities of the body.

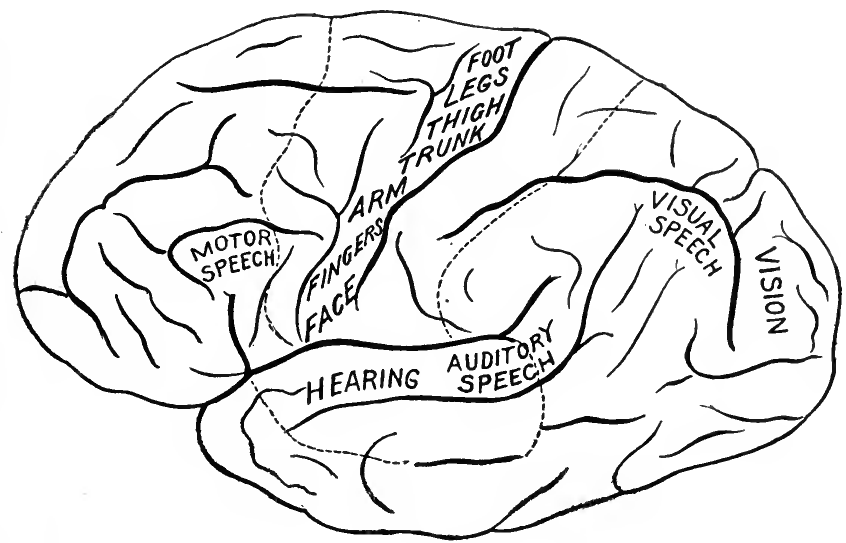

Fig. 47--Functional areas of the cerebral cortex, left hemisphere (A. A. Stevens).

The brain or cerebrum is divided into the right and left hemispheres by the median fissure. The two hemispheres are united by fibers running through the corpus callosum. 
The lower portion is divided into the cerebellum, the

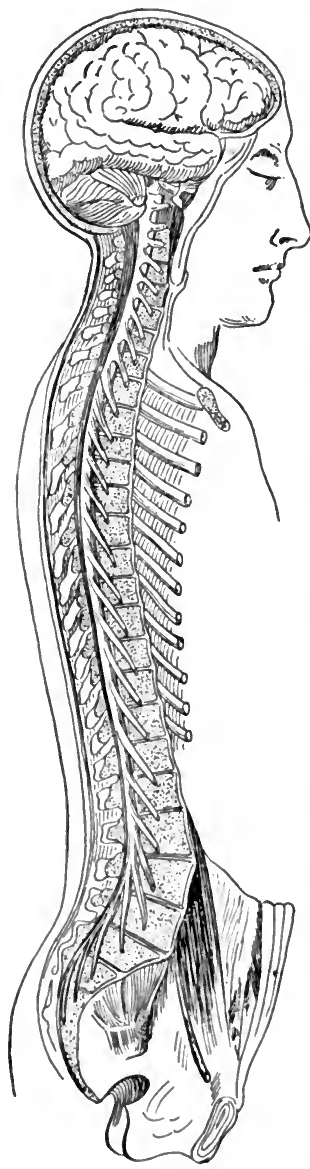

Fig, 48-Cieneral view of the cerebrospinal nervous system (after Bourgery; Schwalbe). crura, the pons, and the medulla.

The brain is composed of gray and white matter, the gray matter being external and about a half inch in thickness; it is called the cortex. The rest of the brain is made up principally of white matter and consists of nerve fibers running in various directions.

The cortex presents upon its surface dcep grooves, which are called fissures or sulci. These fissures are numerous and divide the brain into convolutions. This arrangement gives the greatest area of cortex in the smallest amount of space. The more developed the convolutions, the greater the intellect of the individual. The fissures also divide the brain into different lobes, such as the frontal, temporal, and parietal lobes.

The fissure of Sylvius is a large sulcus on the external lateral surface of the brain, and it receives a portion of the wing of the sphenoid bone.

The fissure of Rolando runs from the center of the vault of the skull, downward and forward toward the ear. Around 
this fissure are located the motor centers of the body; that is, the nerve cells which form the nervous impulses, making voluntary motion possible.

The arrangement of the motor centers is "upside down"; that is, the center for the legs is uppermost; then, in order, the center for the trunk, the arms, the face, and head, the last-named being the lowest in location. (See Fig. 47.)

The nerve fibers run from the centers around the fissure of Rolando, through the internal capsule (a pathway in the substance of the brain), to the crura. A hemorrhage within the internal capsule is the cause of hemiplegia. The blood-clot, by exerting pressure, prevents any passage of nervous impulses, producing paralysis of the muscles supplied.

The crura are two extensions of the brain, one from each hemisphere, which carry motor and sensory fibers. They unite and carry the fibers from both hemispheres as far as the pons.

The pons is a bridge of nervous tissues. It not only contains the motor and sensory fibers from the cortexcarrying them from the crura to the medulla, the next portion of the nervous system below-but it also contains fibers running between the two hemispheres of the cerebellum.

The medulla or bulb is the lowest portion of the brain, and at the foramen magnum it becomes the spinal cord. The motor and sensory fibers decussate or cross from one side to the other in the medulla. On account of this crossing of fibers an injury to the left side of the brain produces a paralysis of the right side of the body (hemiplegia). The medulla contains the center of the involuntary movements, as respirations, etc. 
The motor fibers run through the anterior and lateral columns of the spinal cord and the sensory fibers through the posterior columns. At the level of the muscle to be supplied the motor fiber leaves the cord by one of the spinal nerves and runs through the branch of this nerve to the muscle. 'The sensory fibers run from the skin surface and return to the cord through the spinal nerve and enter the posterior columns of the cord.

In the cerebellum is located the center of co-ordination. These centers keep all portions of the body working together in unison.

The basal ganglia are isolated areas of gray matter within the white matter of the brain, and are associated with the special senses.

The ventricles of the brain are cavities within its substance. There are four in number, all connected and containing cerebrospinal fluid.

The spinal cord is contained within the spinal column and is a continuation of the medulla. The pathways of the motor and sensory fibers are external, the gray matter being located in the center in the form of an $\mathrm{H}$.

The cord is divided into columns. The posterior column receives the sensory filaments through which they run on their way to the brain.

The lateral columns and the anterior columns are composed of motor filaments running from the brain to the muscle.

The central area of gray matter contains trophic cells in addition to motor fibers.

Trophic cells supply the tissues with proper nervous tone; when they are injured bed-sores develop and atrophy of the muscles occurs. 
The spinal cord contains most of the centers of reflex action.

Since the posterior columns carry only sensory fibers, injury or disease of this part of the cord will cause a loss of sensation below the lesion (locomotor ataxia).

Since the anterior and lateral columns carry only motor fibers, injury or disease of this part of the cord will cause paralysis below the lesion (myelitis). .

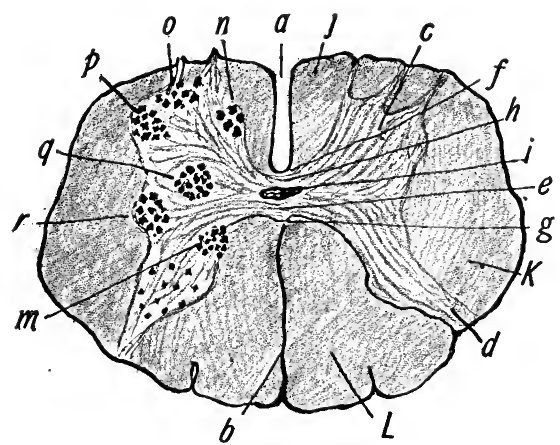

Fig. 49.-Lumbar section of spinal cord showing main tracts of white substance and location of principal groups of nerve-cells in gray matter: $a$, Anterior median fissure; $b$, posterior median fissure; $c$, anterior horn of gray matter; $d$, posterior horn of gray matter; $e$, central canal; $f$, anterior white commissure; $g$, posterior white commissure; $h, i$, anterior and posterior gray commissures; $j$, anterior median column; $K$, lateral column; $L$, posterior column; $m$, column of Clarke; $n$, inner group of nerve-cells; $o$, anterior group; $p$, anterolateral group; $q$, posterolateral group; $r$, lateral horn (Leroy).

The Meninges.-The brain and spinal cord are covered by three membranes: the dura, the arachnoid, and the pia.

The dura is a thick, fibrous structure lining the cavities of the skull and spinal canal, dipping into the median fissure and separating the cerebellum from the cerebrum. It also forms the venous sinuses of the brain and surrounds 
the cranial nerves. The function of the dura is to act as a protection to the structure enclosed.

The arachnoid is a serous membrane and is very similar to the pleura and the pericardium. It surrounds the brain and cord, and is reflected so that there are two surfaces between which there is a closed sack, containing the cerebrospinal fluid. 'The sack is continuous with the ventricles, of the brain. This arrangement not only lubricates the slight movements of the cord and the brain, but permits the central nervous system to rest on a cushion of water, which annuls many shocks.

The pia is a thin meshwork of blood-vessels. It closely surrounds the brain and the spinal cord, dipping into the fissures and ventricles, and is the main blood-supply of the cortex and cord.

The cranial nerves are twelve in number: (I) olfactory, (2) optic, (3) motor oculi, (4) pathetic, (5) trifacial, (6) abducens, (7) facial, (8) auditory, (9) glossopharyngeal, (IO) pneumogastric, (II) spinal accessory, (I2) hypoglossal.

They supply the organs of the special senses, the structures of the face, the head and the neck, and, through the pneumogastric, the lungs, the heart, and the stomach.

The spinal nerves consist of thirty-one pairs. They leave the spinal cord at various levels and carry motor fibers to, and sensory fibers from, the trunk and the upper and lower extremities. The nerves supplying the various structures of the above parts of the body are branches of the spinal nerves.

The sympathetic nerves control involuntary actions and keep the different parts of the body working smoothly together (co-ordination). 


\section{TERMINOLOGY}

The disturbances of motion are paralysis, convulsions, choreiform movements, and tremors.

Paralysis may involve one member only, and it is then termed monoplegia; a lateral half of the body, when it is termed hemiplegia; or it may involve the body from the waist down, when it is called paraplegia.

A convulsion is a condition in which there are excessive muscular contractions, continued or intermittent, dependent upon the involuntary discharge of the motor impulses from the nerve centers.

Intermittent contractions are termed clonic; continued contractions, tonic. Convulsions may be general or local. The term spasm is sometimes applied to the latter.

Varieties of convulsions: Epileptiform, tetanic, and hysteroidal.

Epileptiform convulsions are characterized by unconciousness, and for the most part the movements are clonic. They are preceded by an aura, and the patients bite their tongues.

Tetanic Convulsions. - In this form the discharges emanate from the spinal cord and the convulsive movements are continuous and not associated with unconciousness.

Hysteroidal convulsions are manifestations of hysteria, and in them consciousness is only partially or apparently lost. They are not preceded by an aura, but sometimes by a sensation as of a ball in the throat. The eyes are partially closed, the face expresses some emotion, the tongue is not bitten, the movements are tonic, or, if clonic, 
appear wilful. The paroxysm is of long duration and the patient frequently weeps or laughs.

There are various local spasms, such as hiccough, croup, and laryngismus stridulus, etc.

Choreiform movements are coarse, jerky, irregular, involuntary movements, which more or less simulate purposeful movements.

Athetosis is the term applied to certain movements of the hands and feet in which there is a slow, twisting, interwinding, separation and extension of the fingers and toes. It is frequently observed in the cerebral palsies of children.

Tremors are fine, vibratory movements due to the alternate contraction and relaxation of antagonistic groups of muscles.

The knee-jerk is obtained by tapping the quadriceps tendon between its insertion and the patella while the legs are crossed. The value of the knee-jerk depends upon the mechanism of its production. This is called the reflex arc. It consists of the sensory nerve running from the patella tendon to the spinal cord, where it enters the reflex center. From this center the motor nerve runs to the muscle, causing it to contract. If the arc is broken by disease or injury to any one of its parts the knee-jerk is lost. When there is any irritating lesion of the cord the reflexes are increased. When there is any destructive lesion of the cord the arc is broken and the knee-jerks are absent. They are, therefore, a very important diagnostic symptom.

Ankle clonus consists in a vibratory movement obtained by supporting the tendo Achillis with one hand while the foot is strongly flexed. 
Babinski's reflex is obtained by tickling the sole of the foot; if there is an injury or disease of the central nervous system the great toe will move upward instead of downward, as it does normally.

Sensation.-Anesthesia means loss of sensation.

Hyperesthesia means exaggerated sensation.

Paresthesia is used to indicate certain disagreeable sensations, such as numbness, tingling, itching, creeping, and feeling of "pins and needles."

Arthropathies are degenerative changes of the joints.

Coma is a condition of unconsciousness from which the patient cannot be aroused.

Temporary unconsciousness due to anemia of the brain is termed syncope.

Catalepsy is characterized by a peculiar stiffness of the muscles, and when this is overcome by force, the limbs can be placed in unnatural positions, which they retain for a long time. There may or may not be a loss of consciousness and sensation.

\section{PECULIARITIES OF DISEASE OF THE NERVOUS SYSTEM IN CHILDREN}

-Owing to the immature development of the central nervous system, and to the great irritability of the peripheral or terminal sensory nerves, much more serious nervous symptoms are shown by children from trivial causes than are seen in adult life. Hence, conditions such as convulsions, tetany, St. Vitus' dance, and enuresis are common.

The greatest factors in the cause of such conditions are stimulants, such as tea and coffee, and the fact that children live among exciting surroundings. Plenty of sleep 
and quiet are essential to the proper development of the nervous system. The effects of such conditions as infantile paralysis and birth palsies last through life.

Hemorrhage of the brain is usually cortical, on the outside of the hemisphere, and not within its substance.

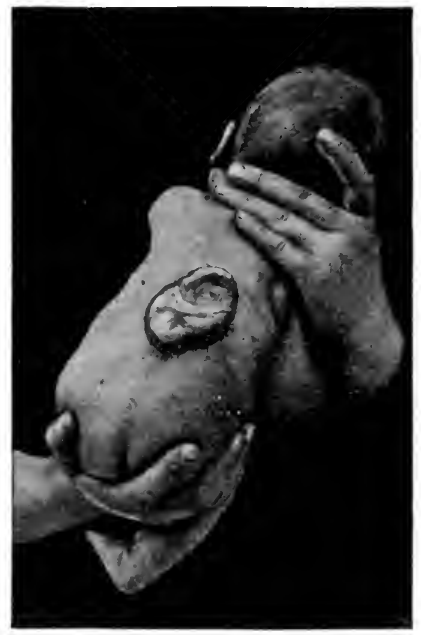

F1G. 50.-Spina bifida (Eisendrath).

Malformations.-Meningocele is a protrusion of the covering of the brain (meninges) through some abnormal opening in the skull or spinal canal. It contains cerebrospinal fluid. When they are spinal in origin they are spoken of as spina bifida.

Encephalocele is a protrusion of a portion of the meningus containing brain substance.

Hydro-encephalocele contains both brain substance and fluid.

Microcephalus is a name given to a small head due to under-development. 
Hydrocephalus is an enlargement of the skull due to a large amount of cerebrospinal fluid within the ventricles.

Other deformities are noted, such as absence of a whole or a part of the brain.

\section{Diseases of the Meninges MENINGITIS}

This term is applied to any inflammation of the membranes covering the brain and spinal cord. It may be acute or chronic, and occurs (I) as a complication of the infectious diseases, (2) following some local cause, and (3) epidemically.

The epidemic variety is also spoken of as epidemic cerebrospinal meningitis or spotted fever, and is described on page 245 .

When the membranes covering the brain are involved it is called cerebral meningitis; when the spinal cord is the seat of the disease it is spinal meningitis; more often both the brain and spinal meninges are involved, and it is then spoken of as cerebrospinal meningitis.

Pathology. - The membranes are serous in character, they surround the cranial nerves as they leave the brain, and the spinal nerves as they emerge from the cord.

When a serous membrane is diseased it first becomes very red and inflamed, and later there is an exudate.

The first stage produces intense irritation of all the surrounding tissues, hence in cerebral meningitis there is severe headache and involvement of the nerves of the special senses. This causes intolerance of sound and light. Later, when the exudate develops, it produces pressure, and instead of irritation there is paralysis of those parts supplied by the nerves subject to the pressure of the exu- 
date. Deafness and blindness are then found instead of the symptoms of irritation.

In spinal meningitis, at first, there is present a spasmodic condition of the muscles due to the irritation of the spinal nerves, this is followed, after the exudate develops, by paralysis due to the pressure. When both the cerebral and spinal meninges are involved we have a combination of the above symptoms. Acute meningitis is most often caused by the diplococcus of pneumonia, by infection from a suppurating wound, by the bacilli of the infectious fevers, by traumatism, and especially by the tubercle bacillus.

The prognosis of all forms of meningitis is very grave. The non-tubercular varieties occasionally recover. Tubercular meningitis is invariably fatal. In the epidemic variety, Flexner's serum has given wonderful results.

The picture of a case of meningitis is complete in the tubercular variety, and only that form will be given here.

Tubercular meningitis (cerebral) is an acute inflammation of the cerebral meninges excited by the tubercle bacillus.

In children the disease may be primary or secondary to a focus of tuberculosis in some other part of the body. The majority of cases are seen between the second and fifth year.

The basilar meninges covering the lower part of the brain are especially involved. The pons, crura, and medulla are covered with lymph which mats together in a common mass the adjacent nerves and blood-vessels. The fluid within the ventricles of the brain is increased.

Symptoms.-The disease usually begins insidiously with dulness and irritability on the part of the child. 
Sleep is disturbed. The child twitches, grinds its teeth, and starts up with a cry of alarm. When the disease is

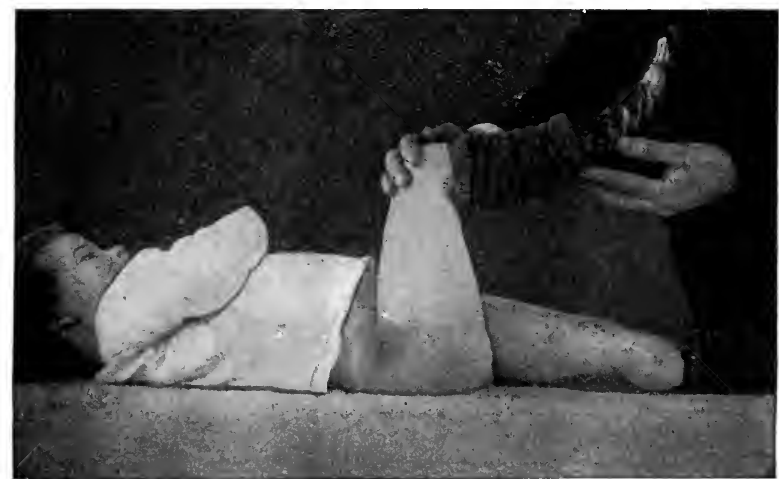

F16. 51.-Kernig's sign. The thigh is held at right angles to the body. When an attempt is made to extend the leg, bringing it into a line with the thigh, there is either marked resistance or an inability to extend the leg, if meningitis is present (Kerr).

fully developed headache is intense and causes a shrill scream, known as the hydrocephalic cry. The special senses are extremely acute, bright lights and noises cannot be tolerated; the child becomes irritable when touched. The temperature ranges between $102^{\circ} \mathrm{F}$. and $103^{\circ} \mathrm{F}$. The pulse is rapid at first, but later is slow and irregular. The walls of the abdomen are flat. The child lies on its side with the limbs drawn up, the head is bent far back, the fingers are clenched over the thumbs which are turned into the palm of the hand. This is called opisthotonos. Convulsions are common and may be local or general. Toward the close of the stage the child becomes delirious. When the exudate is of sufficient amount to exert pressure, paralysis develops, especially in the muscles of the face. Coma follows the delirium, the eyes are rolled up, and 
blindness and deafness result. If the finger is drawn across the body a bright red line develops and remains

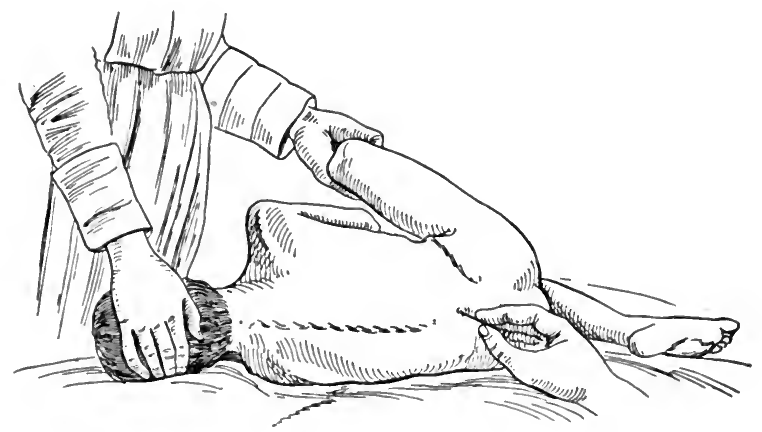

Fig. 52.-Method of introducing needle in lumbar puncture: Child in lying posture (Boston).

for some moments; this is called a tache. In the last stage the pulse becomes weak, rapid, and irregular; respirations assume the Cheyne-Stokes characteristics, and the temperature falls. The duration is from one to three weeks.

Kernig's Sign.-The inability to straighten out the leg when the thigh is bent upon the abdomen. It is present in cases of cerebral meningitis.

Lumbar Puncture.-During the course of the disease the physician may find it necessary to relieve the tension in the spinal canal, or he may desire to obtain fluid for diagnostic purposes. He then will tap the spinal canal by the lumbar puncture method. In preparing for this procedure the skin over the lumbar portion of the spine must be scrupulously sterilized and every aseptic precaution must be absolute. The child is usually held in the position as shown in Fig. 52. 'The method consists in inserting a long hypodermic needle between the vertebræ and through 
the membrane; as soon as it enters the spinal canal the cerebrospinal fluid runs out of the needle. Several sterile test-tubes should be in readiness to catch the fluid. When they are filled, plug them with aseptic cotton. The wound in the skin is usually closed with adhesive plaster or a collodion dressing.

\section{Diseases of THE BRAIN}

In diseases of the brain the centers for the various functions, such as motion, sensation, speech, hearing, sceing, smelling, and hearing, are interfered with. The pathways leading from the centers may be involved as they traverse the brain on their way to the spinal cord.

Diseases of the brain are usually diagnosed by what are termed pressure symptoms, produced by clots, tumors, abscesses, cysts, etc.

The minute anatomy of the brain is almost as well known as that of the spinal cord. The brain centers are definitely located and the direction of the pathways of the fibers from these centers is known. Therefore, it is possible to locate accurately a lesion of the brain, either a tumor, cyst, abscess, or morbid growth, from the pressure-symptoms which they produce. Areas of sclerosis and hemorrhage, destroying or impairing the centers and nerves or the tracts from the centers are determined in the same manner.

A lesion in a definite part of the brain will involve certain centers and nerves which will produce paralysis of the parts supplied by those nerves. Thus, if there is a hemorrhage or a tumor pressing on what is known as Broca's area in the brain, which is the speech center, there will be impairment or loss of the function of speech. If this 
symptom is present with other symptoms of cerebral involvement, such as persistant headache or unconsciousness, choked disk (a condition of the eye), and paralysis of other parts of the body, there is a tumor involving Broca's area.

A more accurate diagnosis than this can be made. The position of the motor areas around the fissures of Rolando are "upside down," the leg area above, the arm in the center, and the face below. The first symptoms of irritation to nervous structures are convulsions. Convulsions due to brain irritation of the motor areas are characteristic. They begin in the part that corresponds to that portion of the brain which is irritated. If the irritation is in the hand area of the motor region the convulsion will start in the hand, gradually extend up the arm, and then become general. This form of convulsion is termed Jacksonian epilepsy. A finer distinction than locating the lesion in the hand area can be made. If the positions in the different areas in the motor region are known, by watching this convulsion which starts in the hand, and by noting what parts are successively involved, the extent and direction of the convulsion can be determined. If, after the convulsive movements reach the shoulder, they involve the corner of the mouth it is plain that the lesion in the brain extends downward. If, on the other hand, the leg is the next part to become involved the lesion extends upward. This will impress the nurse with the necessity of observing a convulsion carefully so that the diagnosis can be accurately made, for brain surgery demands accuracy, and as so many lesions of the brain can be treated only by surgical means, its importance can be appreciated. 
A nurse will be able to observe convulsions more closely than anybody else; therefore, she should note the kind of convulsion, whether tonic or clonic, where it begins, what parts are successively involved, in which direction the eyes and head turn, for in destructive lesions the head and eyes are usually turned toward the side of the lesion of the brain, and in irritating lesions to the opposite side. Also note what parts of the face are involved, as the nerves supplying the face emerge from the skull at different levels. A lesion at one level might involve a nerve after it had crossed, giving a paralysis on the same side as the lesion instead of the opposite, as is the rule, producing the socalled crossed paralysis; while at another level it may be affected before it has decussated, giving a paralysis on the opposite side of the face. This knowledge gives aid in the determination of the level of the lesion. Also note whether the eyelids are drooped and whether the patient is conscious or unconscious.

\section{CONVULSIONS}

Convulsions occur frequently in childhood. They are due to direct irritation of the cortex or to reflex irritation. The poisons generated by the acute infectious diseases may so irritate the cortex that convulsions occur during the course of these diseases. Convulsions often usher in an attack of illness in children.

Reflexly, indigestion, teething, and other trivial causes at times produce general convulsions. This is due to the instability of the nervous system at this early stage.

Treatment.-A mustard tub, temperature $110^{\circ} \mathrm{F}$., for five minutes is the best method to employ (see page 407). The tongue should be protected by inserting something between the teeth and an enema given immediately. 
196 DISEASES OF CHILDREN FOR NURSES

\section{CEREBRAL PARALYSIS}

Birth palsy in children is not uncommon. It is caused by a hemorrhage upon the cortex of the brain, rarely within the brain substance. The hemorrhage usually occurs during the birth of the child.

The resulting paralysis may be a hemiplegia, half of the body being involved. Contractures occur and the children are usually mentally deficient and crippled.

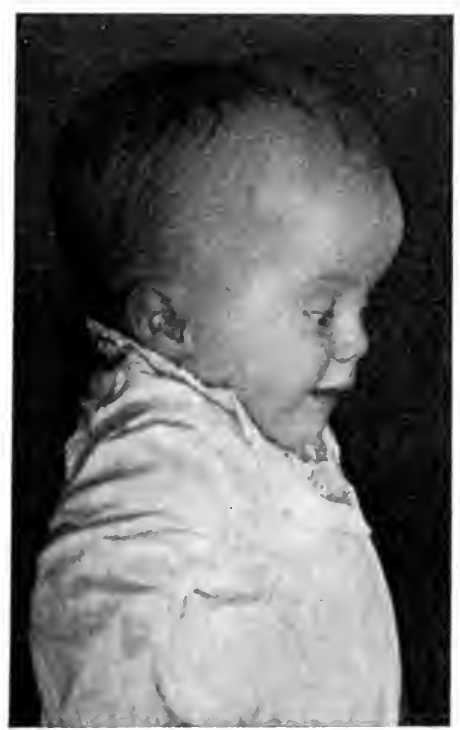

Fig. 53.-Hydrocephalus (side view) (Kerr).

Cerebral paralysis may occur after birth, in which case the same symptoms are found.

Erb's paralysis is a form of birth paralysis not due to a hemorrhage of the brain. It affects the upper portion of the arm, and is due to an injury around the shoulder-joint during birth. 
Apoplexy is the term applied to a hemorrhage in the brain. Thrombosis of the sinuses of the dura occurs at times, most frequently after an operation upon the mastoid cells.

Abscess and tumors are rare in childhood. They cause pressure symptoms. The most common tumors are tubercular in character.

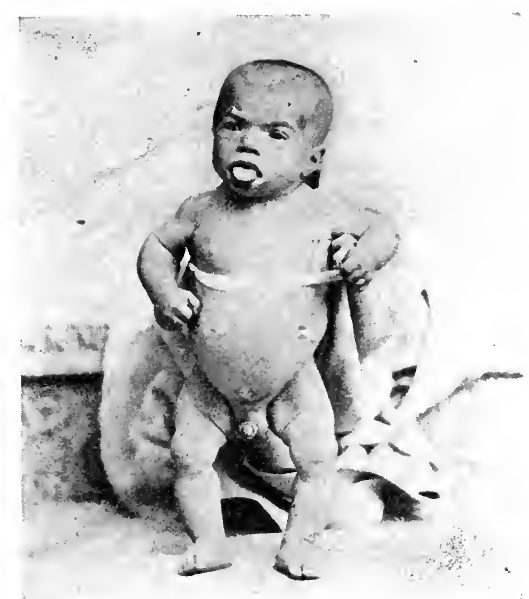

FIG. 54.-Sporadic cretin: before treatment. (From Osler, Sporadic Crelinism in America).

Hydrocephalus is a condition in which there is excessive fluid in the ventricles or in the arachnoid cavities. It gives to the head a peculiar shape. It is large and round, the sutures and fontanels are enlarged, the convolutions of the brain are flattened, and usually there is imbecility.

Cretinism is a congenital affection characterized by a lack of physical development due to an abnormal condition of the thyroid gland. The symptoms are myxedema (a waxy condition of the subcutaneous tissues), an abnormally large tongue, and idiocy or imbecility. 
The administration of thyroid gland extract to these cases causes a remarkable improvement.

Deaf-mutism is due to congenital or early loss of hearing. As the child has never heard spoken words he is unable to imitate the proper sounds.

Aphasia is a failure of word memory, an inability to utter words, to comprehend them, or to write them.

\section{EPILEPSY}

The disease apparently depends upon the instability of the motor centers, so that from trivial exciting causes violent discharges occur from time to time. The disease is divided into grand mal and petit mal.

Symptoms.-Grand Mal.-A peculiar sensation called an aura sweeps like a wave over the child. This is followed by unconsciousness and violent general convulsions, clonic in type. The child bites its tongue and froths at the mouth. The convulsion lasts for a few minutes and is followed by coma and later by automatism, in which the child performs certain automatic acts.

Convulsions occur at varying intervais, showing a tendency to increase in number and severity.

Petit mal is exhibited by momentary loss of consciousness with pallor, without convulsive movements.

Treatment. - For the Attack. - This consists in measures to prevent the children from injuring themselves. Something should be placed in the mouth to prevent biting of the tongue; further than this nothing can be done. If they should show any vicious traits after a convulsion, they should be carefully watched, as they are not responsible.

Prophylaxis. - Nitrite of amyl inhalations will at times ward off an attack. 


\section{HYSTERIA}

Hysteria is a functional disease of the nervous system associated with impaired will power and increased sensitiveness to impressions. Hysteric children are ill and should be treated accordingly. No doubt when our methods for examining the brain and spinal cord have improved, a definite lesion will be found. The impatience which some people show toward children suffering from hysteria is wrong.

Symptoms.-These are varied. They may be motor, sensory, and psychic.

Motor symptoms may be paralysis, usually hemiplegia, spasms, or convulsions. In hysteric convulsions the child is conscious, there is an absence of aura, the tongue is not bitten, the eyes are partially closed, and there is some emotion. The convulsions are tonic, or, if clonic, purposeful in character, and the seizures are of long duration.

Sensory symptoms may be complete loss of sensation in certain parts; the special senses may be impaired. The children may have hyperesthesia, clavus (the feeling of a nail being driven into the head), spinal irritations, globus hystericus (the sensation of a ball in the throat), and severe pain in the stomach.

Psychic Symptoms. - There is a great lack of will power, the children are easily moved to laughter or tears, and they have a fondness for sympathy. At times there is delirium and other mental conditions.

\section{CHOREA (ST. VITUS' DANCE)}

A nervous affection especially common in childhood and characterized by irregular movements which increase under excitement and cease during sleep. 
'The first manifestations are usually those of awkwardness in movement, and restlessness. These grow worse until the disease is fully advanced, when there are peculiar jerking, disorderly movements of the various members of the body or involvement of the whole body. The movement may be so marked that the child cannot use its arms in eating, it stumbles when walking, and grotesque expressions are produced from the involvement of the face. Involvement of the larynx causes stammering, involvement of the muscles of the pharynx causes choking fits and difficulty in swallowing, involvement of the tongue causes its withdrawal to be associated with an audible click. When the child's attention is called to the movements they invariably become worse. Frequently a heart murmur develops. The disease lasts from six to ten weeks.

Prognosis is good. Occasionally there are deaths from exhaustion.

\section{TETANY}

A tonic spasm of the muscles of the extremities. It

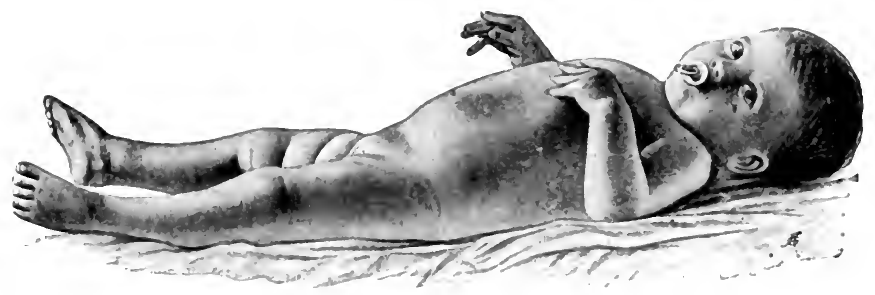

Fig. 55.-Persistent form of tetany in a girl a year and a half old. Tetanic coptractures of the arms and legs; hands in the "obstetric" position; feet in flantar flexion (Hecker, Trumpp, and Alt).

gives rise to a peculiar position of the hand called the obstetric hand, in which the fingers are slightly bent, the 
thumb held almost at a right angle across the palm, and the whole hand is slightly everted.

This spasm lasts for a variable length of time and can be excited by making pressure upon the nerve trunks and blood-vessels of the extremities (Trousseau's sign). The disease is usually associated with laryngismus stridulus and recovery nearly always takes place.

\section{HABIT SPASM}

A peculiar form of spasm caused by habitual grimaces or movements of the head, finally becoming uncontrollable. This condition is also called tic.

\section{NYSTAGMUS}

A constant movement of the eyes. It may be lateral, horizontal, or rotary.

\section{NODDING SPASM OR SPASMUS GYRANS}

A peculiar form of movement seen in children characterized by a continuous nodding of the head. It is associated with nystagmus. Recovery usually takes place in a month or two.

\section{TORTICOLLIS OR WRY-NECK}

This is a tonic spasm of the sternomastoid muscle. At times it is persistent.

Treatment.-If due to rheumatism the neck should be ironed with a hot iron, over a piece of flannel first laid on the skin for protection.

Disorders of Speech.-Stuttering or stammering and lisping are the most common. 
Disorders of sleep, such as night terrors, are common. The children awake from sleep with a cry of terror; they

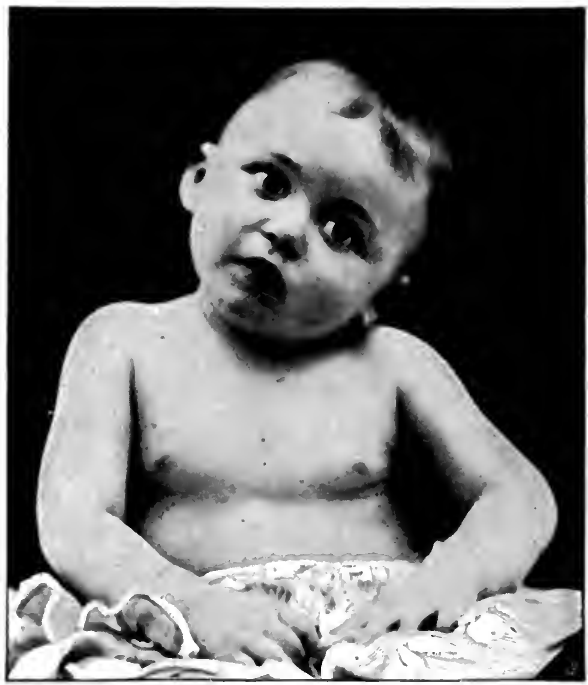

FIG. 56.-Torticollis in a child two years of age (Frühwald and Westcott).

fail to recognize those around them, and they exhibit symptoms of fright.

\section{Diseases of the Spinal Cord}

The functions of the different columns of the spinal cord are: 'The anterior and lateral columns-motor; the posterior columns-sensory; the anterior horns of gray matter-trophic and motor.

\section{MYELITIS}

Myleitis is an inflammaton of a segmant of the cord involving the anterior, lateral, and posterior columns and the gray matter. 
Symptoms.-At first there is irritation, producing pain and fever. The pain is a peculiar one called a girdle pain. The reflexes are increased; there are paresthesias and convulsive movements. Later, when the inflammatory product becomes sufficient to produce pressure, there is a loss of sensation instead of pain and paresthesias. The reflexes are lost, there is paralysis instead of convulsions, and there is degeneration of the muscles and bed-sores.

\section{SCLEROSIS}

Sclerosis is an atrophy of the structure of the part affected with an overgrowth of connective tissue. Sclerosis in the spinal cord is an atrophy of the nerve elements and an overgrowth of the neurilemma (the connective tissue of the cord).

Lateral sclerosis is the term applied when this degeneration attacks the lateral columns of the cord. This disease does not affect the sensory fibers; the main motor fibers which run through the anterior columns are intact and there are no trophic disturbances.

Symptoms.-Exaggerated knee-jerks, ankle-clonus, and a spastic gait, sometimes spoken of as scissors gait.

Acute Anterior Poliomyelitis or Infantile Paralysis. - This is an acute disease which occurs almost exclusively in young children, and is characterized by the destruction of nerve-cells in the brain and spinal cord, especially in the anterior horns of gray matter.

Since 1907 epidemics of infantile paralysis have been prevalent in Europe and the United States. Flexner and Lewis in their epoch-making studies having proved it to be an infectious and probably a contagious disease. The 
virus of infection most probably gains access to the central nervous system through absorption from the mucous membrane of the nose and throat, from whence it is carried by the lymphatics through the cribriform plate of the

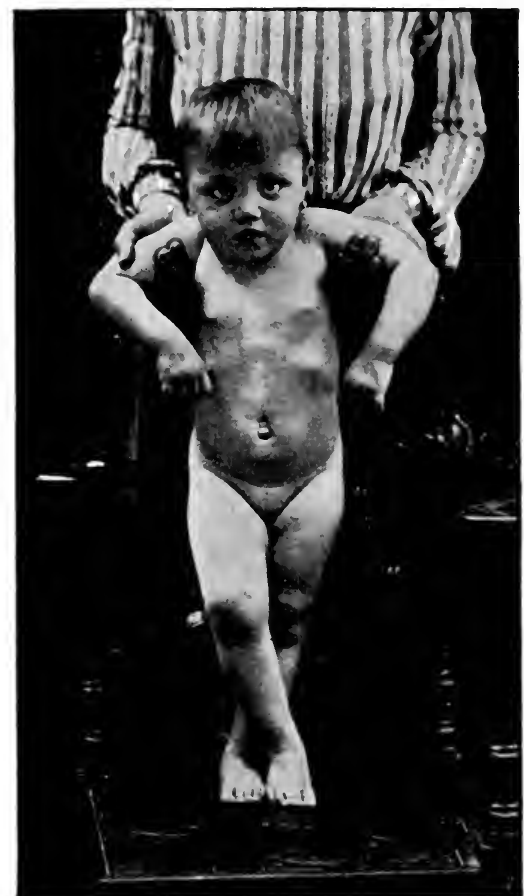

Fis. 57.-S(issors gait in a girl two years old (Frühwald and Westcott).

ethmoid bone directly into the cranial cavity. The poison is likewise thrown off from the same mucous membrane by a reversed process of elimination.

Symptoms. - The paralysis comes on very suddenly. The child goes to bed well and the following morning he 
cannot move his legs or, at times, his arms. Certain groups of muscles in the upper and lower extremities are involved, chiefly the latter. The paralysis at first is widespread, but tends to improve up to a certain point, where it remains stationary. The muscles affected atrophy, and the usefulness of the limb is obtained by an overdevelopment of other muscles which perform the function of the muscles which have been destroyed to a limited degree.

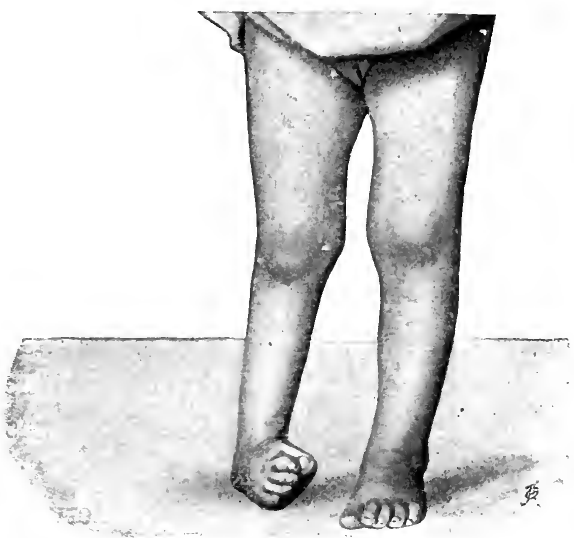

Fig. 58.-Spinal infantile paralysis in the stage of fully developed palsy. Three-yearold girl (F. Lange).

Treatment.-It is highly probable that in the near future a serum treatment will be perfected for this disease. The nose and throat, being the principal point of infection, should be thoroughly douched with antiseptic solutions containing hydrogen peroxid and menthol. This not only applies to the children attacked, but to all children when the disease is epidemic.

Syringomyelia is a disease of the spinal cord in which there is a cavity in the cord. 
Landry's paralysis is an ascending form of paralysis beginning in the legs and rapidly involving the entire body.

Friedreich's ataxia is a form of sclerosis of the spinal cord which develops in childhood and lasts from twenty to thirty years.

Atrophies of the muscles of different parts of the body are seen, due to disease of the spinal cord.

Pseudohypertrophy of the muscles is a condition in which the muscles are apparently enlarged, but actually are degenerating.

\section{Diseases of the Nerves}

NEURITIS

Neuritis is an inflammation of a nerve, and is characterized by pain and tenderness along the course of the nerve. It is associated with various forms of paresthesias. The part supplied by the nerve is at first hyperesthetic, later anesthetic. In severe inflammations paralysis of the part supplied by the nerve develops.

Sciatica is inflammation of the sciatic nerve characterized by the above symptoms along its course in the posterior part of the thigh. It is worse at night and at the approach of stormy weather.

Multiple neuritis is an inflammation of a number of nerves. The most common cause in childhood is diphtheria.

Symptoms.-There is pain over the deep nerve-trunks, paralysis, and wrist-drop and foot-drop due to paralysis of the extensor muscles.

Postdiphtheritic paralysis is a form of multiple neuritis occurring after diphtheria in which the muscles of 
swallowing are at first attacked, the other muscles of the throat and body being successively involved. Recovery usually follows. If the children die the cause of death is heart failure due to paralysis of the pneumogastric nerve (see page 296).

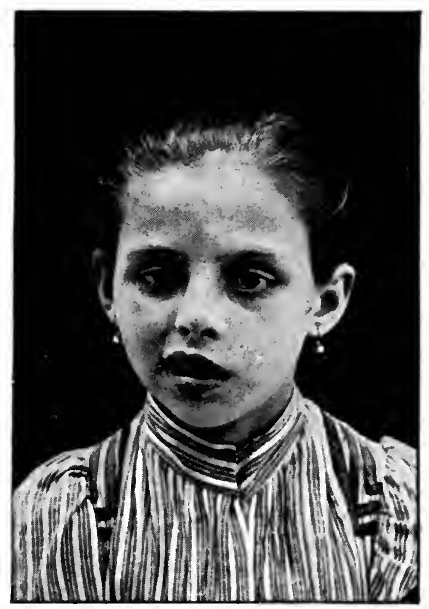

FIG. 59.-Left-sided facial or Bell's palsy of eight years' standing in a girl ten years of age (Frübwald and Westcott).

Bell's Palsy.-A paralysis of the muscles of expression (Fig. 59) affecting one side of the face and due to injury or disease of the seventh cranial nerve.

\section{NURSING IN NERVOUS DISEASES}

When symptoms of irritation of the central nervous system exist the child should be placed in a dark room, kept as quiet as possible, and the covers should be supported by a frame to prevent the discomfort of their weight. 
Scrupulous cleanliness must be employed to prevent bed-sores. The position of the child must be frequently changed, parts resting upon the bed must be protected, using air-cushions and water-beds for this purpose.

The symptoms developing in the course of nervous diseases must be accurately recorded, convulsions must be observed, and their starting-point, character, extent, and duration reported.

In lumbar puncture the fluid is collected in a sterile test-tube with an aseptic cotton stopper.

At times feeding must be administered by the medicine, dropper, gavage, or enema.

In chorea the extremities should be bandaged to protect them from chafing. Pull all sheets very tight and dispense with the "draw sheet." Remove all crumbs immediately and apply powder to bed frequently.

The temperature, pulse, and respirations should be taken three times a day. 


\section{CHAPTER X}

\section{DISEASES OF THE URINARY TRACT}

THE urinary tract consists of the kidneys, two in number, situated on the right and left side of the body beneath the edge of the ribs, posteriorly; the ureters, which run from the pelvis of the kidneys; the bladder; and the urethra.

\section{THE KIDNEYS}

Anatomy.-The kidney is divided into the cortical area, the medulla or pyramidal, and the pelvis.

The uriniferous tubules start in the glomeruli which are situated in the cortical area of the kidney.

The glomeruli are tufts of capillaries surrounded by a capsule. The epithelial cells lining the capsule and tubes abstract from the blood current the products which form the urine. The tubes run from the capsules and after pursuing a very tortuous course they empty at the apex of one of the Malpighian pyramids. It is in these tubules that the urinary products are formed. They are emptied from the mouths of the uriniferous tubules, at the apices of these pyramids, into the pelvis of the kidneys, and from here the urine passes through the ureters into the bladder and is voided through the urethra.

From these tortuous uriniferous tubules the tube-casts are formed. They are cylindric in shape and usually composed of the lining epithelium in various stages of 
degeneration. Their formation is something on this order: The lining epithelium of these tubes is the filter which extracts the uriniferous products from the blood. As long as this epithelial lining is intact the urine is normal; when there is congestion or inflammation of the kidneys the tubules shed this lining membrane. This appears in the urine as casts, and as the wall between the

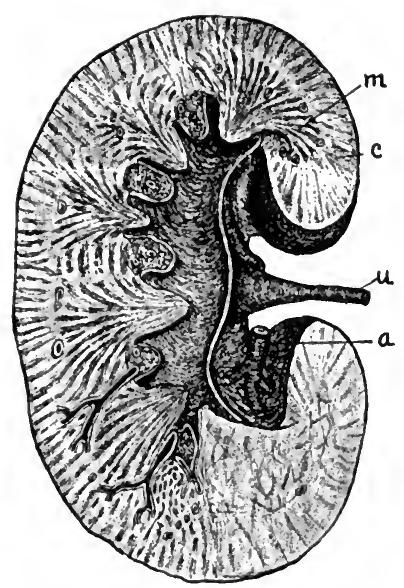

Fic. 6o.-A longitudinal section of the kidney. $a$, Renal artery; $c$, cortex; $m$, medulla; $u$, ureter (Leroy).

blood current and the tubules is destroyed there is an outflow of red blood-corpuscles and albumin. This is the condition seen in acute congestion, or acute nephritis, the name for inflammation of the kidneys. In chronic nephritis fatty casts are found.

Malformations of the kidneys are not common. Those seen occasionally are horseshoe kidney, where 
the two are fused, forming one large crescentic kidney; supernumerary ureters; floating kidney; and single kidney.

\section{THE URINE}

Normal urine is pale, amber colored, of acid reaction, and has a specific gravity of 1015 to 1025 .

Polyuria is the term applied to an increase in the quantity of the urine, and may be a temporary polyuria, such as is seen in excessive ingestion of fluids, the suppresison of perspiration, etc.

Permanent polyuria may result from such conditions as diabetes mellitus, diabetes insipidus, chronic interstitial nephritis, and amyloid kidney.

Anuria is the term applied to a diminution in the flow of urine. It is seen in the following conditions: Excessive secretion through other channels, such as profuse perspiration and diarrhea; in fever; passive renal congestion from obstructive disease of the heart, lung, or liver; organic obstruction in the urinary passages; in acute or chronic parenchymatous nephritis; from nervous causes such as hysteria; and in the reflex inhibition after abdominal injuries or operations.

Urea results from the perfect decomposition of the nitrogenous elements of food and tissue. It is, therefore, a normal constituent of the urine. In health the amount excreted varies greatly. Normal urine contains about 2 to $2 \frac{1}{2}$ per cent. of urea. When there is imperfect metamorphosis of tissues and nitrogeous food there is the formation of uric-acid crystals in the urine. When they are in excess the urine is heavy, dark in color, and on cooling throws down a brick-red deposit. When uric acid or urates are found in the urine it is termed lithuria. 
Glycosuria is the name applied to urine containing sugar. It is caused by diabetes mellitus and at times by chorea, tetanus, and functional nervous affections; indigestion of a large amount of saccharine material, lesions of the pancreas, liver, and base of the brain.

Albuminuria is the name applied to the presence of albumin in the urine. It is found in all forms of nephritis and in congestion of the kidneys, resulting from chronic disease of the heart, lung, or liver.

Cyclic Albuminuria.- The urine may be albuminous at certain times, as after meals, bathing, or rising in the morning.

Accidental albuminuria results from the admixture of albuminous substances with the urine, as pus and blood. It is found in certain nervous diseases, as epilepsy, tetanus, and injuries to the brain, extreme anemia, and the ingestion of large amounts of albuminous foods.

Hematuria.-Blood in the urine.

Hemoglobinuria.-Blood-pigment in the urine.

Choluria.-Bile in the urine.

Chyluria.-Chyle in the urine.

Pyuria.-Pus in the urine.

Indicanuria.-Indican in the urine. It is a symptom of chronic indigestion.

Method of Collecting Urine.-In males the penis can be placed in the neck of a bottle which lies between the thighs, and is held in position by a square of adhesive plaster, the center of which is pierced, making a hole large enough to grip the neck of the bottle.

In females a small pan placed under the buttocks will answer, or a bottle may be arranged as described above. If these methods fail, catheterize (see page 437). 
Average Daily Quantity of Urine in Health Age

Ounces

First twenty-four hours................ o to 2

Second twenty-four hours............... $\frac{1}{3}$ to 3

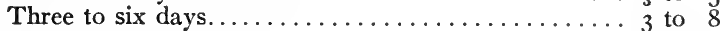

Seven days to two months.............. 5 to $\mathrm{r} 3$

Two to six months.................. 7 to I6

Six months to two years................. 8 to 20

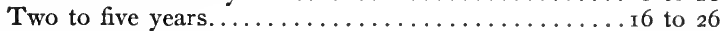

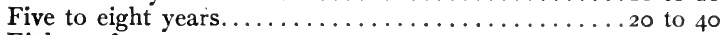

Eight to fourteen years.................. $3^{2}$ to 48

(Holt).

Examination of Urine.-The color is noted and any sediment is recorded. The acidity or alkalinity is determined.

The specific gravity is found by means of a urinometer. This is an instrument weighted with mercury and having a scale on the stem graduated from rooo up. By floating this instrument in a quantity of urine the specific gravity of the specimen can be read off. The point at which the top of the liquid is indicated on the scale is the proper specific gravity.

Albumin is tested for by boiling a small quantity of urine in a test tube. It should be clear urine, filtered if necessary. If albumin is present the urine will cloud when boiled, and if upon the addition of a few drops of acetic acid it does not clear, albumin is present. If it does clear the precipitate is composed of phosphates.

Heller's Test.-Another test used consists in placing a small amount of nitric acid in a test-tube and allowing the urine to run slowly down the sides of tube in such a manner that it will float on the surface of the nitric acid. If albumin is present there will be a white line at the point of contact of the two liquids. A brown line denotes uric acid. 
Sugar is tested for by Fehling's solution. This consists of two parts: One is bluish-green (composed of copper sulphate) and the other is white (composed of rochelle salts and caustic potash). Equal parts of each are added to four times their volume of water and the mixture boiled. If it retains its bluish color it is suitable for a test. A few drops of urine are added to the solution and boiled, and if sugar is present, a reddish-yellow precipitate is thrown down. The test is very accurate.

Microscopic examination is necessary to determine the presence or absence of casts, red blood-cells, white bloodcells, and epithelium.

Such substances as bile in the urine have special tests.

\section{DIABETES INSIPIDUS}

Diabetes insipidus is a chronic condition characterized by the excretion of large quantities of pale, limpid urine of low specific gravity and free from albumin and sugar.

In addition to the urinary symptoms there is an insatiable thirst, good appetite, a harsh, dry skin, a dry tongue, constipation, mental apathy, and emaciation.

\section{RENAL HYPEREMIA}

Active hyperemia is caused by arterial blood and is found in all acute congestions. It is due to exposure when the body is overheated. The same cause, aggravated, would cause acute nephritis.

Passive hyperemia is due to venous stasis and is found in all chronic congestions.

Acute Congestion.-Symptoms of acute congestion are pain over the loins; dark and scanty urine of high specific gravity, and perhaps containing a trace of albumin, a few hyaline casts, and some free blood. 
Passive congestion of the kidneys is caused by conditions which obstruct the general circulation, such as chronic disease of the heart, liver, and lung; pressure of tumors upon the renal veins, and, rarely, thrombosis of the renal veins. The kidney is swollen, and in protracted cases becomes hard from an overgrowth of connective tissue.

Symptoms. - There is a sensation of weight over the loins; the urine is usually diminished in quantity; rarely increased. Free blood, a trace of albumin, and occasionally a few hyaline casts are found.

\section{UREMIA}

Uremia is the name applied to a group of symptoms which result from the retention of toxic materials in the blood which should have been eliminated by the kidneys.

Sym ptoms. - The disease may develop slowly or abruptly and may manifest any of the following phenomena: headache, vertigo, delirium, epileptiform convulsions, coma, sudden blindness (unassociated with any retinal change), and transient paralysis from congestion of the brain or cord.

Pulmonary symptoms are dyspnea (uremic asthma), and Cheyne-Stokes respiration.

Abdominal symptoms are hiccough, obstinate vomiting, and purging.

General Symptoms.-The skin is dry, the breath has a urinous odor, the urine is scanty and deficient in urea. The pulse is slow and full, the temperature is subnormal, but during a convulsion the temperature may rise and the pulse become rapid and feeble.

Prognosis.-Grave, but always guarded, for recovery 
is possible after the most serious complications and manifestations.

Trealment.- The object of the treatment is to eliminate the poison as rapidly as possible. To accomplish this the physician takes advantage of every possible excretory function. He promotes catharsis (purging) and free diaphoresis (sweating). In some cases bleeding and the intravenous injection of normal salt solution is practised. Convulsions may be controlled by inhalations of chloroform. Morphin should be used with great care, as it frequently aggravates the case.

\section{NEPHRITIS}

Acute nephritis is an acute inflammatory disease involving more or less of the whole kidney, but it especially affects the epithelium of the tubes and glomeruli.

Other names given to this condition are acute Bright's disease and acute parenchymatous nephritis.

Etiology.-Exposure to cold and wet, the specific fevers, especially scarlet fever, and certain poisons.

Pathology. - The kidney is swollen, the capsule is nonadherent. At first the organ is bright red in color, but it soon becomes pale and mottled in appearance.

Histology. - The epithelium of the tubes and of the glomeruli is the seat of the cloudy swelling and, later, of fatty degeneration. Desquamated epithelium, blood corpuscles, and an albuminous exudate block up the tubules.

Symptoms.-In some cases moderate fever with its associated phenomena, dull lumbar pains, nausea and vomiting, dropsy, beginning in the face and becoming general, and rapid anemia. Uremic symptoms may develop at any time. The urine is scanty and at times 
suppressed. It is smoky in appearance, of high specific gravity, rich in albumin, and throws down a heavy sediment which contains hyaline, blood, and epithelial casts and free blood and epithelial cells. As the general symptoms are often slight, the diagnosis of the condition must rest upon the urinary analysis.

Prognosis.-Guardedly favorable. It may kill by exhaustion, uremia, or dropsy, and it may become chronic.

Treatment.- The disease demands absolute rest in bed until the albumin has entirely disappeared from the urine. Milk is the best food, although butter-milk, gruels, and light broths are admissible. The free use of water should be encouraged. Free action of the skin is secured by means of vapor baths and the bowels are kept loose by concentrated saline draughts, as Rochelle and Epsom salts. Uremia will call for its appropriate treatment. Marked effusions in the serous cavities will sometimes demand aspiration. Convalescence should be protracted.

Chronic parenchymatous nephritis may result from acute nephritis or it may be chronic from the beginning. Congestion from heart disease is the usual cause.

Pathology.-In the first stage the kidney is large and pale yellow in color, the pallor depending upon the anemia and the fatty degeneration. The tubes are filled with fatty epithelium and casts, and there is always some overgrowth of the interstitial connective tissue.

In the second stage the organ is small, pale in color, its surface rough, and its capsule somewhat adherent. The reduced size depends upon the destruction of the renal epithelium and the contraction of the overgrown connective tissue.

Symptoms.-As the disease usually begins as a chronic 
affection the following symptoms slowly make themselves manifest: progressive loss of flesh and strength, marked anemia, gastro-intestinal disturbances, dropsy, often first noted in the face on arising in the morning; increased arterial tension, some hypertrophy of the left ventricle of the heart so that the second sound at the aortic cartilage is accentuated. Uremic symptoms may develop at any time.

The urine is usually diminished although it is often normal in color and appearance. It is highly albuminous and throws down an abundant sediment which contains hyaline, fatty, and granular casts, and fatty epithelium. The process is a chronic one and the epithelium and the casts have undergone fatty degeneration.

Complications.-These are numerous and often suggest the diagnosis. The most common are uremia, extensive dropsy into the tissues or serous cavities, valvular heart disease, albuminuric retinitis (an eye condition interfering with vision), and acute exacerbations (an acute parenchymatous nephritis occurring during the course of the chronic attack).

Treatment.-The treatment is largely hygienic and dietetic. Residence in a dry, warm, and equable climate may prolong life or affect a cure. Rest is an essential element in the treatment. The underclothing should be woolen or silk in order to keep the skin constantly active. The diet should be non-nitrogenous and in severe cases an absolute milk diet may be of extreme value. The bowels should be kept active by mineral waters.

Chronic Interstitial Nephritis.- This disease, rare in childhood, is a chronic inflammatory condition of the kidney characterized by a reduction in its size due to an 
overgrowth and a subsequent contraction of its connectivetissue elements. It may be associated with general arteriosclerosis and cardiac hypertrophy.

Etiology.-The disease may be secondary to parenchymatous nephritis or it may result from the chronic congestion of chronic heart disease; but it generally arises as a primary condition and results from the causes which predispose to sclerosis in other organs.

Pathology.-The kidneys are small and red in color. The surface is granular and the capsules adherent. Small cysts are often present. The microscope shows a great overgrowth of connective tissue which has contracted, narrowing the lumen of the tubules and interfering with the nutrition of the epithelium, and as a result the epithelium may show fatty degeneration with desquamation. The arteries throughout the body may show sclerosis, and from the resistance thus offered hypertrophy of the heart results.

Symptoms.-A slow loss of flesh and strength with progressive anemia. Gastric disturbances are very common. The arteries may be rigid and the pulse is of high tension, so that the second sound of the heart is accentuated at the aortic cartilage. Palpitation of the heart is often noted. Dyspnea is a prominent symptom and may result from heart weakness, uremia, or edema of the lungs. Headache, vertigo, and insomnia often result from disturbed circulation and dimness of vision from albuminuric retinitis. Dropsy is often absent or is slight and appears late in the disease.

The urine is increased in quantity, pale in color, and of low specific gravity, 1005 to roro; it contains but a trace of albumin and a few narrow hyaline casts. 
Diagnosis.-The difference between chronic interstitial nephritis and chronic parenchymatous nephritis is that in the latter the urine is rich in albumin and tube-casts and the disease is seen in earlier life; it lacks much arterial change and produces considerable dropsy.

Chronic interstitial nephritis is so insidious that it is scarcely ever discovered except by accident. Either it is discovered in the routine examination or, as frequently happens, not until uremia develops, when often it is too late to do anything.

The treatment is practically the same as in chronic parenchymatous nephritis.

\section{AMYLOID KIDNEY}

Amyloid kidney is a name given to the large white kidney found after prolonged suppuration, particularly bone disease; in tuberculosis, syphilis, and malarial cachexia.

The urine is increased, there is considerable albumin, and wide hyaline and granular casts.

\section{RENAL CALCULUS}

Renal calculus is a precipitated urinary concretion found in the kidneys. The stone may lie latent indefinitely or it may pass out with or without symptoms of colic. It may obstruct the ureter or excite inflammation of the kidney or even abscess.

Symptoms of Renal Colic.-Sudden onset with sharp pain, starting in the back and radiating down the ureter into the penis, testicle, or thigh. The symptoms of intense pain are present: pallor, cold sweats, weak pulse, and 
reflex vomiting. The urine subsequently passed may contain the stone, or, as a result of irritation, pus, blood, and desquamated epithelium. An attack may last from a few moments to several hours.

Treatment.-The physician will probably use hypodermics of morphin and atropin, and inhalations of chloroform if necessary. The free use of water should be encouraged, and hot applications placed over the loins.

\section{PYELITIS}

Pyelitis is the name given to an inflammation of the pelvis of the kidney.

\section{HYDRONEPHROSIS}

This is a dilatation of the pelvis of the kidney with an accumulation of watery fluid resulting from obstruction. It is caused by strictures, tumors, and impacted stones.

\section{FLOATING KIDNEY}

This condition is rare in childhood. The kidney is distinctly mobile, due to a relaxation of the tissues which surround it. The right kidney is the one usually affected and may be found in any part of the abdomen.

\section{TUBERCULOSIS OF THE KIDNEY}

This condition is usually secondary to tuberculosis elsewhere, although it may be primary. It is always grave; the patients may live from a few months to three years. 


\section{SARCOMA OF THE KIDNEY}

Sarcoma of the kidney is a malignant tumor of the kidney which is sometimes seen in children. The organ affected is removed at times by surgical means. It is ultimately fatal.

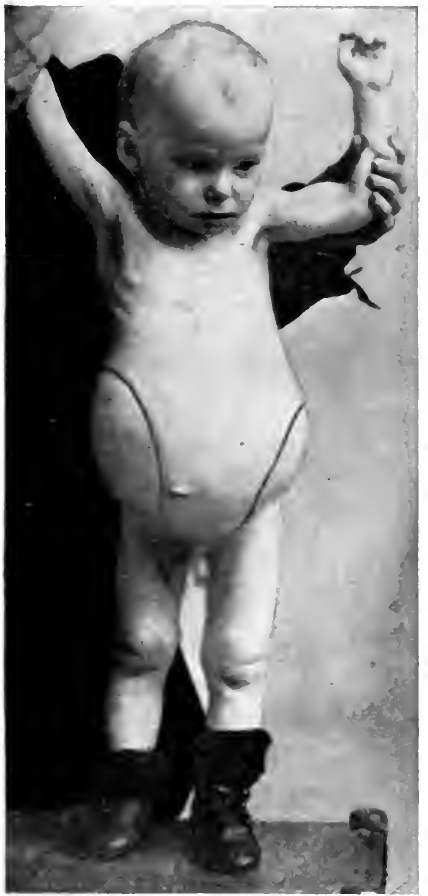

Fig. 61.-Sarcoma of both kidneys in a male child two years of age. The tumors are here outlined to show their extent (Napier).

\section{NURSING IN KIDNEY DISEASES}

In nephritis the room should be warm to facilitate the action of the skin.

Vapor baths are necessary at times (see page 403). 
Woolen underwear should be worn to stimulate perspiration.

The child should lie between blankets while in bed.

A specimen of the urine should be saved daily, unless otherwise ordered. The total quantity of urine passed in twenty-four hours should be measured.

The feeding should be carried out strictly in accordance with instructions.

Any symptoms of uremia, such as convulsive movements, intense headache, or stupor should be immediately reported.

The temperature, pulse, and respirations should be taken at least three times a day.

\section{THE BLADDER}

Enuresis is the inability of a child to hold its urine. In infancy urination is a reflex act beyond the control of the will. After the second year, and often before this age, a child should be able to retain the urine until a suitable time and place for voiding is reached. After two years involuntary urination is spoken of as enuresis. If it occurs at night it is called nocturnal, and if in the daytime, diurnal. In some cases it occurs both during the day and at night.

The children suffering from enuresis are usually anemic, underdeveloped, and of a very nervous disposition.

Treatment.-Plenty of sleep and a diet of milk, vegetables, fruits, meats, and cereals should be adhered to. Often a case of nocturnal enuresis can be controlled by awakening a child at eleven or twelve o'clock at night and making him void urine. Another way is to get the child to hold urine as long as possible in the day-time. This accustoms the sphincter to retain the urine. Medical 
treatment is usually necessary. Circumcision at times relieves, if there is a long foreskin.

Vesical spasm is a frequent and painful micturition usually due to highly acid urine.

Vesical calculus is a stone in the bladder. This gives rise to painful micturition and straining, which at times causes prolapse of the rectum. Occasionally there is sudden stoppage of the flow and pain in the end of the penis.

\section{MALFORMATIONS OF THE GENITAL TRACT}

Phimosis is a narrowing of the prepuce or foreskin so that it cannot be retracted. This condition gives rise to irritation from accumulation of smegma beneath the skin, obstruction to the flow of urine, enuresis, and masturbation.

Treatment.-Circumcision should be done in all cases of phimosis. If the child is vigorous, two years of age is the best time for an operation.

Hypospadias. - A malformation of the penis in which the urethra is not continued to the end of the glans, the orifice being on the under surface of the penis.

Epispadias.-The opposite of hypospadias, the orifice being on the upper surface of the penis.

Exstrophy of the Bladder.-A failure on the part of nature to complete the abdominal wall over the bladder. The bladder is in sight and often fissured so that the urine discharges from it.

Undescended Testicle.-Before birth the testicles lie beneath the kidneys. They descend into the scrotum during the ninth month of pregnancy. At times they fail to descend. An undescended testicle usually lies in the inguinal canal. Serious symptoms rarely attend this deformity. 


\section{DISEASES OF THE MALE GENITALS}

Balanitis.-Inflammation of the prepuce or foreskin. Treatment.-Wash with I to 5000 solution of bichlorid of mercury, syringing beneath the foreskin.

Urethritis.-Inflammation of the urethra. The chief symptoms are painful urination and a discharge. This discharge may be a simple catarrhal discharge or it may be gonorrheal. If the latter, gonorrheal infection of the eyes (gonorrheal conjunctivitis) must be very carefully guarded against. The parts should be covered and kept clean.

Hydrocele. - This is a collection of serous fluid in the testicle or along the inguinal canal.

\section{DISEASES OF THE FEMALE GENITAL TRACT}

Vaginitis.-An inflammation of the vagina. The chief symptom is a yellowish discharge from the vagina. It may be catarrhal or gonorrheal. The gonorrheal form is highly contagious and very obstinate. It may cause gonorrheal conjunctivitis (inflammation of eye) with a loss of sight.

Girls suffering from vaginitis should not be allowed to associate with other children and their towels and diapers should be sterilized. The parts should be kept scrupulously clean and well protected, and frequent douches of a solution of bichlorid of mercury, I to I0,000; potassium permanganate, I to $10, \infty 00$; saturated solution of boric acid, or normal salt solution administered (see page 420).

\section{NURSING IN DISEASES OF THE GENITAL TRACT}

Whenever a discharge exists from the male or female generative organs the nurse should thoroughly sterilize 
her hands after cleansing the parts so as to prevent any infection of her eyes. If possible, rubber gloves should be worn.

To prevent the spread of vaginitis either in a hospital or in a private family the greatest precautions must be taken. A child affected should have a separate bed-pan, douche-bag, thermometer, eating utensils, playthings, etc. If possible, all articles which come in contact with the discharge should be sterilized after using. All linen should be boiled, and all soiled articles, such as cotton, etc., should be soaked in carbolic acid, I to 20 , before disposal. A pad should be worn, which should be immediately burnt when discarded.

Vaginal suppositories are used in treating this disease by many physicians. They must be carefully inserted. 


\section{CHAPTER XI}

\section{DISEASES OF THE EYE, EAR, SKIN, AND GLANDULAR SYSTEM}

\section{THE EYE}

Anatomy.-The eye is a globe composed of three coats: the sclerotic, the choroid, and the retina. The retina is the sensitive coat; it receives the impressions of the objects seen and transfers them through the optic nerve to the brain.

The anterior portion of the eyeball is composed of the cornea, a transparent membrane.

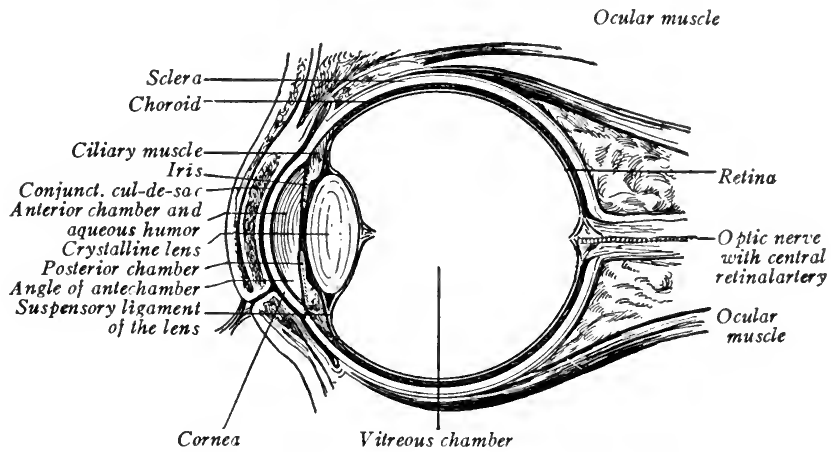

Fig. 62.-Vertical section through the eyeball and eyelids (Pyle).

The iris is the curtain behind the cornea which shuts out the unnecessary rays of light. It has an opening in its center called the pupil.

The eyeball is moved horizontally and vertically by the 
superior, inferior, internal, and external recti muscles; in the oblique directions by the superior and inferior oblique muscles.

The conjunctiva covers the eyeball and lines the lid; it is a mucous membrane.

The lacrimal apparatus consists of the lacrimal gland which secretes the tears. The tears are poured over the eyeball from a duct at the inner canthus, and drain through a duct leading into the nose.

The Meibomian glands are small glands in the upper lids which secrete a lubricating fluid.

Definitions.-Photophobia.-Intolerance to light.

Lacrimation.-Watering of the eye.

Conjunctivitis.-An inflammation of the lining membrane of the lids and the covering of the eyeball.

Keratitis.-An inflammation of the cornea.

Ophthalmia.-An inflammation of the eye.

Iritis.-An inflammation of the iris.

Strabismus (Cross-eyes).-A paralysis of one of the rectus muscles.

Synechia.-Adhesion of the iris to the cornea.

Hordeolum.-A stye; a cyst of a Meibomian gland.

Accommodation. - The power of the lens to change its form and shape so that objects nearby may be seen as readily and distinctly as those at a distance.

Hypermetropia.-Far-sightedness, as the eyeball is too short.

Myopia.-Near-sightedness. The eyeball is too long. Astigmatism.-A flattening of the convexity of a portion of the cornea or lens which blurs the vision.

Stenosis of the Lacrimal Duct.-A blocking up of the passage leading from the eye to the nose. 
Malformations.-Anomalies of the eyes consist of flecks upon the iris, cross-eyes, congenital cataract, and albinism.

Care of the Eyes.-Immediately after birth the eyes should be washed with a saturated solution of boric acid. This should be continued during the first week as a part of the daily bath. If the mother has had any vaginal discharge previous to the birth of the child, a drop of a

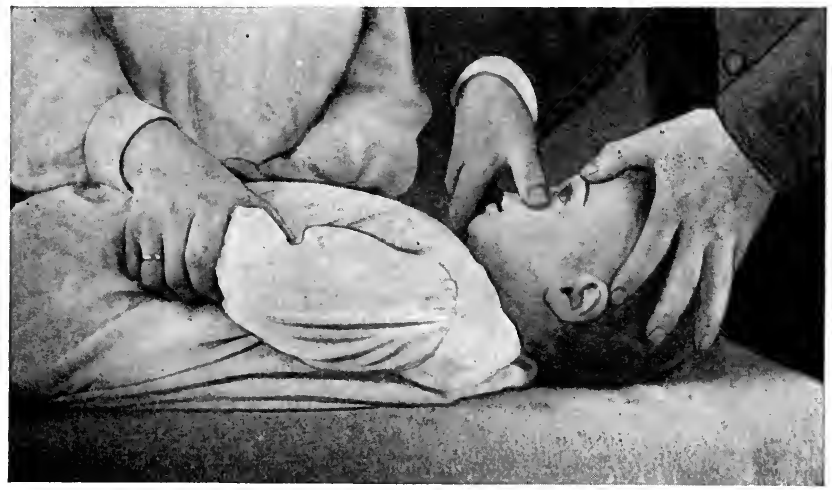

Fic. 63.-Examination of the eye. If the patient te a rebellious infant, perfect control may be secured if he is held firmly upon the nurse's lap, the child's head being steadied between the knees of the examiner while the examination is made (Kerr).

2 per cent. solution of nitrate of silver should be placed in each eye immediately after birth (Credé's method). This prevents ophthalmia neonatorum. For the method of syringing the eyes, see page 409 .

The newborn should be protected from too strong a light as it injures the sight; a dark room is the best.

Objects are recognized by the infant when about five months of age.

Ophthalmia neonatorum is an inflammation of the 
conjunctiva seen in the newborn. It is due to gonorrhea and is characterized by a purulent discharge from the eye. It very often causes blindness.

Conjunctivitis or Pink-eye.-An inflammation of the lining membrane of the eyelids and covering of the eyeball.

Symptoms.-Pain in the eye, lacrimation, photophobia, and a discharge which may be catarrhal or purulent. The disease may be acute or chronic.

Foreign bodies in the eye can be removed by turning the upper lid, if they are not visible. The lid can be turned by placing a card or match-stick at the center of the upper lid and pulling the anterior portion upward

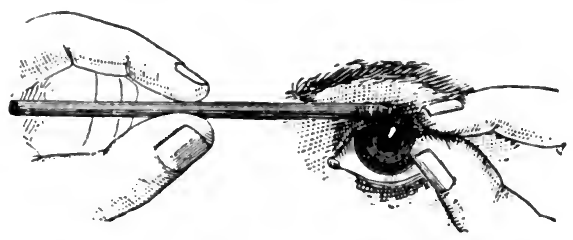

Fig. 64.-Method of everting the upper eyelid (J. P. C. Griffith).

by means of the eyelashes (see Fig. 64). A pledget of cotton will remove the speck.

Keratitis.-An inflammation of the cornea. It is civided into phlyctenular, ulcerative, and interstitial keratitis.

Symploms.-Pain, lacrimation, and photophobia. In the phlyctenular variety small vesicles appear upon the cornea which may rupture and form shallow ulcers. Scars result from healed ulcers. In the interstitial form the inflammation is not upon the surface, but within the structure of the cornea. This form of the disease is due to hereditary syphilis. 
Nystagmus consists of vibratory movements of the eyes, horizontally, vertically, or rotary.

Nursing of Diseases of the Eye.-When a discharge from the eye exists the lids should be held open and the conjunctiva syringed (see page 409) with a saturated solution of boric acid, repeated often enough to prevent any of the purulent matter from remaining in apposition to the inflamed surfaces for any length of time. The applications used are dropped in the eyes from a medicinedropper. Cold and hot compresses are employed to combat inflammation and the discharge.

Cold compresses are prepared by cutting small disks out of muslin, lint, or gauze and allowing them to lie on a piece of ice until cold. They are then laid over the eyelids. The compress must be changed every two minutes because it will not remain cold for a longer period.

Hot compresses are prepared in the same way, hot water being used instead of ice.

Children suffering from inflammation of the eyes should be kept in a dark room.

The nurse should always wash her hands in an antiseptic solution after treating ophthalmia, else she may infect her own eyes.

\section{THE EAR}

Anatomy.-The ear is divided into three divisions: the external or pinna, the middle ear, and the internal ear.

The external ear or pinna is composed of cartilage, which is so arranged that it collects the air waves, and the auditory canal, which is about one inch long.

At the internal end of the external auditory canal is the drum, or tympanic membrane, a small membrane about a half inch in diameter. 
The middle ear contains three bones: the malleolus, the incus, and the stapes. The malleolus is attached to the drum and articulates with the incus, which in turn articulates with the stapes. They are so arranged as to form a system of communication between the drum and the inner ear, by means of which the vibrations of the drum are transmitted to the inner ear.

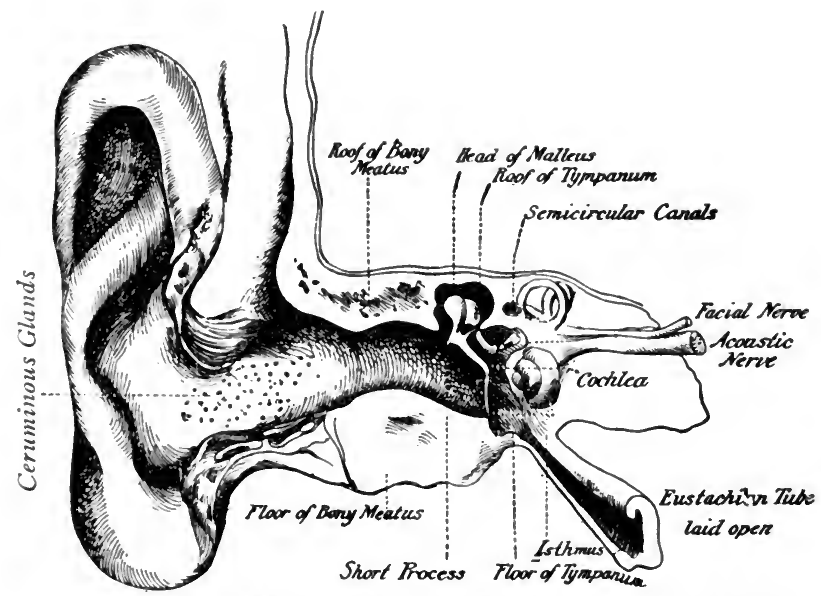

Fig. 65.-Frontal section of the organ of hearing (modified from Politzer).

The Eustachian tube connects the middle ear with the pharynx. It is through this duct that inflammation extends from the pharynx and causes middle-ear disease (otitis media).

The internal ear contains the organ of hearing called the cochlea. It is so arranged that the vibrations of the drum are transmitted to the terminal filaments of the auditory nerve.

The semicircular canals maintain the equilibrium of the 
body. Any disease or injury to them causes intense dizziness.

The mastoid cells are in the mastoid process of the temporal bone. They connect with the middle ear.

Definitions.-Impacted Cerumen.-The term given to large quantities of wax in the external auditory canal.

Furuncle of Canal.-A small abscess of the auditory canal.

Puncture of Drum.-A rupture of the drum or tympanic membrane.

Otitis Media.-Inflammation of the middle ear. It may be catarrhal or purulent. Acute and chronic varieties are seen.

Acute Otitis Media (Earache).--This is usually caused by an extension of inflammation from the pharynx along the Eustachian tube. It follows catarrh of the nose and throat, measles, scarlet fever, whooping-cough, and dentition.

Symptoms.-There is pain in the ear with congestion of the drum and fever, from $99^{\circ} \mathrm{F}$. to $102^{\circ} \mathrm{F}$. The child seems ill without any definite signs of the seat of the lesion. In a great number of cases at the end of twenty-four or forty-eight hours there will be a discharge from the ear. It may be catarrhal or purulent in character. The intense pain improves after the appearance of the discharge, and the condition gradually improves. The discharge is not present without a rupture of the drum. Rupture of the drum does not interfere with hearing.

In chronic otitis media the discharge is the characteristic symptom. It may persist for years. The purulent form has a disagreeable odor.

Mastoiditis is an inflammation of the mastoid cells. It is caused by an extension of the inflammation from the middle ear. 
Symptoms. - The characteristic symptoms are fever, $103^{\circ} \mathrm{F}$. to $104^{\circ} \mathrm{F}$, with swelling and tenderness behind the ear. An operation is demanded in the case of mastoid abscesses.

The dangers of mastoiditis are an abscess of the brain and septic thrombosis of the sinus of the brain and jugular

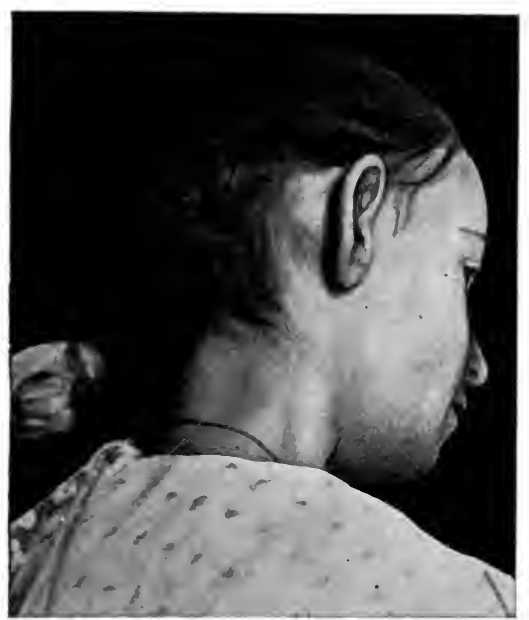

Fig. 66.-Mastcid abscess. The characteristic manner in which the external ear is pushed forward by the abscess is here well shown (Kerr).

veins. At times during the operation the facial nerve is injured, giving rise to Bell's palsy.

Nursing and Treatment of Ear Diseases.-The methods employed are used to keep the ear clean, dry up the discharge, and treat the inflammation. Hot water, II $5^{\circ} \mathrm{F}$, is the best agent to use in syringing the ear (for method, see page 410). It not only thoroughly cleanses but it reduces congestion. Nothing else should be used to remove foreign bodies or secretions from the ear. Syringing will be sufficient for all emergencies. 
Probing with instruments is dangerous. Powder of some form is used to dry up the discharge.

The throat is treated by local applications to cure the primary inflammation or congestion, and a Politzer bag is used to open the Eustachian tubes. This is accomplished by closing the mouth and nostril, the nozzle of the apparatus being inserted into the other nostril. As the patient swallows, the end of the Eustachian tube is opened, the air is forced from the bag into the nose, and it then passes through the Eustachian tube into the middle ear. At times it is necessary for the surgeon to lance the eardrum to relieve pain and allow the discharge to escape.

When shaving the head for a mastoid operation in girls leave a lock of hair in front which can later be drawn down over the scar. At the time of operation it can be retained beneath adhesive plaster. If the physician permits, a shampoo before the operation will afford a great deal of comfort during the weeks the bandage is worn.

\section{SKIN DISEASES}

Anatomy.-The skin is composed of three layers: the epidermis, the true skin, and the subcutaneous or supporting tissue.

The epidermis is the external layer and is without blood-vessels or nerves. It acts as a protecting membrane.

The true skin lies beneath the epidermis. It is exceedingly vascular and contains a meshwork of nerve filaments throughout its extent. The hair and sweat follicles are situated in the true skin.

Definitions.-Macules.-Small discolored areas of the skin without elevation.

Papules.-Solid elevations of the skin, varying in size from a pin-head to a pea. 
Vesicles.-Elevations containing a clear or opaque fluid. Blebs.-Large vesicles.

Pustules.-Elevations containing pus.

Scales.-Dry exfoliations of the epidermis.

Crusts.-Brownish or yellowish masses of dried exudation.

Erythema.-A reddish blush to the skin.

Miliaria (prickly heat) is caused by a blocking of the ducts leading from the sweat-glands. It produces small vesicles at the orifices of these ducts.

Treatment.-Keep the bowels loose, apply a good toilet powder, and prevent irritation by placing silk or linen next to the skin. A saturated solution of bicarbonate of soda, frequently applied, will give much relief.

Seborrhea is a disease usually involving the scalp; at times it is universal. It is characterized by the formation of dirty yellow crusts. The crusts should be removed by washing the scalp with warm water and soap and an ointment should be applied.

Eczema is an inflammation of the true skin.

It is caused by chapping of the skin surface from cold or discharges, such as running ears or coryza, and, reflexly, from the gastro-intestinal tract.

Symptoms.-Intense burning and itching of the skin with redness and weeping of the surface affected.

There are several varieties of eczema:

Eczema vesiculosa; principal lesions are vesicles.

Eczema papulosa; principal lesions are papules.

Eczema pustulosa; principal lesions are pustules.

Eczema rubrum, a raw weeping surface.

Eczema intertrigo, caused by two moist surfaces rubbing together, such as the thighs.

Treatment.-In eczema intertrigo bathe parts only once 
a day with water; for all other cleansing processes use olive oil and dust parts with a ro per cent. powder of balsam of Peru made with stearate of zinc.

In the other forms of eczema the crusts must be removed as soon as they form, and the ointment thoroughly applied.

To prevent children from scratching the inflamed skin pasteboard cuffs, well padded, are used. These are made

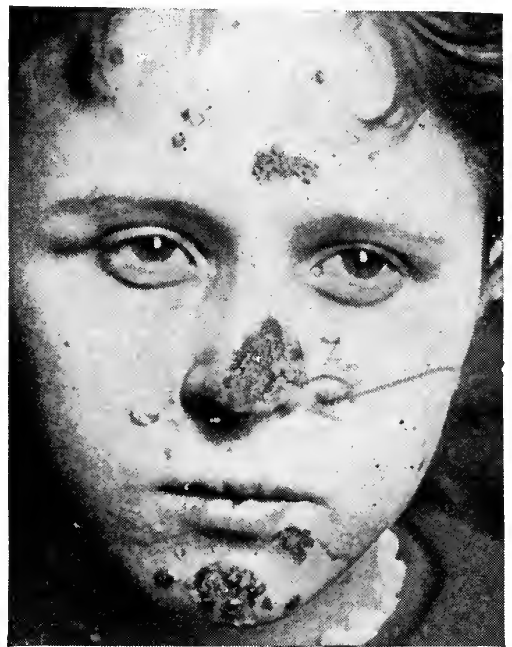

F1G. 67.-Impetigo contagiosa (of one week's duration) in a girl ten years of age, crusting stage already reached; on chin and nose lesions have coalesced (Stelwagon).

out of stiff pasteboard cut long enough to extend from the armpit to the wrist and wide enough to encircle the arm. They are held in place by a bandage. This prevents the bending of the elbow and renders it impossible for the child to scratch above the knees (see page 434 ).

Furunculosis is characterized by the formation of numerous. small abscesses or boils. 
Onychia is the name given to an inflammation at the root of the nail.

Impetigo contagiosa is a contagious skin disease often transmitted through towels, and is characterized by several large, flat pustules which break early and form crusts. The disease is usually seen on the face.

Urticaria or hives is characterized by red elevations which suddenly appear and vary from the size of a pea to that of a hand. They may be multiple. They itch - intensely and are soon covered with scratch marks from the finger-nails. They last from one to two hours. Repeated attacks are common.

Treatment.-Apply some soothing lotion and give a laxative.

Scabies, or the Itch.-A disease caused by a small "itch mite" or parasite buried under the epidermis. It is most often found between the fingers and the toes. The disease is characterized by intense itching.

Tinea Circinata.-A ring-worm of the skin, characterized by a circular irritation of the skin which fades toward the center.

Tinea Tonsurans. - A ring-worm of the scalp.

Lupus vulgaris is tuberculosis of the skin.

Nevus or Birth-mark.-A collection of blood-vessels in the skin. They are characterized by a raised purplish area. They should be excised if they cause disfiguration.

Pediculosis Capitis, or Lice.-Small parasites infesting the hair.

Pediculosis Pubis.--Small parasites infesting pubic hair.

Nursing.-To obtain results in skin diseases it is necessary to be patient and to apply the remedies thoroughly. 
DISEASES OF EYE, EAR, SKIN, AND GLANDS 239

All crusts should be immediately removed and the children prevented from scratching themselves. This may be accomplished by the use of cuffs (see page 433), or by tying the children down by the jacket (see page 432),

Impetigo, scabies, tinea circinata, and tinea tonsurans are contagious; therefore, antiseptic precautions must be taken after treating such cases.

\section{DISEASES OF THE GLANDULAR SYSTEM}

Anatomy.-The lymphatic glands are arranged in

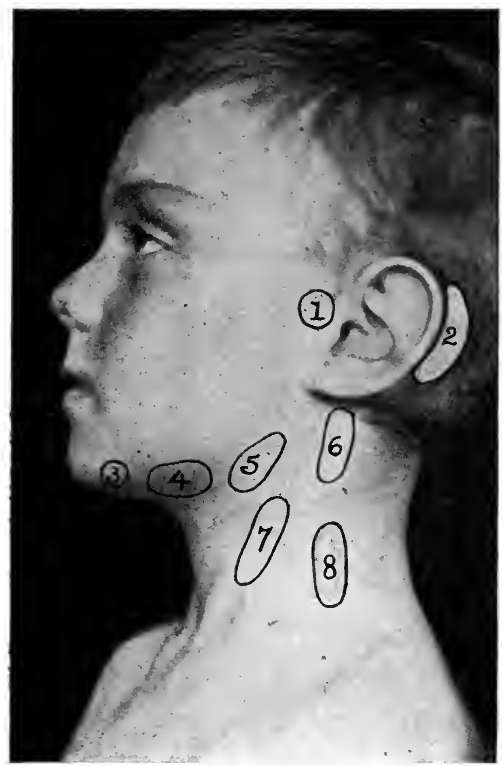

Fig. 68.-Location of lymphatic glands of face and neck (Kerr).

chains. They are situated along the lymphatic system. Their function is to act as filters, and keep poisonous 
substances and bacteria from entering the general circulation. The most important chains of the lymphatic gland in the body are the cervical, axillary, inguinal, bronchial, and mesenteric. They are located in the neck, armpit, groin, back of the bifurcation of the bronchi, and in the posterior part of the abdomen, respectively.

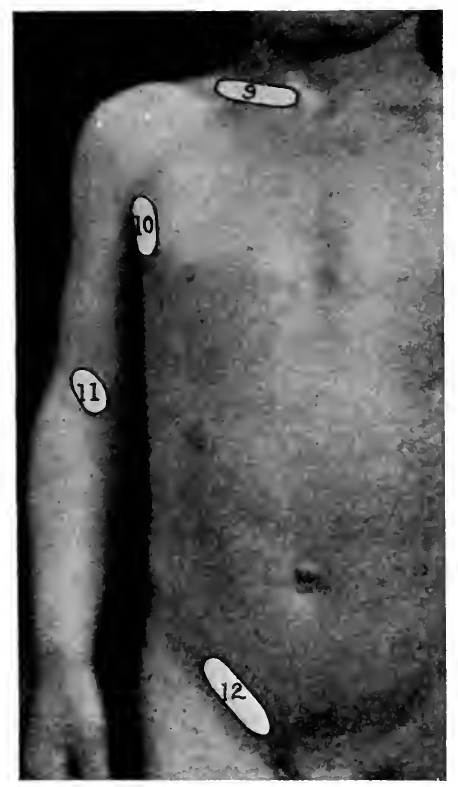

Fig. 69.-Location of lymphatic glands (Kerr).

Lymphatism is the name applied to a general enlargement of all the lymphatic structures in the body. It is due to an extreme susceptibility of the children affected to inflammation of the lymphoid tissues. The tonsils are enlarged, adenoids are present, and the chains of the lymphatic glands are swollen. 
Adenitis is an inflammation of a lymphatic gland.

Varieties.-Simple adenitis, acute or chronic; tubercular; syphilitic.

Adenitis often occurs in the course of the acute infectious diseases, especially in measles and scarlet fever.

Symptoms. - In simple acute adenitis there is a congestion and sweling of the glands. Suppuration is uncommon except after scarlet fever. An infecter wound of the extremities may cause adenitis. Suppuration often takes place under such circumstances.

Tubercular adenitis is common in childhood; suppuration occurs, producing sinuses. The tubercular glands are excised and curetted.

Syphilitic glandular enlargements are occasionally seen in childhood.

Hodgkin's disease is characterized by a general enlargement of the lymphatic glands with progressive anemia, ending fatally. 


\section{CHAPTER XII}

\section{THE INFECTIOUS FEVERS}

\section{CHARACTERISTICS OF FEVER}

Stages.-The febrile stages are invasion, fastigium, and defervescence.

Invasion is the gradual rise until it reaches its maximum.

Fastigium is the stage in which the temperature shows a tendency to reach again and again its highest point, although there may be marked variations.

Defervescence is the gradual fall to normal.

Terminations.-Crisis is a fall of temperature from its height to normal or subnormal within twenty-four hours.

Lysis is a gradual fall to normal taking several days or a week.

Hyperpyrexia is temperature above $106^{\circ} \mathrm{F}$.

Febrile Remissions.-All temperatures of fever show a diurnal remission, usually of one degree. The maximum is at $6 \mathrm{P}$. M. and the minimum at $6 \mathrm{~A}$. $\mathrm{M}$.

Types of Fever.-Contimued Fever.-The diurnal variation is slight, from $\mathrm{I}^{\circ} \mathrm{F}$. to $\mathrm{I} \cdot 5^{\circ} \mathrm{F}$.

Remittent.- The diurnal variation is marked and the minimum temperature remains above the normal.

Intermittent.-The diurnal variation is marked and the minimum temperature is normal or subnormal.

Hectic.-Due to pus. High, irregular fever, with wide fluctuations, accompanied by sweats, chills, and pallor. 
Pulse and Fever Ratio.-There are about ten extra beats of the pulse for every degree of fever.

Simple continued fever without any definite cause is called febricula.

\begin{tabular}{|c|c|c|c|c|c|c|c|c|c|c|c|c|c|c|c|}
\hline$F$. & $E$ & $M$. & $E$. & $M$ & $E$. & $M$ & $E$. & $M$ & $E$. & M. & $E$ & $M$ & E. $M$ & $\angle$ & $M$ \\
\hline 106 & & & & & & & & & & & & & & & \\
\hline 105 & & & & & & & & & & & & & & & \\
\hline 104 & & & & & & & & & & & & & & & \\
\hline 103 & & & & & & & & & & & 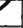 & & & & \\
\hline 102 & & $V$ & & & & & & & . & & & & & & \\
\hline 101 & & & & & & & & & & & & & & & \\
\hline 100 & & & & & & & & & & & & & & & \\
\hline 99 & & & & & & & & & & & & & & & \\
\hline 98 & & & & & & & & & & & & & & & \\
\hline & & & & & & & & & & & & & & & \\
\hline
\end{tabular}

Fic. 70.- Represents a continued fever. It is observed mostly in erysipelas, acute tuberculosis, lobar pneumonia, and typhoid fever (Kerr).

The period of incubation is the period elapsing between the entrance of the poison and the development of the symptoms of the disease. It varies considerably, being

\begin{tabular}{|c|c|c|c|c|c|c|c|c|c|c|c|}
\hline \multirow{2}{*}{$\frac{F}{106}$} & \begin{tabular}{l|l}
$E$. & $N$ \\
\end{tabular} & M. & \begin{tabular}{l|l}
$E$ & $N$ \\
\end{tabular} & a. & $\Lambda$ & $1 E$. & $M$ & $E \cdot M$ & $E . M$. & $E \cdot M$ & \begin{tabular}{|l|l|}
$E . M$. \\
\end{tabular} \\
\hline & & & & & & & & & & & \\
\hline 105 & & & & & & & & & & & \\
\hline 104 & & & & & & $A$ & & & & & \\
\hline 103 & & & $n$ & & & & & & $\Delta$ & & \\
\hline 102 & & & & & & & & 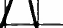 & 1 & & \\
\hline 101 & & & & & & & $V$ & & 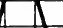 & & \\
\hline 100 & & & $\nabla$ & & $\nabla$ & & & 7 & & & \\
\hline 99 & & & & & & & & & & & \\
\hline 98 & & & & & & & & & & & \\
\hline & & & & & & & & & & & \\
\hline
\end{tabular}

Fig. 71. - Represents the remittent type. This is suggestive of one form of malaria, of tuberculosis (not acute), and suppuration (Kerr).

influenced by the susceptibility of the patient and the virulence of the infection. For the following diseases the average period is:

Typhoid fever-two to three weeks. 
Measles-two weeks.

Rötheln (German measles) - ten to twelve days.

Scarlatina-a few hours to a week.

Smallpox-one to two weeks.

\begin{tabular}{|c|c|c|c|c|c|c|c|c|c|c|c|c|c|c|c|c|}
\hline$F$ & $E$. & $M$. & $E$. & $M$ & $E$. & $M$ & $E$. & $M$ & $F \cdot \Lambda$ & M. & $E$ & M. & $E$. & M. & $E$ & $M$ \\
\hline 106 & & & & & & & & & & & & & & & & \\
\hline 105 & & & & & & & & & & & & & & & & \\
\hline 104 & & & & & & & & & & & & & & & & \\
\hline 103 & & & & & & & & & & & & & & & & \\
\hline 102 & & & & & & & & & & & & & & & & \\
\hline 101 & & & & & & & & & & & & & & & & \\
\hline 100 & & & & & & & & & & & & & & & & \\
\hline 99 & & & & & & & & & & & & & & & & \\
\hline 98 & & & & & & & & & & & & & & & & \\
\hline & & & & & & & & & & & & & & & & \\
\hline
\end{tabular}

Fig. 72.-Represents intermittent fever. The left hand half showing the quotidian type, while the right hand half shows the tertian type. It is significant of malaria (Kerr).

Erysipelas - three to seven days.

Diphtheria - two to ten days.

Varicella-ten to fifteen days.

\begin{tabular}{|c|c|c|c|c|c|c|c|c|c|c|c|c|c|c|c|c|}
\hline$F$ & $E$. & $M$ & $E$ & $M$ & $E$. & $M$ & $E$ & $M$ & $E \cdot$ & $M$ & $E$ & $M$ & $E$ & $M$ & $E$ & $M$ \\
\hline 106 & & & & & & & & & & & & & & & \\
\hline 105 & & & & & & & & & & & & & & & \\
\hline 104 & & & & & & & & & & & & & & & \\
\hline 103 & & & & & & & & & & & & & & & \\
\hline 102 & & & & & & & & & & & & & & & \\
\hline 101 & & & & & & & & & & & & & & & \\
\hline 100 & & & & & & & & & & & & & & & & \\
\hline 99 & & & & & & & & & & & & & & & \\
\hline 98 & & & & & & & & & & & & & & & & \\
\hline
\end{tabular}

FIG. 73.-Represents a hectic and suppurative fever type, which is generally accompanied with sweating (Kerr).

Tetanus-a few days to two weeks.

Mumps-two to three weeks.

The date when rashes appear in various diseases is as follows: 
Typhoid fever-seventh to ninth day. Smallpox - third or fourth day.

Measles-third or fourth day.

Scarlatina-first or second day.

Rötheln-first or second day.

Varicella-first day.

Protection from future attacks conferred by various diseases is as follows:

Typhoid fever-relapses are common; second attack are sometimes seen.

Measles-second attacks rare. What are supposed to be second attacks are usually rötheln.

Rötheln-second attacks rare.

Smallpox-second attacks occasionally occur.

Mumps-second attacks rare.

The following diseases do not confer immunity.

Erysipelas, diphtheria, malaria, influenza, and croupous pneumonia.

An infectious disease means one due to a specific microorganism.

A contagious disease is one which can be communicated by actual contact, either through the person or by infected clothing.

A complication is a condition occurring in the course of a disease.

A sequel appears after the attack.

\section{CEREBROSPINAL FEVER}

This disease is also called spotted fever and epidemic cerebrospinal meningitis. It is a specific infectious disease characterized by inflammation of the cerebrospinal meninges (the membranes covering the brain and spinal cord) 
and usually occurs in the winter and spring. 'The young are more susceptible than the old. The disease is now considered to be contagious. It is caused by a diplococcus.

The mucous membrane lining the nose and pharynx is the portal of entry and dissemination of the disease.

Symptoms.-Common Form.-The disease generally begins abruptly with a chill followed by vomiting and excruciating pain in the head, back, and limbs. The muscles of the head, neck, and back become rigid and contracted so that the head is bent backward and the back is straightened. In severe cases the body may be arched in a state of opisthotonos. The mind is soon

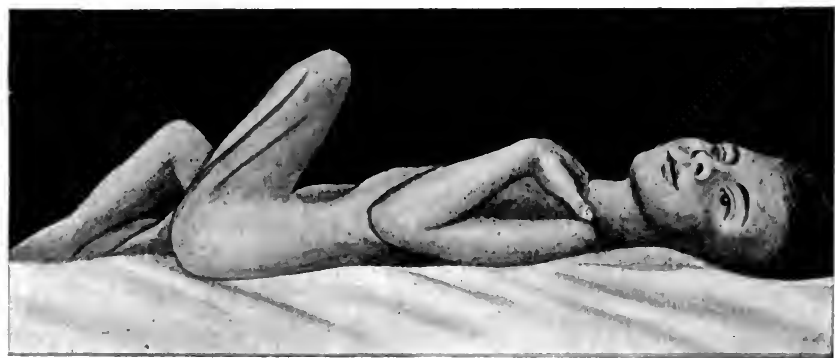

Fı. 74.-Cerebrospinal meningitis: Tåche cérébrale shown on left thigh (Ruhräh).

affected, delirium is rarely absent, and in severe cases it is followed by stupor and coma.

At first there is intense irritation of the whole nervous system, headache is severe and continuous, twitching of the muscles and actual convulsions are common, all the special senses are extremely acute, and there is pain on the slightest movement, which often causes the child to cry out shrilly. Later, when the exudate becomes of sufficient amount to exert pressure, paralysis develops; it may be 
localized, effect one side of the body, or one extremity. Blindness and deafness, disturbed speech, and mental defects are found in the protracted cases. When the finger is drawn over the skin a red line develops; this is called the tâche cérébrale (see Fig. 74).

The temperature is irregular and indefinite in duration; ordinarily it ranges between $\mathrm{IOI}^{\circ}$ to $\mathrm{IO}^{\circ} \mathrm{F}$. In some cases it is almost normal, while in others it is very high. The pulse is rapid and full, the bowels constipated, and there may be polyuria.

The eruption is neither constant nor peculiar. In many cases a blotchy, purpuric rash appears over the entire body. Herpes facialis (fever blisters) are also frequently observed. In other cases urticaria or a roseolar or erythematous rash appears.

The duration ranges from a few hours to sereral weeks.

Treatment.-Within the past two years this dreaded disease has been frequently cured by means of a serum brought to the attention of the world through the work of Flexner and his associates at the Rockefeller Institute in New York. The disease is isolated in most cities under the Board of Health rules.

Nursing.- In nursing the disease follows the same routine described under contagious cases (see page 288). Take every antiseptic precaution and spray the nose and throat frequently with some germicide.

If a lumbar puncture is made by the physician, several sterile test-tubes properly stoppered with sterile cotton should be ready to receive the fluid. (For a description of Lumbar Puncture, see page I92.)

Flexner's serum should be kept on ice before use. At the time it is to be injected the bottle should be placed 
for five minutes in water at a temperature of $100^{\circ} \mathrm{F}$. Do not use boiling water, as it coagulates the serum.

When the bottle is opened its edge and neck should be thoroughly wiped with sterile gauze.

The physician will introduce a hypodermic needle into the spinal canal, as in making a lumbar puncture. He will then fill the syringe, express all the air, and while $d$ drop or two of the fluid is running from the syringe, attach it to the needle. For this reason it is better to have a needle which fits on the syringe, rather than one which screws on. This care is taken to avoid the introduction of air into the spinal canal. He will then inject the fluid very slowly. When the needle is withdrawn, adhesive plaster or a collodion dressing must be at hand to seal the wound.

The child should be prepared for this minor operation in the same way as for a lumbar puncture. The syringe must be absolutely sterile.

Injections are usually given every twenty-four hours.

\section{MALARIAL FEVER}

This is a specific non-contagious disease caused by the hematozoa of Laveran. It is characterized by splenic enlargement, by fever with periodic intermissions or remissions, and by a tendency to extreme anemia.

Etiology.- The exciting cause is the hematozoa of Laveran, and the mode of infection is by the bite of a mosquito which has previously sucked the blood of a malarial patient. All ages, from the newborn to the aged, are subject to malaria.

Manifestations.-Malaria may manifest itself as intermittent fever, remittent.fever, or chronic malarial cachexia. 
Pathology.-The Hematozoa.-A small, colorless ameboid body enters the red blood-corpuscles, increases in size, and becomes pigmented from the coloring-matter of the corpuscles. When the red blood-corpuscle is destroyed the granules of pigment collect in the center of the organism, which finally divides into a number of small hyaline bodies, each of which begins a new cycle of existence. The chills or paroxysms occur at the time these small

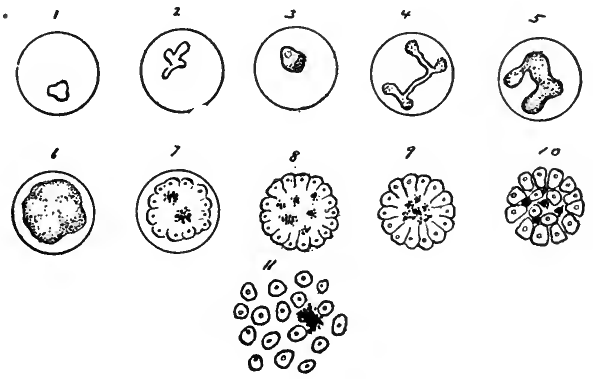

Fic. 75. - Some of the principal forms assumed by the plasmodium of tertian fever in the course of its cycle of development (after Thayer and Hewetson).

bodies are thrown into the blood current after the bloodcells are destroyed, and are due to the production of a poison.

The parasite of tertian intermittent fever requires forty-eight hours to complete its cycle of existence; therefore, when a single group of these parasites exist in the blood a paroxysm occurs every other day. If, however, two groups co-exist and sporulate (the term given to the time when the organisms are thrown into the blood-current) on alternate days, a paroxysm occurs daily (quotidian intermittent fever).

The parasite of the quartan intermittent fever requires 
seventy two hours in which to develop and undergo sporulation; hence, a single group of these organisms in the blood excites a chill on every fourth day. When two groups co-exist a chill occurs on two successive days and is followed by daily intermission. When three groups co-exist a chill occurs every day and there is quotidian intermittent fever again. The life-history of the parasite of remittent fever within the body is not definitely known. Its cycle of existence occupies from twenty-four to fortyeight hours.

In advanced malaria the blood shows a diminished number of red blood-corpuscles and a large quantity of

\begin{tabular}{|c|c|c|c|c|c|c|c|c|c|c|c|c|c|}
\hline Temp. & ${ }^{\prime}{ }^{\prime} E$ & $\begin{array}{c}2 \\
M E\end{array}$ & $\begin{array}{c}3 \\
\text { M.E }\end{array}$ & $\begin{array}{c}4 \\
M . E\end{array}$ & $\begin{array}{c}5 \\
M . E\end{array}$ & $\begin{array}{c}6 \\
M . E .\end{array}$ & $\begin{array}{c}7 \\
M \cdot E\end{array}$ & $\begin{array}{c}8 \\
M . E\end{array}$ & $\begin{array}{c}9 \\
\text { M.E. }\end{array}$ & $\begin{array}{c}10 \\
M . E\end{array}$ & $\begin{array}{l}\prime \prime \\
M . E\end{array}$ & $\begin{array}{l}12 \\
\text { M.E. }\end{array}$ & \\
\hline 108 & & & & & & & & & & & & & \\
\hline 107 & & & & & & & & & & & & & \\
\hline 106 & & & & & & & & & & & & & \\
\hline 105 & & & & & & & & & & & & & \\
\hline 104 & & & 1 & & & & & & & & & & \\
\hline 103 & & & & & & & & & & & & & \\
\hline 102 & & & & & & & & & & & & & \\
\hline 101 & & & & & & & & & & & & & \\
\hline 100 & & & & & & & & & & & & & \\
\hline 99 & & & & & & & & & & & & & \\
\hline 98 & & & & & & & & 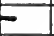 & & & & & \\
\hline 97 & & & & & & & & & & & & & \\
\hline
\end{tabular}

FIG. 76. - Tertian type of malarial fever. Male child of six years. Quinin begun at $\boldsymbol{X}$ (Kerr).

free pigment. The spleen is greatly swollen and deeply pigmented. All the organs, including the brain, are discolored by this free pigment.

Intermittent Malarial Fever.-Symptoms.-The characteristic features of this form of malaria are the intermittent type of fever, the enlargement of the spleen, the hematozoa in the blool, and the occurrence at regular 
intervals of the paroxysms divided into three stagesthe chill, the fever, and the sweat.

Cold Stage.-There is malaise, headache, and great chilliness. The features are pinched, the lips are blue, and the surface of the body is cold and covered with "goose flesh." The rectal temperature is high-104 ${ }^{\circ} \mathrm{F}$. to $105^{\circ} \mathrm{F}$. Vomiting may occur. The chill lasts from a few minutes to an hour or two.

Hot Stage.-The surface temperature gradually rises, the skin becomes hot, the face flushed, the eyes injected, and the pulse rapid and full. The temperature in the axilla may reach $106^{\circ} \mathrm{F}$. to $107^{\circ} \mathrm{F}$. The child complains of severe pain in the head, back, and limbs, and of thirst. The urine is scanty and dark colored. This stage usually lasts from one to five hours.

Sweating Stage.-The fever gradually subsides, the pain grows less, free perspiration follows, and the child falls asleep from which he awakens feeling fairly well.

Varieties.-When the disease occurs every day it is termed quotidian intermittent fever; every other day, tertian intermittent; every fourth day, quartan intermittent fever.

Prognosis.-Always favorable. Even when no treatment is instituted the paroxysms gradually subside. Chronic malarial cachexia may develop.

\section{Remittent Malarial Fever or Estivo-autumnal} Fever.-In temperate zones remittent fever is observed chiefly in the autumn. It is uncommon in children who live outside of malarial districts.

Symptoms.-There is malaise with moderate chilliness followed by a continuous fever which daily remits. The maximum temperature ranges from $103^{\circ} \mathrm{F}$. to $106^{\circ} \mathrm{F}$, 
and while this lasts the skin is hot, the face flushed, the eyes injected, the pulse full and strong, the urine scanty, and the patient complains of pain in the head, back, and limbs. Definite paroxysms may or may not be present. Delirium is sometimes noted, vomiting often occurs, and jaundice may appear from the destruction of the red bloodcorpuscles and the liberation of their pigment. The spleen is enlarged and the hematozoa are found in the blood.

Prognosis.-Favorable. The average duration is from one to three weeks.

Chronic malarial cachexia is characterized by anemia, a sallow appearance to the skin, and splenic enlargement.

Etiology.-It may result from repeated acute attacks of the disease or it may develop as a primary condition from slow infection.

Symptoms. - The child is thin and pale, the complexion is of a dirty yellow or muddy hue, fever is often absent or if present, it is slight and irregular. The spleen is considerably enlarged. There is great weakness from the attending anemia. Headache and neuralgia are common symptoms. Hematuria is sometimes observed.

Prognosis.-Guarded. With the spleen very large and extreme anemia the patients rarely recover. Malarial infection seems to predispose to certain cases of dysentery, pneumonia, and amyloid degeneration of the viscera.

Treatment.-As malarial fever is contracted by means of infection through mosquito bites, proper measures should be taken to protect children from the mosquitoes. Just as great care should be taken to prevent mosquitoes from biting children who have malaria, and in this way preventing the spread of the disease. Quinin is a specific rem- 
edy, killing the hematozoa. The dose is from 5 to ro gr. a day in divided doses (four years), and in ordinary cases in older children is from 15 to 20 gr. a day in divided doses. The drug is given so that the last dose is taken two hours before the expected chill. The cold stage is treated with hot-water bottles and blankets, and the hot stage by sponging.

\section{SYPHILIS}

Syphilis is a communicable disease and may be acquired or hereditary. It is caused by the spirochæta pallida.

Syphilis is acquired usually from the mother of the child or from syphilitic wet-nurses. It follows the same course as syphilis in the adult and is divided into three stages. The first is characterized by a chancre, the second by a rash, and the third by a bone lesion and ulcerations. It is now considered contagious in all stages, but especially in the second stage.

Hereditary syphilis is more common. When born the child at times has large blebs on the skin surfaces and scars develop around the lips called rhagades.

Symptoms.-The Bones.-Epiphysitis, an inflammation of the ends of the bones, is present. Later in the disease chronic osteoperiostitis and syphilitic dactylitis are seen.

The liver and the spleen are enlarged.

The Respiratory Tract.-Pneumonia is common. Ulcers of the larynx are sometimes observed.

Digestive Tract.-A chronic catarrh of the pharynx is present, causing "snuffles."

The Organs of Special Senses.-Otitis media and interstitial keratitis are common. 
Nervous Symptoms.-Often absent, but there may be impairment of mentality.

The children are weak and sickly and usually die young.

If three months pass after a child is born from syphilitic parents without the appearance of any characteristic symptoms, the child will, in all probability, escape.

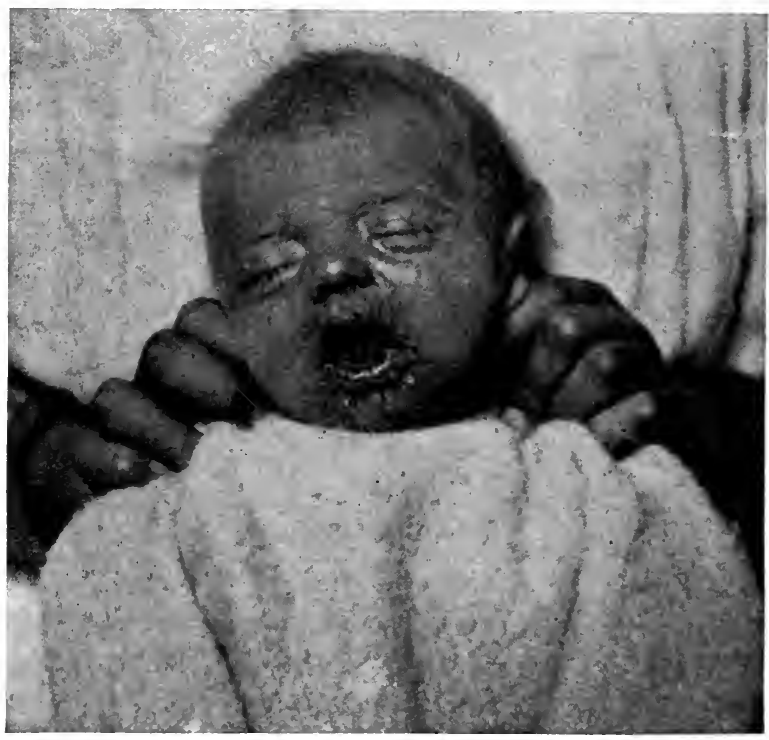

Fig. 77.- Hereditary syphilis: radiating fissures of the lirs (after A. Fruhinsholz).

Hutchinson's Tecth.-If a child suffering from hereditary syphilis lives, the second or permanent teeth are characteristic (sce Fig. 4).

The teeth most frequently affected are the upper central incisors. They have a dull, opaque color and have a roughly rounded and stunted appearance. The 
cutting edge of the tooth is narrower than its neck. Over the tips of these stunted and conic teeth the enamel is irregular and forms a semilunar notch.

Treatment.- The treatment of both hereditary and acquired syphilis consists in giving mercury. This, in infants, is given in the form of ointments. Great care must be taken by the nurse to avoid contamination in handling syphilis.

A new method of arsenical treatment has been advanced by Ehrlich which seems to give excellent results. It is, however, too early to tell the actual value of this remedy in childhood.

\section{TETANUS OR LOCKJAW}

An acute infectious disease excited by the tetanus bacillus and characterized by painful tonic spasms of the voluntary muscles.

The bacillus gains an entrance to the system through some wound. Lacerated and punctured wounds, burns, and frost-bites are most likely to become infected. Stables seem to be the breeding ground for the bacillus.

Symptoms. - The disease begins with rigidity in the muscles of the neck and lower jaw, by degrees the muscles of the back, abdomen, and extremities are similarly involved. The face has a peculiar expression, the brow is wrinkled, the corners of the mouth are drawn up (the sardonic grin) and the jaws are tightly closed (trismus). The body may become arched in a position of opisthotonos. There is extreme hyperesthesia, and the slightest touch causes an increase in the spasm which is attended by severe pain. If the respiratory muscles are involved there is intense dyspnea. The temperature usually re- 
mains normal until just before death, when it may rise to $107^{\circ} \mathrm{F}$. or more. The mind is clear to the end.

The duration is from a few days to several weeks. Death occurs in nearly every case.

Tetanus occurs sometimes in the newly born from infection of the umbilical cord.

Treatment.-There is an antitoxin for tetanus which is injected into the system.

\section{HYDROPHOBIA OR RABIES}

A disease of dogs which at times is communicated to children through a bite of an animal suffering from rabies. It takes about six weeks for the disease to develop after the wound is received.

Symptoms.-These consist principally of paralysis of the muscles of the throat, which prevents swallowing. There is fever, and convulsions are present.

Treatment.-All dog bites should be immediately washed with antiseptic solutions and a wet bichlorid dressing applied. At the present time cauterization has been discontinued, the bite being treated as an open wound. If the dog is known to have had rabies, treatment by the Pasteur method will prevent the child from developing the disease. Pasteur institutes exist in New York and Chicago. The Boards of Health of some of the larger cities furnish the serum. The treatment, according to this method, consists of hypodermic injections of the serum extending over a period of several weeks.

\section{NURSING IN THE ACUTE INFECTIOUS DISEASES}

The room should be kept at an even temperature; it should be well lighted and ventilated. Bathing should be thorough. 
The clothing should be of a light woolen texture. Sleep should be encouraged, the urine should be examined routinely, and the temperature, pulse, and respirations taken every three hours where there is fever.

The nurse should roll up her sleeves when nursing an infectious disease.

Care should be taken by the nurse to prevent the infection of herself. All antiseptic precautions should be employed. In malaria the child should be protected against mosquitoes.

She should avoid all possibility of being scratched or bitten in hydrophobia. This may occur if it is necessary to feed by gavage.

The feedings should be liquid. In tetanus it may be necessary to feed through a catheter placed in the mouth or nose; it may be necessary to chlorofrom the child while doing this.

In syphilis avoid contagion, and when applying mercurial ointment, to prevent irritation of skin, select a new place each day. For example, the abdomen, right and left axillæ, and the right and left thighs can be utilized in rotation.

A glass rod is the best means of applying mercurial ointment; I dram is used, and is rubbed in for a half hour. 


\section{CHAPTER XIII}

\section{TYPHOID FEVER}

TyPHOID FEver is an acute infectious fever due to the typhoid bacillus or bacillus of Eberth.

The disease is especially characterized by pathologic changes in the lymph-follicles of the intestines and particularly of Peyer's patches, by changes in the mesenteric glands, and by an enlargement of the spleen. The lesions in the intestines and the mesenteric glands are ulcerative in character.

Typhoid fever is recognizable in the writings of the ancients four hundred years before Christ, and ever since that period epidemics of this disease have been constantly recorded in medical history. The causes for the frequent epidemics are better understood when it is known that the typhoid bacillus shows great resisting powers. They have remained alive for three months in distilled water, and when buried in the upper layer of the soil they will retain their vitality for six months.

Dissemination of the Disease.-The method by which the typhoid bacillus spreads the disease is well understood. The stools of a person suffering from the disease are filled with the germs. Unless these stools are covered with some solution like carbolic acid, $\frac{1}{20}$; bichlorid of mercury, $\frac{1}{2000}$; or chlorinate of lime, of equal strength, they pass with the sewage to the nearest water-course. Once in the stream they may pollute an oyster bed or they 
may be contained in the water used to sprinkle green vegetables. By far the greatest danger is in swallowing the bacilli in drinking water. Milk may be contaminated by water containing the bacillus.

The distances they travel, at times, is almost incredible. Miles down a stream an epidemic will arise traceable to one case at its source. A case occurring in the fall of the year can produce typhoid after the spring thaws, if the stools are carelessly deposited on the upper surface of the soil.

The relatively infrequent communication of typhoid fever to physicians and nurses is explained by the fact that the contagion escapes from the child in the stools alone, and as these are promptly disposed of, the chances of dissemination of the poison are few. Carelessness in the disposition of these discharges, such as permitting them to dry upon the linen and in this manner allowing the bacillus to pass into the air of the room, at times occasions the infection of the nurse, the physician, and others attending a typhoid case.

Age.-Typhoid fever is seen most often during adolescence and in adult life before thirty years of age, although it occurs frequently in childhood. It is rare before two years of age. In the young the duration of the disease is short, and the prognosis very favorable.

Sex.-Typhoid fever seems to be more prevalent among boys than among girls, probably because of their more frequent exposure rather than greater susceptibility.

Season.-Typhoid fever is more common in the late summer and in the early autumn months than at any other time of the year; hence one of the names for typhoid is autumnal fever. 
Morbid Anatomy.-The principal lesions in typhoid fever are in Peyer's patches in the ileum (the lowermost portion of the small intestines). Peyer's patches are a collection of lymphatic glands in the walls of the intestines and are from one to three inches in length. The typhoid bacilli implant themselves in these glands and cause an inflammation. The Peyer's patch becomes swollen and the superficial tissue sloughs off, leaving a raw, ulcerating surface. This is called a typhoid ulcer. These ulcers may be very small, from an eighth to a quarter of an inch in diameter. More often there is a large, elliptic ulcer, a whole Peyer's patch being involved. At times by the union of one or more ulcers much larger ones are formed, especially at the lower end of the bowel. The borders of the ulcers are raised, the floor of the ulcer is usually the submucous, or muscular coat of the bowel. (The bowel has four coats, the mucous, the submucous, the muscular, and the peritoneal.) If the ulcer eats through all these coats there is a perforation of the bowel. The discharge of the contents of the intestines through the perforation into the peritoneal cavity is often followed by a fatal peritonitis. More commonly the ulcer heals before the bowel is perforated, and the patient recovers, but the normal glandular substance is never restored at the seat of the ulcer.

At autopsies ulcers are discovered at different stages of healing, sometimes they are all healed with the exception of the single fatal spot, which has become the seat of perforation. The large intestines are also invaded at times; perhaps in about one-third of the cases perforation may take place here and also in the appendix where the process occasionally extends. Similar infiltration of the 
lymph nodules and the lymph cords of the mesenteric glands and of the spleen occurs, contributing to the enlargement of these organs. In the spleen it is associated with active congestion which causes a further enlargement, generally recognizable during life. There has even been known to be a rupture of this organ. Changes in the liver, kidneys, and in the respiratory organs are often found. Hypostatic congestion of the lungs is a frequent complication. Thrombosis of the veins, especially of the femoral, causes the not very rare symptom of milk-leg. Endocarditis is sometimes found. Notwithstanding the intensity of the nervous symptoms in some cases, meningitis is rarely met with. Abscess of the parotid gland is a familiar complication.

Characteristics of Typhoid Fever in Children.During childhood the disease is of shorter duration, milder course, has fewer complications, and has a lower mortality than in adult life.

The onset is more sudden, fever, vomiting, and prostration being seen as often as slow insidious beginnings. Constipation is more frequent than diarrhea, tympanites is not marked, and the eruption is less constant in childhood. Nervous symptoms are not as frequently found as in adults; hemorrhage and perforation are also met less often. Noma is sometimes a complication. Death rarely occurs in uncomplicated cases. After ten years of age the symptoms are similar to those seen in adult life.

Symptoms and Course.-A certain period of incubation is necessary after the successful implantation of the bacilli before typhoid fever arises. This varies from a few days to two weeks and even longer in some cases. The period of incubation may be without symptoms, but a sense of 
weariness and indisposition to play are usually present. The latter often can be overcome by force of will. A want of appetite and a slight coating of the tongue are not infrequent. In older children the disease itself usually sets in gradually and often is quite advanced before it is suspected; indeed, at times well advanced, constituting the so-called walking typhoid. In children under ten years of age the onset is less gradual.

Symptoms of the Attack.-There may be headache, anorexia, a furred tongue, nausea, chilliness, but seldom a decided rigor. The disease may be ushered in by pain in the back or leg muscles and there may be nosebleed (epistaxis). In older children there may be a looseness of the bowels. There is continuously a slight fever and the child feels wretched. The fever and discomfort increase and finally the child goes to bed. The tendency to looseness of the bowels and epistaxis justify a strong suspicion of the existence of typhoid fever. Yet one or both are frequently absent, and in younger children constipation is more often seen than diarrhea. The abdomen soon becomes slightly distended and pressure on the right iliac fossa elicits tenderness with gurgling.

Rash.-Usually about the eighth day, rarely later and sometimes a little earlier, the rose-colored spots make their appearance on the skin of the abdomen and chest and at times elsewhere on the body. They are mostly bright red in color, disappear on pressure, and reappear instantly. They are very slightly, if at all, raised. Their number varies greatly. Sometimes they are very numerous, but more often but five to ten are discovered.

Herpes are very rarely seen, in contradistinction to pneumonia. 
The fever is at once the most important and the most characteristic symptom, and from the temperature alone a diagnosis can be suspected.

Initial Stage.-During the first week of the fever there will be found a peculiar tide-like evening rise and morning fall, the temperature of each morning and evening being from one and a half to three degrees higher than that of the morning and evening previous.

The fastigium is the stage when the fever again and again reaches the highest point. At the end of a week the height of the fever is reached and then it continues with but little variation, the evening rise and the morning fall still being characteristic, but the remission being less than that seen at the onset-from a half to two degrees being found. It is during this period that the maximum temperatures are found; often $105^{\circ} \mathrm{F}$. or a little above are noted. A temperature of $106^{\circ} \mathrm{F}$. is frequently followed by recovery, and while temperatures of $107^{\circ} \mathrm{F}$., $108^{\circ} \mathrm{F}$, and even $109^{\circ} \mathrm{F}$. are sometimes seen, they usually result fatally.

The fastigium is succeeded by a third stage or stage of decline in which the reverse of the initial stage is shown by an evening temperature lower than that of the previous evening and the morning temperature lower than that of the previous morning, but the evening temperature still higher than the morning temperature of the same day. This decline continues until the normal is reached, and at times from one to two weeks are consumed before this is attained. The duration of the fever in children, however, is usually shorter than in adult life.

Sudden falls of a decided character may occur in consequence of hemorrhage from the bowels or even from the 
nose, or from collapse after perforation of the bowels. Sudden rises are produced by indiscretions in diet or by the supervention of some acute inflammatory condition, such as pneumonia. The skin is usually dry, although profuse sweating sometimes occurs most frequently after a bath. Children frequently have higher temperatures than adults.

The pulse is not very frequent, 90 to 120 is the usual average. In grave cases it maintains a frequency of I 40.

\begin{tabular}{|c|c|c|c|c|c|c|c|c|c|c|c|c|c|c|c|c|}
\hline & & 1 & 2 & 3 & 4 & 5 & 6 & 7 & 8 & 9 & 10 & 11 & 12 & 13 & 14 & 15 \\
\hline$P$ & $T$. & m.e. & mi.e & m. $e$ & m.e. & m.e & m.e & m.e. & m.e. & in.e & m.e & in.c. & $m . c$ & $m . c$ & m.e & $m \cdot e$ \\
\hline 170 & 108 & & & & & & & & & & & & & & & \\
\hline 160 & 107 & & & & & & & & & & & & & & & \\
\hline 150 & 106 & & & & & & & & & & & & & & & \\
\hline 140 & 105 & & & & & & & & & & & & & & & \\
\hline 130 & 104 & & & & & & & 1 & $i$ & & & & 八 & & & \\
\hline 120 & 103 & & & & & & & & & & & & & & & \\
\hline 110 & 102 & & & & $v^{\prime}$ & & & & & & & $i$ & & $y^{i}$ & & \\
\hline 100 & 101 & & & & & & & & V & 4 & $x$ & & & & & \\
\hline 90 & 100 & & $\because$ & & & $\alpha$ & & & & & & & & & & \\
\hline 80 & 99 & & & & & & & & & & & & & & & \\
\hline 70 & 98 & & & & & & & & & & & & & & & \\
\hline 60 & 97 & & & & & & & & & & & & & & & \\
\hline
\end{tabular}

FIG. 78.-Chart of the temperature (-) and pulse (--.) in typhoid fever of moderate severity in a male child five years old (Kerr).

The pulse is often dicrotic (see page 16I). The breathing rate commonly advances with the rate of the pulse. The heart-sounds at first are normal, but gradually grow less distinct as the prostration increases.

As the disease advances the tongue, previously furred, tends to become dry and brown, clearing at the edges, however, and also at the tip as the case improves. In severe cases, especially, if the mouth is not kept clean; sordes form on the teeth, stomatitis sets in, and the lips are covered with black crusts. 
Diarrhea, if present, is rarely troublesome. The stools are apt to be grayish-yellow and of about the consistency and frequently likened to pea soup.

Hemorrhage from the bowel is always a serious complication, but by no means fatal, though large quantities of blood are sometimes discharged. Very rarely a patient will bleed to death. Following the hemorrhage there will be a marked fall in the temperature and a pallor and a faintness such as is common to large hemorrhages elsewhere.

Perforation is suspected when there is a sudden pain in the side with spreading tenderness and vomiting. The temperature falls and the pulse becomes more rapid. If these symptoms occur during the course of typhoid the physician should be immediately informed.

Tympanites or distention of the abdomen in a mild degree is at times present in children, but not so often as in adults. The accumulation of gas is commonly ascribed to atony of the bowel.

Delirium is rare in childhood.

A tendency to drowsiness or even stupor is present, and from this the name of the disease is suggested. Muscular tremor is a symptom in severe cases. Carphalogia or picking at imaginary objects is sometimes present and is merely a symptom of the typhoid state; it is not necessarily of fatal import, as is so often thought by the laity. When this condition, known as the typhoid state, sets in the tongue becomes dry, brown, and fissured.

Slight cough usually sets in as the disease advances, due to a hypostatic congestion of the lungs. This can be relieved by frequently changing the position of the child in bed. 
The spleen is always enlarged, reaching in the first half of the second week two or three times its normal size. It then gradually diminishes. Tenderness may accompany the enlargement.

The urine in typhoid cases is always dark hued and concentrated, with correspondingly high specific gravity. Often when the fever is high there may be a slight albuminuria. If there is a nephritis present there will be casts in addition to the albumin.

The blood in typhoid fever shows a slight diminution in the white blood-corpuscles in contradistinction to pneumonia where they are greatly increased. There is also a reduction in the red blood-corpuscles and the hemoglobin, according to the severity of the case.

The Widal reaction depends upon the ability of the blood from a typhoid patient to agglutinate a pure culture of typhoid bacilli. It is positive in about 95 per cent. of all cases of typhoid, so it is practically a sure sign of the disease.

The diazo reaction is a test made with the urine.

Sequelæ.-Insanity in the form of acute mania sometimes occurs. Aphasia and chorea have been reported. Phthisis, post-typhoid bone lesions and typhoid spine (a severe pain in the back aggravated by motion) follow the disease. As a rule sequela are uncommon in children.

Relapses often occur following upon the relaxation of diet. The symptoms of a relapse are those of the primary disease, excepting that the symptoms are less severe, the duration shorter, and recovery the rule. Relapses may be multiple.

Recrudescence is a simple return of fever, also often induced by lapses in diet. 
Prognosis.-In children under fifteen recovery almost invariably takes place. Hemorrhage and perforation occur less often than in the adult.

A mistake is often made in using the term typhoid pneumonia, meaning typhoid fever complicated by pneumonia. Typhoid pneumonia means pneumonia with typhoid symptoms, such as the brown, dry tongue, delirium, twitching of the tendons, and picking at the bedclothes. These occur in fevers when patients are exhausted, and are not seen in typhoid fever alone. In typhoid pneumonia, typhoid fever does not exist. If the two are present at the same time use the title typhoid and pneumonia.

Treatment and Nursing.-Absolute rest and restriction of diet are essential. Many physicians use a liquid diet, others a more liberal one. It is usually governed by the amount of emaciation and tympanites.

The treatment of typhoid fever by graduated baths is far better than by any other method. It not only reduces the temperature, but controls the delirium, the diarrhea, and keeps the mouth and tongue in good condition. It also lessens the severity of every symptom. The method of giving these baths is described on page 396). The effect of the bath on the temperature varies with the stage of the disease, it being frequently the case in the first week that the fall in temperature is less than one degree; in the second and third week, however, the fall will be from one to three degrees. In addition to lowering the temperature the immediate effect of the baths is to add strength to the heart and volume to the pulse (Holt).

Contra-indications. - Practically the only contra-indications to this plan of treatment are: (I) When there is an 
almost absolute pulselessness with a blue, cyanosed appearance of the skin occurring while giving the tub, in which case the child should be immediately removed, put to bed, and hot-water bags applied to the extremities and whiskey given. (2) Hemorrhage. (3) Perforation. None of the complications excepting hemorrhage from the bowel and perforation are allowed to interfere with the carrying out of this treatment. The reason they are discontinued in hemorrhage is the fear that the necessary movements in the bath would excite further bleeding; and in perforation, to avoid the danger of unnecessary contamination of the peritoneum with feces. Delirium is controlled by a soothing voice and touch in conjunction with the bromids.

Constipation should be relieved by the enemas alone.

Hemorrhage from the bowel should be treated by absolute rest, cold to the abdomen, the food should be reduced to a minimum, and be of the mildest character. In severe cases the foot of the bed can be raised. The physician should be immediately notified.

Perforation.--Symptoms of perforation, such as spreading tenderness, sudden pain in the side and vomiting, demand absolute quiet and the immediate notification of the physician.

Tympanitic distention may be relieved by the careful passage of the rectal tube, and the pain relieved by turpentine stupes.

One of the dangers of protracted cases is bed-sores. These can generally be averted by careful attention to cleanliness, by thoroughly drying the patient after washing, by removing all traces of urine or other discharges, and by sponging the patient daily with alcohol or whisky. 
Above all, the position in bed should be changed frequently and all inequalities in the bed-clothes should be kept smoothed out, while the bed should be kept clear of crumbs and other particles of food. Should a sore appear, antiseptic dressings should be applied and the part relieved from pressure by an air-cushion.

Convalescence.-In no disease is watchfulness during convalescence more important. Relapses and the perforation of an ulcer may occur during this period. The ulcers are not necessarily healed when the temperature reaches the normal. Therefore, absolute rest and a liquid diet should be kept up for at least ten days after the temperature has reached normal. The hair is very apt to fall out and, therefore, should be cut short. By no means is it necessary to shave the head.

Prophylaxis. - When typhoid is epidemic all drinkingwater should be boiled for one-half hour before using. Since the contagion of typhoid fever resides solely in the stools, vomited matter, urine, and other discharges from the body, it is important that these should be thoroughly and properly disinfected, also that the linen which has been in the least degree soiled by them should be immediately removed and sterilized.

Stools may be disinfected in the following manner: Place in the bed-pan, before using, a small quantity of carbolic acid solution, I : 20 , or chlorinated lime of the same strength. Cover the evacuation with another quantity of the disinfectant, mix thoroughly, stand aside for one-half hour, then empty the whole into the water-closet hopper. Then thoroughly rinse out the vessel with disinfectant solution and hot water. Linen may be immersed in carbolic acid, I : 20, until boiled. Boiling for half an hour disinfects it. 
After the bowels have been moved the buttocks and anus of the patient should be thoroughly washed with $\mathrm{I}$ : 40 carbolic acid or bichlorid, I to 2000 , followed by hot water and soap.

Door-knobs or parts of the door touched with the soiled hands should be washed with the same disinfectants, since drying of the fecal matter causes it to disseminate and gives rise to a source of infection.

The nurse should wash her own hands with soap and water and then rinse them in a solution of bichlorid. It is by soiled hands or floating bacteria from dried feces that nurses are infected.

Where tub-bathing is employed, it is possible that the water of the bath that has been used several times may be a source of infection. The nurse should, therefore, wash her hands after tubbing a patient, and watchful care should be exercised not to carry them to the mouth during the bath.

After death or discharge of the patient the mattress, which should be well protected during use by a rubber sheet, should be thoroughly aired. The rubber sheet itself should be washed in carbolic acid, rinsed in cold water, and dried in the open air. 


\section{CHAPTER XIV}

\section{TUBERCULOSIS}

Tuberculosis is an infectious, communicable disease due to the bacillus tuberculosis of Koch. It is a disease that may occur at any time of life, most frequently in childhood and between the ages of twenty and thirty. It may be local or general and may involve any organ and almost any structure of the body.

The predisposing causes to tuberculosis are divided into general and local.

General predisposition to the disease may be inherited directly from the parents, who have themselves suffered from tuberculosis, or from those who, in consequence of syphilis, alcoholism, or any other constitutional vice, have transmitted a feeble constitution to their children. Actual congenital tuberculosis is rare; that is, a child is rarely born with tuberculosis, although cases are sometimes seen. The surroundings in which a child is reared plays an important part in the general predisposition to tuberculosis, cramped quarters and exposure being the chief causes. In childhood marasmus, intestinal diseases, in fact any debilitating general or local disease, may predispose to tuberculosis.

A local predisposition is created by any diseased condition of the mucous membranes or organs most exposed to the infection. The most important are repeated attacks of bronchitis, bronchopneumonia or pleurisy, and chronic 
catarrhal inflammations of the nose and pharynx so frequently associated with enlarged tonsils or adenoid growths of the pharynx.

The rôle played by other diseases in the development of tuberculosis is important and, until recently, little understood. In a very large number of cases tuberculosis develops as a sequel of one of the acute infectious diseases, particularly measles, pertussis (whooping-cough), or epidemic influenza. In such cases there probably has existed a previously latent tuberculosis, usually an involvement of the bronchial lymph-nodes. An acute disease, like pertussis, lowers the general vitality of the body and gives the tubercular process a chance to light up. As long as the constitution of the child is robust and the resisting powers of the tissues are up to par there is slight danger of contracting any form of tuberculosis. It is when the resisting powers of the tissues are faulty that the bacilli gain a foothold, and this may be in the mucous membrane of the throat, lungs, or bowel. The bacilli are taken up by the lymphatics and carried to the nearest lymph-gland, where they are arrested. The glands involved may be the cervical lymph-glands or the bronchial lymph-glands which are back of the bifurcation of the bronchi and receive the lymphatics from the lungs; or the mesenteric lymph-glands which are retroperitoneal (behind the abdominal viscera) may be involved. When the bacillus enters one of these glands it starts an inflammation which, if allowed to run its course, will be the typical inflammation produced by the tubercle bacillus; namely-congestion, swelling, cell proliferation, and caseation. Or if the vitality of the body is regained this process may be arrested at any point and the product of the inflammation will 
become encapsulated by a wall of fibrous tissue (the way a tubercular lesion is cured), in which condition they may lie latent in the body for an indefinite number of years and possibly for a life-time. Tubercular cervical adenitis is frequently seen in the hospital wards; tubercular lymphglands, both bronchial and mesenteric, are met with as often in the autopsy-room.

If for any reason the vitality of the part is not strong enough to resist the tubercular inflammation, or if some intercurrent disease lowers the vitality sufficiently to permit a lighting-up of the old tubercular inflammation, sooner or later these lymph-glands will caseate and rupture. In the cervical glands such a condition leads to the sinuses of the neck so frequently seen, while in the bronchial glands such a condition leads to a tubercular infection of the lungs known as phthisis. In the mesenteric glands the rupture leads to absccss formation, peritonitis, or tubercular enteritis.

Any structure of the body may be involved by the tubercle bacillus. When the spine is affected there is Pott's disease, when the bones and joints are affected there is tubercular osteomyelitis, coxalgia, and other surgical conditions. The liver, spleen, pleura, kidneys, and all other organs may be involved, usually secondarily.

The bacillus does not always take the above course. It may enter the lungs directly by inhalation and set up a tubercular lesion, or it may be swallowed in the milk and meat from tubercular cattle.

In young children under two years of age the lung is the part of the body usually affected; beginning with the second year tubercular meningitis is more frequently. found; and after the third year tuberculosis of the bones, 
of the cervical and mesenteric lymph-glands, and of the peritoneum and intestines become more frequent and are seen throughout childhood.

That the disease is communicable is proved by large numbers of individual cases in which a person closely associated with the tubercular patient has contracted the disease and died. It is sometimes hard to trace the origin of a given case of tuberculosis on account of its tendency to lie latent for so long a period.

In addition to the above predisposing causes residence in a low, damp, and badly drained locality, and physique contribute to the tendency toward developing tuberculosis.

The physique of the child is important. Children who suffer with tuberculosis of the lungs usually have a flat chest with prominent shoulder-blades and a narrow angle of the ribs at the lower border of the anterior portion of the chest. This is called the phthisic chest. It seems to predispose to tuberculosis of the lungs on account of the poor expansion of the lungs which results from such a formation. Many children have phthisic chests who are not suffering from the disease. Phthisis is the name applied to tuberculosis of the lungs.

The varieties of tuberculosis seen in children are tuberculous bronchopneumonia, miliary tuberculosis, tuberculosis of the bronchial lymph-glands, tubercular meningitis, and tubercular bone and joint diseases.

\section{TUBERCULOUS BRONCHOPNEUMONIA}

Pathology.-If the tubercle bacillus does not gain access to the lungs from the rupture of the bronchial lymph-node the bacillus entering the respiratory tract through the inspired air lodges in the terminal bronchioles 
and excites a proliferation or overgrowth of the fixed cells. The new cells are termed epithelioid and frequently contain bacilli. Giant-cells are often formed by a fusion or overgrowth of these cells. This aggregation of new cells acts as an irritant and is soon surrounded by a wall of leukocytes, the whole forming a gray, translucent mass, the so-called gray tubercle. In a short time the bacillus excites a necrosis or softening which starts in the center, spreads to the periphery and converts the tubercle into a yellow, cheesy mass called the yellow tubercle. The degenerated tubercles fuse and form the uniform cheesy masses commonly observed at the autopsies. At this stage one or two things may occur. Either the mass may soften, break into a bronchial tube, and leave behind a cavity with ulcerating walls, or it may become encapsulated by an overgrowth of connective tissue and later calcify. In addition to the tubercular process other changes are noted. The lung tissue in the neighborhood of the tuberculous deposits is the seat of a true bronchopneumonic inflammation, the connective tissue is always more or less proliferated, the bronchial tubes are inflamed, and the pleura over the affected area is always adherent.

In infants the disease resembles marasmus, and it is very hard to distinguish the two conditions.

In older children the disease follows one of three courses: namely, a rapid, acute course, a subacute course, and a chronic course.

Symptoms.--In the acute form the disease resembles bronchopneumonia. The characteristic symptoms of phthisis are absent. The temperature remains high, without intermission, and there is a gradual loss of flesh and strength. Death results in every case. 
In the subacule variety the principal symptoms are fever and wasting. The temperature is irregular. Cough and dyspnea are present, expectoration is absent before four years of age. Hemoptysis is rare, respirations are accelerated, the spleen is often enlarged, anemia is marked, dropsy is rare, and then only late in the disease. Sweating is also seen late in the disease.

The chronic cases resemble protracted bronchopneumonia. Often periods of freedom from the disease are observed. At the first fresh cold the symptoms reappear. This condition of affairs may continue over an indefinite period. It may terminate by becoming acute or subacute in form, when the symptoms observed in those conditions will be found. It may cause general miliary tuberculosis or tubercular meningitis. The presence of the tubercle bacillus in the sputum makes the existence of the disease positive.

\section{ACUTE MILIARY TUBERCULOSIS OR ACUTE GENERAL TUBERCULOSIS}

This is an acute infectious disease excited by the tubercle bacilli and characterized anatomically by the simultaneous formation of miliary tubercles in many parts of the body. The disease usually develops in early life and is secondary to some primary lesion, as a tubercular gland. The bacilli are probably disseminated by the veins. All the organs may be uniformly infiltrated with the tubercles, but more commonly certain organs, like the lungs and brain, are more affected than the others.

Symptoms.- There is debility, loss of flesh and strength, the temperature ranges between $102^{\circ} \mathrm{F}$. and $103^{\circ} \mathrm{F}$, and is characterized by an evening rise. There is cough, 
rapid respirations, and symptoms of the typhoid state are present. Tubercle bacilli are rarely found. If the lungs are chiefly affected, dyspnea, cough, and expectoration will be marked. When the intestines and the peritoneum are mainly involved, pain, distention, abdominal tenderness and diarrhea will be the most prominent symptoms.

\section{TUBERCULOSIS OF THE BRONCHIAL LYMPH-GLANDS}

This condition is frequent in childhood. The general symptoms of tuberculosis are present; namely, fever and wasting. The other symptoms are lacking.

The usual result of such an inflammation is a rupture of the gland with subsequent involvement of the lungs.

Eustace Smith's sign is supposed to be diagnostic of enlarged bronchial glands. It is demonstrated as follows: A stethoscope is placed over the large blood-vessels of the chest (at the base of the heart), with the child's head forward on the chest; then bend the child's head as far back as possible, and, if a murmur is then heard which was previously absent, enlarged bronchial glands exist. The murmur is caused by the pressure of the enlarged glands upon the vessels, the position of the head bringing them into apposition with these vessels. Bronchial glands that are not enlarged will not produce this sign.

\section{TUBERCULAR MENINGITIS}

Tubercular meningitis is fully described under diseases of the nervous system (see page I90).

\section{TUBERCULAR DISEASE OF THE BONES AND JOINTS}

Pott's disease is tuberculosis of the spine. It may be located in the cervical, dorsal, or lumbar regions of the 
spinal column. It causes a necrosis or caries of the vertebræ.

Symptoms. - The disease comes on insidiously. The earliest symptoms are due to irritation of the spinal nerve roots. Pain is the most prominent of these and is referred to various parts of the body supplied by the distribution of the nerves affected.

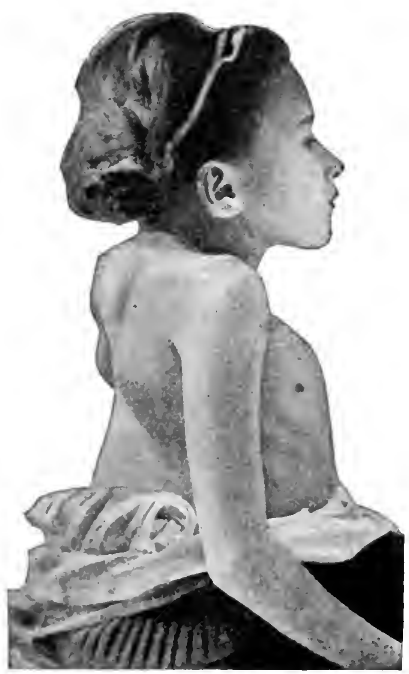

Fic. 79.-l'ott's disease of the upper dorsal vertebra. Sharp-angled kyphosis. (Clinic of von Ranke-1lerzog, Munich.)

In a short time there is a rigidity of the spine and the child assumes various postures to prevent the diseased surfaces of the vertebra from rubbing together. If he stoops to pick anything from the floor he does so without bending his back. Jumping from elevations hurts him and pulling the head away from the body relieves him. 
Soon an angular deformity called kyphosis appears at the seat of the disease in the spine. This progressively becomes worse, making a permanent deformity commonly called hunch-back.

The tubercular process causes softening of the vertebræ and abscess formation.

These abscesses vary in their location according to the seat of the lesion. In cervical Pott's disease they may be retropharyngeal; in dorsal and lumbar Pott's disease they may point in the small of the back or burrow through the sheath of the psoas muscle and point in the thigh at the lower attachment of the muscle. This is called a psoas abscess. In addition to these symptoms there is fever, wasting, pain, insomnia, and paralysis.

Prognosis.-If the children are made to rest, given proper care and attention, and their backs immobilized, a cure will result in a large number of cases.

Plaster jackets and other mechanisms are applied to keep the back rigid. Abscesses have to be opened and drained.

Coxalgia or coxitis is a tubercular inflammation of the hip-joint. It causes necrosis of the bones of the joint.

Symptoms. First Stage.-The disease begins insidiously. The first symptom noted is slight lameness or the fact that the child wears out one shoe quicker than its mate. In a short time pain develops, due to muscular spasms. The pain is referred to the knee, often causing this joint to be suspected when the hip is really at fault. These symptoms may last a few weeks or even longer.

Second Stage.-During this period the leg is in a characteristic position. The foot is everted, the thigh is 
slightly flexed and rotated outward, and the leg is apparently lengthened, due to the tilting of the pelvis.

The joint is locked from constant muscular spasm and abscesses form about the hip-joint. The duration of this stage is indefinite.

Third Stage.--In this stage the joint has been destroyed. The thigh is flexed on the abdomen, it is rotated inward, the foot is inverted, there is shortening of the leg, and a

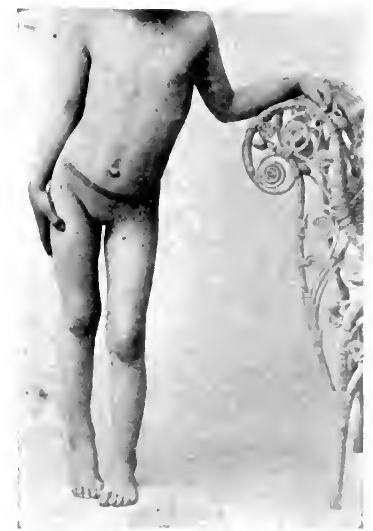

FIG. 80.-Hip-joint disease showing tilting of the pelvis in abduction, and apparent lengthening (left leg) (Moore).

curvature of the spine. Ankylosis or a permanent union of the bones may result, which prevents any movement of the head of the femur.

Treatment.-Coxalgia demands absolute rest in bed with an extension apparatus (sce Fig. I06, page 426) applied to the affected leg. This draws the bone downward so that the head of the femur does not come in contact with the acetabulum or socket of the hip-joint; 
it also immobilizes the joint. Abscesses are opened and drained.

Tubercular arthritis is a tubercular inflammation of a joint. Almost any joint in the body may be involved. The knee, ankle, and elbow are probably the most often affected.

Symptoms.-This consists of a spindle-shaped swelling of the joint without any signs of inflammation. It has a

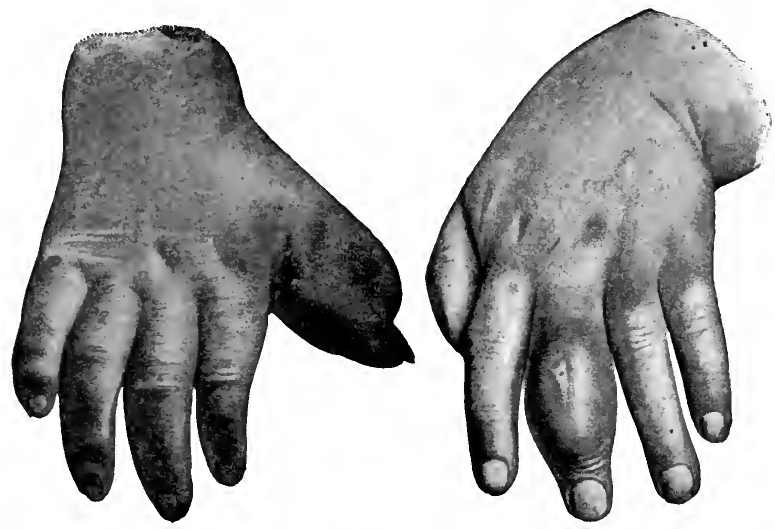

Frg. 81.-Tubercular dactylitis of the right thumb and left middle finger of a three-yearold child (Hecker, Trumpp, and Abt).

doughy feeling and a whitish appearance. It has been called white swelling. Later it may break down, the joint be destroyed, and ankylosis result.

Treatment.-This consists of immobilization of the joint by plaster casts. At times curetment is necessary.

Tubercular Osteomyelitis. - This is an inflammation of the shaft of the bone characterized by swelling and necrosis of the bone. The dead portion of the bone has to be removed. 
Epiphysitis is an inflammation of the ends of the bones.

Tubercular dactylitis is an inflammation of the bones of the hands and feet.

\section{TUBERCULAR ADENITIS}

This is seen chiefly in the cervical lymph-glands, and is characterized by swelling, softening and breaking down of the glands, sinuses resulting.

\section{TREATMENT OF TUBERCULOSIS}

Prophylaxis.-Sputum of consumptive children should be received in suitable vessels which contain antiseptic solutions, and subsequently destroyed. Phthisic mothers should not nurse their offspring. The healthy should not sleep in the apartments of those affected.

The treatment of tubercular children aims to strengthen their vitality and resisting powers and to destroy or disable the tubercular bacillus. This is brought about by good food, fresh air, frequent bathing, sunlight, avoidance of exposure, graduated exercise, a dry, wellventilated house and plenty of sleep and recreation. In bone and joint diseases absolute immobility of the part affected is essential.

Hemorrhage from the lung in childhood is infrequent. If it should occur, the child should be kept absolutely quiet and an ice-bag applied to the chest. 'The physician should be immediately notified.

Nursing.- The room should be sunny, cheerful, and well ventilated. In tuberculosis of the lungs the child should live in the fresh air. If it is too cold or stormy to stay out of doors, the windows in the nursery should frequently be raised to keep the atmosphere thoroughly 
fresh. At night the windows should be wide open, the child thoroughly covered, and not exposed to the draught. Frequent bathing and friction of the skin are essential. The bowels should be kept regular. The clothing should be warm, but the child should not be over-dressed. Exercise should be taken only upon physician's permission. All sputum should be collected in cups and disinfected.

In tuberculosis of the bones and joints the part should be kept absolute immobile. The method of applying extension will be found on page 425. Any pus from tubercular lesions should be covered with carbolic acid, I to 20 , and allowed to stand for half a day before disposal.

The diet should be wholesome. Raw eggs and milk should be taken to build up the system. Plenty of sleep and exercise without exertion are beneficial.

The sputum for examination should be collected in a sterile bottle with a wide mouth. In children under four years of age the best method to obtain sputum is as follows: Place cotton about the end of an applicator. Grasp the tongue and pass the applicator close to it back to the pharynx; this should excite a cough, when the sputum can be swabbed out, or the applicators may be placed by the child's bedside and when the nurse notices a severe attack of coughing the child is picked up and, if possible, the sputum is obtained in the same way. Another easy method of obtaining sputum is to place a large ( $\frac{1}{2}$-ounce) eye-dropper in one end of a catheter. The bulb should be compressed and the catheter passed to the pharynx. The bulb is then allowed to expand; the suction then draws the sputum into the catheter. 


\section{CHAPTER XV \\ CONTAGIOUS DISEASES}

A contagious disease is one that can be transmitted through contact with the patient.

\section{SCARLET FEVER OR SCARLATINA}

This is an acute contagious disease characterized by high fever, rapid pulse, a scarlet rash, and an unusual tendency to nephritis. The germ that causes scarlet fever has not been discovered. The contagion is carried by the clothes, bed-clothing, or other articles which have come in contact with a patient suffering from scarlet fever. Milk is suspected of sometimes being a means of dissemination. The disease can be transmitted by direct inoculation and, therefore, is characterized as a contagious disease. The poison is extremely tenacious to life, infected clothes unused for years being known to lead to fresh outbreaks. The young are especially predisposed, but not equally so. One attack practically gives immunity, as second attacks are uncommon.

Period of incubation is from a few hours to a week.

Symptoms.-Mild Cases. - At times the symptoms of scarlet fever are so mild that the disease may escape notice.

There is fever, a slight sore throat and a very faint rash, often escaping proper diagnosis; a hot bath will cause such a rash to show plainly. It may fade away very 284 
quickly and the character of the rash not be suspected until slight desquamation appears. This form is contagious and is especially dangerous, as it is often not isolated.

Ordinary Case.-The disease begins suddenly with vomiting or it may be ushered in with convulsions.

Throat Symptoms.-There is pain and difficulty in swallowing, fulness and tenderness under the jaw, and enlargement of the lymphatic glands. The tongue is at

\begin{tabular}{|c|c|c|c|c|c|c|c|c|c|c|c|c|c|c|c|}
\hline & & 1 & 2 & 3 & 4 & 3 & 6 & 7 & 8 & & 9 & 10 & $/ 1$ & 12 & 13 \\
\hline$P$. & $T$. & $7 n$. & in. $e$ & $m$ & mI. & & & $n$. & $m$ & & $e$ & mi. $e$ & m.e. & m. $e$ & \\
\hline 170 & 108 & & & & & & & & & & & & & & \\
\hline 160 & 107 & & & & & & & & & & & & & & \\
\hline 150 & 106 & & & & & & 8 & & & & & & & & \\
\hline 140 & 105 & & & 1 & $\bar{N}$ & & & II & $V$ & & & & & & \\
\hline 130 & 104 & & $v_{1}$ & & & & & & & & & $\therefore$ & & & \\
\hline 120 & 103 & & & & 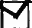 & & 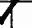 & & & & & 7 & & & \\
\hline 110 & 102 & & & & & & & & & & & & h & & \\
\hline 100 & 101 & $i$ & & & & & & & & & & & & 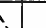 & \\
\hline 90 & 100 & $y$ & & & & & & & & & & 1 & & t. & \\
\hline 80 & 99 & & & & & & & & & & & & & & \\
\hline 70 & 98 & & & & & & & & & & & & 7 & - & \\
\hline 60 & 97 & & & & & & & & & & & & & & \\
\hline
\end{tabular}

Fig. 82.-Pulse (--.-) and temperature (-) of a simple uncomplicated scarlet fever. The acme in this case was reached somewhat late; the defervescence is rather marked (Kerr).

first heavily coated, red at the tip and edges. This white coat peels off, beginning at the edges, and in a few days it disappears and the papillæ of the tongue become bright red and swollen.

This appearance has given rise to the name strawberry tongue. The whole posterior portion of the mouth and pharynx are deeply injected and may show a punctiform erythema before the rash appears on the skin. In severe cases the tonsils may be the seat of follicular inflammation or they may be covered with false membrane. 
Eruption.-A scarlet punctiform rash appears, at the end of the first or the beginning of the second day, on the neck and chest and spreads over the entire body. Sometimes the appearance of the rash is delayed. It disappears on pressure and if the finger-nail be drawn through it a white line will remain for a second or two. It may be a uniform rash or it may appear in patches with healthy skin surrounding it.

In five or six days the rash gradually disappears and a scaly desquamation or peeling follows.

A bright rash shows a strong heart; sudden fading of the rash may mean heart failure. In some cases the rash is slightly papular or vesicular (scarlatina miliaris).

Febrile Symptoms. - The fever rises abruptly, reaching the maximum temperature of $104^{\circ} \mathrm{F}$. to $105^{\circ} \mathrm{F}$. in twentyfour or forty-eight hours, and remaining at about this height for three or four days and then falling by lysis. The duration of the febrile period is from seven to nine days. The pulse is rapid, out of all proportion to the fever, and the respirations are accelerated. The appetite is lost, the bowels are constipated, and the urine is scanty and high colored and often contains albumin.

Nervous Symptoms.-Restlessness, headache, insomnia, delirium, and convulsions may occur. Convulsions occurring late in the disease are very significant of uremia.

More Severe Cases.-Anginoid Scarlet Fever.-This form is characterized by severe throat symptoms. The tonsils are much swollen and often covered with a false membrane. The fever is high and the prostration is profound. Ulceration, and, at times, gangrene of the throat occur; the carotid artery may be involved. In this form death may result from exhaustion, aspiration pneu- 


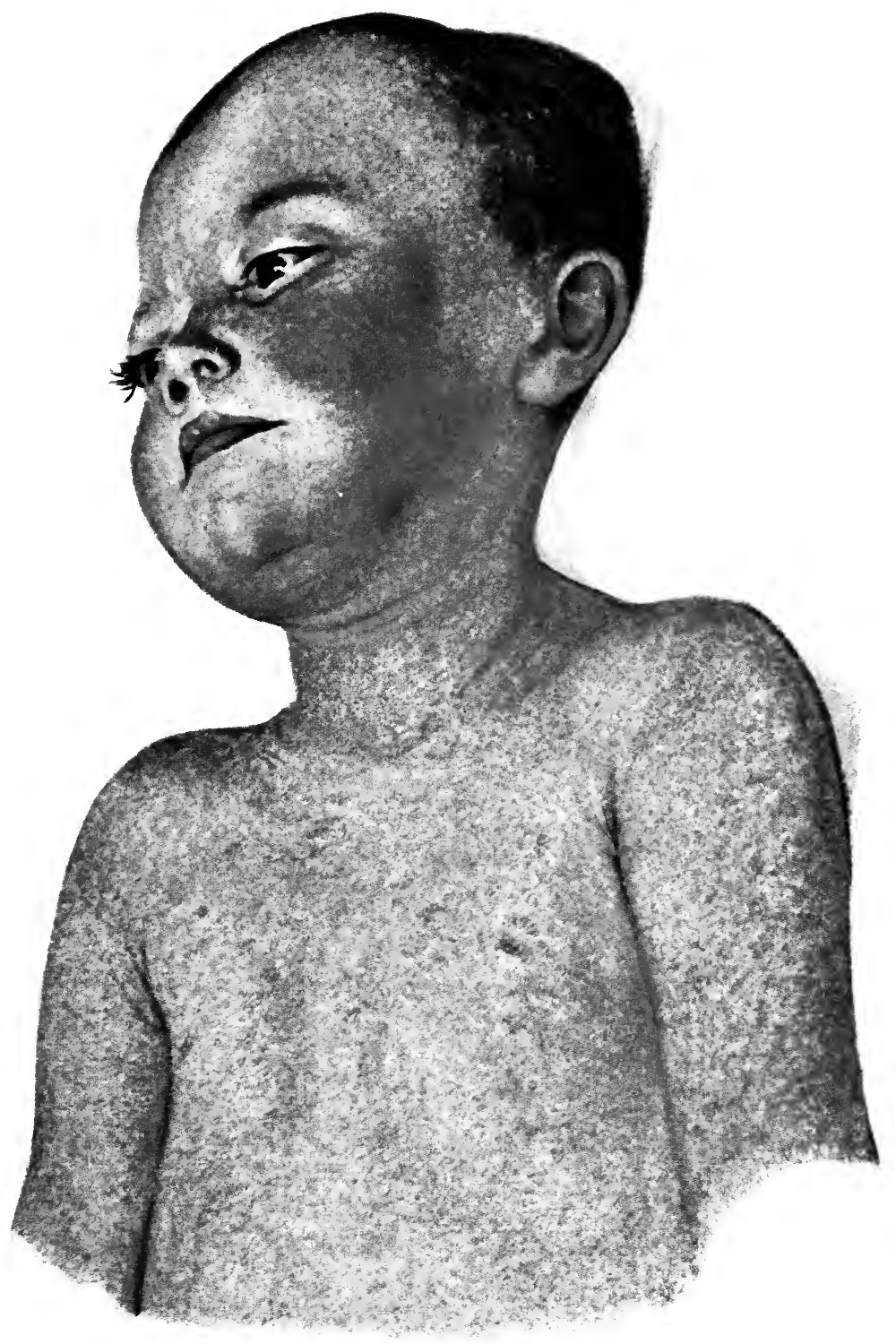

'The eruption of scarlet fever on the third day (Hecker, Trumpp, and $\mathrm{Abt}$ ). 
monia, or hemorrhage from an ulceration of the carotid artery.

Malignant Scarlet Fever.-This is a very severe form of the disease. The onset is abrupt, with a chill, vomiting or a convulsion. The fever is very high $\left(106^{\circ} \mathrm{F}\right.$. to $107^{\circ} \mathrm{F}$.). The pulse is rapid and feeble. Delirium sets in and is followed by coma. Death may result before the appearance of the rash in twenty-four or forty-eight hours. The rash, if present, may become hemorrhagic.

Complications. - Nephritis. - This usually develops during convalescence and, as it may be unattended by subjective symptoms, the urine in a case of scarlet fever should be examined daily in order to detect immediately the presence of albumin. In other cases the onset of nephritis is recognized by the suppression of urine, the development of uremia, and the appearance of dropsy.

Nephritis may be the immediate cause of death, but more commonly the case ends in recovery or in chronic nephritis.

Among other complications may be mentioned hyperpyrexia, endocarditis, pericarditis, pneumonia, suppuration of the lymphatic glands, ophthalmia, inflammation of the middle ear, chorea, and a peculiar inflammation of the joints resembling rheumatism.

Prognosis.-Always guarded. The mortality varies in different epidemics from five to forty per cent.

Treatment and Nursing.-A case of scarlet fever should be immediately isolated. It is kept in isolation for at least six weeks, for it takes that length of time for the peeling to be completed. Children should not mingle with others for a month following their release from quarantine and should not sleep with others for three months. 
Cases of scarlet fever should be kept absolutely at rest to avoid complications and should be given a liquid diet as long as the fever lasts.

The rash and the peeling which follows render it necessary to anoint the surface of the body with cold cream or carbolized vaselin two or three times a day. This relieves the itching and irritation of the skin and controls the desquamation.

To avoid the danger of nephritis the children should be encouraged to drink water or lemonade freely.

The nose and throat should be sprayed with antiseptic solutions. Nervous symptoms are relieved with ice-caps and cool sponges. Cardiac weakness should be combated by heart stimulants.

Isolation and Disinfection in Contagious Cases.-Prophylaxis should be complete, as the disease is highly contagious and is prone to leave many serious complications. The room selected should be at the top of the house if practicable, and it should have plenty of ventilation and be bright and sunshiny. All upholstered furniture should be removed, curtains and hangings taken down, and the carpets taken up.

Where possible, two rooms and a bath should be set aside for the nurse and the patient. They must be isolated from the rest of the house, and no one should be allowed in the room except the physician and the nurses, unless he gives permission.

The nurse should not eat her meals in the room with the patient.

The room should be wiped up daily with a duster moistened with carbolic, bichlorid, or a 2 per cent. formalin solutions. The floor should be swept with a broom 


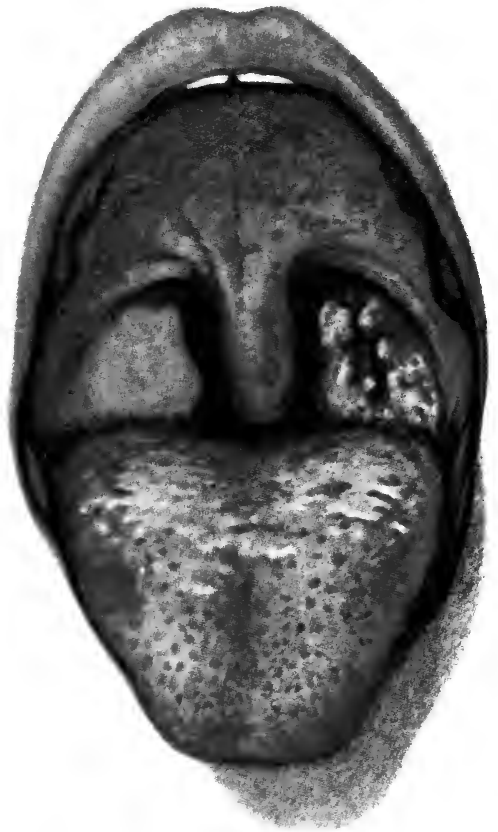

Scarlatinal angina (third day) (Hecker, Trumpp, and Abt).

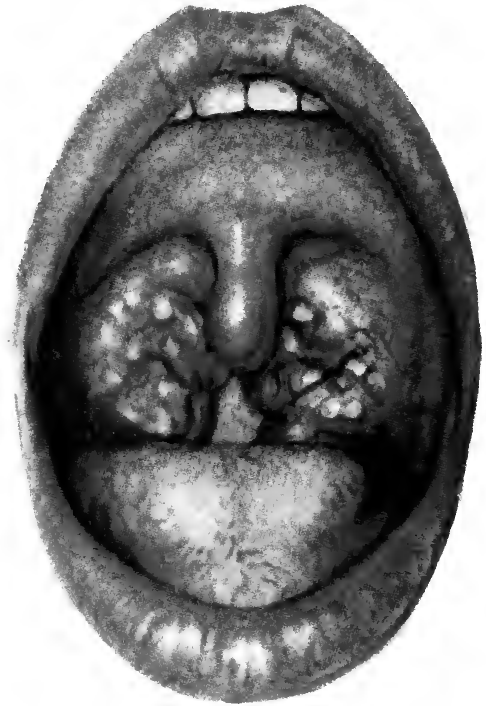

Follicular tonsillitis (Hecker, Trumpp, and Abt). 
covered with a duster also moistened with the disinfecting solutions. After use, all dusters should be thoroughly soaked in disinfectant and then washed.

The dishes and linen should be placed in separate metallic vessels containing water; these vessels should be draped in sheets wet with disinfecting solution. They should be removed daily.

Unused food can be put into a similar receptacle, which should be removed three times a day. The contents should be burnt.

Sheets wet with carbolic acid, I:40, should be hung over the doorways. All desquamation should be immediately burned or immersed in carbolic acid. The thorough disinfection of all articles which come in contact with the child is absolutely necessary. The nurse should be protected by a gown and cap, and before going out should take an antiseptic bath (see page 435) and change all of her clothing. The physician should be protected by a gown and cap while in the room and before leaving should wash his face and hands in an antiseptic solution.

The contagium is contained in the secretions such as the urine, bowel movements, perspiration, and discharges from the nose and ear. All of these should be disinfected by covering with carbolic acid, I:40. The mildest cases should receive the same treatment and care, and isolation should be for the same length of time.

The stools should be received in a vessel containing a disinfectant. An equal quantity of disinfectant to the size of the excreta should be added, the whole thoroughly mixed, and allowed to stand for a half-hour before emptying into the water-closet hopper. The bed-pan should contain disinfectant when not in use. It should be thoroughly 
rinsed in warm water before placing it beneath the child, otherwise the disinfectant might burn the buttocks.

After recovery the child should be given a warm bath and shampoo with bichlorid, $\mathrm{r}: 5000$, rolled in a clean sheet which has not been in the isolation rooms, and carried to another room, where he can be dressed.

The rooms should then be sealed, all articles in the isolation rooms hung over lines, and the rooms fumigated with formaldehyd.

The disinfection of the nurse is practically the same as for the patient.

A discharging nose or ear may be capable of causing the disease after the desquamation has ceased. Cases of empyema following scarlatina have caused outbreaks in surgical wards.

\section{MEASLES OR RUBEOLA}

This is an acute contagious disease characterized by catarrh of the respiratory tract, moderate fever, and a papular eruption appearing on the fourth day, lasting two or three days, and disappearing by fine desquamation. The rash also has the tendency to form crescents.

Measles is a highly contagious disease. The poison is transmitted through the clothing and other articles which have come in contact with the person suffering from the disease; it can also be contracted by direct contact.

The contagium is apparently associated with the nasal and bronchial secretions, but has not been isolated.

Measles is most commonly observed in children, but unprotected adults are very liable to be attacked. It is essentially an epidemic disease, but now and then sporadic cases are seen. One attack is fairly protective, but does not give absolute immunity. 


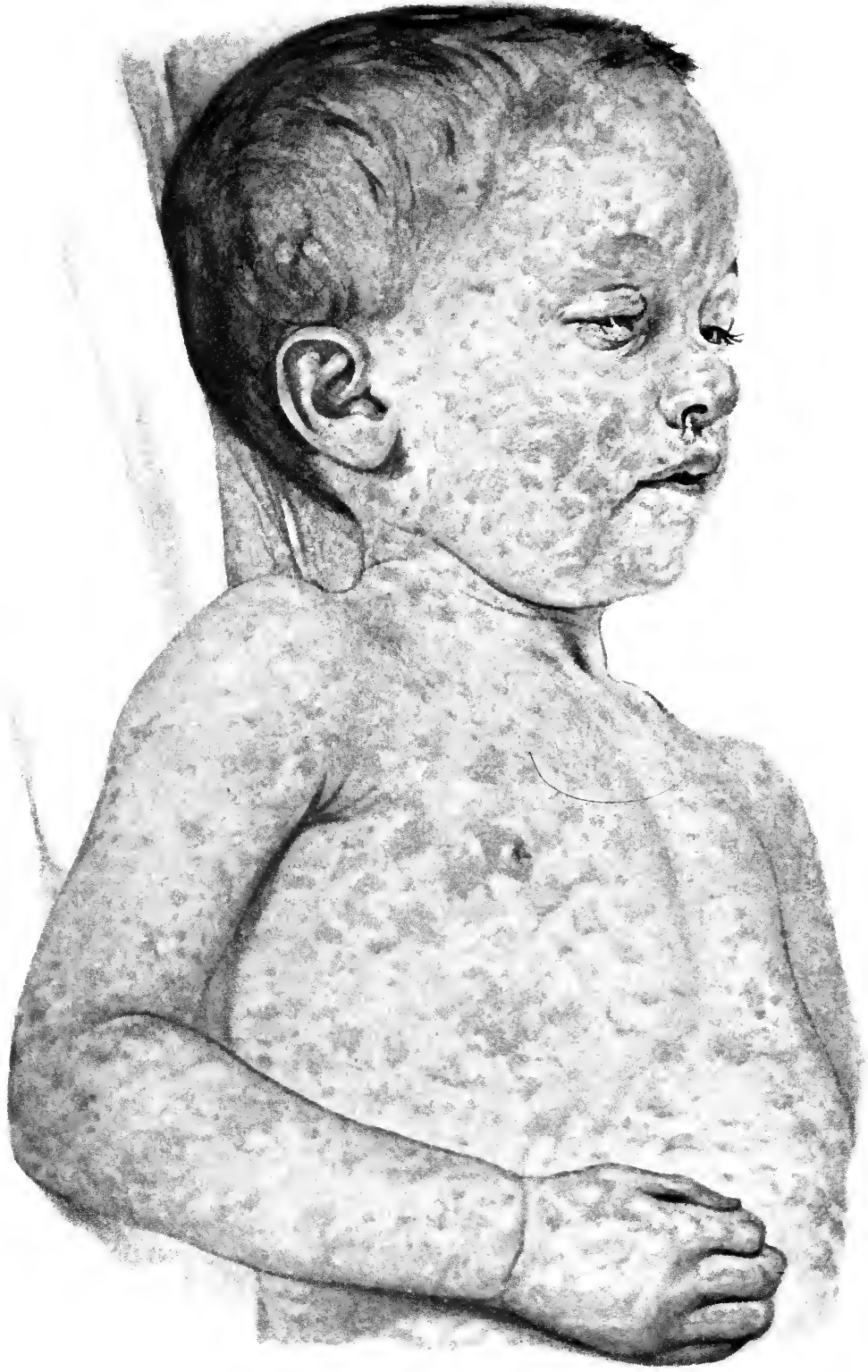

The eruption of measles two days after its first appearance (Hecker, Trumpp, and Abt). 

The period of incubation is two weeks.

Symptoms.-Prodromal.-There is chilliness, coryza, watering of the eyes, photophobia (the inability to stand light), cough, and drowsiness.

The Fever.-The temperature rises rapidly to $102^{\circ} \mathrm{F}$. or I0 $3^{\circ} \mathrm{F}$., but on the second day there is decided remission, the temperature remaining down until the appearance of the rash on the fourth day when it again rises to or beyond the first range of temperature. It remains at this height for two or three days and then falls by crisis.

\begin{tabular}{|c|c|c|c|c|c|c|c|c|c|c|c|c|c|c|c|c|}
\hline \multirow{2}{*}{ FAHR. } & \multicolumn{2}{|c|}{1} & \multicolumn{2}{|c|}{2} & \multicolumn{2}{|c|}{3} & \multicolumn{2}{|c|}{4} & \multicolumn{2}{|c|}{5} & \multicolumn{2}{|c|}{6} & \multicolumn{2}{|c|}{7} & \multicolumn{2}{|c|}{8} \\
\hline & $M$. & $E$. & $M$. & E. & $M$. & E. & $M$. & E. & $M$. & E. & $M$. & $E$ & $M$ & $E$. & $M$. & $E$ \\
\hline 107 & & & & & & & & & & & & & & & & \\
\hline 106 & & & & & & & & & & & & & & & & \\
\hline 105 & & & & & & & & & & & & & & & & \\
\hline 104 & & & & & & & & & & & & & & & & \\
\hline 103 & & $A$ & & & & & & & & & & & & & & \\
\hline 102 & & & & & & & & & & & & & & & & \\
\hline 101 & & & & $\wedge$ & & & & & & & & & & & & \\
\hline 100 & & & $V$ & & $V$ & & & & & & & & 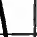 & & & \\
\hline 99 & & & & & & & & & & & & & & & & \\
\hline 98 & & & & & & & & & & & & & $V$ & & 1 & \\
\hline 97 & & & & & & & & & & & & & & & & \\
\hline & & at & 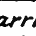 & hal & $S t$ & & & $x a$ & athe & & & $15 c$ & & ge. & Cor & \\
\hline
\end{tabular}

FiG. 83.-Temperature chart of rubeola of moderate severity in a child of four and one-half years (Kerr).

The Catarrh.-There is redness of the conjunctiva, lacrimation, sneezing, hoarseness, cough, and expectoration. There may be vomiting and diarrhea. A slight adenitis of the cervical glands is common.

Rash.-This appears on the fourth day on the face and rapidly spreads over the entire body. It is composed of 
small, dark red, velvety papules which form groups having crescentic borders. There is an eruption on the mucous membrane of the throat one day before the rash appears on the skin.

In two or three days the eruption begins to fade and a fine desquamation soon follows.

Koplik's Sign.-This consists in minute bluish-white specks surrounded by a red areola, appearing on the mucous membrane lining the cheeks and lips one or two days before the rash appears.

Malignant or hemorrhagic measles is a form of the disease which occurs under bad hygienic conditions and is characterized by a petechial rash, hemorrhages from the mucous membranes, and by profound prostration.

Complications and Sequelæ.-By far the most prevalent are capillary bronchitis or catarrhal pneumonia and otitis media. Gastro-intestinal disturbances, cancrum oris, tuberculosis, and paralysis are also seen.

Prognosis.-Guardedly favorable. Complications are apt to occur and render the prognosis grave. The usual mortality is low. In epidemics and in hospitals at times it is as high as 30 or 40 per cent. The majority of the deaths are due to bronchopneumonia.

Treatment and Nursing.-Measles must be isolated. The quarantine is not so rigid as in scarlet fever, for sixteen days only is the length required. A dark room must be chosen on account of the photophobia, or intolerence to light.

The methods for isolation and disinfection are described on page 288 .

The treatment is symptomatic. Inunctions of carbolized vaselin are used to allay the irritation and help the des- 


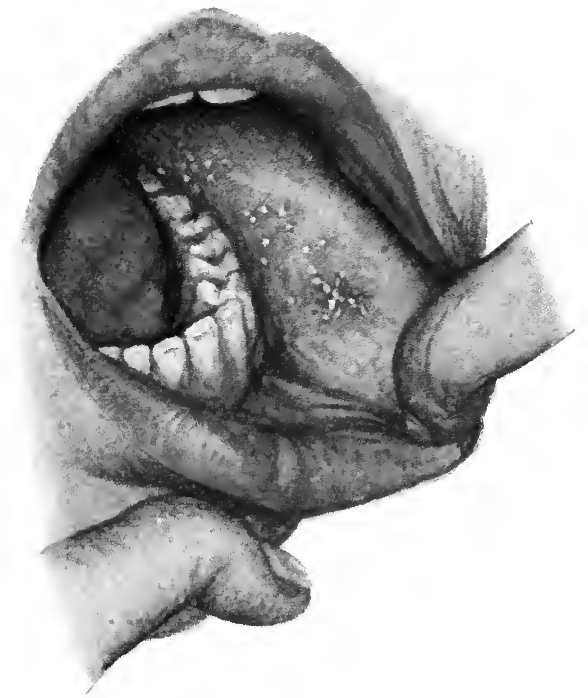

Koplik's spots in measles (Hecker, Trumpp, and Abt).

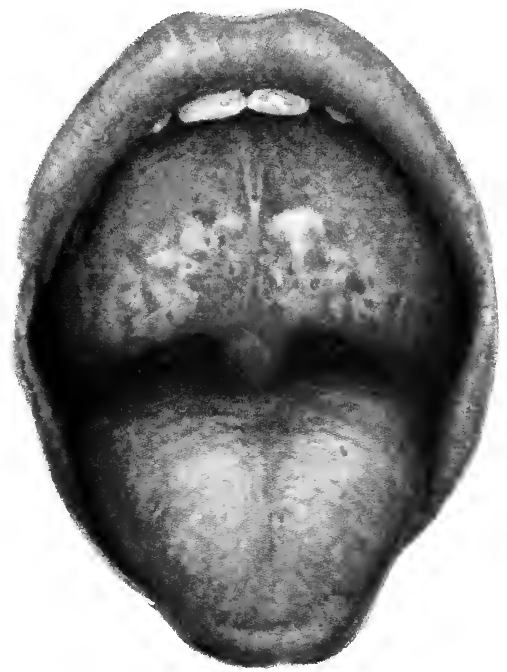

Appearance of the throat in measles (Hecker, Trumpp, and Abt). 

quamation. Warm baths are given after the rash disappears to facilitate desquamation.

The most important complications to guard against in measles are bronchopneumonia and otitis media. To avoid these children should remain in bed and a flannel or cotton jacket should be worn. The chest should be rubbed daily with some counterirritant, such as camphorated oil or amber oil. Earache should be treated by syringing the ear with hot water (see pages $4 \mathrm{rO}$ and $4 \mathrm{II}$ ).

It is advisable for the child to wear some form of protection over the ears throughout the attack. A simple expedient is to place cotton in the external auditory meatus and cover the head and ears with a snugly fitting cap.

The nose and throat must be frequently cleansed with antiseptics or normal salt solution.

\section{RÖTHELN OR RUBELLA}

This is an acute contagious disease resembling both scarlet fever and measles, but differing from both in its short course, slight fever, and freedom from sequelæ. The disease is highly contagious; the poison may be carried in the clothes or may be directly transmitted.

Symptoms.-Prodromal symptoms are slight or absent. The disease begins with drowsiness, slight fever, and sore throat. The eruption appears on the first or second day and varies greatly in character. In some cases the rash is composed of small, red, slightly elevated papules resembling measles; in others the rash is bright red and diffuse, resembling scarlet fever. It begins on the face and spreads rapidly over the entire body, but it fades so quickly that the face may be clear before the extremities become involved. Slight desquamation frequently follows, though 
it is often absent. Apart from the sore throat the catarrhal symptoms are slight. The superficial cervical and posterior auricular lymph-glands are more swollen than in measles. The duration is from three to five days.

The prognosis is good. Isolation for five or six days is advisable, though not imperative.

Nursing.-The disease does not require much attention. The room should be darkened, the nose and throat washed with antiseptic sprays, and the temperature, pulse, and respiration taken twice a day.

\section{DIPHTHERIA}

This is an acute contagious disease excited by the Klebs-Löffler bacillus and characterized by moderate fever, great prostration, glandular enlargement, and a fibrinous exudate which is usually located in the throat.

Etiology.-Diphtheria is most common between the ages of three and six in children who suffer from catarrhal conditions of the nose and throat. The poison is contained in the secretions of the throat and may be transmitted through the atmosphere and through the clothing. One attack does not protect the child from a second. The Klebs-Löffler bacillus is found in the membranous exudate and the constitutional symptoms result from the poison generated by this bacillus.

The membrane is not a true membrane, but a necrosis of the superficial cells of the mucous membrane caused by the Klebs-Löfler bacillus. It is grayish-white in appearance and more or less adherent, so that when it is stripped off it leaves a raw, bleeding surface. Sometimes the necrosis extends to the deeper tissues, causing widespread ulceration and gangrene. The membrane is 
usually found on the tonsils, pillars, and pharynx, but it may extend to the mouth, larnyx, and nose. It is then called laryngeal diphtheria or membranous croup, and nasal diphtheria, respectively. The lymphatic glands are considerably swollen; the spleen is engorged. The lungs frequently show bronchopneumonia.

Types.-According to location, there may be faucial, laryngeal, nasal, and cutaneous diphtheria. According to the severity of the attack the disease may be mild, grave, and malignant.

The period of incubation is from two to ten days. A condition, resembling faucial diphtheria, called Vincent's angina has already been described on page II2.

Symptoms.-Catarrhal diphtheria, so-called because there is an absence of membrane, is only a catarrhal condition of the nose and throat; the secretions, however, contain the Klebs-Löffler bacillus. The symptoms are mild, but the risk of spreading the contagion is great.

Faucial Diphtheria of Ordinary Severity.-The disease commonly begins with a chill, moderate fever, malaise, and sore throat. The fever, as a rule, is not very high, $102^{\circ} \mathrm{F}$. to $104^{\circ} \mathrm{F}$, and its course is quite irregular. The pulse soon becomes rapid and feeble, the bowels are constipated, the urine is scanty and frequently albuminous, and the prostration is out of proportion to the severity of the febrile symptoms.

Local Phenomena of Faucial Diphtheria.-The child complains of difficulty in swallowing, the muscles of the neck feel stiff, and there is tenderness under the jaw. The lymphatic glands are considerably enlarged and the tonsils are covered with grayish-white membrane which, when stripped off, leaves a raw, bleeding surface. The 
membrane soon forms again and may extend to the larynx and into the nose. The average duration of the disease is from one to two weeks.

Laryngeal Diphtheria.-This is usually secondary, by extension from the faucial diphtheria, but it may be primary. It is recognized by hoarseness, aphonia (loss of voice), croupy cough, progressive dyspnea, and stridulant breathing. The wings of the nose play, the sternomastoid muscles are prominent, and other signs of dyspnea are present. Shreds of false membrane are sometimes expectorated during violent fits of coughing. The febrile symptoms are usually slight. Death often results from suffocation, but recovery is not impossible in the most unpromising cases. Intubation is necessary at times (see page 302).

Nasal Diphtheria.-This is nearly always secondary. It is recognized by an offensive discharge from the nose, epistaxis, and excoriation of the lips and wings of the nose. The false membrane may be detected within the nasal chambers upon inspection.

Cutaneous Diphtheria.-This may be primary or secondary. The constitutional symptoms are similar to those of faucial diphtheria. The membrane may appear at any point where there is excoriation.

Complications.-Capillary bronchitis, pneumonia, myocarditis, otitis media, nephritis, and paralysis. The most prevalent are bronchopneumonia, nephritis, and postdiphtheritic paralysis, the latter generally occurring during convalescence and being observed in about 15 per cent. of all cases.

Post-diphtheritic Paralysis.-There is no relation between the severity of the attack of diphtheria and the 


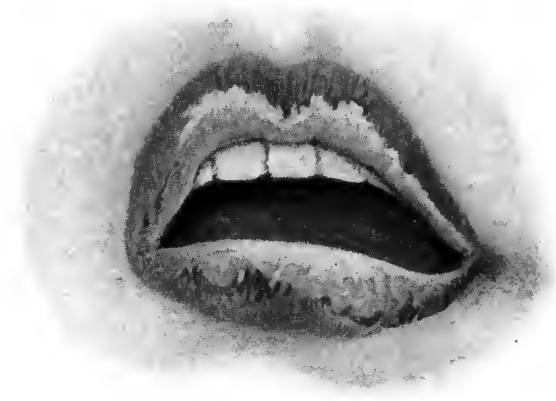

Diphtheria of the lips (Hecker, Trumpp, and Abt).

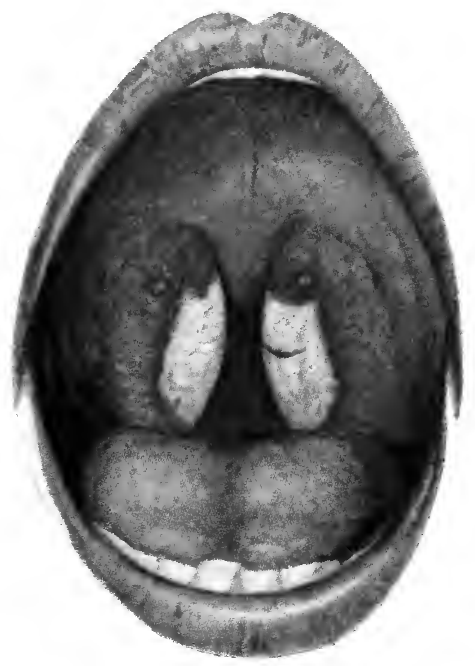

Pharyngeal diphtheria (Hecker, Trumpp, and Abt). 

liability to paralysis. Mild cases which are thought to be simple pharyngitis at times are followed by troublesome paralysis. The pharynx is usually the principal seat of the paralysis, which can be recognized by difficult swallowing and imperfect speech, and frequently the regurgitation through the nose of liquids. Next in frequency the eyes are involved and there is strabismus (cross-eyes), and ptosis (dropping of the upper lids). The heart may be affected and in such cases, if death does not immediately result, there may be a remarkable slowing of the pulse. In some cases there is an extensive involvement of the extremities. Recovery from the paralysis usually occurs.

Prognosis.-Always guarded. The mortality of diphtheria is from ro to 50 per cent. In true faucial diphtheria the prognosis is usually good. In laryngeal and nasal it is grave. Three-fourths of the cases which end fatally die of exhaustion.

Treatment.-Prophylaxis.-In no other disease are prophylactic measures so imperative as in diphtheria. All cases of recognized diphtheria, as well as all cases of suspected diphtheria, should be immediately isolated. Other children in the family should not be allowed to attend school. The suspected cases of diphtheria should be kept in isolation until two or three negative cultures are obtained. If diphtheria is not present this means only two or three days' isolation.

In true cases of diphtheria the quarantine should be kept up until a negative culture is obtained, after the throat symptoms are well. This is usually in from ten days to two weeks.

At times it is necesssary for the nurse to obtain the 
culture from a case of diphtheria. To do this she must follow the instructions as given on page 39 .

Special attention should be paid to all attacks of croup in children, especially to those attacks which do not come on suddenly in the night. It may be laryngeal diphtheria.

Do not examine an infant's throat immediately after feeding.

Antitoxin is obtained from selected horses. The method by which it is produced is as follows: The horse is given a mild case of diphtheria by injecting a few KlebsLöffler bacilli into its system. The horse recovers from the attack. A second attack is induced by the same methods. It takes a larger number of bacilli to cause a reaction than it did at first, showing that a certain resistance to the poison has developed in the blood of the horse. Other injections are made, each more powerful than the preceding, and are continued until the horse fails to develop any symptoms of diphtheria, even though doses are used which would have killed him at first. This proves that the blood contains sufficient resistance to the poison or the toxin of diphtheria to destroy the bacilli as soon as they enter the system. The blood is then withdrawn, and the scrum alone is used. It is standardized and called antitoxin, because it contains the principle which overcomes the toxin of diphtheria.

The only annoying symptom attending its administration is the appearance of hives.

The reason for giving antitoxin is to throw into the human system at once a large amount of resistance to the growth and development of the Klebs-Löffler bacilli. The same resistance or antitoxin is manufactured in the human system, but it takes days for it to become of suff- 
cient strength to combat the bacilli which are developing with almost equal rapidity. The immense advantage of throwing fully developed antitoxin into the system to combat the early and unorganized attack of the bacilli is

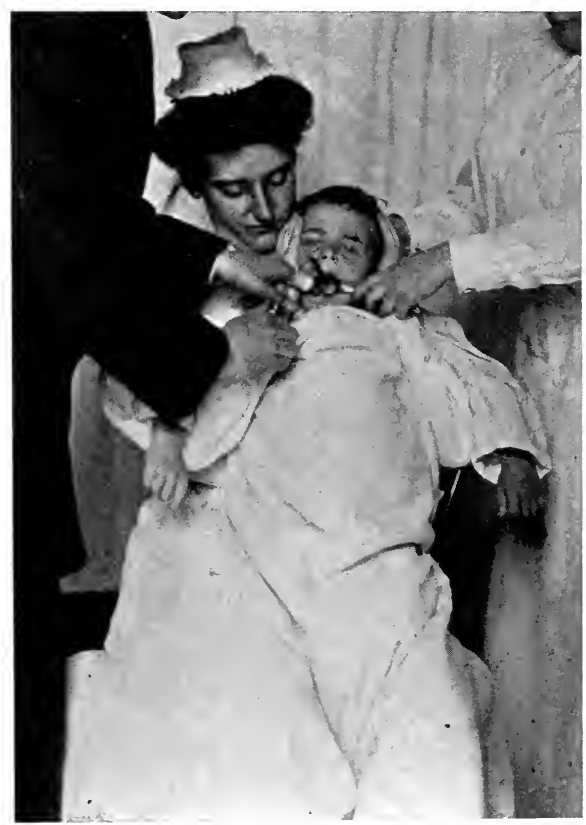

Fic. 84.-Position for intubation. The child's legs are wrapped in a blanket, and grasped between knees of nurse. Her arms are passed beneath child's and her hands fix the head.

evident. It is like having a standing army to quell incipient revolts.

To obtain the best results large quantities of antitoxin should be used early in the disease; even before the diagnosis is made in suspicious cases. If no improvement 
is noticed within twelve hours the dose is repeated. The dose of antitoxin for children is from 2000 to 4000 units. It is given hypodermically. It is influenced by the weight of the child; heavier children require larger doses.

Nursing.- The room should be selected as in scarlet fever, and the methods for isolating and nursing contagious

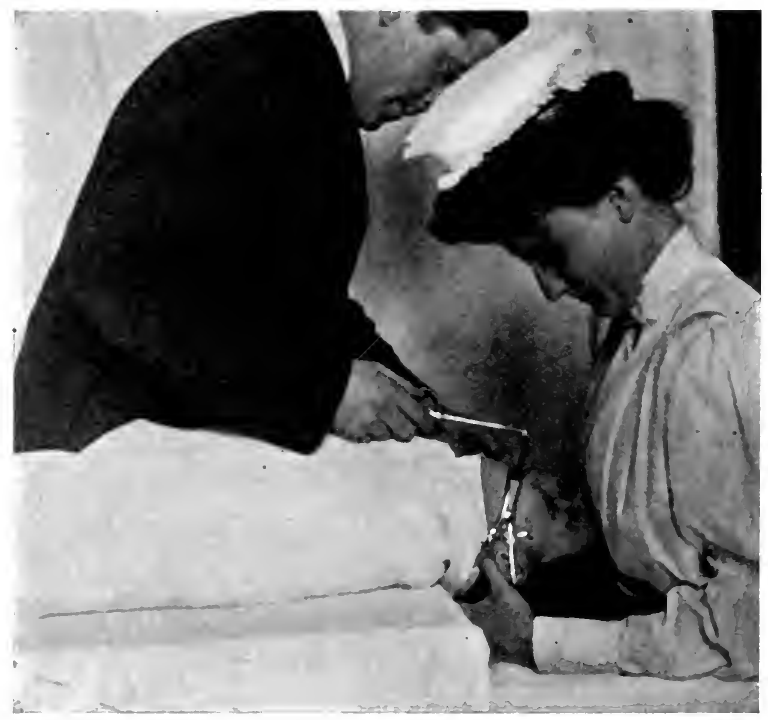

Fig. 85.-Position for intubation. The child's head is allowed to fall backward and is firmly held in position lehind edge of table.

cases as described on page 288 should be followed. A moist atmosphere should be maintained.

All cases which have been exposed to the contagium should be immunized with about rooo units of antitoxin; this immunity will last for about one month.

The nurse in charge of the case should also receive an 
immunizing dose of antitoxin. As the contagion is not contracted through the atmosphere of the room or through the air we breath, but through the discharges from the patient's nasopharynx, care should be taken in this direction. The hands should be thoroughly washed and immersed in carbolic-acid solution, and all instruments which are used in connection with the patient should be

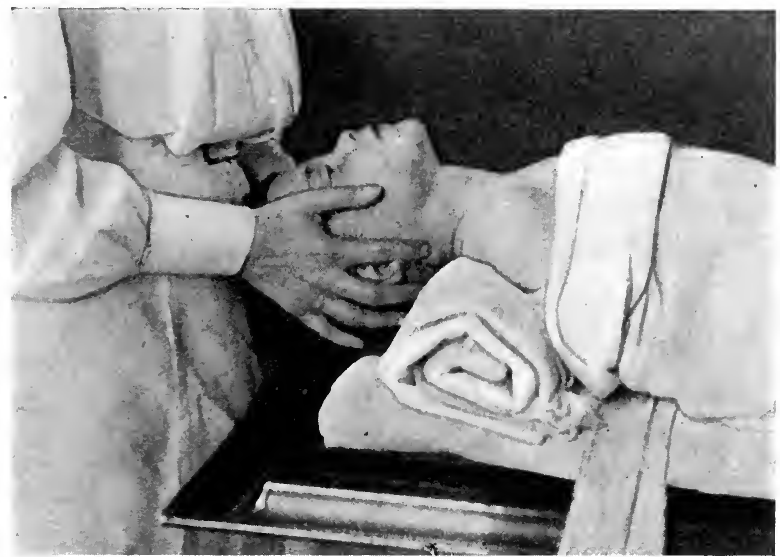

Fig. 86.-Position for tracheotomy. The rolled blanket beneath shoulders and neck makes trachea prominent.

immersed in the same solution. For this purpose a basin of carbolic solution, $1: 40$, should be kept constantly in the room. All linen and gauze which has been contaminated by the discharges of the patient should be immediately sterilized or burned. No one but the physician should be allowed in the room and he should be protected by cap and gown. The nurse should not leave the sick-room without changing her garments and washing thoroughly in carbolic or bichlorid solution. The nurse should 
spray her nose and throat three or four times a day with some antiseptic solution, and when out should not visit any houses, particularly where there are children. After a patient is well, all articles should be thoroughly fumigated and the patient should receive two disinfectant baths.

Irrigation of nose with normal salt solution from a fountain syringe is often used in cases of nasal diphtheria.

In faucial diphtheria the spraying of the throat with antiseptic or normal salt solution must be thoroughly done. In cases where the children are prone to eject pieces of membrane during irrigation, it is well for the nurse to protect her eyes with glasses and tie a piece of gauze over her mouth and nose. She should thoroughly disinfect her person, the bed, or the floor if they become contaminated with discharges.

The pulse should be under observation at frequent intervals throughout the attack and during convalescence.

Intubation.-In laryngeal diphtheria it is necessary at times to perform intubation. The operation consists in the introduction of a tube into the larynx. It opens the larynx and allows free breathing in cases where the larynx has become almost closed from the diphtheritic membrane.

Different caliber tubes are used for the various ages. During the operation it is necessary for the nurse to hold the child's head in the following manner: The child is wrapped in a blanket to secure the arms and legs. The nurse's legs being crossed, her knees should firmly grasp the child's legs, her arms should hold the child's upper extremities, and her hands fix the head. At times the child is placed on a table, the head extending over the end and firmly held by a nurse. The child should be wrapped in a blanket. 
Tracheotomy.-An incision of the trachea. It is necessary at times to open the trachea below the larynx to save the child from suffocation. A tube is introduced through the opening and the breath is drawn into the lungs through this passage.

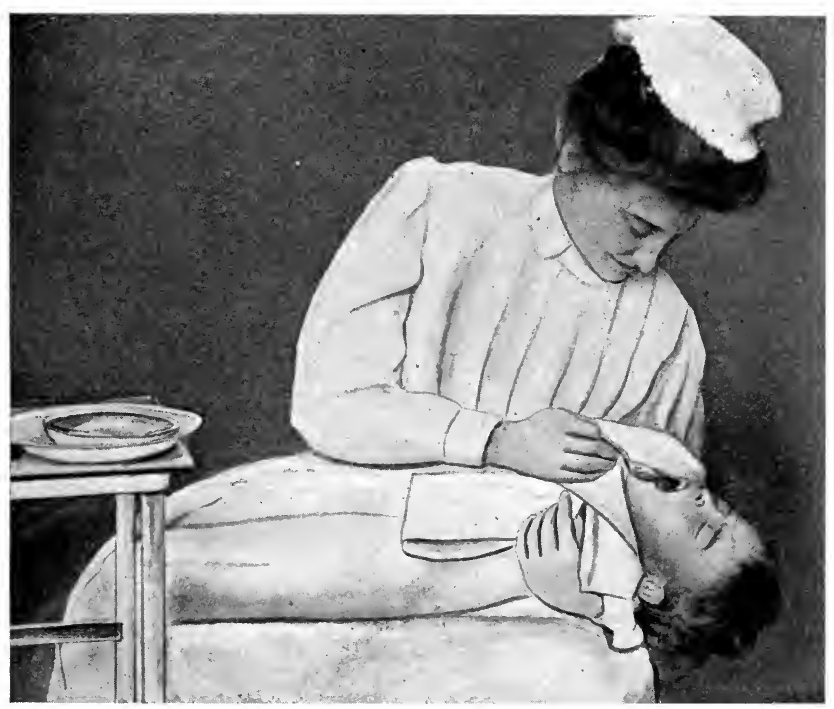

FIG. 87.-Position for feeding in intubation. The head is allowed to drop over side of lap. Junket or semifluid food is most easily swallowed.

Nursing.- Intubation and tracheotomy demand constant nursing and watching. In tracheotomy if a piece of membrane or mucus plugs up the openings in the tubes suffocation results. If it becomes necessary to remove the inner tracheotomy tube, it is unlocked and drawn out; it should be cleansed in boric acid. Long feathers are passed through the opening and the mucus dislodged. 
After the air-passages are free the inner tube is reinserted. A moist atmosphere should be maintained. After the attack is over the tube is removed, the opening closed, and the child resumes breathing through the natural passages.

In intubation, after the physician has introduced the tube, he may cut the silk thread immediately or he may loop it over the ear. If he does the latter, it must be held fast by a strip of adhesive plaster. It is also wise to tie the children's arms in such a way that they cannot reach the string and pull out the tube; cuffs may answer this purpose (see page 434).

When the silk thread is to be removed, always cut the knot off first. If this is not done, the wrong end may be pulled and the knot will then catch in the hole through which the thread passes, causing the tube to be dislodged.

Feeding in Intubation. - The child should be placed on its back across the nurse's lap and the head allowed to drop slightly lower than the body. This allows the food to pass into the pharynx and not into the larynx. Semiliquids, such as gruels, junket, thickened broths, etc., are more easily swallowed by older children than liquid food.

\section{SMALLPOX OR VARIOLA}

This is an acute contagious disease characterized by an eruption which is at first papular, then vesicular, and finally pustular.

Etiology.-The poison of smallpox is extremely tenacious to life, remaining alive in clothes for a long time, and another attack of the disease may start from this source. Unless protected by vaccination or a previous attack the whole race is susceptible, from the child in utero to the very aged. The negro race is especially prone to the disease. 
Symptoms. - The disease usuully begins with a chill followed by vomiting and intense lumbar pains. The fever rises rapidly, reaching $104^{\circ}$ to $105^{\circ} \mathrm{F}$. in twenty-four hours. It continues at this height until about the end of the third or fourth day, when it drops several degrees. It remains at this new level until the vesicles become pustules, when a hectic fever develops which may be higher than the original temperature, and like all hectic temperatures it is marked by wide fluctuations. The temperature finally falls by lysis about the eighteenth day of the disease. All the symptoms which attend fever are present.

The eruption is first noticed about the third or fourth day, appearing as small red spots on the face, forehead, and wrists. These small red spots are rapidly converted into small round papules which feel like shot under the skin. This eruption rapidly spreads over the entire body. About the third day of the eruption the papules are converted into clear vesicles, divided by small filaments into several parts, something like the divisions of an orange. This is termed loculation. When they are pricked with a needle the entire contents is not discharged, but only the fluid in the divisions opened is obtained. The vesicles have a small impression at their summit which is termed umbilication, and are surrounded by a red ring of inflammation.

In two or three days the fluid in the vesicles becomes turbid and they become pustules. The loculation and the umbilication disappear. Between the lesions the skin is swollen and edematous, so that the features are unrecognizable. In three days more the pustules rupture, soft yellow crusts form which have an offensive odor, and 
adhere to the skin for a week or more. When the crusts fall off, pockmarks (small scars) remain as a permanent deformity.

Confluent smallpox is a severe form of variola in which the pustules coalesce and the symptoms are severe.

Malignant smallpox is associated with hemorrhages; at times there is bleeding into the pustules, constituting what is sometimes called black smallpox.

Varioloid is a modified smallpox occurring in one who has been previously protected by vaccination.

Prophylaxis.-Universal vaccination.

Treatment.-The child should be immediately isolated. The room should be selected and the same precautions practised as described under Scarlet Fever (sce page 288). The treatment is symptomatic.

Nursing.-The instructions for isolating and disinfecting in contagious cases as described on page 288 must be followed.

The temperature of the room should be maintained at $68^{\circ} \mathrm{F}$. It should be darkened to prevent pitting as much as possible. The diet should be liquid, and plenty of water or lemonade given at frequent intervals.

It is absolutely necessary that the nurse should have been successfully vaccinated before attending a case of smallpox. If she has been exposed to the disease while unprotected by vaccination she should be immediately inoculated. All others who have come in contact with the disease should likewise be immediately vaccinated. She should not go out without taking a full bichlorid bath, and then should not visit other families. The child's urine should be examined frequently and the temperature, pulse, and respiration taken every three hours. 
f.

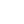

3

$=$

$\because$

$?$

-

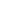

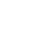




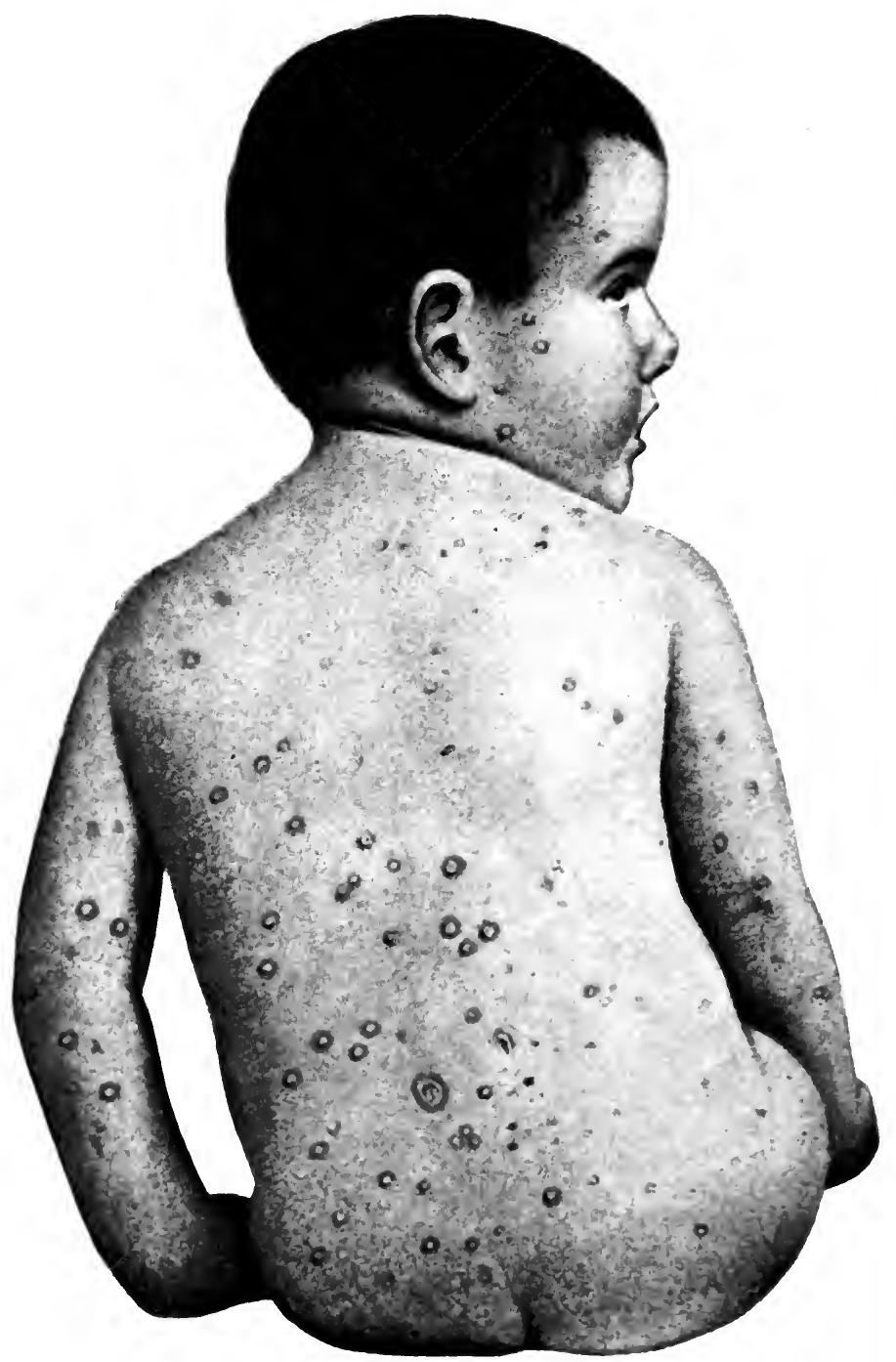

The cruption of varicella on the fourth day (IIecker, Trumpl, and $A \mid t)$. 
Itching may be relieved by frequent sponging and soaking the crusts with oil. The child's hands should be covered with mittens, preferably wet with normal salt solution. The children should not be permitted to scratch themselves; cuffs may be necessary (see page 434). The eyes should be frequently syringed, and the nose and throat sprayed.

To prevent marked pitting: (I) The vesicles should be broken up with a fine sterile needle. (2) The base of the vesicles, after they are broken, should be cauterized with a sharp stick of silver nitrate. (3) The papules should pe painted with iodin. (4) Light and air should be excluded by covering the exposed skin surface with sweet oil and dusting upon this a powder composed of equal parts of bismuth subnitrate and prepared chalk twice daily. This forms a mask.

\section{VARICELLA OR CHICKEN-POX}

An acute contagious disease of short duration characterized by slight fever and a vesicular eruption which disappears in two or three days.

Symptoms.-There is slight fever and before the end of the first twenty-four hours there is an eruption usually appearing on the face and chest. At first the eruption consists of widely scattered papules which soon become vesicles. The vesicles are superficial. They are neither umbilicated nor loculated and usually are not surrounded by a red inflammatory ring. At times a small red areola occurs. The eruption appears in crops lasting over two or three days and rarely pustulates or leaves scars. The vesicles dry up and form crusts which adhere for a few days. Sometimes the drying starts at the center and thus gives the appearance of umbilication. 
Erysipelas occasionally complicates the disease.

Treatment.-The disease is contagious, but it rarely requires isolation. As long as the crusts are present the disease can be transmitted.

Nursing.-For the itching, mild solutions of carbolic acids can be applied. To remove the crusts nothing is better than applications of ichthyol ointment.

Mittens should be placed on the child's hands to prevent them from scratching themselves.

\section{VACCINIA (COWPOX)}

Cowpox is a general disease with a local manifestation acquired by vaccination.

\section{VACCINATION}

The value of vaccination was first shown by Edward Jenner, in 1798 . He noticed that the dairymen who came in contact with the disease in cattle were rarely affected by smallpox. At that time smallpox was universal, the person who was not pock-marked being the exception, for terrible epidemics depopulated towns and villages. Jenner reasoned that cowpox had rendered these men immune to smallpox; therefore, arbitrary inoculation of individuals with the scabs from affected cows would produce the same immunity in others. He was fortunate in establishing his claims and soon vaccination became a fixed custom.

To realize the immense benefit it has been to the human race it is only necessary to compare existing conditions with those of a hundred years ago. Now the pock-marked individual is the exception and epidemics are controlled. Antivaccination societies reason ignorantly, confuse 
syphilis, sometimes called pox, with vaccination, exhibiting pictures of syphilitic ulcers as the dire results of vaccination, and harp on the few instances of deaths following vaccination which have been reported. A few bad results have occurred from improper technic and impure serum. This is not the fault of the method, it is due to the carelessness of the physician who vaccinates or of the manufacturers

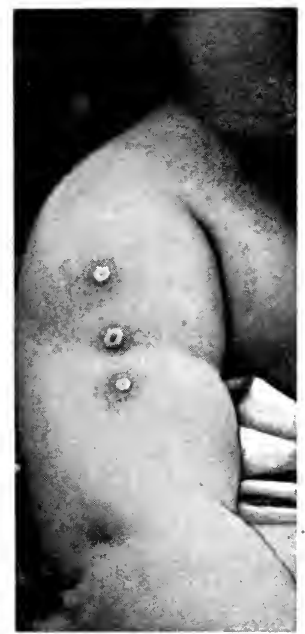

FiG. 88.- Normal vaccination pustule on the sixth day after vaccination (Frühwald and Westcott).

in making the serum. The risk at present is practically nil with modern methods of asepsis. In Prussia, where compulsory vaccination has existed since 1874 , the death rate per 100,000 of population has dropped from 24.45 per cent., the average previous to $\mathrm{I} 874$, to I.5I per cent.

A child, if healthy, should be vaccinated during the first two months of life, as the symptoms are very slight 
at that time. If delicate, wait until the child is in good condition and gaining in weight. A successful vaccination gives immunity for five years. Should the vaccination be unsuccessful, it should be repeated until a result is obtained.

Method.-The skin surface is washed clean and rubbed with alcohol. Do not use antiseptic solutions, as they kill the germ before it enters the system and a negative result follows. The upper layers of the skin are scraped off with a sharp bistoury until an exudation of serum takes place. Bleeding is to be avoided if possible. The serum is then introduced into the wound from the sterile tubes and thoroughly rubbed in by some flat instrument. The wound should be allowed to dry thoroughly before applying an aseptic dressing. This dressing should be renewed as long as there is an open wound and during the course of the local inflammation. It is as necessary to keep dirt and clothing out of this wound as any other. Severe ulcerations result from infection.

The course of the local manifestation is similar to that taken by the eruption of smallpox. First a papule, then a vesicle, umbilicated and loculated, followed by a pustule and a scab. It requires about the same time for development as the typical rash of variola. Three days is about the average for each stage.

\section{ERYSIPELAS}

An acute contagious disease excited by the streptococci and characterized by a peculiar inflammation of the skin and subcutaneous tissues. The germs can be carried in the clothes. Certain children are more predisposed than others. Those suffering from wounds or from diseases which lower the vitality are especially susceptible. 
The poison usually gains access through some wound or abrasion. In the newborn erysipelas starting at the navel is sometimes seen.

Symptoms.-The disease is ushered in with a chill and there is fever, $104^{\circ} \mathrm{F}$, , with all its symptoms. The inflammation usually begins in the neighborhood of the nose and spreads upward and laterally over the head to the neck, where it frequently stops. The affected part has a crimson hue, is swollen and tense, and is limited by a very sharp line or a well-defined ridge, beyond which, however, projections can be felt creeping out into the adjacent subcutaneous tissue. The surface of the inflamed area is first smooth and glazed, but later it is covered with minute vesicles and blebs. The child complains of burning and tingling and the surrounding skin may be so edematous that the features are almost unrecognizable. In four or five days the redness begins to fade and the swelling disappears; desquamation follows and the fever falls by crisis.

The average duration is from a week to ten days. Relapses are extremely common.

Nursing.-The dressings should be kept fresh, changing them at least twice a day. The patient shouid be kept quiet and the urine should be examined. The clothing and articles which come in contact with the patient must be disinfected. Never put a case of erysipelas in a surgical ward.

\section{PERTUSSIS OR WHOOPING-COUGH}

A contagious disease characterized by catarrh of the respiratory tract and peculiar paroxysms of cough, ending in a prolonged, whooping inspiration. The disease is 
highly contagious, proximity to the child with whoopingcough being sufficient to contract the disease. One attack protects from others.

Symptoms.-There are three stages, the catarrhal stage, the paroxysmal stage, and the stage of decline. Half of the cases appear in the first two years of life.

Catarrhal Stage.-The disease begins with the symptoms of coryza and bronchial catarrh. It is similar to other cases of bronchitis, but does not respond to the ordinary remedies for catarrh. After two or three weeks it passes into the paroxysmal stage.

In the paroxysmal stage the cough becomes more violent and is paroxysmal. During a paroxysm the face becomes cyanosed, the eyes are injected, and the veins distended. The cough frequently induces vomiting and in severe cases hemorrhages. The close of the paroxysm is marked by a long-drawn whooping inspiration due to the spasmodic closure of the glottis. The number of paroxysms varies; there may be from ten to twelve or, in severe forms, from forty to fifty in twenty-four hours. Ulcers frequently form on the frenum (the small attachment of the tongue to the floor of the mouth) from forcible propulsion of the tongue against the lower teeth during a paroxysm. This stage lasts about three or four weeks.

Stage of Decline.-The paroxysms gradually grow less frequent and less violent and finally cease.

Complications.-Pertussis is very prone to be followed by some complication. Bronchopneumonia probably ranks as the most frequent and fatal complication, causing two thirds of the deaths. Convulsions occur in some cases.

Prognosis.-During the first year the mortality is 
about twenty-five per cent. From this time on it rapidly decreases.

Treatment.-Children suffering from pertussis should be isolated so far as possible. They should be kept from school and any other children in the family sent away. Delicate children should be particularly protected against the disease. The isolated period should be continued as long as the spasmodic stage lasts.

Inhalations of menthol, amyl nitrite, or a few whiffs of chloroform will frequently control a violent paroxysm.

Nursing.- The child should have plenty of fresh air. During the day it is of benefit to take it out of doors. This is permissible if its temperature is not over $100^{\circ} \mathrm{F}$. At night the windows should be opened. Frequent changes of atmosphere and clothing seem to have a beneficial effect upon the spasms of cough. The seashore seems to aid in the cure of the disease.

If a child vomits a meal a short time after eating it, another meal should be given. It is best to make the diet as liquid as possible. Infants should receive their milk diluted more than usual, and in severe cases it should be peptonized (see page 339).

Light flannel underwear should be worn. The chest should be anointed with camphor oil daily, and inhalations of medicated steam should be employed.

A practical measure which may be adopted in those cases in which vomiting occurs frequently during the paroxysm is to place a binder around the abdomen. This should be drawn tight, as it is the support which it gives to the abdominal muscles which is desired. By preventing these large muscles from being brought into full play during the paroxysm vomiting is frequently 
avoided, as it is usually due to their violent contraction making pressure on the filled stomach.

Before disposing of the sputum and vomited material it should be disinfected.

\section{PAROTITIS OR MUMPS}

This is an acute contagious disease characterized by inflammation of the parotid and other salivary glands.

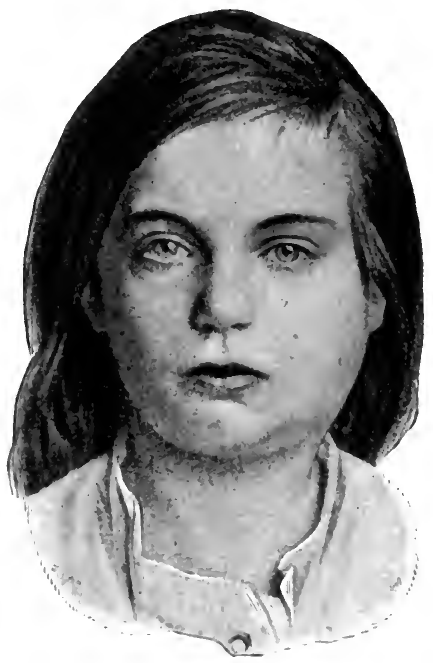

Fig. 89.-Epidemic parotitis. Second day. The picture shows the uniform swelling in the region of the left ear, which has spread to the face and the submaxillary areas; also the characteristic elevation of the auricular lobule. The filling of the fossa between the mastoid process and the ramus of the lower jaw is, unfortunately, not visible (Hecker, Trumpp, and $\mathrm{Abt}$ ).

The bacilli of the disease are probably contained in the sputa. One attack confers immunity.

Symptoms.-The disease is ushered in with chilliness, moderate fever, malaise, and a swelling of one of the 
parotid glands. The swelling is seen below and in front of the ear; the surrounding tissues are edematous and the submaxillary glands are soon involved. The features are swollen and distorted, the movements of the jaw are restricted and painful, and there is a decrease in the flow of saliva, making the mouth dry and uncomfortable. The other parotid is usually soon affected. The swelling lasts five or six days.

Complications are not often seen. Abscess of the parotid gland and deafness have been reported. Sometimes the testes in the male and, more rarely, the breasts or ovaries in the female are involved, but these complications are not common in childhood.

Treatment.-This consists of the applications of ichthyol ointment. The sputum should be disinfected.

\section{INFLUENZA OR LA GRIPPE}

An acute contagious disease characterized by fever, extreme prostration, pain in the head and back, and generally by catarrh of the respiratory or gastro-intestinal tract.

Etiology.-The disease occurs in epidemics. The exciting cause is a small bacillus found in the sputum. When prevalent no age or sex is exempt. One attack does not confer immunity from others.

Influenza does not kill except by its complications. The most frequent are catarrhal pneumonia, croupous pnuemonia, anemia, and otitis media. Tuberculosis of the lungs, nephritis, neuritis, and meningitis are also seen.

Symptoms.-The disease begins with languor, chilliness, severe pain in the head and neck, and fever ranging 
from $\mathrm{IOI}^{\circ}$ to $\mathrm{IO}^{\circ} \mathrm{F}$. There is extreme prostration. In some cases the principal symptoms are those of the respiratory tract, in others the gastro-intestinal symptoms are most important, and in the third variety the nervous symptoms are most prominent. In simple cases the temperature falls in two or three days by crisis, but complications not infrequently prolong the case for two or three weeks.

Respiratory Symptoms.-There may be coryza, laryngitis, or bronchitis. This gives rise to a nasal discharge, cough, expectoration, sneezing, and watering of the eyes. Tonsillitis and otitis media are often associated conditions.

Gastro-intestinal Symptoms.-There is vomiting and diarrhea with their attending symptoms.

Nervous Symptoms.-Neuralgic pains in the head, back, and limbs. In some children the nervous symptoms appear alone in conjunction with the fever.

One set of symptoms only may be present, but more often two or more tracts are involved. Skin eruptions occur at times.

Prognosis.-Uncomplicated cases nearly always recover in from five to seven days.

Treatment and Nursing.-The disease is communicable. The child should be put to bed and kept there until the fever is normal. A liquid diet should be given. The temperature, pulse, and respiration should be taken three times a day. If there is catarrh, the nose and throat should be systematically sprayed. Earache should be treated by syringing the ear with water at a temperature of $110^{\circ} \mathrm{F}$. The sputum should be disinfected. 


\section{CHAPTER XVI}

\section{CONSTITUTIONAL AND NUTRITIONAL DISEASES}

\section{Constitutional Diseases}

\section{RHEUMATISM}

RheumAtisM, as a whole, has varied manifestations in childhood. It may be articular, muscular, and neural. Rheumatic affections of the serous membranes, of the mucous membranes, and of the skin are found. The disease is uncommon under five years of age.

The two most prominent types of rheumatism are articular rheumatism and muscular rheumatism.

Acute articular rheumatism or inflammatory rheumatism is an acute general disease characterized by irregular fever, inflammation of joints, and a marked tendency to affect the heart. In children the acute course with marked inflammation of the joints is uncommon.

The cause of the disease is unknown. Some writers attribute acute articular rheumatism to bacterial infection, the germ being undiscovered. Poor hygienic surroundings, damp houses, and a sudden chilling of the body are the factors concerned in the onset of the disease.

Symptoms.-In children under ten years of age the disease begins slowly. There is fever ( $10^{\circ}$ to Ior $^{\circ} \mathrm{F}$.) and stiffness in several joints. Marked inflammation with swelling and pain of the joints involved is uncommon. 
The ankles, knees, wrists, elbows, and small joints of the feet are the ones most often affected. The stiffness may move from joint to joint or one alone may be involved. At times the joints are so stiff that they make the child lame; more often these pains are designated as "growing pains." Sweats are uncommon. The muscles are painful and there may be rigidity in more severe cases. The duration is from a few days to several weeks. One attack seems to predispose to others and anemia may result.

After ten years of age the symptoms closely resemble rheumatism in the adult.

Complications.-Endocarditis is the most common complication and may occur in very mild cases. About 40 per cent. of all cases of rheumatism have this complication. Pleurisy, pericarditis, pneumonia, chorea, iritis, meningitis, and certain cutaneous phenomena, such as purpura and urticaria, are also seen.

Pharyngitis, tonsillitis, laryngitis, and bronchitis are sometimes caused by rheumatism.

Muscular rheumatism is an affection of the voluntary muscles characterized by pain, tenderness, and rigidity.

Types.-Different names have been applied, according to the location.

Torticollis or wry-neck, when it involves the sternomastoid muscle.

Lumbago when it involves the lumbar muscles.

Pleurodynia when it involves the intercostals.

Cephalodynia when it involves the occipitofrontalis.

Exposure to the cold and wet, combined with muscular strain, usually excites it.

Symptoms.-Pain is the chief symptom, aggravated by 
the use of the muscles, and is associated with tenderness. Sometimes the muscles are rigid and contracted, such a condition being frequently seen in torticollis.

Torticollis. - The head is fixed and inclined to one side, every effort to turn it being accompanied with severe pain. The recovery is spontaneous in a few days.

Treatment.-Ironing the neck gives great relief. A small iron, not too hot, is the best implement to use. A piece of flannel should be laid upon the skin for protection.

Lumbago.-There is a dull, aching pain across the loins which is aggravated by turning the body or attempting to rise from a sitting posture.

Pleurodynia.-The pain is felt in the side and is increased by deep breathing, coughing, or twisting of the body. The respirations are restricted on the affected side and there is tenderness to the touch. The absence of fever will serve to distinguish it from pleurisy.

Cephalodynia is characterized by superficial head pains which are increased by movements of the scalp and are associated with tenderness on pressure.

Rheumatism frequently appears to be the cause of neuritis.

Treatment.-The salicylates and the alkalies are the remedies used in rheumatism.

Nursing.-The room should be kept warm and at an even temperature. On account of the danger of endocarditis the child should be kept in bed as long as there is fever. "Growing pains" should never be allowed to explain stiffness and discomfort in children. Involvement of the heart may follow such trivial symptoms, rheumatism not being suspected until a heart lesion is found. 
The child should be placed between blankets while in bed.

If applications to the joints are necessary, it must be done in a way to avoid pain.

\section{DIABETES MELLITUS}

This disease is characterized by the presence of sugar in the urine, polyuria, and loss of flesh and strength. The exact cause of diabetes is not known. It is uncommon in childhood, but when it exists the course is very rapid.

Symptoms.-Urinary.-The urine is increased in quantity, varying from three or four pints to six or eight quarts in twenty-four hours. It is light in color and of high specific gravity, I030 to I040, and leaves a whitish residue. In summer it attracts flies and has an aromatic odor. The total amount of sugar excreted in twenty-four hours can be from a few ounces to half a pound or more.

General.-The most prominent symptoms in childhood are loss of flesh and strength. The temperature is normal or subnormal and the thirst is unquenchable. The tongue and mouth are dry, the bowels are constipated, the skin is dry and harsh, and frequently the seat of intense itching. This is especially observed at the genitalia and may be the first symptom of the disease. There are also attending nervous symptoms, such as headache, disturbed sleep, enuresis, and abscess formations.

The course of diabetes mellitus in childhood is very rapid, much more so than in the adult, from two to four months being the average duration. The disease ends in a condition, analogous to uremia, called acetonemia, which is characterized by epigastric pain, dyspnea, a 
fruity odor to the breath, headache, delirium, stupor, and coma.

Nursing.-The room should be light and cheerful. Bathing should be frequent. Thirst should be satisfied. A specimen of urine should be saved daily unless otherwise ordered, and the amount voided in twenty-four hours measured. Symptoms of acetonemia must be reported immediately. The temperature, pulse, and respirations should be taken once a day.

Diet.-In diabetes the diet is of the utmost importance, all sugars and starches must be eliminated as far as possible.

Food-stuffs permissible in diabetes are: cream, curds, milk, eggs, buttermilk, fish, oysters, clams, all meats except liver, all green vegetables, unsweetened jellies, almonds, walnuts, butternuts, and pecans, tea and coffee with cream, but without sugar, and plenty of water. The bread should be made of gluten flour.

The food-stuffs that must be avoided are: all sugar, potatoes, white and sweet, rice, beets, carrots, turnips, peas, and beans. Pastry, unless made with gluten flour, and liver.

It is not necessary that the children should remain in bed, unless so ordered by the physician.

\section{HEMOPHILIA OR BLEEDER'S DISEASE}

This is an hereditary disease characterized by a tendency to bleed excessively from slight wounds or spontaneously. It is probably the worst condition with which a surgeon can be confronted. Children afflicted can bleed to death from a pin scratch, nothing apparently being of use to stop the hemorrhage. It usually runs in families, several 
brothers being affected; the sisters, however, are generally immune.

Symptoms.-The symptoms are free and persistent hemorrhage after a trivial injury. Spontaneous hemorrhages from mucous membranes of the nose, stomach, and bowel, and subcutaneous extravasations of blood are common.

Treatment consists in ligation, application of styptics, and plugging of the nose in epistaxis.

\section{PURPURA}

This condition is characterized by extravasations of blood into the skin. When there is likewise hemorrhage from the mucous membranes it is spoken of as purpura hemorrhagica.

Purpura occurs in quite a variety of conditions. It is seen in malignant cases of endocarditis, in severe cases of measles, scarlet fever, variola, and vaccinia, also in epidemic cerebrospinal meningitis and occasionally in diphtheria, rheumatism, pyemia, and septicemia.

Purpura also occurs late in the course of many of the protracted diseases, especially in infancy, such as bronchopneumonia, empyema, tuberculosis, enterocolitis, meningitis, and malignant cases.

Certain drugs, such as phosphorus, quinin, and potassium chlorate, may produce purpura when long continued.

Primary cases of purpura, not associated with any of the previously mentioned causes, occur in children of all ages, being not uncommon in infancy.

Symptoms.- The onset may be marked by some fever, headache, malaise, and pain in the limbs, but these may be absent and the disease ushered in by copious crops of 
small hemorrhages in the skin, followed by bleeding from the mucous membrane. Anemia and its associated phenomena develop in severe cases.

There may also be hemorrhages from the bowel, kidneys, and stomach.

It occurs at times in connection with rheumatism, when hemorrhages into the joints are seen, especially the kneeand ankle-joints. In the most severe forms gangrene of the mucous membranes involved is sometimes observed.

All forms of purpura have a tendency to relapse.

Prognosis.-The appearance of purpuric spots during the course of an infectious disease is always a bad sign, as it usually denotes a very severe infection. In the cachectic varieties it usually heralds the approach of death. Primary cases of purpura simplex generally recover. Primary cases of purpura hemorrhagica usually recover unless the fever is marked. The gangrenous form is fatal.

\section{NUTRITIONAL DISEASES}

\section{SCURVY, SCORBUTUS, OR BARLOW'S DISEASE}

This is characterized by bleeding, spongy gums, swelling and extravasations of blood around the joints, especially the knees and the ankles. There may be pseudoparalysis, immobility of the legs, extreme tenderness of the skin surface with swelling of the body and extremities, a thickening of the bones, marked anemia, and weakness.

Cause.-Prolonged use of condensed milk, sterilized milk, and proprietary foods.

The symptoms may come on slowly and last over several months. If the character of the disease is not recognized it may prove fatal. 
Treatment.-The disease yields readily to treatment. This consists in giving orange juice to the babies; in older children lemon juice is used.

\section{RACHITIS OR RICKETS}

This is a nutritional disease of early childhood, characterized chiefly by defective formation of the bony structures. It usually develops during the first or second year; it is not congenital. Poverty, artificial food, and bad hygienic surroundings are the predisposing causes. Breast-fed children rarely have rickets. The bones are soft, being extremely deficient in lime salts, and when ossification finally results the bones become heavy, large, and irregular in outlines. This causes such deformities as bow-legs, knock-knees, spinal curvature, pigeon-breast, and square cranium. The liver and spleen are often enlarged. The negro race is especially prone to the disease.

Symptoms.-The early symptoms are restlessness and slight fever at night, free perspiration about the head, diffuse soreness and tenderness of the body, prominence of the abdomen, pallor, slight diarrhea, delayed dentition, and the eruption of badly formed teeth.

Skeletal Phenomena.-The head is large and more or less square in outline; careful palpation may detect soft areas in the skull. The fontanel closes late. The sides of the chest are flattened, the sternum is prominent; nodules can be felt at the sternal end of the ribs called "rachitic rosary." There may be a distinct transverse groove at the level of the ensiform cartilage called "Harrison's groove." The spinal column is frequently curved anteroposteriorly called kyphosis; or latterly when it is 


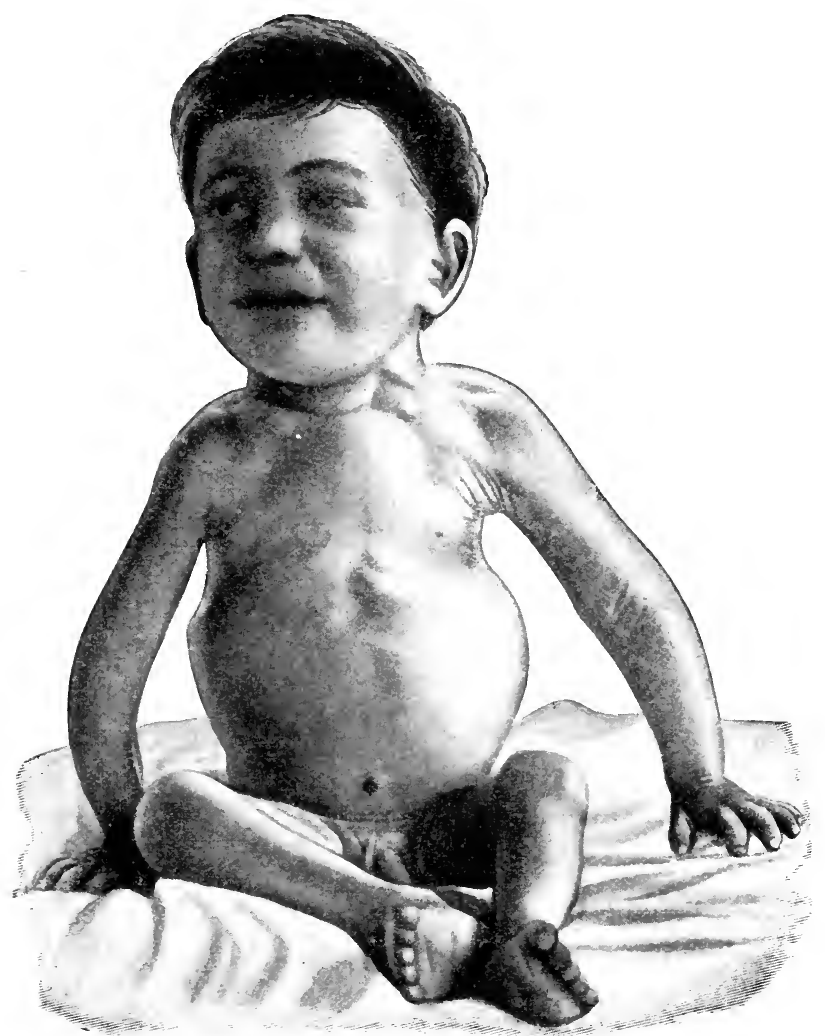

Fig. 90.- Rachitic boy of three years. A large and somewhat angular head. The typic posture of a rachitic child, with the arms supported at his side. Curvature of the clavicles and the spine causes the neck to appear short. Contraction of the lateral diameter of the thorax; abdomen protrudes; curvature of the bones of the forearm (Hecker, Trumpp, and Abt).

termed scoliosis. The long bones are curved and prominent at the extremities. This prominence leads to enlargements at the wrists and ankles.

The deformities of the legs may be marked. At times 
it is impossible for the child to stand erect and at others the whole skeleton is so deformed as to produce rachitic dwarfs. The usual deformities are knock-knees, bow-legs, and anterior bowing of the tibia. These are corrected by operative measures. The bones are broken, set straight, and kept in place by the use of plaster casts.

Prognosis.-Rickets in itself is rarely fatal. Some intercurrent disease, like pneumonia, may cause death.

Treatment.-This consists in giving the affected children the best milk obtainable and all the fresh air and sunshine they can get. Salt baths, prepared by placing three to five ounces of rocksalt in a gallon of water in the baby's tub, and rubbing the child until the skin glows, is advantageous.

Rachitic children should be taught to sit and to lie straight on account of the possibility of deformity. For the same reason they should not be permitted to walk until this danger has passed.

\section{MARASMUS OR INFANTILE ATROPHY}

When a child is unable to digest and assimilate food for any length of time a pathologic condition sets in. The symptoms arising from this are grouped under the names of malmutrition and marasmus. Marasmus is the extreme form of malnutrition, occurring so far as is known, without any constitutional or local disease. It is a result of faulty nutrition only.

The symptoms of marasmus are the same as those of malnutrition, only in a more advanced form. They are loss of weight until the child literally becomes skin and bones, pallor, ancmia, and subnormal temperature. The appetite in severe cases is almost entirely lost. The 
stools are sometimes normal, but more often contain curds and undigested food and are large in comparison to the amount of food taken. Bed-sores frequently develop and the children die of exhaustion.

Treatment.-This consists in discovering the food which is most easily assimilated and gradually increasing the strength of it until the normal percentages are properly digested. Such a diet may consist of egg-water, barley, arrowroot, and oat-meal water, malted soups, whey, buttermilk and milk mixtures, or, in a word, the entire subject of artificial feeding. Gavage may be necessary.

Nursing.-Nutritional diseases are due to a lack of proper food, consequently the careful preparation and administration of the proper diet is of the utmost importance. The character of the stools and vomit should be accurately reported and a careful chart of the child's weight kept. Bed-sores and pneumonia should be guarded against, and the temperature, pulse, and respiration taken at least twice a day. 


\section{CHAP'TER XVII}

\section{INFANT FEEDING}

NUTRITION is the most important branch of pediatrics. The question whether a child will be strong and robust or a weakling is often determined by its food in the first three months of its life. The corner-stone of the constitution is laid during that period. The largest part of the immense mortality of the first year is traced directly to disorders of nutrition.

At times temporary success may mean ultimate failure. This is illustrated in the use of many of the proprietary foods. The results seem to be satisfactory at first, the infant gains in weight remarkably, and the absence of certain vital elements from the food may not be noticed for months. It is finally discovered that the child has rachitis or some allied condition and it starts life handicapped by an undermined constitution. Another mistake often made is the prolonged use of predigested foods. The child is unable to digest naturally the simplest foods after prolonged use of such methods, this function being undeveloped. Some children with very robust constitutions seem to thrive on almost any food, but they are the exception.

\section{THE FOOD CONSTITUENTS AND THE PART THEY PLAY IN NUTRITION}

This is well describerl by Holt and in part his description is given below. "In infancy and childhood, as in 
adult life, the elements of the food are five in number: Proteids, fats, carbohydrates, mineral salts, and water. The forms in which they must be served to a child and the relative quantities in which they are demanded, are different from those required by an adult. One of the reasons for this difference is the delicate condition of the organs of digestion in infancy, and the inability to assimilate certain forms of food. Another reason is that provision must be made not only for the natural waste of the body, but for its rapid growth, as it nearly trebles in size in the first twelve months.

"Proteids.-These are essential to life since they are the only kind of food that is capable of replacing the continuous nitrogenous waste of the cells of the body, upon the healthy condition of which the digestion and assimilation of other elements of food depend. The proteid is furnished by the casein and other albuminoids found in both the woman's and the cows' milk. It is also found in muscle fiber, white of eggs, gluten of wheat, etc. The proteids most easily digested by infants are those of woman's milk. The greatest difficulty in artificial feeding has been to find other proteids to take their place. It is the difference in the digestibility of the proteids which causes most of the trouble in the substitution of cows' milk for woman's milk. The average amount of proteid furnished in a good sample of woman's milk is $\mathrm{I} .5$ per cent.

"Fats. - The uses of fats in the body are intimately associated with those of proteids. Fat possesses the important property of saving nitrogenous waste, so that when this is supplied in the food in proper proportions the entire energy of the proteid may be expended upon 
growth and nutrition of the cells of the body, without being used up in the production of animal heat. The demands upon the proteid by rapid growth of the body during infancy make it desirable that whenever possible the fats should do the work of the proteid.

"In-addition to their use as a source of animal heat the fats add to the body weight by storing up fat in the body. They are needed for the growth of the nerve cells and fibers and are essential to the proper growth of bone. Fat also fills the role of a natural laxative. The production of fat required in infancy is greater than at other periods of life. Probably the most common mistake in artificial feeding is to give too little fat. This is one of the chief reasons for the failure of proprietary infant foods, all being insufficient in fat. Woman's milk of good quality contains from three to five per cent. of fat.

"Carbohydrates.-Although these, like the fats, cannot replace the nitrogenous waste of the body, they are important aids to the proteids, and in this respect they are even more valuable than fats. The carbohydrates are partially changed into fat and may thus increase the body weight. They are capable of replacing the fat waste of the body, and are one of the most important sources of animal heat. The form in which carbohydrates are furnished to infants is milk sugar.

"In building up the cells of the body the proteids are first in importance, the carbohydrates second, and the fats third. In production of animal heat the necessity to maintain life, the fats come first and the carbohydrates second. The proteids should never be called upon for this purpose. In a proper diet all of the elements are represented. 
"Mineral salts are of greater importance in infancy than later in life, on account of the rapid building up of the bony system which is going on at this period. The most important for this purpose are the phosphate of lime and magnesium. These are furnished in abundance in both woman's milk and cows' milk. The salts are also necessary for cell growth, forming the mineral constituents of the blood and digestive fluids, and facilitating absorption, excretion, and secretion.

"Water.-The food of all young mammals consists of from 80 to 90 per cent. of water. This is needed for the solution of certain parts of the food, such as the sugars and some of the proteids and for the suspension of other proteids and emulsified fats. All the food is thus dissolved and very finely divided, so as to be more readily acted upon by the feeble digestive organs of the infant. Water is also needed in large quantities for the rapid elimination of waste in the body. When the diet of the infant is entirely fluid, additional water between feeding is unnecessary, but when solids are added and the feedings are at longer intervals, water should be given freely between feedings at all seasons, but more especially in summer."

\section{WOMAN'S MILK}

This is the ideal infant food. It is the secretion of the mammary gland. A few drops may be squeezed from the breasts before parturition. Generally speaking, however, it is only present after delivery. During the first two days the secretion is scanty, but usually upon the third or fourth day it becomes established. It is bluishwhite in color, usually alkaline, sometimes neutral, never acid in reaction. 'The specific gravity is I031, and when 
precipitated it forms light flocculi, never precipitating in large masses, like cows' milk.

Colostrum. - The secretion in the first two or three days differs quite markedly from the later milk. It is

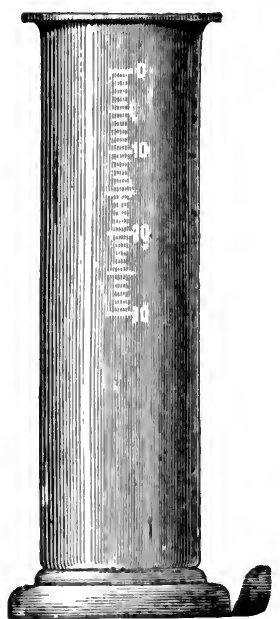

Fig. 91.-Crêmometer: The instrument is filled to the mark o with unskimmed milk and allowed to stand for from eighteen to twenty four hours at the temperature of the room, when the depth of the layer of yellow cream can be read off in degrees. The number of degrees corresponds to the cream percentage, which in good milk should never fall below 10 per cent. (after Chevalier).

of high specific gravity and very rich in proteids and mineral salts.

Composition of Woman's Milk.-Proteids are usually present in proportion of one to two per cent. The amount of proteid is larger in the first few days; after the third week it is stationary to the end of lactation, when it falls very markedly.

Fat : 4 per cent. is the average. 
Sugar: The ordinary variations are between 6 per cent. and 7 per cent.
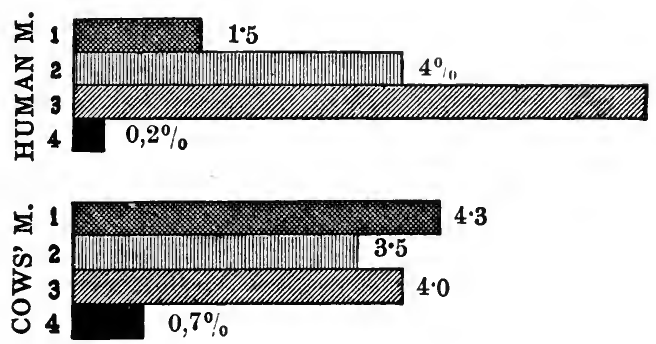

FiG. 92.-Scheme showing the composition of human and of cows' milk : I, Proteid; 2, fat; 3, carbohydrates; 4, salts (Frühwald and Westcott).

Salts: The average proportion is about 20 per cent. or about one-fourth that of cows' milk.

\section{EXAMINATION OF MILK}

The quantity is determined by weighing the baby before and after feeding. The average amount of milk taken at one feeding by a child is an ounce for each month of its age until the eighth month.

Reaction is obtained by means of litmus paper; specific gravity is obtained by means of a small hydrometer.

Fats.-A cream gauge holding ro cc. is filled to the zero mark, and allowed to stand for twenty-four hours at the temperature of the room. Then read off the percentage of cream. The ratio of cream to fat is, woman's 5 to 3. Cows', after eight to ten hours, 4 to I.

The sugar and salts are constant.

Proteids can only be approximately determined by the specific gravity and by the percentage of fats. A specific gravity higher than I03I shows increase in proteids; 
lower, a decrease, if percentage of fats is normal. Increased percentage of fats will lower the specific gravity, and a decreased percentage will raise it.

Microscopic examination shows the presence of bacteria, etc.

\section{COWS' MILK}

This is the only milk of lower animals practically available for infant feeding. It must be fresh, clean, from healthy animals, preferably of a mixed herd rather than from a single cow. It is then more apt to be uniform, as a single cow is subject to daily variations. Animals should have fresh food and transportation should be as short as possible. For all practical purposes it is necessary that one should know only the amount of fat in the milk being used, as this is the only variable factor.

The Difference between Cows' Milk and Woman's Milk.-Cows' milk is more opaque, slightly acid, or neutral, but never alkaline in reaction, as is woman's milk. There is less sugar in cows' milk, and the proteids in cows' milk are not only two or three times as great in amount, but they differ in their character. This latter is best shown by the digestibility of both proteids by the infant's stomach. Cows' milk in the stomach is coagulated into large, firm clots, which dissolve slowly, while woman's milk forms loose flocculent curds which dissolve readily.

Cows' milk contains bacteria; woman's milk is sterile.

Cream.-A great misapprehension exists as to the composition of cream. It is often spoken of as if it were entirely different from milk. It should be regarded as milk which contains an excess of fat, for the addition of cream to a mixture does not change the proteid percentage, but only the percentage of fat. 
In infant feeding it is convenient to make use of cream containing a definite percentage of fat. This may be either 8, I2, or 16 per cent. Sixteen per cent. is most often employed.

\section{METHODS EMPLOYED TO KILL THE BACTERIA IN MILK}

Pasteurized Milk.-The bottles, after having been thoroughly boiled, should be filled with the prescribed quantity of milk and corked with sterilized nonabsorbent cotton. Place the bottles in the cells of the pasteurizer and fill the cells with cold water. The cells are then

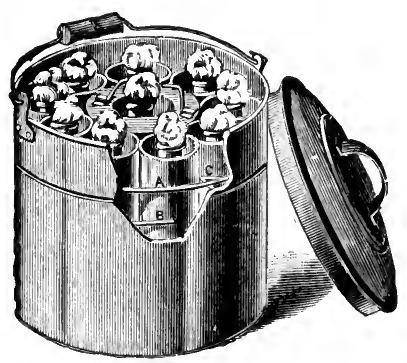

FIG. 93.-Freeman's pasteurizer.

placed in the pasteurizer, leaving out the middle set for the convenience of pouring in the boiling water. Next turn the faucet of the pasteurizer so that it will be open; then pour boiling water into the pasteurizer until it reaches the iron rods or until the water begins to run from the faucet; then turn off the faucet. Place in position the center cells and put the cover on securely. In a half hour remove the cover. Attach a hose to cold water faucet over the sink and another one to the faucet of the pasteurizer. Place the hose from the cold-water pipe inside the 
pasteurizer between the cells and the sides, turn on the cold water, and at the same time turn on the faucet of the pasteurizer, so that the water will run out at the bottom at the same time that it is running in at the top, thus causing a sudden cooling of the bottles, which is very important. After the bottles are thoroughly cold remove and place in the ice-chest. The pasteurizer should then be emptied by the hose attached to the faucet.

Three important facts must be remembered in pasteurizing milk. First, that the pasteurizer must stand on wood or on some other nonconductor of heat. Second, that the water poured into the pasteurizer must be boiling hot so that the temperature of the milk will be raised to $167^{\circ} \mathrm{F}$. in ten minutes. Third, that the bottles must be rapidly cooled.

To pasteurize milk when no regular apparatus is at hand, the bottles should be placed in a basket, preferably one made of wire, of which variety there are many on the market. A block of wood or a saucer should be placed in the bottom of a pan of cold water, and upon this stand the basket containing the bottles. This raises the bottles from actual contact with the bottom of the pan.

The water is then heated until the sterile thermometer placed in one of the bottles of milk reads $167^{\circ} \mathrm{F}$. This temperature should be maintained for one-half hour, when the bottles should be rapidly cooled under running water and placed on ice.

When sterilizing a thermometer by boiling, be sure to have one which will indicate $22^{\circ} \mathrm{F}$, otherwise it will break.

Sterilized Milk.-Prepare and fill bottles the same as for pasteurizing. The bottles are then set on a block of 
wood about one inch thick which is placed in a boiler so that the bottles do not touch the bottom. The water in the boiler should be then closely covered and allowed to steam for one hour. The bottles should then be removed, cooled, and put on ice.

\section{RECEIPTS FOR THE PREPARATION OF INFANT FOODS}

Barley Water.-Cover two tablespoonfuls of pearl barley with boiling water, let it boil five minutes, drain, and throw water away. Cover with two quarts of boiling water and simmer gently until reduced about one quart, which takes about two hours. Then strain through four thicknesses of gauze.

The prepared barley flour of the "Health Food Company of New York" or Robinson's Barley, two drams to each twelve ounces of water, and cooking for fifteen minutes is almost identical with the ordinary barley water (Holt).

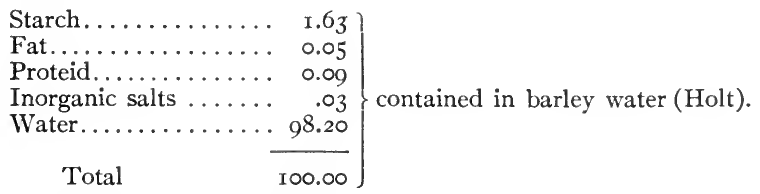

Rice and Oatmeal Water.-Cover two tablespoonfuls of rice or oatmeal with boiling water. Let it boil five minutes, drain, and throw water away. Cover with two quarts of boiling water and simmer gently until reduced about one quart. Then strain through four thicknesses of gauze. If used alone, add a little salt.

Rice Milk.-Wash a tablespoonful of the best rice and boil it one and a half hours in a pint of new milk. Rub it through a fine sieve. Add two tablespoonfuls of granulated sugar, heat, and serve. 
Albumin and Milk.-Put the white of one egg into eight ounces of cold milk. Pour the whole into a fruit jar, screw on the top and shake vigorously for half a minute. It is then ready to serve. It should be light and smooth.

Albumin Water.-Put the white of one egg into eight ounces of cold water. Pour the whole into a fruit jar and shake vigorously for half a minute, when it is ready to serve.

Whey.-Heat one pint of milk to $100^{\circ} \mathrm{F}$. (no higher), add one teaspoonful of rennet and stir gently, stand aside to set, but not on ice. After the milk has been thoroughly coagulated, stir up the curds with a fork or spoon, and strain through a flannel cloth. One quart of milk will make about two-thirds or three-fourths of a quart of whey.

In acute indigestion whey will often be retained when other foods are rejected.

Whey and White of Egg.-To every $7 \mathrm{oz}$. of whey add the beaten white of one egg.

Whey and Milk or Cream.-In adding milk, cream, or a milk mixture to whey, the whey should be made as directed above and after all the curds have strained off the whey should be heated to a temperature of $150^{\circ} \mathrm{F}$. and the milk, cream, or milk mixture must have been pasteurized before adding.

Whey, Milk, or Cream and White of Egg.-Add the quantity of pasteurized milk or cream at the temperature and in the manner stated above; then the mixture must be cooled or quite cold before adding the beaten whites of the eggs, the number of whites being one to every seven ounces of whey used, and not one to every seven ounces of the mixture. 
Wine Whey.-Heat a half pint of milk in a saucepan, and when steaming hot add, quickly, four tablespoonfuls of sherry wine. Let the mixture stand for a few minutes on the stove, then take from the fire, break up the curds, and strain through flannel or four thicknesses of gauze. It may be served with or without sugar.

Fully Peptonized Milk.-One pint of milk, 4 oz. of cold water, I peptonizing powder ( 5 gr. extract of pancreatis, I5 gr. soda bicarbonate).

Dissolve the powder in 4 oz. of water and to each bottle of milk of $4 \mathrm{oz}$. (previously pasteurized) add I oz. of the mixture. Stand the bottle in a pan of water $\mathrm{I} 20^{\circ} \mathrm{F}$. and allow it to remain there for a half hour, when it should be used at once. Peptonize each bottle to be used just before it is time for the feeding. Milk, fully peptonized, should only be given by gavage or by the rectum on account of its bitter taste. All milk ordered peptonized and given by gavage or rectum must be fully peptonized.

Partially Peptonized Milk.-One pint of milk, $4 \mathrm{oz}$. of cold water, I peptonizing powder ( 5 gr. extract of pancreas, ${ }_{5}$ gr. soda bicarbonate).

Dissolve the powder in 4 oz. of water and to each bottle of milk of $4 \mathrm{oz}$. (previously pasteurized) add I oz. of the mixture. Stand the bottle in a pan of water, $120^{\circ}$ F., and allow it to stand for ten minutes, when it is ready to be used at once.

Feedings of more or less than 4 oz. must be worked on the basis of I oz. of peptonizing solution to $4 \mathrm{oz}$. of milk.

Peptonized milk is valuable when there is feeble proteid digestion. It is not advisable to continue its use indefinitely, as the stomach gradually becomes less and less able 
to do its work. At the most it should be used only for a month or two at one time; when stoppage of this method is desirable, gradually diminish the amount of powder used.

Method of Peptonizing Mixtures.-First, make up mixture according to the formula. Then add the peptonizing fluid (made according to the formula) and allow the whole quantity of milk to stand in a bottle or pitcher in a pan of water, $120^{\circ} \mathrm{F}$., for the prescribed length of time, say ten minutes or a half hour, according to the order. Then quickly raise the water around the milk to the boiling-point and allow it to boil for three or four minutes. Remove the milk, cool, and bottle, and place on ice. The boiling of the water around the milk will stop the peptonization which, if not stopped, will cause the milk to become very bitter; it also sterilizes the milk and does away with the necessity of pasteurizing.

Oatmeal Gruel.-Mix two rounding tablespoonfuls of Bethlehem oatmeal with a little cold water; add a quarter teaspoonful of salt. Pour over it one pint of boiling water and stir over the fire until it boils. Then stand it where it will bubble slowly for a half hour, add a lump of sugar and a tablespoonful of whipped cream or a tablespoonful of sherry wine, and serve. Children seldom care for foods prepared with wine.

Apple Gruel.-Good in irritation of the bowels.

Core and quarter a large apple. Pour over it one pint of boiling water and simmer until it is reduced to a pulp. Strain. Mix two level tablespoonfuls of arrow-root with a little cold water and add to the hot apple water. Stir until it boils; then move back and let it cook slowly for ten minutes. Do not serve too hot and preferably without sugar. 
Farina Gruel.-Put a pint of milk into a double boiler. When it comes to the boiling-point sprinkle into it two level tablespoonfuls of Hecker's farina. Stir until it thickens and then let it cook for twenty minutes. Add a quarter of a teaspoonful of salt and a lump of sugar, and serve.

Flour Gruel or Pap.-Put a pint of milk into a double boiler and let it come to the boiling-point. Moisten two level tablespoonfuls of flour with a little cold water and stir into the boiling milk. Add one-fourth of a teaspoonful of salt and let it cook for twenty minutes. Add a lump of sugar and a little nutmeg if desired.

German Gruel made with Flour Ball.-Put one pint of flour into a strong bag and tie tightly with twine. Put into a kettle of boiling water and boil for five hours. When done take off the cloth and peel off the outside moist portion. Grate the ball and then put the flour into a baking pan and dry in a moderate oven for two hours, being careful not to brown.

Moisten two tablespoonfuls of this flour with a little cold water and pour over it one pint of boiling water and simmer for three minutes. Add a small pinch of salt and a lump of sugar and 4 oz. of warm milk, and serve.

Barley Gruel Liquefied or Dextrinized with Cereo or Maltine.-One heaping tablespoonful of barley flour, one pint of boiling water. Mix the flour in a small part of water and add to the rest. Boil fifteen minutes, then add enough cool water to make up the original pint. Cool to $100^{\circ} \mathrm{F}$. or $105^{\circ} \mathrm{F}$. and liquefy with one teaspoonful of cereo or maltine. This predigests the gruel.

Barley Gruel.-Moisten one tablespoonful of Robinson's Patent Barley with.4 oz. of cold water; pour over 
it $4 \mathrm{oz}$. of boiling water and add a half teaspoonful of salt. Let it simmer for five minutes; then add 4 oz. of hot milk. Let it come to a boil. Stir in a teaspoonful of sugar and serve.

Arrow-root Gruel.-Moisten an even tablespoonful and a half of arrow-root in a little cold water. Pour over it a pint of boiling milk, stir over the fire until it thickens and let it boil slowly for ten minutes. Take from the fire and add a teaspoonful of sugar and one-fourth teaspoonful of salt.

When this gruel is made for a child who is on a weak milk mixture, like 3.6.I. or weaker, the gruel should be made with two-thirds water and a third milk instead of a pint of full strength milk.

Arrow-root Gruel with Egg.-Separate an egg, beat the white and yoke until light; then mix them carefully. Add slowly one pint of plain freshly made arrow-root gruel, and serve.

Rice Flour Gruel.-Mix a tablespoonful of rice flour with a little cold milk and add it to a pint of scalding milk. Cook for fifteen minutes. Add one-fourth teaspoonful of salt, a teaspoonful of sugar, one-fourth teaspoonful of ground cinnamon, and a teaspoonful of brandy. This is especially beneficial as a food in cases of diarrhea.

Barley Jelly.-Put two tablespoonfuls of washed pearl barley into one and a half pints of water and slowly boil down to one pint. Strain and let the liquid settle into a jelly.

\section{Barley Jelly made with Robinson's Barley Flour.-} Dissolve slowly two rounding tablespoonfuls of Robinson's barley flour with two ounces of cold water. Add one pint of boiling water and simmer gently for fifteen minutes, 
stirring all the time. Strain and let the liquid settle into a jelly.

Beef Juice.-A piece of lean steak is slightly broiled on each side and the juice pressed out by a meat press or a lemon squeezer. Two or three ounces can ordinarily be obtained from one pound of beef. This is seasoned with salt and given cold or warm, but not heated sufficiently to coagulate the albumin in solution. If heated above $160^{\circ} \mathrm{F}$. it will be unfit for use.

Beef Juice and Milk.-When beef juice is added to milk the milk should never be heated above $100^{\circ} \mathrm{F}$. before the addition of the beef juice.

Barley Jelly, Maltine, and Milk Mixture.-Dissolve two teaspoonfuls of barley jelly (made with Robinson's flour) by adding one-fourth teaspoonful of Maltine and stirring. After the jelly has become a liquid add to the milk mixture in the proportion of the above quantity of liquid to every $4 \mathrm{oz}$. of mixture.

Preparation of Gelatin in the Treatment of Infantile Diarrhea.-Five hundred grams (- I7 oz., 3 dr. Io grs.) of chemically pure gelatin are dissolved in a liter $\left(-33^{\frac{1}{2}}\right.$ oz. $)$ of boiled water; the solution is filtered, and after being sterilized for an hour in an autoclave at a temperature of $248^{\circ} \mathrm{F}$, is poured into tubes having a capacity of Io cc. ( $-21 \frac{1}{2}$ fldr.), each tube thus containing I gm. (about I5 gr.) of gelatin. When it is desired to use this preparation, it is liquefied by placing the tubes in hot water. As much as $12 \mathrm{gm}$. or I4 gm. have been given in the course of twenty-four hours. Weill commences with $3 \mathrm{gm}$. a day and increases at the rate of I gm. a day until a decided effect is produced.

Oatmeal Jelly.-Soak two ounces of coarse oatmeal for 
twelve hours in one quart of cold water, then boil the mixture down to one pint, and strain while hot through a fine cloth or several thicknesses of gauze.

Malt Soup Mixture.-To make a 40 oz. mixture: $20 \mathrm{oz}$. of milk, $20 \mathrm{oz}$. of water, $3 \mathrm{oz}$., by measure, of wheat flour (measured loosely and not packed), $\mathrm{I}_{2} \frac{1}{\mathrm{oz}}$., by measure, of malt soup.

Number I. - Mix the flour with the $20 \mathrm{oz}$. of milk and suspend it so as to make a uniform mixture. After as much of the flour is dissolved or suspended as possible strain through gauze (two thicknesses) to strain out all the lumps or excess.

Number 2.-Dissolve the $\mathrm{I} \frac{1}{2} \mathrm{oz}$. of malt in $20 \mathrm{oz}$. of water.

Number 3.-Take the first mixture and the second mixture, that is, the flour and the milk mixture and the malt and the water mixture, and stir them together thoroughly. Place the whole in an enamel pot (or double boiler) and put over a slow fire, allowing the mixture to come to $160^{\circ} \mathrm{F}$. and keeping it at that temperature for twenty minutes, stirring all the time. At the end of the twenty minutes bring the mixture to a boil and remove from the fire. If there is a loss in the bulk through the cooking make up the full amount $(40 \mathrm{oz}$.) by adding sterile water; then place in the bottles and cool down slowly to the temperature of running water.

When the mixture is finished it has a light yellow color, smells of malt, and when it cools becomes quite thick, but when again heated becomes fluid and easily taken through the ordinary nipple if the holes are large.

When the malt soup mixture is ordered one-half or one-third strength, it means that instead of taking the full amount of milk (that is, the $20 \mathrm{oz}$.) half or only 
Io oz. of milk is to be used and the other half is to be water, making in the whole mixture, milk to oz., water $30 \mathrm{oz}$., instead of $20 \mathrm{oz}$. The extra ro oz. of water is to be added to the ro oz. of milk, and then the flour is dissolved or suspended in the half strength milk or proceed as directed above.

Buttermilk.-At times the proteid in plain cows' milk is indigestible even when given in very small percentages. Large curds continually appear in the stools. In such cases buttermilk often cures the indigestion in remarkably short periods of time.

It should be prepared as follows:

Flour

Sugar $3^{\frac{3}{4}} \mathrm{dr}$. by weight.

Buttermilk ................... I quart.

The mixture should be brought to the boiling-point, stirring continuously. Just as it is about to boil it should be removed from the stove and cooled rapidly under running water. The mixture should then be placed upon the ice. The proper amount for the age is poured into a nursing bottle at feeding time and slightly heated.

This mixture contains a much higher percentage of proteid than diluted cows' milk, but some change takes place in it which renders the mixture easily digestible. It should not be given for any length of time without the addition of cream.

Buttermilk Conserve.-This is a condensed form of buttermilk. One part is added to three or four parts of water. It is a very good method of serving buttermilk. It should be slightly heated before adding the water.

Buttermilk tablets are on the market under various trade names. If neither fresh buttermilk nor the conserve can be obtained, the tablets may be used. 
Condensed Milk.-It is made by heating milk to $212^{\circ} \mathrm{F}$. to destroy the bacteria and then evaporating in a vacuum at a low temperature to less than one-fourth its volume.

Condensed milk contains, after diluting six times, about I per cent. of fat, I.20 per cent. of proteid, 7.23 per cent. of sugar, and .I 7 per cent. of salts (Holt). As the usual dilution is from twelve to eighteen times it is evident how it lacks in fats and proteid. Knowing how necessary fats and proteids are to the infant it can be appreciated why condensed milk should not be used as a permanent food. It sometimes works well as a slight change for a short period in acute indigestion, but it should not be used permanently without the addition of cream, and never if good milk and accurate milk mixture can be obtained.

Junket.--To one pint of fresh luke-warm cows' milk add two teaspoonfuls of essence of pepsin or liquid rennet. Stir for a moment and then allow to stand until firmly coagulated. It is served cold.

Kumiss and Bean Flour.-These are preparations sometimes used in infant feeding. Kumiss is a fermented form of cows' milk. It is more useful for older children than for infants.

Bean flour has been recommended by Edsall for feedings in cases of difficult proteid digestion. Especially prepared flour must be obtained, and prepared according to the physician's instructions. 'The mixture has a nauseating odor, but it has given good results in a limited number of cases. 


\section{INFANT FOODS}

These are not in any way substitutes for mother's or properly modified cows' milk. They are capable of doing and have done much positive harm. They are the greatest exciting cause of rickets and scurvy. At times some of the preparations may be of considerable value, but chiefly for temporary use in pathologic conditions. Here they should be prescribed like drugs. The majority of the preparations are rich in sugar and lacking in fats and proteids. Children may gain weight, but they do so on the carbohydrates alone. The result of such development leads to the waxy appearance which children develop, when about a year of age, who have been fed on these foods. 


\section{CHAP'TER XVIII}

\section{ARTIFICIAL FEEDING}

THE various elements necessary for proper food in infancy, the difference between mother's milk and cows' milk, the various ways of preparing cows' milk to render it sterile or more easily digestible, and the various other preparations that may be used as substitutes for milk were discussed in the previous chapter, so that it is in order to discuss the subject of infant feeding proper.

The different methods of feeding which are available are breast-feeding, either by the mother or by a wetnurse; mixed feeding, or a combination of artificial feeding and nursing; and artificial feeding exclusively.

The first choice should always be maternal nursing. This is nature's food for the infant and nature cannot be improved upon.

"While recent advances in artificial feeding have greatly diminished the necessity for wet-nursing, there are still many instances where, objectionable though they may be, they are indispensable for saving the life of the child, as the perfect substitute for good breast-milk is as yet undiscovered" (Holt).

By mixed feeding is meant a combination of breast and artificial feeding. This may be resorted to when the milk supply of a mother is insufficient, or when the strain upon her health is unduly great. The same care must be 
exercised to keep the nipples clean and to have the feeding at regular intervals in breast feeding as in artificial diets.

Weaning should always be done gradually, when possible, for the sake of both mother and child. "While there are many women, especially of the lower classes, who are able to nurse their children advantageously throughout the first year, the number of such among the better classes is certainly very small. By the latter nursing can rarely be continued beyond the ninth, and often not beyond the sixth month, without unduly draining the vitality of the mother and at the same time harming the child. Weaning in hot weather is usually to be avoided.

"In cases of sudden weaning the food must be very much weaker in the beginning than for an artificially fed child of the same age. If weaned at six months the child should be put on a mixture suitable for a child of one month of age; if at nine or ten months, upon a food appropriate for a child of three or four months. If this is done the change can be made without causing much disturbance. When the infant has become somewhat accustomed to cows' milk, the strength can be gradually increased" (Holt).

\section{ARTIFICIAL FEEDING}

In artificial feeding there are several fundamental principles which must be constantly borne in mind; they are well described by Holt and his work is quoted, in part, below: "The food must contain the same constituents as mother's milk: namely, fat, proteid, sugar, inorganic salts, and water; the constituents must be present in about the same proportions as in good mother's milk; as nearly as possible the different constituents should 
resemble those of mother's milk both in their chemical composition and in their behavior in the digestive fluids; the addition to foods of very young infants of substances not found in mother's milk, like starch, is unnecessary, contrary to the best physiology, and if used in considerable quantities may be positively harmful.

"In the artificial feeding of infants, cows' milk is selected because it furnishes all the necessary elements, although not in proportions required by young infants. In feeding infants according to this plan the attempt is made so to modify cows' milk as to make it conform in composition to woman's milk, and so to adjust the proportions of the various constituents to meet the individual cases.

"In modifying cows' milk for infant feeding our calculations are based upon the composition of good breast milk, as determined by the latest analyses:

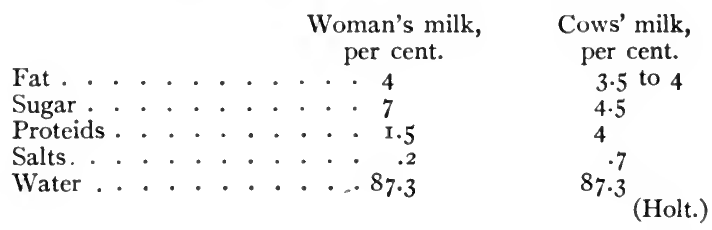

"In cows' milk there is an excess of proteids and salts, too little sugar, and about the quantity of fat required. Other conditions which must be considered are the presence of bacteria in cows' milk, its acid reaction, and the fact that its proteids are more difficult of digestion.

"Fats.-The average amount of fat that an infant can digest varies from 2 to 4 per cent. It is rarely necessary in health to go above or below these proportions. Beginning with the 2 per cent. in the early days of life, this can be increased to 3 per cent. in a month, and to 4 per 
cent. at the age of five or six months. No other modification in the fat is necessary.

"Sugar.-In woman's milk the percentage of sugar is constant in all instances, between 6 and 7 per cent. In feeding cows' milk it is seldom necessary to have the sugar less than 5 per cent. and never more than 7 per cent. It should be distinctly understood that the purpose of adding sugar to milk is not to sweeten it, but to furnish the proper proportion of soluble carbohydrate necessary for the infant's nutrition. However, when good milk sugar cannot be obtained, cane sugar may be used. The amount added must be but little more than half that of milk sugar on account of its sweeter taste and its greater liability to ferment in the stomach.

"Proteids.-The modification of the proteids is the most important change necessary in cows' milk, for it is the proteids which give the greatest difficulty in infant digestion. In ordinary cases in health, a reduction in the amount of proteids present is all that is necessary. The normal amount of proteids in woman's milk is $\mathrm{I} .5$ per cent. In very young infants it is necessary to reduce it even more than this, sometimes to .75 per cent. and even to .50 per cent. By the end of the first month the average child can take I per cent. and by the fourth month I.5 per cent. and by the sixth month 2 per cent. The reduction of the proteids is effected by dilution with water."

The meaning of such terms as 3.6.r. mixtures etc., is 3 per cent. fat, 6 per cent. sugar and I per cent. proteid mixture. Mixtures should always be expressed in the sequence of fat, sugar, proteid.

"Inorganic Salts.-These, like the proteids, are exces- 
sive in cows' milk and to nearly the same degree. Therefore, when milk is diluted as required by the proteids, the salts will be nearly in their proper proportion and they may be dismissed from separate consideration.

" Reaction.-The acidity of cows' milk may be overcome by the addition of either lime-water or bicarbonate of soda. Of the former, 5 per cent. of the total quantity is required; of the latter, one grain to each ounce of food."

The subject of heating milk for the destruction of bacteria was considered on page 335 .

Fat is furnished by cream; proteid by milk. Cream containing 16 per cent. fat is usually taken as a working basis, and a standard solution of milk-sugar of 20 per cent. strength should be used.

\section{CREAM}

In the larger cities it is possible to obtain certified half-pints of cream of 16 and 12 per cent. strengths.

When these are not available and the nurse has to depend upon an ordinary quart bottle of milk to obtain the cream, she will find the following table of service.

After a quart of milk, containing 4 per cent. fat, has stood for at least twelve hours,

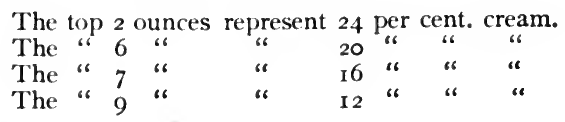

If a pint of milk is used, one-half of the above quantities represent the percentages of fat; for example, $3^{\frac{1}{2}}$ ounces would equal a 16 per cent. cream, and $4 \frac{1}{2}$ ounces a 12 per cent. cream.

Milk containing 4 per cent. fat is about the average 
quality served to the public. It is not the richest milk obtainable, as this is not desired. The cream line of certified 4 per cent. milk in the ordinary shaped quart milk bottle is about 4 inches below the top, and when the visible cream is dipped off it will amount to about 5 ounces.

Four per cent. fat milk means that if the quart should be shaken until an even distribution of the cream is obtained there would be 4 per cent. of fat in every ounce of milk in the bottle, whether the first or the thirty-second is used.

When milk stands the fat rises to the top of the bottle and only the upper ounces contain it; if these should be dipped off, the bottom ounces would be entirely free from fat ("skimmed milk").

-It must also be remembered that in obtaining definite strength creams, it is not meant that the top 7 or 9 ounces should be entirely composed of cream. Both the cream and the milk below the cream line must be used.

This is obtained by two methods: (I) by siphoning, (2) by dipping.

Method for Siphoning.-A sterile glass tube long enough to reach to the bottom of a quart jar and curved at its upper extremity is placed in the bottle of milk. To its outer end a sterile rubber tube is attached, which runs to the sterile receptacle which is to receive the bottom milk. The bottom of the bottle of milk must be on a higher level than the receptacle. To start the flow of milk introduce a large $\frac{1}{2}$-ounce sterile eye-dropper into the free end of the rubber tube. The bulb of the dropper must be tightly squeezed between the fingers when this is done. As soon as the dropper is in position release the bulb and the suction will 
draw the milk up the glass tube. Withdraw the dropper as soon as the milk has turned the curve of the glass tube. If, for any reason, the first attempt at suction is not successful, do not squeeze the bulb again without withdrawing the dropper, for if the bulb should be squeezed while still in position it would disturb the cream layer.

Do not start the flow under any circumstances by sucking with the mouth over the free end of the tube. The mouth is not sterile and would contaminate the end of the

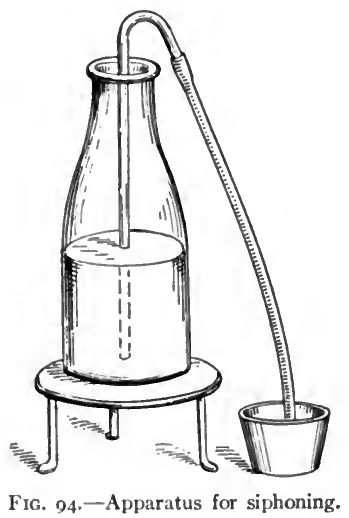

tube through which the milk flows. The milk is allowed to flow until all of the bottom milk is removed from the bottle and only the desired amount of top milk remains.

For instance, to get a 16 per cent. cream, 25 ounces of the bottom milk would be siphoned off, leaving 7 ounces of 16 per cent. cream.

Method for Dipping.-A special dipper is necessary, the best being a Chapin dipper.

The first ounce of cream in the neck of the bottle must be removed by a teaspoon. Then the dipper is introduced 
in such a way that its upper edge is absolutely on a line with the top of the cream. The dipper is gradually lowered as the cream runs into it. The dipper contains I ounce, and the amount of top milk desired is removed by successive dipperfuls.

Method for Changing Percentages of Cream.-To make I2 per cent. cream, take two parts of 16 per cent. cream

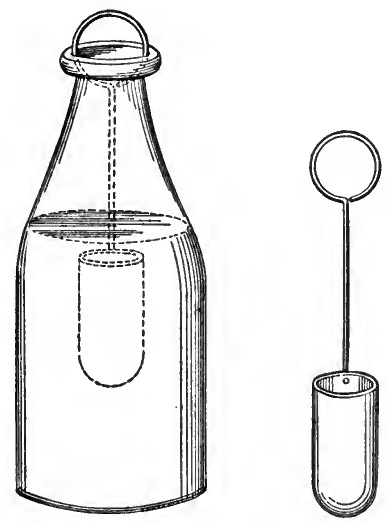

FIG. 95.-The Chapin dipper.

and one part of whole milk. Example: To make 9 dr. of I 2 per cent. cream, take $6 \mathrm{dr}$. of $\mathrm{I} 6$ per cent. cream and 3 dr. of whole milk.

Eight per cent. cream contains one part i6 per cent. cream and two parts of whole milk.

Example: To make 8 per cent. cream, take 2 oz. of I6 per cent. cream and add 4 oz. of whole milk to make $6 \mathrm{oz}$.

\begin{tabular}{|c|c|c|}
\hline r6 per cent. cream....... I6 & $\begin{array}{l}\text { Sugars. } \\
4\end{array}$ & $\begin{array}{l}\text { Proteids. } \\
3.60\end{array}$ \\
\hline I 2 per cent. cream....... I 2 & 4.20 & 3.80 \\
\hline 8 per cent. cream....... 8 & 4.20 & 3.90 \\
\hline
\end{tabular}


The fats alone are increased in cream, the sugar and proteid remain practically the same. Cream is the same as milk, with the addition of all the fat in the bottle which has floated to the surface.

\section{METHODS BY WHICH MILK CAN BE MODIFIED}

There are several formulæ to expedite this work. At the Children's Hospital in Philadelphia the nurses construct the milk mixtures from the following:

\section{Baner's Formula.-}

$\mathrm{Q}=$ Total quantity to be used in twenty-four hours.

$\mathrm{F}=$ The per cent. of fat desired in the mixture.

$\mathrm{S}=$ The per cent. of sugar desired in the mixture.

$\mathrm{P}=$ The per cent. of proteid desired in the mixture.

$\mathbf{M}=$ milk. $\mathbf{C}=$ cream. $\mathrm{L}$. $\mathbf{W}$. = lime-water.

$\frac{\mathrm{Q} \times(\mathrm{F}-\mathrm{P})}{\text { percentage of . cream }-4}=$ cream.

$\frac{\mathrm{Q} \times \mathrm{P}}{4}$ - the cream $=$ milk.

5 per cent. of the total quantity =lime-water.

$\mathrm{Q}-\mathrm{M}-\mathrm{C}-\mathrm{L}$. W. = sterile water.

$\frac{\mathrm{Q} \times(\mathrm{S}-\mathrm{P})}{100}=$ milk sugar.

Example.-A 4.7.2. mixture is ordered, $40 \mathrm{oz}$. to be given in twenty-four hours. The percentage of the cream used is $I 6$.

$$
\begin{aligned}
& \mathrm{Q}=40 . \quad \mathrm{F}=4 . \quad \mathrm{S}=7 . \quad \mathrm{P}=2 . \\
& \frac{40 \times(4-2)}{\mathrm{I} 6-4}=\frac{40 \times 2}{\mathrm{I} 2}=6 \frac{2}{3} \text { oz. cream. } \\
& \frac{40 \times 2}{4}-6 \frac{2}{3}=\mathrm{I} 3 \frac{1}{3} \text { oz. milk. } \\
& \mathrm{I} \frac{\mathrm{I}^{5} 0}{\mathrm{o}} \text { of } 40=2 \text { oz. lime-water. } \\
& 40-6 \frac{2}{3}-13 \frac{1}{3}-2=18 \text { oz. of sterile water. } \\
& \frac{40 \times(7-2)}{100}=\frac{40 \times 5}{100}=2 \text { oz. milk sugar. }
\end{aligned}
$$

The proper proportions for a $4^{-7-2}$ mixture, $40 \mathrm{oz}$. to be given in twenty-four hours, based upon 16 per cent. cream are: 


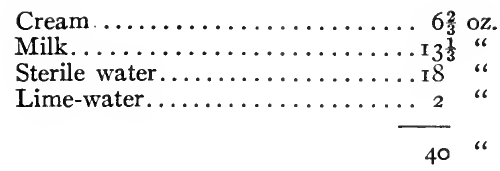

Two ounces of dry milk sugar are dissolved in this mixture.

Short Cuts.-In a 3.6.I. mixture the cream equals one-sixth of the total quantity, if I6 per cent. cream is used; and the milk equals one-fourth of the total quantity.

In a 3.6.I. mixture if a 12 per cent. cream, both the fat and the proteid are furnished by the cream and the addition of milk is unnecessary.

Example.-Total quantity 32 oz., a 3.6.I. mixture made with I 2 per cent. cream.

$$
\begin{aligned}
& \frac{32 \times(3-I)}{I 2-4}=\frac{32 \times 2}{8}=8 \text { oz. cream. } \\
& \frac{32 \times 1}{4}-8=0=\text { milk. }
\end{aligned}
$$

In a mixture where citrate of soda is used the powder is added in the proportion of I gr. to each ounce of milk or cream. The 5 per cent. of lime-water is not used in such a mixture, the citrate of soda giving the necessary alkalinity.

In using a solution where 2 gr. $=\mathrm{I}$ dr., or any other solution, the amount of water used to dissolve the necessary number of grains should be subtracted from the total quantity of sterile water.

Whenever milk is mentioned, it means whole milk, that is, milk that has not been skimmed, and in which a thorough distribution of the cream has been obtained by shaking the bottle.

In diluting the whole milk to reduce the proteid, the fats 
are equally reduced. Therefore, it is necessary to add an extra amount of fat to the mixture to bring up its percentage to the proper strength, which is higher than the percentage of proteid desired; for this reason the cream is always added to whole milk.

\section{TOP-MILK MIXTURES}

In private practice it is often more convenient and less expensive to use top-milk mixtures instead of adding separate standard cream (like 16 per cent. cream) to a quantity of whole milk, in order to increase the amount of fat in a mixture of cows' milk.

Cows' milk contains, for all practical purposes, 4 per cent. of fat, 4 per cent. of sugar, and 4 per cent. of proteid. If I per cent. of proteid is desired, it is readily obtained by diluting the whole milk with three times its volume of water, this makes the milk one-quarter of its original strength or I per cent. proteid. If 2 per cent. proteid is desired, the whole milk is diluted with an equal quantity of sterile water. This makes the proteid one-half its original strength or 2 per cent. The fats and sugars are necessarily reduced in the same ratio.

As was said before, the top 7 ounces of a quart of milk contain a 16 per cent. cream after standing twelve hours, and the top 9 ounces a i 2 per cent. cream. The fats alone are increased in cream. The proteids are still approximately 4 per cent.

If a $3^{-6-1}$ mixture is ordered by the physician, and the child is taking ten bottles of $2 \mathrm{oz}$. each during the twentyfour hours, the total quantity for the day would be $20 \mathrm{oz}$. Therefore, it is necessary to make $20 \mathrm{oz}$. of a mixture containing 3 per cent. fat, 6 per cent. sugar, and r per cent. proteid. 
Method.-The top $9 \mathrm{oz}$. should be dipped, or the lower 23 oz. siphoned, off. This must be carefully done to avoid the risk of shaking up the cream and not obtaining the full strength of fat. This gives $9 \mathrm{oz}$. of milk containing I2 per cent. fat, 4 per cent. sugar, and 4 per cent. proteid.

If this quantity should be diluted with three times its volume of water it would represent 3 per cent. fat, I per cent. sugar, and I per cent. proteid, as the milk would be only one-fourth of the whole mixture.

If a I2 per cent. cream is used, and 3 per cent. fat is desired, the total quantity should be divided by onefourth. Therefore, $\frac{1}{4}$ of $20=5$. Hence, $5 \mathrm{oz}$. of the original 9 ounces of the 12 per cent. cream is the proper amount in this example. To this must be added the proper amount of lime-water and sugar of milk, which may be worked out by Baner's formula, and enough sterile water to make the mixture the proper total quantity. Example:

$$
\begin{aligned}
& \frac{1}{4} \text { of } 20=5 \text { ounces of } 12 \text { per cent. cream. } \\
& I^{5} \text { of } 20=\mathrm{I} \text { ounce of lime-water. } \\
& \frac{20 \times(6-1)}{100} \times \frac{20 X 5}{100}=\mathrm{I} \text { ounce of sugar of milk, which dis- }
\end{aligned}
$$

solves and does not increase the quantity of the mixture.

Subtracting 6 oz. (top milk and lime-water) from 20 equals I4 oz. of sterile water necessary to complete the mixture. Thus:

Top milk........... 5 ounces

Lime-water............ I ounce

Sterile water........... I4

Sugar of milk........... I ounce, which dissolves. 
The 5 oz. represent $\frac{1}{4}$ of 20 , the dilution being 15 oz., so the fat is 3 per cent. and the proteid is I per cent. The sugar, which was reduced to I per cent. by this dilution, is raised to 6 per cent. by the addition of the ounce of sugar of milk.

That the top $9 \mathrm{oz}$. contain practically all the fat in the quart of milk can be proved by multiplying 9 by the percentage of fat and dividing by 100: $\frac{9, \times_{12}}{100}=1.08$ ounces of fat in 9 ounces, and then subtracting this amount from the total quantity of fat in $32 \mathrm{oz}$. of a 4 per cent. milk, $\frac{32 \times 4}{100}=1.28$ ounces of fat in a quart, which subtraction leaves .20 oz. of fat in the remaining $23 \mathrm{oz}$. of bottom milk, which is practically skimmed milk, and the addition of any amount of these bottom $23 \mathrm{oz}$. to a mixture would not raise the percentage of fat, but only the proteid. Therefore, if a 3-6-2 mixture is desired, it is only necessary to add some of the bottom milk to bring up the percentage of the proteid.

Thus, $20 \mathrm{oz}$ of a $3^{-6-2}$ mixture are desired.

From the previous example we know the quantities necessary to make a $3^{-6-1}$ mixture.

By dividing the total quantity by $\frac{1}{4}$ we get the proper dilution of the fat to 3 per cent., but the proteid is likewise reduced to I per cent. Here it is necessary to raise the proteid to 2 per cent. This can be done as follows:

If whole milk contains 4 per cent. of proteid, diluting with an equal amount of sterile water would give a 2 per cent. proteid, but we have already diluted with three parts of water, reducing the proteid to I per cent., therefore, by adding an amount of skimmed milk equal to the quantity 
of top milk used, we will double the percentage of proteid without disturbing the fat, consequently the result would be:

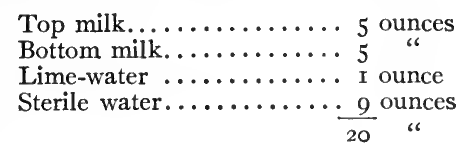

Sugar of milk.......... I ounce, which dissolves.

One-half the mixture is milk and cream and one-half water.

In this same manner any percentage of proteid can be worked out if it is remembered that one-quarter of the total quantity represents I per cent. of proteid.

Thus, if $\mathrm{I} \frac{1}{2}$ per cent. of proteid is ordered in a $20-0 z$. mixture, and $5 \mathrm{oz}$. represent I per cent., it would be necessary to add $2 \frac{1}{2} \mathrm{oz}$. of bottom milk.

As it is only possible to obtain $9 \mathrm{oz}$. of 12 per cent. cream from I quart of milk, anything above $36 \mathrm{oz}$. of total mixture will require the purchase of an extra pint or quart of milk.

For fat percentages lower than 3 per cent., it would be necessary to take smaller fractions; thus, one-sixth of the total quantity would represent a 2 per cent. fat if a I 2 per cent. cream is used. The proteid would be reduced to two-thirds of I per cent., and sufficient bottom milk would have to be added to bring up the percentage.

Example.-If $24 \mathrm{oz}$. of a $2-6-\mathrm{I}$ mixture is ordered:

One-sixth of $24 \mathrm{oz}$. equals $4 \mathrm{oz}$. of top I 2 per cent. milk, representing 2 per cent. of fat and two-thirds of I per cent. of proteid.

If two-thirds of I per cent. is contained in 4 oz., I per cent. will be represented in $6 \mathrm{oz}$.

$$
\frac{2}{3}: \mathrm{r}:: 4: \mathrm{x}=4 \div \frac{2}{3}=6 \text { ounces. }
$$


Therefore, 2 oz. of bottom milk must be added. The formula would then read:

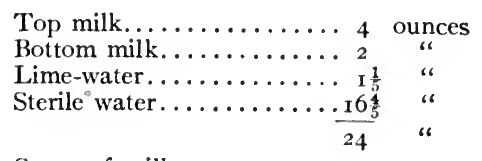

Sugar of milk......... Is ounces, which dissolves.

The quantity of lime-water and sugar of milk are determined by Baner's formula.

Another method which can be used to arrive at the same result is to work with two bottles of milk. If the amount to be used is small, two pint bottles will answer.

The top $3 \frac{1}{2} \mathrm{oz}$. of milk in a pint represent a 16 per cent. cream. This amount can be dipped off and the quantity of I6 per cent. cream found necessary by Baner's formula can be added to the amount of whole milk required. The whole milk is obtained from the second bottle, which has been thoroughly shaken to get a uniform distribution of the cream. A I 2 per cent. cream can be obtained by using $4 \frac{1}{2} \mathrm{oz}$. Two quart bottles can also be used when the quantities required cannot be furnished by the pints.

Example.-Forty ounces of a 4-7-2 mixture are desired:

The top 7 oz. of a quart give a 16 per cent. cream. Working with Baner's formula we find that $6 \frac{2}{3} \mathrm{oz}$. of a 16 per cent. cream are necessary; therefore this amount is taken from the top $7 \mathrm{oz}$. and added to $13 \frac{1}{3} \mathrm{oz}$. of whole milk taken from the second bottle, which amount is found to be the proper quantity to add to this mixture by working the formula (see page 356 ). 


\section{METHODS FOR DETERMINING THE PERCENTAGES OF VARIOUS MIXTURES}

Whole cows' milk contains 4 per cent. of fat, 4.5 per cent. of sugar, and 4 per cent. of proteid.

Milk and Lime-water, 5 to I.-

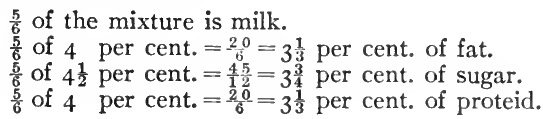

Therefore, this mixture contains $3 \frac{1}{3}$ per cent. of fat, $3 \frac{3}{4}$ per cent. of sugar, and $3 \frac{1}{3}$ per cent. of proteid.

Milk and Lime-water, 3 to I.-The percentages are determined in the same way, three-fourths of the whole quantity being milk.

Whey, 5 oz. +1 oz. of a 16 Per Cent. Cream.Whey contains 0.32 per cent. of fat, 4.79 per cent. of sugar, and 0.86 per cent. of proteid. Cream contains I6 per cent. of fat, 4 per cent. of sugar, and 3.6 per cent. of proteid.

In this mixture one-sixth of the total quantity is cream.

$\frac{1}{6}$ of $\mathrm{r} 6$ per cent. $=\frac{16}{6}=2.66$ per cent. of fat contained in cream. $\frac{5}{6}$ of $.3^{2}$ per cent. $=\frac{1.60}{6}=.26$ per cent. of fat contained in whey.

$2.9^{2}$ per cent. of fat contained in mixture.

$\frac{1}{6}$ of 4 per cent. $=\frac{4}{6}=0.66$ per cent. of sugar contained in cream. $\frac{5}{6}$ of 4.79 per cent. $=\frac{23.95}{6}=3.99$ per cent. of sugar contained in whey. 4.65 per cent. of sugar contained in mixture.

$\frac{1}{6}$ of 3.60 per cent. $=\underset{6}{3.60}=.60$ per cent. of proteid contained in cream. $\frac{5}{6}$ of .86 per cent. $=\frac{4.30}{6}=.7 \mathrm{I}$ per cent. of proteid contained in whey. I. I $^{\mathrm{I}}$ per cent. of proteid contained in mixture.

The mixture contains 2.92 per cent. of fat, 4.65 per cent. of sugar, and I.3 I per cent. of proteid.

Rule for Determining the Percentage of Fat in a 
Mixture.-Add the quantities of the ingredients together. Multiply the percentage of the fat in the cream by the quantity of cream in the mixture and divide by the total quantity. Multiply the percentage of fat in the milk by the quantity of milk in the mixture and divide by the total quantity. The sum of the two results gives the percentage of fat in the mixture.

Example.-In a mixture containing 5 oz. of a 16 per cent. cream, II oz. of milk (4 per cent. fat) and $24 \mathrm{oz}$. of water, the total quantity is $40 \mathrm{oz}$.

$$
\begin{aligned}
& \frac{I 6 \times 5}{40}=\frac{80}{4}=2 \text { per cent. of fat in the cream. } \\
& \frac{4 \times I I}{40}=\frac{4}{40}=\frac{\text { I.I per cent. of fat in the milk. }}{\text { 3.I per cent. of fat in the mixture. }}
\end{aligned}
$$

Therefore, the mixture would contain 3.I per cent. of fat.

\section{CARE OF MILK IN THE HOUSE}

The best milk may be absolutely spoiled by carelessness in the methods employed for keeping it in the house. Too often in the large cities the bottles are left at the front or back door by the distributor at a very early hour in the morning. Two or three hours often pass before the milk is placed on ice; this may be during the hottest days of summer, and often after it has stood in the direct rays of the sun. Necessarily the milk should be placed immediately in the refrigerator. Some very ingenious devices are constructed by many to receive the milk and save the early morning rising, at the same time having the milk in proper surroundings. If the milk is not delivered in hermetically sealed bottles then nothing is better to keep it in than an ordinary mason jar which has been properly 
sterilized. The milk should not be allowed to stand in the refrigerator uncovered; nor should it be placed in the same compartment with the food. The best method to employ, if a separate compartment is not available, is to have a small refrigerator for the milk alone; many of these are on the market. However, a very satisfactory one can be improvised from an ordinary bread-box. The refrigerators must be kept scrupulously clean. If at any time there is a disagreeable odor perceptible upon opening the box, it is either due to neglect or to a leak into the packing between the walls of the refrigerator. As this packing is often hair, wool, or some similar substance, the water renders it mouldy and consequently unhealthy; when such a contingency occurs the refrigerator should be immediately abandoned. After the milk is on the ice it should be disturbed as little as possible, hence it is better to prepare the milk for the day at one time. Each feeding should be placed in a separate nursing bottle properly sterilized and stoppered with aseptic cotton. These bottles are placed in the refrigerator immediately. It is better to have the ice in a separate compartment from the milk, as the water which collects fom the melting of the ice is not pure, often containing dirt, and if from artificial ice, traces of ammonia. If, for any reason at all, a nursing bottle should topple over into this water, the milk within it may become contaminated. It is well to leave the bottles stand in a wire frame.

Milk left uncovered for fifteen minutes may render all the care and aseptic measures practised at the dairies useless.

The same care must be used in handling condensed milk and buttermilk. All can openers used to open tins 
containing the commercial varieties of these products must first be boiled. The entire contents of the can must be emptied into a sterile nursing bottle or some similar receptacle and kept on ice. Precautions must be taken to prevent contamination by placing sterile cotton in the mouth of the bottle and covering other forms of receptacles thoroughly. If a can of condensed milk is slightly warmed, by placing it in hot water for five minutes, the contents will run easily into a nursing bottle. Condensed milk that has been open for more than two days should not be used.

Be careful to keep the cotton stoppers sterile while filling the bottles; do not carelessly place them where they may be contaminated.

All vessels in which milk has stood for any length of time should be thoroughly scalded before refilling.

Milk should not be kept warm in Thermos bottles or by any other method for any length of time. It favors the growth of bacteria. Even when pasteurized milk is so kept it permits the spores to develop. When milk is pasteurized all living bacteria are killed, but the spores ("the eggs" from which bacteria develop) are not destroyed.

The nurse's hands should be thoroughly scrubbed before preparing milk mixtures and before feeding the children.

\section{BOTTLES AND NIPPLES}

The best style of bottle is that which can be most easily cleaned. On no account should bottles with any complicated apparatus be allowed. The cylindric bottles with wide mouths are generally preferred. The best nipples are those of plain black rubber which slip over the 
neck of the bottle. Those with long rubber tubes going to the bottom of the bottle should not be used, as it is practically impossible to keep them clean. The hole in the nipple should be large enough to allow the milk to drop rapidly when the bottle is inverted, but not so large as to permit the milk to run through in a stream.

The bottles should first be rinsed with cold water, then washed with hot soapsuds and a bottle-brush. When not in use they should stand full of water. Before the milk is put into them they should lie for twenty minutes in boiling water. After the bottles have been sterilized they should not remain uncovered, but should be stoppered immediately with sterile cotton.

Nipples should be boiled for five minutes daily, and when not in use they should be kept in a receptacle containing a saturated solution of boric acid.

To prevent nipples from collapsing, the nursing bottle should be held at such an angle so that the nipple is constantly filled with milk. If for any reason a nipple is removed while feeding, do not put it down carelessly; it is better to drop it into the receptacle containing boric acid.

\section{RULES FOR FEEDING}

A child should not be more than twenty minutes taking its food, and should not be allowed to sleep with the nipple in its mouth. The bottle should be placed so that the child sucks milk, and not air.

The bottle of milk should always be warmed to a temperature of $100^{\circ} \mathrm{F}$. before feeding. This is done by placing the bottle in water, which is heated until the desired temperature is obtained. One of the handiest and 
quickest methods of heating milk is by using a "Bubble quick." 'This is a patented apparatus which can be obtained in most of the large cities.

If there is regurgitation immediately after feeding, sit the infant upright. Often there will be an eructation of gas which will eliminate this tendency.

A child should never be jumped up and down or rocked while it is being fed or immediately afterward.

Children should be kept quiet after their evening meals to avoid the occurrence of night terrors.

Schedule for Feeding Healthy Infants During the First Year.

$\begin{array}{llll}\text { Number of } & \text { Interval be- Night feed- } & \text { Ounces } & \text { Ounces } \\ \text { Age. } & \text { feedings in tween meals ings: ro P.M. for one } & \text { for twenty- }\end{array}$
$\begin{array}{llll}\text { twenty-four by day. } & \text { to } 7 \text { A.M. } & \text { feeding. four hours. }\end{array}$

$3^{\text {d to }} 7$ th day....... Io Hours.

2d to 3 d week...... Io

$4^{\text {th }}$ to $5^{\text {th }}$ week.... 9

6 th week to $3 \mathrm{~d}$ month. 8

$3^{\text {d to }} 5^{\text {th month..... } 7}$

$5^{\text {th }}$ to $9^{\text {th }}$ month. . . 6

$9^{\text {th }}$ to 12 th month.... 5

\begin{tabular}{|c|c|c|c|}
\hline 2 & 2 & I to $I \frac{1}{2}$ & Io to 15 \\
\hline 2 & 2 & I $\frac{1}{2}$ to 3 & I 5 to 30 \\
\hline 2 & I & $2 \frac{1}{2}$ to $3 \frac{1}{2}$ & 22 to $3^{2}$ \\
\hline $2 \frac{1}{2}$ & I & 3 to $4 \frac{1}{2}$ & 24 to 36 \\
\hline 3 & I & 4 to $5 \frac{1}{2}$ & 28 to 38 \\
\hline 3 & o & $5 \frac{1}{2}$ to 7 & 33 to 42 \\
\hline $3 \frac{1}{2}$ & ○ & $7 \frac{1}{2}$ to 9 & $\begin{array}{l}37 \text { to } \\
\text { lt.) }\end{array}$ \\
\hline
\end{tabular}

Usually the child's food in health should be increased in strength just as fast as the child's digestion will permit. An infant much above the average in weight must have its food graded accordingly. With this knowledge artificial feeding in health resolves itself into an easy problem.

Indications for Varying Mixture.-In regard to the exact indications when the fats, sugar, and proteids of milk are to be varied in infant feeding, much is yet to be learned; however, the following are the chief points:

Sugar.-If the sugar is too low, the gain in weight is slower than when it is furnished in proper amounts.

Excess of sugar is shown by colic, or thin, green, and 
very acid stools, which cause irritation of the buttocks. Sometimes eructations and regurgitations of small quantities of food take place.

Fat.-Excess of fat is shown by vomiting or regurgitation of food in small quantities, usually one or two hours after feeding; sometimes by frequent stools which are almost normal. There may be fat lumps in the stools.

Too little fat causes constipation, and dry and hard stools.

Proteids.-Excess of proteids is shown by the presence of curds in the stools, by colic, constipation, and vomiting.

Excess in quantity of milk given at a feeding causes immediate regurgitation.

It is not practicable to modify the milk so as to meet every temporary symptom of discomfort an infant may have. The general rules are:

If they are not gaining in weight without special signs of indigestion, increase the proportions of all the ingredients.

If there is habitual colic, reduce the proteids.

For frequent vomiting, soon after eating, reduce the quantity.

For the regurgitation of sour masses of food reduce the fat and also sometimes the proteids.

For obstinate constipation increase both fat and proteid.

\section{THE USE OF FOODS OTHER THAN MILK DURING THE FIRST YEAR}

The addition of other foods should be deferred until after the eighth or ninth month. Starch can then be added, usually in the form of barley. As starch is added, 
sugar should be gradually withdrawn. The only other thing to be advised during the first year is beef juice; this may be given after the tenth or eleventh month to weak infants; at first only $\frac{1}{2} \mathrm{oz}$. daily; later $3 \mathrm{oz}$.

In difficult cases of feeding the problem is essentially the same: that the food must be adapted to an infant whose powers of digestion are very feeble and easily disturbed.

The general principles to be followed in these cases are to give larger quantities of diluted food after three months of age, to feed at more frequent intervals, and since the proteids give the most trouble, the fats coming next, to reduce the strength of these elements. Sugars rarely cause trouble; therefore, it is seldom necessary to reduce them. Hence such formulæ as 2, 6, .75 are often seen.

Another plan that may be followed when the infant has great trouble in digesting the proteids of cows' milk is to peptonize it, or to add some such ingredients as oatmeal, arrowroot, or barley. These may take the place of some of the plain boiled water of the formulæ previously given. The number of cases that cannot be managed by simply varying the elements of cows' milk is small.

The scales are the best means of deciding whether a child is progressing favorably. At first the gain in weight will be slow-2 or 3 oz. a week; later, however, they should gain about $8 \mathrm{oz}$. a week. For those children who do not thrive on intelligent modification of cows' milk sometimes the substitution, for a short period, of condensed milk succeeds; sometimes they thrive upon sterilized milk, malted soups, or broths and bean flour, withholding the milk for a time. In every instance the 
general principle must be to begin with something which the child can digest and assimilate, and return to the usual proportions of the milk ingredients gradually, but just as soon as possible.

Most proprietary foods are composed almost entirely of carbohydrates and are insufficient in fats.

Throughout childhood, in all acute febrile diseases, the rule should be less food and more water.

When a child for any reason refuses to take its food, and there is danger of death from inanition, gavage should be practised.

In acute diarrhea in infancy stop the mixture and give barley water for twenty-four hours; purge and return slowly to normal mixture.

\section{FEEDING DURING AND AFTER THE SECOND YEAR OF LIFE}

The average child, when it reaches the age of twelve months, can take plain milk without any addition of water, or milk with the addition of small quantities of water. The child should weigh about twenty pounds, be about twenty-nine inches in length, have six teeth, and during the second year begin to walk around a chair.

The child should be vaccinated during the second year, if this has not been done earlier. It should be taught to make known when it desires to urinate or to have a bowel movement, and to have them at convenient and regular periods five or six times a day. The foreskin of a boy should be retracted daily until there is no trouble in pulling it back. This prevents trouble later.

A child should have a healthy complexion, a clean tongue, and well-digested bowel movements. 
To feed children too often, with too many kinds of food and with too little milk, during the second year, is a mistake.

A child of twelve months should be fed five times a day, say at 6 o'clock in the morning, Io, I, 5 and 9 P. M. Often a simple milk diet will be sufficient, the milk being given plain or with small quantities of water added. Eight ounces at a feeding is enough. In addition to this $4 \mathrm{oz}$. (eight tablespoonfuls) of gruel and two teaspoonfuls of orange juice can be added to the I o'clock meal. Orange juice is always a good thing to give children, as it prevents scurvy. It can be given after the age of six months. The best gruel is oatmeal. Do not give this instead of milk, but in addition to the milk.

At twelve months a child's diet should be:

6 A. M., 8 oz. of milk.

Io A. M., $8 \mathrm{oz}$. of milk.

I P. M., $8 \mathrm{oz}$. of milk, $4 \mathrm{oz}$. of gruel, $\frac{1}{2} \mathrm{oz}$. of orange juice.

5 P. M., $8 \mathrm{oz}$. of milk.

9 P. M., 8 oz. of milk.

Other food than milk should be taken from a spoon.

The teeth that a child has at twelve months are not chewing teeth; therefore, no solid food should be given. Children do not have chewing tecth until they are twenty months old.

At fifteen months of age a soft boiled egg can be added at the I o'clock meal, and the gruel increased to $6 \mathrm{oz}$., given twice a day, say at the 8 A. M. and the 5 P. M. meals.

At fifteen months a child's diet should be as follows: 
8 A. M., 8 oz. of milk, 6 oz. of gruel.

Io A. M., $8 \mathrm{oz}$. of milk.

I P. M., 8 oz. of milk, a soft boiled egg, $\frac{1}{2} \mathrm{oz}$. orange juice.

5 P. M., 8.oz. of milk, 6 oz. of gruel.

9 P. M., 8 oz. of milk.

A child should not have potatoes until it is eighteen months old. Rice in small quantities may be given at intervals after fifteen months.

At eighteen months clear chicken or mutton soups may be added to the mid-day meal; also dried bread and a little butter. The other meals should be the same as at fifteen months. Do not give beef juice except when the child is in poor health, at which time it acts as a tonic. It will do no harm, but, on the other hand, it will do no good in health.

At twenty-one months a child can digest meat in small amounts. Scraped beef from the inside of rare steak is the best, a tablespoonful of this may be added to the mid-day meal.

The diet at twenty-one months will be as follows: butter.

8 A. M., 8 oz. of milk, 6 oz. of gruel, soft boiled egg, bread and ro A. M., 8 oz. of milk.

I P. M., $6 \mathrm{oz}$. of clear soup, $\frac{1}{2} \mathrm{oz}$. of scraped beef, orange juice, bread and butter.

5 P. M., 8 oz. of milk, 6 oz. of gruel, bread and butter.

9 P. M., 8 oz. of milk.

The milk is discontinued when the meat is given.

Always give a child water between each meal; the best time is one hour before feeding. Let it have all it wishes.

Milk should be pasteurized if scarlet fever is epidemic.

At the beginning of the third year the 9 P. $\mathbf{M}$. bottle of milk can be discontinued. At this age a child can go without food for twelve hours and it is a better plan to have it retire with an empty stomach. 
The orange juice should be continued; vegetables may be added, but should always be put through a colander and served as a pulp. Potatoes, peas, squash, and spinach may be used. A dessert, such as junket, may be added.

The diet during the third year will be as follows:

8 A. M., orange juice, ro oz. of milk, $6 \mathrm{oz}$. of gruel, soft boiled egg, bread and butter.

Io A. M., $8 \mathrm{oz}$. of milk.

I P. M., 6 oz. of soup, meat, vegetables, bread and butter, dessert. 5.30 P. M., 10 oz. of milk, $6 \mathrm{oz}$. of gruel, bread and butter.

After three years of age three meals are sufficient. The food may be slowly increased in amount with a few additions until ten years of age. The diet will be as follows:

Breakfast: Fruit, cereals, milk, bread and butter, one or two eggs.

Dinner: Soup, meat, bread and butter, vegetables, and dessert.

Supper: Cereals, milk, bread and butter.

The foods which may be given during this period are milk, cream, eggs, rare beef, mutton, lamb, white meat of chicken, and well-cooked fish.

Vegetables: Potatoes, asparagus tips, spinach, stewed celery, string beans, and fresh peas.

Cereals: The best are the hominy grits, split wheat, and oatmeal. They should be cooked at least six hours. The prepared cereals should be cooked about four times as long as the directions say.

Broths and soups.

Bread and biscuits.

Desserts: Junket, plain custards, rice pudding without 
raisins, and, not oftener than once a week, good ice cream.

Fruits: Oranges, baked apples-never raw apples until ten years of age, and then with caution. Jams and preserved fruits cause trouble.

Do not give fat or greasy food to children. Only the meats and vegetables mentioned are feasible. Also hot bread, griddle cakes, all nuts, candies, pies, tarts, salads, jellies, pastry of every description, tea, coffee, cocoa, beer, cider, bananas, and dried fruits should never be given to children.

A light lunch at I0.30 or I I o'clock spoils the appetite for dinner. It is better not to change the child's food during the hot weather.

\section{ADJUNCTS TO FEEDING}

The principal adjuncts to feeding during childhood are rest, exercise, and ventilation.

Rest.-Young children about two years of age require a great deal of rest. They should sleep twelve hours at night, with a nap in the morning and one in the afternoon; the afternoon nap should be at least two hours in length. As the child becomes older the morning nap may be dropped. At ten years of age they should have at least from ten to eleven hours rest a day.

Children should never be too active in the afternoon, as this is frequently the cause of bed-wetting.

Exercise.-During the first year the baby gets enough exercise from waving its legs and arms about. During the second year a "baby jumper" is helpful. From it the child obtains sufficient exercise without hurting and unduly tiring itself. If the child walks from room to 
room or about the chairs it should rest often to preserve the arch of the foot. Boys usually obtain sufficient exercise from their play. Exercise is the best cure for constipation.

Ventilation.-Give children all the fresh air they can get, do not have their clothing too tight, and have a window open in the room at night.

An infant requires rooo cubic feet of fresh air. Older children should have between 700 and 800 cubic feet. 


\section{CHAPTER XIX}

\section{THERAPEUTICS}

IN the treatment of children more can be accomplished by good hygienic surroundings, careful feeding, and proper nursing than by the administration of drugs. Drugs are necessary under certain conditions, when it is better to give divided doses frequently than a large amount at one time.

Drugs well borne by children include alcoholic stimulants, which should be diluted eight times before administration, quinin, calomel, iodids, cod-liver oil, bromids, chloral, and belladonna.

Belladonna often causes an erythema or redness of the skin even when given in small quantities, but this does not necessarily mean that the drug is producing deleterious effects.

Chloral should be given by the rectum. When given by the mouth it causes irritation of the mucous membranes.

Drugs poorly borne by children include opium, usually given in the form of Dover's powder, salicylates, iron, and acids. Children are more susceptible to opium than adults. The other drugs mentioned have a tendency to derange the digestion. Mixtures containing arsenic should be diluted with at least eight parts of water when administered. 


\section{RULES FOR DOSAGE IN CHILDHOOD}

Several rules for dosage in childhood have been devised, founded on the fact that drugs influence the human organism somewhat in proportion to the body weight.

Young's Rule.-Add twelve to the age of the child and divide the sum into the age. This gives the proportionate quantity of an adult dose. Thus, the age of a child being two years, two plus twelve would be fourteen, and fourteen divided into two would be $\frac{2}{14}$ or $\frac{1}{7}$ of the adult dose, being the proper dose for a child of two years.

Crowling's Rule.-Divide the age of the child at the following birthday by twenty-four, and the result is the proportionate dose for that child. Thus, the following birthday of a child being four years, $\frac{4}{24}$ or $\frac{1}{6}$ of the adult dose would be the proper quantity for a child at three years.

\section{THE THERAPEUTIC LIMIT}

The therapeutic limit of a drug is the furthest point to which a drug can be pushed, with safety, in the treatment of a disease.

The therapeutic limit of the following drugs is:

Aconite. Tingling of the mucous membrane of the mouth and lips and a weak, compressible pulse.

Antipyrin. Cyanosis, languor, and a weak pulse.

Arsenic. Nausea and diarrhea. Puffiness under the eyes.

Aspirin. Ringing in the ears.

Belladonna. Dilation of the pupil, dryness of the mouth, and a rapid, corded pulse.

Bromids. Mental torpor and an acne rash (bromism). 
Carbolic acid. Smoky urine.

Cimicifuga. Frontal headache.

Colchicum. Serous diarrhea.

Digitalis. A slow, full pulse.

Iodids. Headache, coryza, and sore throat.

Mercury. Salivation, sore gums, and fetid breath (ptyalism).

Oil of Wintergreen. Ringing in the ears.

Opium. Contraction of the pupils and sleep.

Phosphorus. Matchy taste.

Quinin. Ringing in the ears.

Salicylates. Ringing in the ears.

Salol. Ringing in the ears.

Strychnin. Stiffness of the muscles of the neck, twitching of the muscles, and nervousness.

Sulphonal. Pinkish urine.

Tartar Emetic. Nausea and a slow pulse.

Thyroid Extract. Loss of weight and strength, fever, and a rapid pulse.

\section{CONTRA-INDICATIONS}

Aconite. Contra-indicated in weak heart.

Alcohol. Contra-indicated in typhoid if odor is present on breath.

Chloral. Contra-indicated in hypertrophied heart and disease of heart muscle.

Chloroform. Contra-indicated in heart disease.

Digitalis. Contra-indicated in hypertrophied heart and disease of heart muscle.

Ether. Contra-indicated in disease of the bronchi and lungs.

Hyoscin. Contra-indicated in sore throat. 
Iodid. Contra-indicated in cavity formation in phthisis.

Mercury. Contra-indicated in inflammations of a serous membrane with serous exudate.

Nitrous Oxid Gas. Contra-indicated in aneurysm and arteriosclerosis.

Opium. Contra-indicated in Bright's disease.

Quinin. Contra-indicated in middle-ear disease.

Strychnin. Contra-indicated in inflammations of the spinal cord.

Tartar Emetic. Contra-indicated in infancy.

Thyroid Extract. Contra-indicated in exophthalmic goiter.

Tonics (Bitter). Contra-indicated in inflammations of gastro-intestinal tract.

Veratrum Viride. Contra-indicated in gastric inflammations and weak heart.

\section{DOMINANT ACTION OF DRUGS}

Aconite depresses the heart directly.

Amyl nitrite depresses the motor portion of the spinal cord.

Bromids depress the motor portion of the spinal cord.

Chloral depresses the motor portion of the spinal cord.

Digitalis stimulates every portion of the circulation.

Strychnin stimulates the motor portion of the spinal cord.

\section{DRUGS WHICH QUICKEN THE PULSE}

Alcohol.

Atropin.
Ammonia.

Ether.

Nitroglycerin. 


\section{DRUGS WHICH SLOW THE PULSE}

Aconite.

Digitalis.

Chloroform.

Opium.

Veratrum Viride.

DRUGS WHICH RAISE BLOOD PRESSURE

Alcohol.

Ammonia.

Atropin.
Cocain.

Digitalis.

Ergot.

Strychnin.

CHARACTERISTIC PULSES

Aconite. Slow, weak pulse.

Amyl Nitrite. Rapid, soft pulse.

Digitalis. Slow, full pulse.

Opium. Slow, full pulse.

Veratrum Viride. Slow, weak pulse.

DRUGS WHICH DILATE THE PUPIL

Belladonna.

Cocain.

DRUGS WHICH CONTRACT THE PUPIL

Eserin.

Opium.

\section{DRUGS WHICH CAUSE SKIN REACTIONS}

Erythematous Eruptions :

Antipyrin.

Belladonna (resembles scarlet fever).

Chloral.

Quinin. 
Acneiform Eruption:

Arsenic. Bromids. Iodids.

Drugs causing Cyanosis:

Antipyrin.

Potassium chlorate.

Hydrocyanic acid.

Nitrites.

\section{DRUGS WHICH COLOR THE URINE}

Carbolic acid. Smoky urine.

Creosote. Olive-green urine.

Methylene-blue. Blue-green urine.

Resorcin. Olive-green urine.

Salicylates. Olive-green urine.

Santonin. Yellow urine.

Sulphonal. Pinkish urine.

Thymol. Olive-green urine.

Turpentine and eucalyptus give an odor of violets to urine.

\section{DRUGS WHICH COLOR THE STOOLS}

Bismuth. Black stool.

Hematoxylin. Red stool.

Iron. Black stool.

Silver. Black stool. 


\section{DRUGS OF THE U. S. PHARMACOPEIA MOST COMMONLY EMPLOYED IN CHILDREN'S DISEASES, TOGETHER WITH THEIR DOSES FOR CHILDREN TWO YEARS OLD}

Acetanilid, gr. ss-j.

Aceta.

Acetum opii, $\mathbf{M}_{\text {ss-j. }}$. scillæ, $m_{i-v}$.

\section{Acida.}

Acidum aceticum dil., $m_{v-x v}$. carbolicum, gr. $\frac{1}{8}$. gallicum, gr. ss-ij. hydrocyanicum dil., $m_{\frac{1}{8}-\frac{1}{4}}$. hydrochloricum dil., $\mathrm{m}$ i-v. nitricum dil., $m i$ iij . nitrohydrochloricum dil., $\mathrm{m}$ i-v. phosphoricum dil., $\mathrm{mi}$-v. salicylicum, gr. j. sulphuricum dil., $m i-v$. aromaticum, $\mathrm{mi}_{\mathrm{i}-\mathrm{v} \text {. }}$ tannicum, gr. ss-ij.

\section{Etherea.}

Æther, $m_{\text {ii }}-\mathrm{x}$.

Chloroformum, $\mathrm{mi}_{\mathrm{i}-\mathrm{v} \text {. }}$

\section{Ammonia.}

Ammonii bromidum, gr. i-v. carbonas, gr. ss-j. chloridum, gr. i-v.

\section{Antimonium.}

Antimonii et potassii tartras, gr. $\frac{1}{48}-\frac{1}{4}$.

Antipyrin, gr. ss-ij.

Aspirin, gr. ss-iss.

\section{Aquæ.}

Aqua ammoniæ. (External.) camphoræ, $\mathrm{f} z \mathrm{j}$. cinnamomi, $\mathrm{f} / \mathrm{i}-\mathrm{ij}$. menthæ piperitæ, $f Z^{i-i j}$. rosæ. (External.)

\section{Argentum.}

Argenti nitras, gr. $\frac{1}{64}-\frac{1}{32}$. nitras fusa. (External). Atropin Sulph., gr. $\frac{1}{8} \frac{1}{\delta} \sigma-\frac{1}{4} \frac{1}{0} \sigma$.
Bismuthum.

Bismuthi subcarbonas, gr. i-v. subnitras, gr. i-v.

Calcium.

Creta præparata, gr. ii-x.

Testa præparata, gr. ii-x.

Carbo Ligni, gr. i-v.

Cerata. (External.)

Ceratum canthar. cetacei. ext. canth. plumbi sub. resinæ. resinæ com. sabinæ. saponis. zinci carbon.

Chartæ. (External.) Charta sinapis.

Chloral, gr. i-v.

Codein, gr. $\frac{1}{48}-\frac{1}{24}$.

Collodium. (External.)

Collodium cum cantharidê. flexile.

Confectiones.

Confectio sennæ, gr. $\mathrm{x}$-xx.

Decocta.

Decoctum hæmatoxyli, f $z^{i-i j}$. hordei, $\mathrm{f} \tilde{\mathbf{z}} \mathrm{i}-\mathrm{iv}$. quercus. (External.)

Digitalin, mess-v.

Emplastra. (External.)

Emploastrum assafotidæ. belladonnæ. hydrargyri. opii. 
Emplastra. (External.)

Emploastrum picis burgundicæ. cum cantharidê. resinæ. saponis.

\section{Extracta.}

Extractum belladonnæ, gr. $\frac{1}{32}-\frac{1}{16}$. cinchonæ, gr. i-iv. colocynthidis C., gr. -j. gentianæ, gr. $\frac{1}{2}-\mathrm{j}$. glycyrrhizæ, gr. i-v. hæmatoxyli, gr. i-iv. hyoscyami, gr. $\frac{T}{16}-\frac{1}{4}$. krameriæ, gr. $\frac{1}{2}$-ij. malti, $m_{x v-f} z^{\text {ss. }}$. nucis vomica, gr. $\frac{1}{18} \frac{1}{24}$. taraxaci, gr. ii-x.

\section{Extracta Fluida.}

Extractum buchu fluid., $\mathrm{m}$ ii-v. cascara sagrada fld., $m_{\text {ii }}-\mathrm{x}$. cimicifugæ fluid., $\mathbf{M}_{\text {iv-viij. }}$ ergotæ fluid., $m_{i-i j}$. gelsemii fluid., $m \frac{1}{2}$. grindeliæ fluid., (External.) pilocarpi fluid., $m x$. pruni virg. fluid., $m x$. rhei fluid., $m_{i-v}$. sennæ fluid., $m x$-xxx. spigeliæ et sen. fluid., $\mathrm{f} 3 \mathrm{j}$. uvæ ursi fluid., $m_{\text {ii-v. }}$ valerianæ fluid., $m$ ii-x.

\section{Ferrum.}

Ferri citras, gr. ss-ij. et ammonii citras, gr. ss-ij. et potassii tartras, gr. ss-ij. et quinine citras, gr. ss-ij. lactas, gr. ss-ij. pyrophosphas, gr. $\frac{1}{4-j}$. subcarbonas, gr. i-ij. sulphas exsiccata, gr. $\frac{1}{-j}$. Ferrum reductum, gr. ss-ij.

\section{Glycerita, (External.)}

Glycerinum acidi carbolici. gallici. tannici.

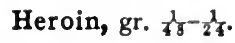

Hydrargyrum.

Hydrargyri chloridum corros., gr. $\frac{1}{10} \sigma^{-}$ mite, gr. $\frac{1}{24}$-iss.

Hydrargyrum cum creta, gr. ss-iss.

\section{Infusa.}

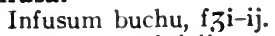
calumbæ, $f z^{i-i j}$. digitalis, $m_{x-f} Z^{\text {ss. }}$. lini, f $\tilde{z}_{s s-i j}$.

Kamala, gr. v-xv.

\section{Linimenta. (External.)}

Linimentum ammoniæ. calcis. camphoræ. cantharidis. chlor of ormi. plumbi subacetatis. saponis. terebinthinæ.

\section{Liquores.}

Liquor acidi arseniosi, $m i-i i j$. ammonii acetatis, $\mathrm{f} \mathbf{Z}^{\mathrm{ss}-\mathrm{j}}$. arsenici et hydrarg. iodidi, Mss-j. calcis, $\mathrm{f}^{\mathrm{i}-\mathrm{f}} \mathrm{f}^{\mathrm{iij}}$. ferri nitratis, $M i$-iij. subsulphatis, $\mathrm{M}_{\mathrm{ss}-\mathrm{j} \text {. }}$ magnesii citratis, $\mathbf{f}^{\text {ii }}$ - $\bar{z}^{\text {sss. }}$. pepsini, $f \tilde{z} s s-f ; j$. plumbi subacetat. dil. (External.)

potassii arsenitis, $\mathrm{m}_{\mathrm{i}-\mathrm{iij} \text {. }}$ citratis, $\mathrm{f} z \mathrm{ss}-\mathrm{j}$. sodæ chloratæ. (External.)

\section{Magnesium.}

Magnesia, gr. v-gr. xl.

Magnesii carbonas, gr. v-gr. $x l$. sulphas, gr. $\mathbf{v}-\mathbf{x v}$.

Manna, gr. $\mathrm{xx}-3 \mathrm{j}$.

Mellita. (External.)

Mel despumatum. rosæ. boracis. 
Misturæ.

Mistura ammoniaci, $\mathbf{f}^{\mathrm{ss}}-\mathrm{ij}$.

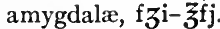

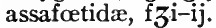

chloroformi, $\mathbf{f} \mathbf{z}$-ij.

cretæ, f $\mathbf{z}_{\mathrm{ss}} \mathrm{j}$.

ferri comp., f $z^{i-i j}$.

ferri et ammonii acetatis, $f \mathbf{z}$ ss-j.

glycyrrhizæ comp., $m_{x v-f} z_{\text {ss. }}$

potassii citratis, ${ }^{\mathrm{ss}-\mathrm{j}}$.

rhei et sodæ, f $Z^{s s-j}$.

Morphina.

Morphinæ acetas, gr. $\frac{1}{48} \frac{1}{30}$. murias, gr. $\frac{1}{48}-\frac{1}{3}$.

sulphas, gr. $\frac{1}{48}-\frac{1}{30}$.

Moschus, gr. i-ij.

Mucilagines.

Mucilago acaciæ, ad lib. sassafras medullæ, ad lib. tragacanthæ, ad lib. ulmi, ad lib.

\section{Nitroglycerin, gr. $\frac{1}{500}$.}

Olea.

Oleum chenopodii, $M$ ii-iv. cinnamomi, $m_{\frac{1}{4}}$. gaultheriæ, $m_{\mathrm{i}-\mathrm{ij} \text {. }}$ menthæ piperitæ, $\mathrm{m}_{\mathrm{ss}-\mathrm{j}}$. morrhuæ, $m_{x v-f} \mathbf{j}$. olivæ, $f z^{i-i j}$. ricini, f $3 \mathbf{s s}-\mathrm{ij}$. succini. (External.) terebinthinæ, $m_{\text {ii-v. }}$.

Oleoresinæ.

Oleoresina aspidii, $m_{\mathbf{v}-\mathrm{xx}}$.

Opium, gr. $\frac{1}{10-\frac{1}{8}}$.

Pelletierine tannas, gr. i-v.

Pepo, $3^{\mathrm{i}-\mathrm{ij} \text {. }}$

Petrolatum (External).

Phenacetin, gr. ss-j.

Phosphorus, gr. $\frac{1}{60}-T^{\frac{1}{1}}$.

Plumbum.

Plumbi acetas, gr. $\frac{1}{8}-\frac{1}{6}$.
Potassium.

Potassii acetas, gr. ii-v. bicarbonas, gr. ii-v. bitartras, gr. $\mathrm{x}-\mathrm{xv}$. bromidum, gr. ii-v. chloras, gr. ii-v. citras, gr. ii-v. et sodii tartras, $g r . \mathbf{x v}-\mathbf{z j}$. iodidum, gr. ss-iij. nitras, gr. iss-viij. permanganas. (External.)

\section{Pulveres.}

Pulvis aromaticus, gr. i-ij. glycyrrhizæ comp., gr. iv-viij. ipecacuanhæ et opii, gr. $\frac{1}{4}$-iss. rhei comp., gr. $\mathbf{v}-\mathbf{x}$.

Quinina.

Quininæ bisulphas, gr. ss-iv. sulphas, gr. ss-iv. valerianas, gr. $\frac{1}{4}-$ ss.

Resinæ.

Resina jalapæ, gr. $\frac{1}{4}$-ss. podophylli, gr. $\frac{1}{64}-\frac{1}{48}$. scammonii, gr. $\frac{1}{4}$-iss.

Rhe um, gr. ss.-ij.

Salol, gr. ss-iss.

Santoninum, gr. $\frac{1}{4}$-ss.

Scammonium, gr. $\mathrm{i}-\mathrm{ij}$.

Senna, gr. iii-v.

Sinapis (Emetic), gr. viii-xx.

\section{Sodium.}

Sodii acetas, gr. ii-v. arsenias, gr. $\frac{1}{6} \sigma^{-\frac{1}{64}}$. bicarbonas, gr. ii-v. boras. (External.) bromidi, gr. i-v. salicylici, gr. ss-iss.

\section{Spiritus.}

Spiritus ætheris comp., $\mathrm{m}$ ii-x. nitrosi, $M_{v-x x}$ ammoniæ aromat., Mii-v. camphoræ, $M$ ss-iv. chloroformi, $\mathrm{mi}$-v. 


\section{Spiritus.}

cinnamomi, $m i-i j$.

frumenti, $m_{v-f . j j}$.

juniperi comp., $\mathfrak{m}_{\mathrm{v}-\mathrm{xv}}$.

menthæ piperita, $m_{s s-i j}$.

vini gallici, $m_{v-f} \mathbf{z}$.

\section{Strychnina.}

Strychnina sulphas, gr. $\frac{1}{15} \sigma^{\frac{1}{10}}$.

Sulfonal, gr. iss.

Sulphur.

Sulphur præcipitatum, gr. $\mathrm{x}-\mathrm{xv}$.

\section{Syrupi.}

Syrupus acacia, ad lib.

allii, $\mathbf{z}^{\text {ss }}-z^{\mathrm{ij}}$.

ferri iodidi, Mii-v.

ipecacuanha, $m_{\mathrm{ii}-\mathrm{f}} \boldsymbol{j} \mathrm{j}$.

krameria, $m_{x x-f} \mathbf{j}$.

lactucarii, $M_{x x}-\mathrm{f} \tilde{\jmath} \mathrm{j}$.

limonis, ad lib.

pruni virginianæ, $m_{x v-f} \tilde{z}$ ss.

rhei, f $\tilde{z}$ ss-j.

aromat., $\mathrm{f} \overline{\mathrm{ss}} \mathrm{j}$.

sarsaparillæ comp., $\mathfrak{m}_{\mathrm{xv}-\mathrm{f}} \mathfrak{Z}_{\mathrm{ss}}$. scillæ, $M_{\text {ii-vj. }}$.

comp., Mii-vj.

senegx, $m_{\text {ii }}-\mathrm{v}$.

tolutani, $m$ ii-vj.

zingiberis, $m_{v-x}$.

\section{Tincturæ.}

Tinctura aconiti, $m_{\frac{1}{4}}$-ss. belladonnæ, $m i-i j$. calumber, M iii-xv. cannabis indicæ, $\mathrm{mi}-\mathrm{ij}$. cardamomi comp., $m_{v-x v}$. catechu comp., $m_{v-x v}$. cinchonæ comp., $\mathfrak{m}_{\mathrm{x}-\mathrm{xv}}$. cinnamomi, $M$ ii-xv. colchici, $m i-i i j$. digitalis, $\mathfrak{m}_{\mathrm{ss}-\mathrm{iij}}$. ferri chloridi, Mi-v. gelsemii, $m i-i j$. gentianæ comp., $m_{v-x v}$ guaiaci ammon, $m_{v-x v}$. hyoscyami, $M i-i v$. iodi, Mi-iv.

ipecac. et opii, $m_{\frac{1}{4}}$-iss.

kino, $m$ ii-xx.

kramerix, Mii-xx.

lavendulæ comp., $m_{\mathbf{v}-\mathbf{x x} \text {. }}$

\section{Tincturæ.}

nucis vomicæ, $m_{\text {ss-ij. }}$.

opii, Mi-iij.

camphorata, $\mathbf{M}_{\mathrm{v}-\mathrm{xx}}$.

deodorata, $m i$-iij.

rhei dulcis, $m_{\mathbf{v}-\mathbf{x}}$.

saponis viridis. (External.)

scillæ, $m$ i-v.

strophanthus, $m_{\text {ss }-j .}$.

valerianæ ammoniata, $\boldsymbol{M}_{\mathbf{v}} \mathbf{x v}$. zingiberis, $M_{\text {ii-vij. }}$

Trional, gr. iss.

Unguenta. (External.)

Unguentum acidi carbolici. acidi tannici.

aqua rosx.

belladonnæ.

cantharidis.

creasoti.

diachylon.

gallæ.

hydrargyri.

ammoniati.

iodidi rubri.

nitratis.

oxidi flavi. rubri.

iodi ichthyol.

mezerei.

picis liquidæ.

plumbi carbonatis.

iodidi.

potassii iodidi.

stramonii.

sulphuris.

iodidi.

veratrinæ.

zinci oxidi.

Veronal, gr. iss.

Vina.

Vinum ergotæ, $m_{v-x}$.

ferri amarum, $\mathcal{M} x x-f=3 s s$.

ipecacuanhæ, $m_{\text {ii-viij. }}$

opii, $m_{\text {ss-j. }}$.

rhei, $m_{v-f} z^{\text {ss. }}$

\section{Zincum.}

Zinci oxidum, gr. $\frac{1}{8}-$ ss.

sulphas, gr. $\frac{1}{8}-\mathrm{v}$.

valerianas, gr. $\frac{1}{8}-$ ss.

Zingiber.

Pulv. zingiberis, gr. $t$-ij. 
Normal Salt Solution.-One and one-half drams of sodium chlorid (ordinary table salt) are added to two pints of sterile water, thoroughly mixed with a sterile glass rod, and filtered into a sterile bottle.

If normal salt solution is to be used for hypodermoclysis it should be sterilized for a half hour on three successive days. In emergencies it may be boiled steadily for one hour. A sterile thermometer should be used to take the temperature of the solution.

\section{POISONS AND THEIR ANTIDOTES}

Acids, Mineral.-Alkalies in dilute solution; lime; whitewash; magnesia; soap. Quickness of administration essential. Avoid emetics and stomach-pump.

Acids, Vegetable.--Soap; soda or potassa in dilute solution; except for oxalic acid, for which give lime, whitewash, chalk, or magnesia.

Aconite.-Emetics; stomachpump; tannic acid; digitalis; laudanum; warmth; stimulation; recumbent position.

Alkalies and Their Salts.-Vinegar; weak acids; oil freely; opium.

Alum.-Albumen.*

Antimony and Its Salts.-Tannic acid; opium; external warmth.

Arsenic and Its Salts.--Emetics; stomach-pump; recently made hydrated sesqui-oxid of iron; (made by adding water of ammonia to solution of trisulphate of iron); magnesia; dialyzed iron and salt; oil or fat.
Atropin.-See Belladonna.

Belladonna.-Emetics; stomachpump; tannic acid; morphin hypodermically.

Bismuth and Its Compounds.Albumen; milk.

Bromin.--Soap; oils.

Cannabis Indica.-Emetics; lemon juice; quiet; if exhaustion, stimulants.

Carbolic Acid.--Stomach-pump; solution of saccharate of lime; sulphate of sodium; hot and cold douche.

Chloral and Chloroform.-Alternate hot and cold douche; artificial respiration; cardiac stimulants.

Cocculus Indicus.-E met i c s ; stomach-pump; at first, give opium; chloral; chloroform; later, stimulants. No chemical antidote.

* Albumen is furnished by whites of eggs. 
Colchicum.-Emetics; stomachpump; tannic acid; opium; stimulants.

Conium.-Emetics; st o m a chpump; artificial respiration; tannic acid; opium.

Copper and Its Salts.-Albumen; milk; calcined magnesia; yellow prussiate of potash.

Croton Oil and Other Drastic Purgatives.-Emetics; albuminous drinks; bismuth; external heat.

Cyanid of Potassium.-See $\mathrm{Hy}$ drocyanic Acid.

Digitalis.-Emetics; stomachpump; tannic acid; stimulants.

Gelsemium.-Same as Aconite.

Hydrocyanic Acid.-Alternate hot and cold douche; intravenous injection of aqua ammonix; atropin hypodermically.

Hyoscyamus.-Same as Belladonna.

Iodin.-Starch, freely; if this cannot be obtained, then soap.

Iron, Salts of.-Soap; dilute alkalies; albumen.

Lead, Salts of.-Emetics; stomachpump; alkaline sulphates; soap; albumen; milk; purge.
Mercury, Salts of (Bichlorid of Mercury).--Emetics; albumen; milk; wash out stomach.

Opium.-Stomach-pump; atropin hypodermically; coffee; flagellation; artificial respiration; electricity.

Phosphorus.-Emetics; sulphate of copper in small doses; crude oil of turpentine; stomach-pump; avoid oils or fats.

Potash Salts.-No distinct antidote.

Santonin.-Emetics; purges; stimulants; artificial respiration.

Silver, Salts of.-Common salt, freely; albumen.

Soda Salts. - No distinct antidote.

Stramonium.-Same as Belladonna.

Strychnin.-Emetics; stomachpump (at first only) tannic acid; chloral; bromid of potash; ether; chloroform; rest.

Veratrum Viride.-Same as Aconite.

Zinc, Salts of.-Albumen in the form of white of egg; carbonate of soda; milk, freely. 


\section{TREATMENT OF EMERGENCIES}

The following treatments are given so that the nurse may know what to do in cases of extreme emergency, where it is impossible to obtain a physician immediately and where delay might result in the death of the child.

In every emergency case send for the physician at once, and while awaiting his arrival do as much for the patient as can be done without exercising his prerogatives, and at the same time have everything prepared so that his orders may be anticipated and the patient receive immediate medication. When the nurse knows what orders to expect, the detail can be worked out before the physician arrives.

Asphyxia.-Give artificial respiration (see page 42I). Oxygen, atropin, $\frac{1}{40} \overline{\mathrm{gr}}$., and strychnin, $\frac{1}{6} \overline{0}$ gr., hypodermically, to a child four years of age, will probably be ordered.

Asthma.- Prompt relief often follows the inhalation of a few ${ }^{\circ}$ drops of chloroform.

Bites should be treated as open wounds and not cauterized. They should be thoroughly washed with hydrogen peroxid and a wet bichlorid dressing applied.

Burns.-The burned area should be covered with lint saturated with normal salt solution or carron oil, which is composed of equal parts of linseed oil and lime-water.

Chills.-The child should be surrounded with hotwater bottles and covered with a blanket. Hot drinks may be administered. If this plan of treatment is followed by a sweat, the skin should be sponged with water containing a tablespoonful of alcohol to the basin. The temperature should be taken. 
Collapse.-Place the child in a mustard tub at a temperature of $110^{\circ} \mathrm{F}$. for five minutes, vigorously rubbing the extremities and the skin surfaces during this time. After removal from the tub place it in a horizontal position, cover with warm blankets; heart and respiratory stimulants will probably be ordered.

Croup.-A sponge moistened with hot water may be applied to the throat, or the child may be placed in a hot bath or mustard tub at a temperature of $110^{\circ} \mathrm{F}$. If these simple remedies fail, an emetic will often bring relief, the best being the wine of ipecac administered in dram doses until effective; or a little powdered alum mixed with honey or molasses given in teaspoonful doses. In severe cases it may be necessary to resort to the inhalation of a few drops of chloroform. A croup tent with moist atmosphere is advantageous. An umbrella covered with a blanket may be substituted for the regulation croup tent.

Convulsions. - Epileptiform. - Measures should be taken to prevent the child from injuring itself. Something should be placed between the teeth to prevent biting of the tongue. An enema should be given.

Uremic.-The same precautions must be taken as in epileptiform convulsions. The inhalation of a few drops of chloroform may control the seizures until the physician arrives.

Reflex Convulsions.-The child should be placed in a mustard tub, at $110^{\circ} \mathrm{F}$., for five minutes.

Dislocations.-Apply cold to the joint.

Drowning.-The child should be forced to vomit to relieve the stomach of the swallowed water; this can be accomplished by pressure over the stomach. Artificial respiration should then be practised (see page 42I). 
Dyspnea.-Dyspnea may be due to a number of causes. If the child is suffering from heart disease, it should be propped up in bed. If due to some disease of the respiratory tract, a croup tent with a moist atmosphere may help; oxygen should be administered if at hand, or the child should be kept in a room filled with fresh air or even carried out of doors. If due to diphtheria, intubation will probably be necessary. Heart or respiratory stimulants will probably be ordered.

Epistaxis or Nosebleed.-The child should sit upright in the chair, the clothing should be loosened about the neck, firm pressure made over the bridge of the nose by holding it between the fingers, and ice should be applied to the bridge of the nose and back of the neck. Small pellets of ice may be introduced into the nostrils or held in the mouth. If this does not answer, plugging the nostrils with absorbent cotton may be resorted to. Compound tincture of benzoin, diluted lemon-juice, and adrenalin chlorid, I: 2000 , may be introduced into the nose. No astringent powders should be used locally on account of their tendency to produce sneezing, thus starting the nosebleed afresh.

Earache.-Douche the ear with warm water at a temperature of $110^{\circ} \mathrm{F}$. for about five minutes (see page 410). Then introduce a pledget of cotton saturated with sweet oil and a drop of laudanum.

Fainting.-Loosen the clothing; place the child on its back with the head on a lower level than the feet; use smelling salts and aromatic spirits of ammonia, ten drops in a tablespoonful of water for a child four years of age.

Foreign Bodies. - Eye.-If possible remove the body with a wisp of cotton; if it cannot be discovered turn the 
lid as described on page 230 , when, in most cases, it can readily be found.

Ear.-Nothing should be used except hot-water douchings; on no account attempt to probe with any instrument; the douching will be sufficient for all cases.

Nose.-Dounching the nose with warm water is usually sufficient, the nozzle of the syringe being placed in the nostril which is free.

Throat.-Often a sharp slap on the back will cause the expulsion of the foreign body. If it is impossible to recover the object and it seems to be lodged in the throat, send immediately for the physician. If it has been swallowed give the child bread and potatoes to eat, and a dose of oil.

Fractures.-If simple, apply a temporary dressing and splint to avoid unnecessary laceration of the tissues.

If compound (communicating with the air) wash thoroughly with a solution of bichlorid of mercury, I to 4000 , and apply a wet bichlorid or normal salt solution dressing. In fractures of skull, apply an ice-cap.

Heart Failure.-Symptoms of heart failure demand instant attention. Whenever the pulse becomes rapid, intermittent, and weak, or the child has sudden attacks of dyspnea, coldness of the extremities, or attacks of syncope, the physician should be immediately informed. While awaiting his arrival the child should be placed flat upon its back and not moved for anything. Mustard paste, made of equal parts of mustard and flour, may be applied to front of chest until there is a distinct redness, 15 minims of aromatic spirits of ammonia may be given in water by the mouth, if the child can swailow; hot-water bags may be placed about the extremities, and inhala- 
tions of ammonia given. (Be careful not to have concentrated ammonia nor to hold it continuously under the nose; pass it slowly backward and forward.) The physician will probably order a hypodermic injection of one of the following drugs: nitroglycerin, strychnin, digitalis, or whisky, all of which should be in readiness. Hypodermoclysis may be ordered and at times he may bleed the child.

Hemorrhage.-Hemoptysis (Spitting of Blood).-Morphin should be administered in profuse hemorrhages, the dose being $\frac{1}{32}$ gr. to a child four years of age. Icebag to chest.

Hematemesis (Vomiting of Blood).-An ice-bag should be placed over the stomach and all food by the mouth prohibited. If profuse, morphin, $\frac{1}{32}$ gr., to a child of four years can be administered hypodermically.

Typhoid.-The hemorrhage from the bowel should be treated by absolute rest, ice-bag to the abdomen, the food should be reduced to the minimum and of the mildest character. In severe cases the foot of the bed can be raised and morphin, dose $\frac{1}{32}$ gr. to $\frac{1}{16}$ gr. to a child of four years, should be administered hypodermically.

Laryngismus Stridulus.-Dash cold water on the face and neck in an attempt to break the spasm. Mustard tubs at a temperature of $1 \mathrm{I}^{\circ} \mathrm{F}$. may be resorted to, and in severe cases inhalations of chloroform.

Nervousness.-Warm baths at a temperature of $\mathrm{I} I 0^{\circ} \mathrm{F}$. will usually allay nervousness.

Pain can usually be relieved by the application of heat; that is, hot-water bag, turpentine stupe, etc.

Perforation.-Typhoid.-Absolute quiet; no food or liquids by mouth or rectum. The physician should be immediately notified. 
Appendicitis. - The same plan of treatment should be followed.

Poisons.-If possible wash out the stomach, give a large dose of oil, and administer the proper antidote (see page 387 ).

Prolapse of the Rectum.- The prolapsed portion of the bowel should be greased with vaselin, cold cloths applied, and gently pushed within the sphincter.

Respiratory Failure.-In respiratory failure there is great dyspnea, cyanosis, and signs of collapse. The physician should be immediately summoned; in the meantime, if the child shows great distress in breathing while in the recumbent posture, it should be propped up in bed, oxygen should be administered; gentle friction of the sides of the chest at times stimulates the respiratory muscles. He may order a mustard tub and hypodermic injections of one or more of the following drugs: atropin, caffein, strychnin, and nitroglycerin. In sudden attacks of great cyanosis a mustard tub is advantageous.

Shock.-Hot $\left(\mathrm{II}^{\circ} \mathrm{F}\right.$.) normal salt solution or hot coffee may be injected into the rectum; about a pint should be used. Heart and vasomotor stimulants will be ordered, like strychnin, ammonia, and digitalis.

Sunstroke.-The child's clothing should be removed and cool drinks administered. The child should be placed in the coolest part of the room, ice-bags applied to the head, and cool sponging given. At times it is necessary to give cold baths and cold irrigations of colon (see page 396). Stimulation should be resorted to if necessary.

Urine.-Suppression of the urine may be overcome by giving the child plenty of water to drink and administering sweet spirits of niter, dose ten drops in water every three 
hours to a child of two years, until the kidneys become active.

Retention of the urine at times may be overcome by a hot sitz bath or a warm tub. If this fails and the child is suffering, catheterization is necessary (see page 437).

Persistent Vomiting.-Usually a mustard plaster applied for ten minutes over the stomach will relieve the vomiting. No food should be given by the mouth. Cracked ice will relieve the thirst.

Wounds.-All wounds should be thoroughly cleansed (all the dirt removed, washed with peroxid of hydrogen or a solution of bichlorid of mercury, I : 4000) and covered with a wet bichlorid or sterile normal salt solution dressing.

\section{Therapeutic Measures Employed in Childhood}

\section{TO REDUCE TEMPERATURE}

Ice-cap.-Ice is placed in a canvas bag and beaten with a mallet until broken in small pieces. It is then transferred to a rubber ice-bag. Express all air from the bag by twisting the unfilled portion. Carefully apply metal cap; then cover with towel or gauze.

Sponge Bath.-The temperature of the water should be from $85^{\circ} \mathrm{F}$. to $90^{\circ} \mathrm{F}$. Equal parts of water an alcohol or water and vinegar can be used. The clothes should be removed from an infant with the exception of the diaper. In older children the portions of the body not being sponged may be kept covered. The sponging should be continued for five to fifteen minutes and the child then wrapped in a blanket without further dressing. The temperature of a child should be taken half an hour after sponging. 
Cold Pack.-The child is stripped of all its clothing, laid upon a blanket, and the entire trunk wrapped in a sheet which has been wrung out of water at a temperature of $100^{\circ} \mathrm{F}$., the sheet should be so applied that one part of the body does not come in contact with another. Small pieces of ice are rubbed over the sheet, first in front and then behind. The head should be sponged with cold water and a hot-water bottle applied to the feet during this procedure. After the ice has been rubbed upon the sheet for ten minutes the child is enveloped in the blanket without removing the wet pack. The applications of ice should be made every fifteen or thirty minutes if ordered, and may be continued at these intervals for one or twentyfour hours. .

Graduated Cold Bath.-The child is placed in a tub of water at a temperature of $100^{\circ} \mathrm{F}$. The temperature is then gradually reduced by the addition of ice wrapped in towels or by cold water until the temperature of the water is $85^{\circ} \mathrm{F}$. or $80^{\circ} \mathrm{F}$. While in the tub the child's body should be vigorously rubbed and an ice-bag or cold cloths should be applied to the head. The bath may be continued for ten or fifteen minutes. Upon removal the child's body should be quickly dried and wrapped in a warm blanket. The temperature of the child should be taken half an hour after removal from the bath.

Cold Irrigation of the Colon.-Water of $40^{\circ} \mathrm{F}$. to $50^{\circ} \mathrm{F}$. is injected through a catheter into the rectum. About a pint should be injected at one time. When introducing the catheter, if the end is placed immediately within the sphincter and then a small amount of water allowed to run, the rectum will dilate and permit the further introduction of the catheter to be accomplished easily. 
Measures to reduce temperature should be stopped if the child becomes very blue.

\section{COUNTER-IRRITATION}

Cantharides Blister.-The surface of the skin which is to be blistered is thoroughly scrubbed and washed with alcohol. The cantharides plaster should be cut to the proper size and its surface oiled. It should then be applied and allowed to remain in position for six hours. The plaster should be removed carefully, and if the skin is raised cut the lowest portion of the bleb and allow the fluid to run out, carefully protecting the sound skin. Do not tear the blistered skin, as it causes a great deal of pain. Apply zinc-oxid ointment. If the blister is not raised at the expiration of the six hours apply boric-acid ointment covered with a thick layer of cotton; this will cause it to raise in a few hours.

Mustard Paste or Mustard Plaster.-Take one part of powdered mustard and six parts of wheat flour, mix with white of egg or lukewarm water, and spread between two layers of old linen or muslin. White of egg is used in preference to water, as it prevents blistering.

In pulmonary diseases the mustard paste should surround the chest, and in heart failure it should cover the entire trunk.

Mustard Poultice.-One part of mustard added to six parts of flaxseed and thoroughly mixed in lukewarm water. When ordered the strength can be increased up to one part of mustard to three parts of flaxseed. It is a very useful method of applying mustard over longer periods.

Mustard Bath (Heubner's).-An extremely heroic mustard bath given in some cases of collapsed lung. 
The proportions are one pound of mustard to one and a half quarts of water. A sheet is wrung out in the above and wrapped about the child from the axillæ to the feet; it is allowed to remain in this dressing for from fifteen to thirty minutes. The mouth, nose, and eyes must be protected from the mustard fumes. After removing the sheet the child must be given a warm tub in order to thoroughly remove all traces of the mustard. The real object of this procedure is to get the child to cry vigorously from the irritation of the mustard, and in this way to expand the collapsed portion of the lung. (For Mustard Tub, see page 407.)

Mustard Pack.-The child should be stripped and laid upon a blanket; the trunk should be surrounded with a sheet dipped in mustard water. This is prepared by adding one tablespoonful of mustard to a quart of tepid water. After the wet sheet is applied the child should be wrapped in a blanket. It should be removed in from ten to fifteen minutes.

Turpentine Stupe.-A teaspoonful of turpentine is mixed with a pint of boiling water. A flannel cloth is dipped in this mixture and wrung out very tightly in a stupe wringer. It is then applied to the part affected and covered with oiled silk or wax paper. A thick layer of cotton should be placed over all, to retain the heat.

Camphorated oil, amber oil, and olive oil, four parts, mixed with turpentine, one part, are liniments which may be applied to the chest. They are either rubbed in with the hand or applied by means of flannel cloths wet with the preparation.

Dry Cups.-A small medicine glass is selected, the edges of which should be carefully oiled before using. 
One or two drops of alcohol are placed in the glass, it is then manipulated so that the alcohol covers the sides of the vessel with a thin film, care being taken that no drops of the liquid are present. The alcohol is then lighted, and, while burning, the glass is inverted and the mouth is held firmly against the skin. The skin will be sucked up into the glass on account of the vacuum, and the cup will be firmly held in place. They are allowed to remain in position for fifteen or thirty minutes. To remove, press the skin down at one side of the glass in such a way that air is allowed to enter. The greatest care is necessary in the application of cups to prevent burning of the skin. This is caused by a drop of burning alcohol running down the sides of the glass and falling on the skin, or by overheating the edges of the glass.

Wet Cups.-The skin should be scrubbed. The physician will then scarify the part to be cupped and apply the cups in the same way as described above.

\section{POULTICES}

Antiseptic Poultices.-Absorbent cotton wet with a solution of bichlorid of mercury, I to 4000 ; potassium permanganate, I to 4000 ; or carbolic solution, I to 40 , and covered with green protective or wax paper.

Charcoal poultice is used as a deodorizer. One part of powdered charcoal is added to two parts of linseed and prepared like a flaxseed poultice. Powdered charcoal is spread over the surface.

Digitalis Poultice.-Make a flat bag and fill with leaves of digitalis. The bag should be steeped in boiling water and applied, or soak digitalis leaves, 2 oz. to a pint, in warm water, until they are soft, drain off the 
water, and boil them. The decoction can then be added to flaxseed.

Flaxseed Poultice.-According to the size of the poultice which is required, a quantity of flaxseed is added to boiling water until the mixture is thick enough to stir. It is then thoroughly beaten over a flame to fill the poultice with air, this makes the poultice light. A layer half an inch thick is spread over old linen, applied to the affected part, and covered with wax paper. In some conditions, such as pneumonia and peritonitis, the lightest possible poultices are necessary; layers less than half an inch are then applied.

Spice Poultice.-Equal parts of allspice, cinnamon, ginger, and cloves are placed between two layers of flannel or gauze, which are then quilted. It is then wrung out of hot whiskey or brandy, applied to the part affected, and covered with wax paper. In heating whiskey or brandy they should never be placed upon the stove or near a flame. They should be poured into a cup which is then placed in a receptacle containing boiling water.

Starch Poultice.-The starch is first mixed with a little cold water, and then enough boiling water is added to make a paste. It is spread on old linen or muslin, covered with a layer of gauze, and applied like other poultices. A few drops of laudanum may be sprinkled over the surface of the poultice before applying, if there is much pain.

A preferable form of starch poultice for skin diseases is to make a flat bag and fill with dry potato starch; then dip in boiling water and allow to cool.

Technic.-All poultices should be beaten until they are thoroughly filled with air; this renders them light in 
weight. They should be covered with wax paper or oiled silk and a thick layer of cotton to keep them warm. Newspapers may be used in emergencies. A margin of $I$ inch of the linen should be left to turn in, the surface of the poultice should be anointed with oil to protect the skin, and one poultice should not be removed until another is ready for application, the skin being wiped dry before the new one is put in place. Before applying a poultice, test its temperature by holding it to your face. Do not leave one poultice on over an hour.

\section{COMPRESSES}

Cold compresses are made by wringing cloths out of cold water and applying to the body.

For the eyes small disks are cut from muslin or lint and placed upon a cake of ice. When they are thoroughly cold they are laid over the closed eyelids. They should be changed constantly.

Hot compresses for the eyes are prepared in the same manner, boiling water being used instead of ice. The disk should be tested upon the back of the hand before application.

\section{HOT APPLICATIONS}

Hot-water bags are filled with water as hot as can be borne. All the air should be expressed before screwing on the top, and the bag should be placed in a properly fitting flannel cover to prevent burning of the skin.

Hot Foot-baths.-The bed should be protected with a rubber mackintosh, which in turn is covered with a towel. A small foot-tub is placed upon this, containing enough water to cover the child's feet at a temperature of $\mathrm{II} 5^{\circ} \mathrm{F}$. The exposed portions of the child's legs should be covered 26 
with towels. After three minutes a quart of hotter water is added, care being taken not to allow it to come in contact with the child's extremities. The addition of hotter water is continued at these intervals until the water is as hat as can be borne by the hand. The child's feet should remain in the water at this temperature for about fifteen minutes, when they are quickly dried and wrapped in a warm blanket.

Hot Bath.-The child is placed in a tub of water at a temperature of $100^{\circ} \mathrm{F}$. The temperature is gradually raised to $110^{\circ} \mathrm{F}$. by the addition of hot water. A thermometer should be used so that the temperature of the water is not raised too high. The body should be vigorously rubbed and an ice-cap or cold cloths should be applied to the head while the child is in the bath. The bath should last for ten to fifteen minutes.

Hot Pack.-The clothes are removed and the child is wrapped in a blanket wrung out of water at a temperature of $110^{\circ} \mathrm{F}$. The child should then be rolled in a second dry blanket covering the first. Hot-water bottles should surround the child and an ice-cap or cold cloths should be applied to the head. These hot applications can be applied every twenty or thirty minutes until free perspiration is produced. Hot water or lemonade may be given to induce sweating; the sweat may be continued as long as desirable. At the expiration of the necessary time the moist blanket is removed, care being taken not to expose the child, a warm, dry blanket taking its place. The child is then sponged with warm water and alcohol to remove the perspiration. The undershirt and nightgown are then replaced and the ice-cap removed, the child always remaining between warm blankets. 
Modified Hot Pack.-The child is wrapped in dry hot blankets. Hot-water bottles are placed under the arms, knees, and feet. An ice-cap over the head. Hot lemonade should be given and the pack terminate as above.

Vapor Bath.-The bed should be covered with a mackintosh and blanket. The clothing is removed and a

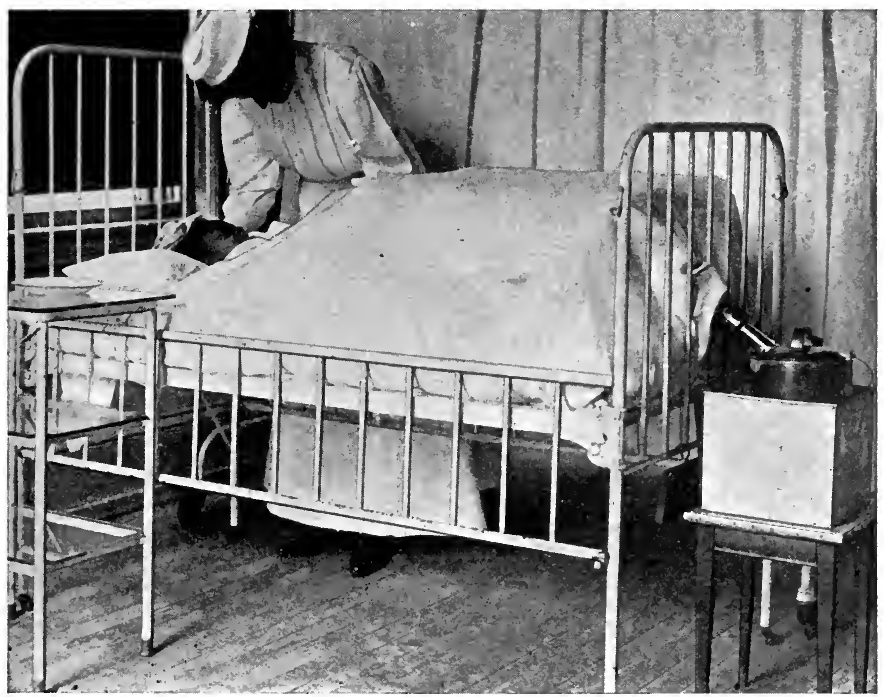

Fig. 96.-Vapor bath. The covers are held above patient by means of a frame. They are tightly tucked in all around bed. The steam is introduced from kettle at foot of bed. An ice-cap is applied to head. Warm or cold beverages are administered to induce perspiration.

blanket is placed loosely over the child. A frame reaching from the neck to well below the feet is placed over the patient. All metal parts of the frame must be covered by a bandage or old muslin to prevent condensation of the 
steam. Over the frame, in the following order, are placed, first, a blanket, then a mackintosh with the rubber side toward the patient, completely covering the frame, and, finally, several blankets covering the whole apparatus. A thermometer should be placed on the chest of the child where it can be readily obtained. The covers are then tucked in securely at the top and both sides of the frame. If desired the blanket which loosely covers the child can now be withdrawn from the opening at the foot. This blanket is usually allowed to remain in giving vapor baths to children. 'The covers are then tucked in about the foot of frame, a small opening being left which should be of sufficient size to allow the introduction of the spout or hose leading from the steam kettle.

Before applying the steam an ice-cap is placed on the child's head, boiling water is placed in the kettle, and the alcohol lamp beneath is lighted. When steam appears, the spout or hose is placed through the opening left for that purpose, care being taken not to place the spout over the child's feet, as the drip from its end is liable to scald the skin.

The thermometer should be read every five minutes, care being exercised not to expose the chest. When the thermometer reads $I 20^{\circ} \mathrm{F}$. the kettle should be removed and the child allowed to remain exactly as before for twenty or thirty minutes. If the thermometer at no time registers $120^{\circ} \mathrm{F}$. the steam is kept up for thirty minutes.

During the bath give hot or cold water freely; hot lemonade is also used at times to induce perspiration.

At the end of twenty or thirty minutes after the thermometer has reached $120^{\circ} \mathrm{F}$. the frame is carefully 
removed from under its covering in such a way that there is no exposure nor disturbance of the covering, the steam thus being retained. The child remains in this position for twenty minutes. At the expiration of this time the moist blankets and mackintosh are carefully removed, warm, dry blankets taking their place. The child is then sponged with warm water and alcohol to remove the perspiration. The undershirt and night-gown may then be replaced and the ice-cap removed. The child should always remain between warm, dry blankets.

In private houses an attachment at times can be made to the steam-heat radiators; this saves a great deal of trouble in chronic cases.

A tea kettle with a garden hose attached to its spout, the other end placed at the foot of the bed, will answer very well when no special apparatus is at hand.

A piece of stove-pipe covered with asbestos can be fitted up to answer the same purpose. Holes should be punched through its sides for ventilation, and it should have an "elbow," so that it can be directed over the foot of the bed. Inside of the stove-pipe, standing on a piece of asbestos, place an alcohol lamp over which can be placed a tin cup filled with water.

In the country or where it is impossible to generate steam by any of the above methods, hot bricks plunged into a basin half-filled with cold water may answer.

Hot-air Bath.- The child and bed are prepared as in the vapor bath, only the steam is omitted. Hot air is introduced beneath the blanket by placing the alcohol lamp beneath the spout of an open kettle. This generates heat at this point, which passes into the bed. 
The stove-pipe, as described above, can be used here, the cup of water being unnecessary.

In many modern houses the electric light can be taken advantage of to give hot-air baths. A 30-candlepower electric bulb should be purchased. The child and bed can be prepared as in the vapor bath, except for an opening, which should be left over the upper portion of frame, through which the bulb can be passed and securely tied to the frame in such a way that there will be no danger of burning the child or the blankets. After the bulb is in place the opening can be closed. All the adjunct measures as described under the vapor bath should be carried out.

A sitting hot-air bath can be given by placing the child wrapped in a blanket on a chair, with his feet in hot water. Blankets draped from his shoulders should cover the child and the chair. Beneath the chair place an alcohol lamp in a bucket, or the 30-candlepower lamp may be used.

In any of the above baths, if pilocarpin has been ordered by the physician, it should be given at the time the nurse is about to begin the preparation for the bath. Then it will be fully active during the bath.

Sitz Bath.-Useful when there is retention of urine, pain in pelvic region, or rectal congestion. A simple method is to place the child in the sitting posture in a tub of water at $\operatorname{II} 5^{\circ}$ to $\operatorname{I} 20^{\circ} \mathrm{F}$. The water should come to the level of the umbilicus.

Salt Baths.-Prepared by adding from 3 to 5 oz. of sea salt to a gallon of water at a temperature of $90^{\circ} \mathrm{F}$. to $100^{\circ} \mathrm{F}$, the number of gallons used in a 
bath varying. When the solution of salt is of sufficient strength the water causes the skin to glow. The baths are useful in rickets.

Mustard Bath.-The bath is prepared by placing four or five tablespoonfuls of dry mustard in a gauze bag, which is shaken in four or five gallons of water until it is thoroughly saturated with the mustard. The water should be at a temperature of $105^{\circ} \mathrm{F}$. when the child is put into the tub, after which it should be slowly raised to I $10^{\circ} \mathrm{F}$. by the addition of hot water. An ice-cap or cold cloths should be applied to the head and the body vigorously rubbed while the child is in the tub. It may be continued for ten minutes, at the expiration of which time the child should be quickly removed and wrapped in a blanket without drying.

Mustard Foot-bath.-At times it is impracticable to put children in a tub; then a mustard foot-bath is useful. The bed is protected by a rubber blanket covered with towels. Cloths are wrung out of mustard water made by adding a teaspoonful of mustard to a quart of water and heated to $110^{\circ} \mathrm{F}$. These are wrapped about the child's feet, the rest of the body being covered. They are applied until the skin becomes red.

\section{BATHS USED IN TREATING SKIN DISEASES}

Bran Baths.-Place one quart of ordinary wheat bran in a gauze bag and place in four or five gallons of water. The bag should be shaken and squeezed until the water resembles a thin porridge. The temperature should be maintained at $95^{\circ} \mathrm{F}$.

Alkaline Baths.-The quantity of water should be 
twenty-two and a half gallons, at a temperature of $95^{\circ} \mathrm{F}$. In this is placed:

$\begin{array}{ll}\text { Carbonate of soda, } & 3 \mathrm{oz} . \\ \text { Bicarbonate of soda, } & 3 \mathrm{oz} . \\ \text { Carbonate of potassium, } & 3 \mathrm{oz} . \\ \text { Borax, } & 3 \mathrm{oz} .\end{array}$

Compound Glycerin Bath.-Water, twenty-two and a half gallons, at a temperature of $95^{\circ} \mathrm{F}$. Ingredients:

Glycerin, 2 oz.

Tragacanth, I oz.

The bath must be used immediately, as this mixture forms glue.

Compound Sulphur Bath.-Water, twenty-two and a half gallons, at a temperature of $95^{\circ} \mathrm{F}$. Ingredients:

$$
\begin{array}{ll}
\text { Precipitated sulphur, } & \text { I lb. } \\
\text { Sodium hyposulphite, } & \text { I oz. } \\
\text { Acid sulphuric (strong), } & \text { I dr. } \\
\text { Water, } & \text { I pt. }
\end{array}
$$

To be mixed and then added to tub.

Linseed Bath.-Add one pound of linseed to twentytwo and a half gallons of water at a temperature of $95^{\circ} \mathrm{F}$.

Starch Bath.- Take four tablespoonfuls of crushed starch and make a paste by adding cold water. Then add two quarts of boiling water, stirring over a fire until it makes an ordinary laundry starch. 'To twenty gallons of water at a temperature of $95^{\circ} \mathrm{F}$. add $4 \mathrm{oz}$. of washing soda and then add the cooked starch.

If especially ordered, $4 \mathrm{oz}$. of glycerin and $4 \mathrm{Oz}$. of borax may be added to the above, or the following: 
Two to four teaspoonfuls of this mixture are added for every gallon of water, with double the amount of dry starch.

Tar Bath.- The patient is rubbed with oil of cade on the diseased patches and then is given a warm bath or a plain starch bath.

Vinegar-and-Mercury Baths.-Water, twenty-two and a half gallons, at a temperature of $95^{\circ} \mathrm{F}$. Ingredients:

$\begin{array}{ll}\text { Vinegar, } & \text { I pt. } \\ \text { Glycerin, } & \text { I pt. } \\ \text { Bichlorid of mercury, I dr. }\end{array}$

\section{HYGIENIC BATHS}

Tepid Bath.-Given at a temperature of from $95^{\circ}$ to $100^{\circ} \mathrm{F}$. It is useful in nervous conditions and to induce sleep.

Shower Baths or Sponge Baths.-The child should stand in a foot-tub containing warm water. A large sponge holding about a pint of water at from $40^{\circ}$ to $60^{\circ} \mathrm{F}$. should be squeezed three or four times over the chest, shoulders, and spine of the child, the skin being vigorously rubbed meanwhile. The bath should not last more than half a minute, and should be followed by a brisk rubbing until a thorough reaction is established.

\section{SYRINGING}

Eye Syringing.-The lids should be massaged to remove pus, then held apart by the fingers, and any discharge dislodged from beneath them. A soft-rubber ear syringe is filled with saturated solution of boric acid heated to $100^{\circ} \mathrm{F}$, and the nozzle is placed at the inner canthus of the eye. The solution should be wiped away 
with antiseptic cotton. Always wipe toward the external canthus, to avoid contamination of the other eye. Medicine which is to remain in the eye is dropped in at the external canthus. The rubber ear syringe is safer to use

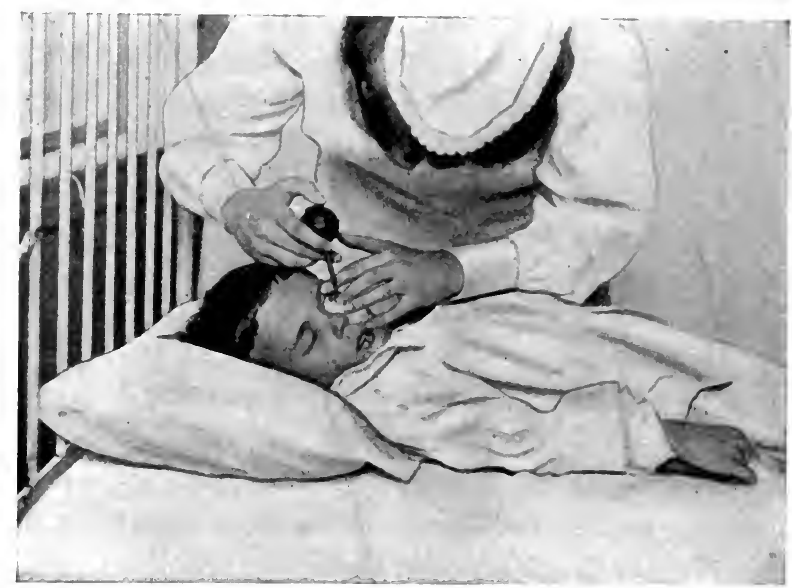

FiG. 97.-Method of syringing eye. The cotton held against the nose should prevent any infection of other eye.

than the ordinary glass eye-dropper, as children are prone to struggle.

Ear Syringing.-An ear syringe is filled with water at a temperature of $110^{\circ} \mathrm{F}$. The soft-rubber nozzle is placed within the external auditory canal and the bulb gently squeezed. A half pint to a quart of water is used.

A fountain syringe held on a level with the ear can be substituted for the small syringe. The bag should be filled with a quart of water at $110^{\circ} \mathrm{F}$., and a small nozzle held in the auditory canal. Do not raise bag above level of ear as it causes too great a pressure. 
Nasal Syringing.-A soft-rubber nasal syringe is filled with an antiseptic solution. The same syringe should

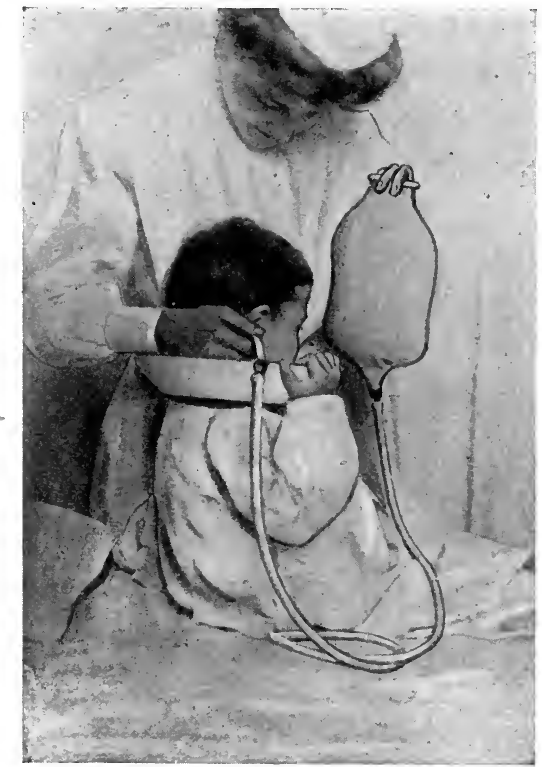

F 1G. 98.-Method for syringing ear with fountain syringe. The lower end of bag should not be above level of auditory canal.

not be used for more than one child unless thoroughly disinfected.

Two positions may be used in nasal syringing. In diphtheria, scarlet fever, or any severe illness the child should not be removed from the bed. In such cases the head should be held on one side, the syringe being placed in the upper nostril. Then the child's head should be 
turned to the other side and the other nostril syringed. The alternate syringing should be continued until the nose is clean. When syringing, the water should run out of the opposite nostril or out of the mouth.

The other method is to hold the child erect on the lap with the head inclined a little forward, the syringing being done by a person who stands behind.

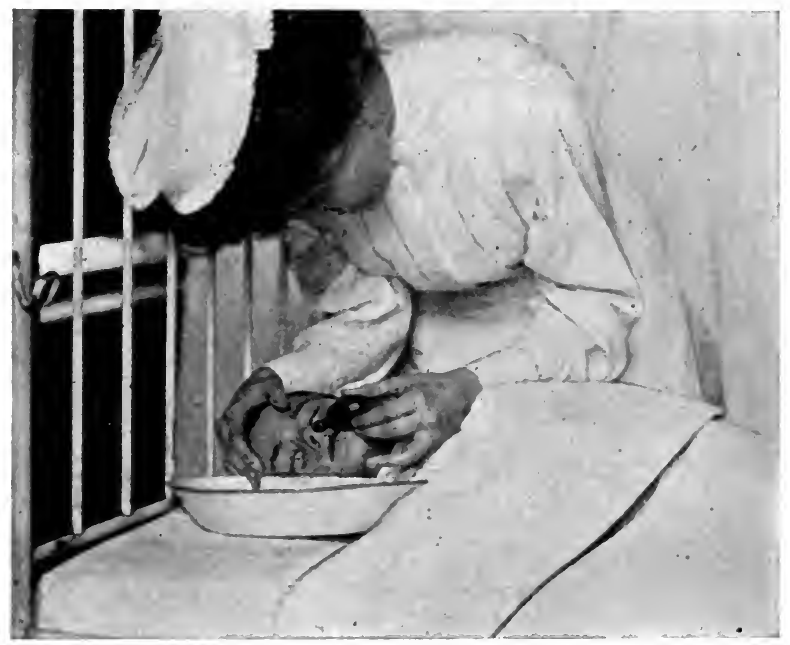

Fis. 99.- Method for syringing nose. The syringe is introduced into upper nostril, the solution escaping from opposite nostril or mouth.

Just as small an amount of pressure should be exerted when syringing the nose as possible, on account of the danger of forcing the infection into the Eustachian tube and causing an otitis media.

At times a fountain syringe is used to irrigate the nose. The bottom of the bag should not be orer two feet above the child's head. 
Syringing of the Mouth and Pharynx.-A Davidson syringe may be used. If the pharynx is to be reached the nozzle is used as a tongue depressor. This should be placed at the angle of the mouth between the back teeth. The child should be held in the sitting posture, with the head inclined forward.

\section{INHALATIONS}

Croup Tent.-A croup tent is made by placing a blanket over a frame in such a way that the entire bed is

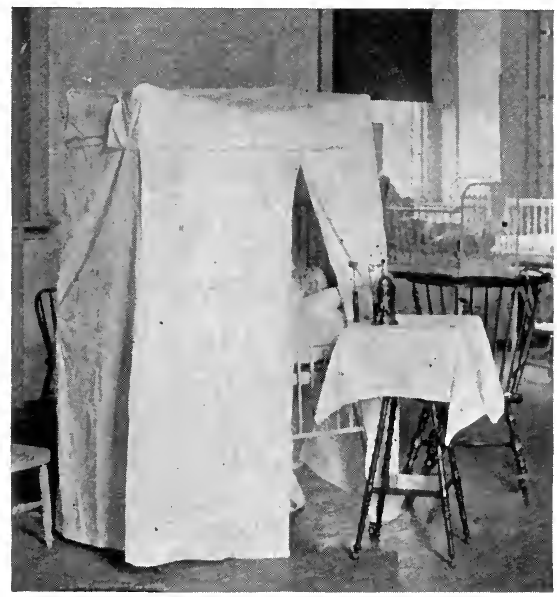

Fig. 1oo.-Croup tent (J. P. C. Griffith).

covered except for a small aperture at the side of the bed near the head which is required for ventilation. Blankets are used instead of sheets, as the latter are liable to catch fire. If a regular frame is not available, a good substitute can be made by erecting broom-sticks at the 
four corners of the bed and stretching a cord around the tops of the sticks. A very good tent can be improvised by throwing a large blanket over an umbrella.

A croup kettle heated by a safety alcohol lamp should be placed upon the floor or on a low box beside the crib,

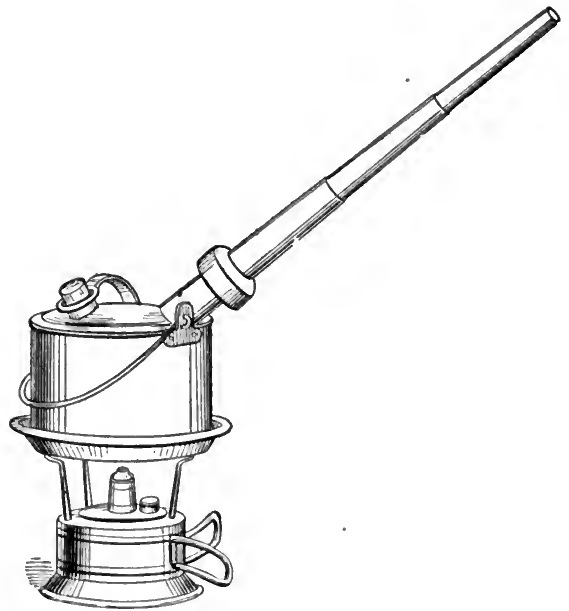

Fig. 101.-Croup kettle (J. P. C. Griffith).

so that the end of the spout is just inside the tent at a level of the surface of the bed.

The kettle is filled with boiling water and a dram of the compound tincture of benzoin may be added. The medicated steam vapor is very soothing in inflammations of the respiratory tract. Great care must be taken to prevent the tent or bed-clothes from catching fire.

\section{STOMACH WASHING OR LAVAGE}

A soft rubber catheter, size I6, American scale (24 French), with a large eye, is attached to rubber tubing 
by a glass joint. A funnel holding from 4 to $6 \mathrm{oz}$. is inserted in the end of the tube. The child should be held in a sitting posture, the body protected by a rubber sheet and the catheter moistened. While the tongue is depressed with the forefinger of the left hand the catheter is rapidly passed into the pharynx and down the esophagus. About ten inches of the catheter should be passed beyond the lips. When it has reached the stomach the funnel is

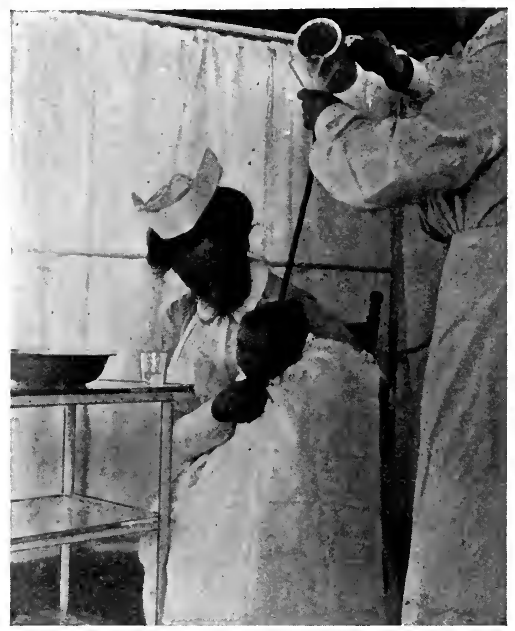

Fig. 102.-Lavage.

raised higher than the level of the infant's stomach and from 4 to $6 \mathrm{oz}$. of water poured into it from a pitcher. When this has run into the stomach the funnel is lowered and raised three or four times to remove any stomach contents, and then lowered below the level of the infant's stomach, which siphons out the water and stomach contents. This should be repeated until water runs clear.

In older children the funnel should be refilled several 
times before siphoning out the contents, as the capacity of the stomach is greater. The water should be boiled and be at a temperature of $I I 0^{\circ} \mathrm{F}$. when used. When

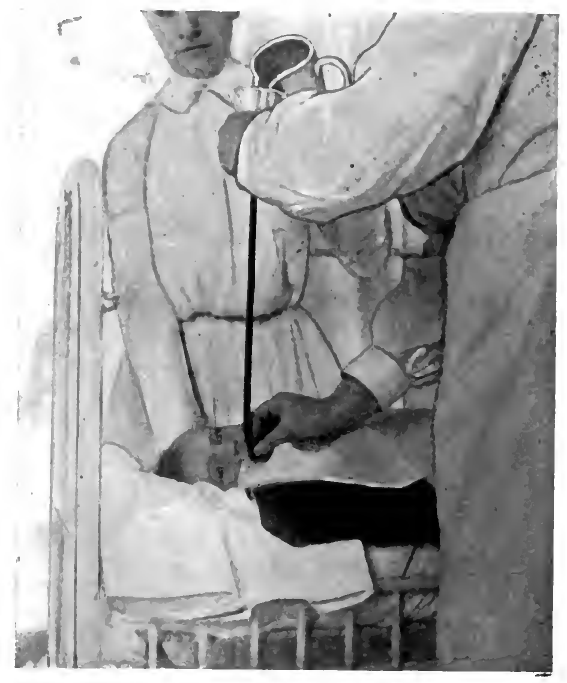

FIG. 103.-Gavage.

the siphoned water runs clear remove the catheter from the stomach. To siphon successfully there must be some water remaining in the funnel when it is lowered.

Care must be taken in giving both lavage and gavage that the child does not bite off and swallow the tube, for if such an accident happens there is nothing to do but open the stomach.

\section{GAVAGE (FEEDING BY STOMACH-TUBE)}

Gavage (Feeding by Stomach-tube).-'The same apparatus is used as in stomach washing. The child should be wrapped in a blanket. Sometimes, where there is great resistance to the introduction through the mouth, it may 
be passed through the nose. In older children a mouth gag is often necessary. A good substitute is a large spool, the catheter being passed through the hole in the spool. After the tube has entered the stomach the funnel should be raised to allow the gas to escape. The food is then poured into the funnel; as soon as it has disappeared the tube is tightly pinched and quickly withdrawn to prevent food from trickling into the pharynx, which often causes vomiting. In young infants, after removing the tube, it is well to keep the jaws open for a few moments to prevent gagging.

Food given by gavage is often predigested; the intervals between feedings must be longer than under other circumstances, and at times the stomach should be washed first.

\section{IRRIGATION OF THE COLON}

The child is placed upon its back, brought to the edge of the bed with the thighs flexed, and the buttocks slightly elevated. A soft rubber catheter is attached to an ordinary fountain syringe, the bag containing the water being hung 4 or 5 feet above the bed. The water should be at a temperature of $85^{\circ}$ or $90^{\circ} \mathrm{F}$. in ordinary cases; when there is shock normal salt solution at a temperature of $I I^{\circ} \mathrm{F}$. is used. The catheter should be greased before introduction and a small quantity of the water allowed to run off. It should then be placed within the sphincter when the water is allowed to flow. This distends the rectum and allows further insertion to be accomplished easily. The catheter is pushed in slowly to a distance of $\mathrm{I}_{2}$ or $\mathrm{I} 4$ inches. Usually a pint and often a quart will be introduced before any water returns. The irrigation should be continued until the water returns clear; at times a gallon of water is used 
for a single irrigation. Gentle kneading of the abdomen should be continued during the procedure. At the end of the irrigation the rubber tube is detached and the water allowed to escape through the catheter.

\section{CONTINUOUS SALINE INJECTION}

This is used by many surgeons following operations on septic cases, especially appendectomy. It is useful whenever children are greatly depleted.

An ordinary fountain syringe is filled with normal salt solution at a temperature of $115^{\circ}$ to $120^{\circ} \mathrm{F}$. This is tied to the foot of the bed not over a foot above the level of the buttocks. Two hot-water bags, tied together, are suspended over the bag containing the salt solution, one on either side, and a blanket is wrapped around them. The hot-water bags can be refilled from time to time as they cool. The tube is then carried under the bed-covers and over hot-water bags, which lie on the bed, to the child's buttocks, which are elevated. Here it is attached to a specially prepared rectal tube (Murphy's tube). A catheter can be used in place of a Murphy tube if the latter is not at hand. The catheter should be inserted from four to six inches in the rectum. If the tube is expelled it must be strapped in with adhesive plaster.

The flow of the salt solution is controlled by a stop-cock or, better, a hemostat. The solution should drip (a drop at a time) into the rectum. By shutting off one-half or more of the caliber of the tube by means of the hemostat this can be regulated.

The idea of giving the solution so slowly is to have it all absorbed. Usually, however, there is leakage, and the clothes must be changed frequently and the bed protected.

The system of hot-water bottles will keep the solution 
at the proper temperature. When the solution is not retained a good plan is to give it for two hours, then discontinue for the same length of time.

\section{ENEMATA}

An enema consists in the injection of soapy water into the rectum. The water should be at a temperature of $85^{\circ}$ or $90^{\circ} \mathrm{F}$

High Enema.-A catheter should be attached to the nozzle of the fountain syringe and thoroughly greased. A small amount of water should be allowed to run off before introduction; then place the catheter within the sphincter and start the flow; this allows it to be pushed in further without doubling up. Where an immediate effect is desired the most efficient enema contains one teaspoonful of glycerin. Oil enemas are useful where the fecal mass is hard and dry and expelled with difficulty.

Low enemas are given in the same manner; the water is injected by the introduction of the nozzle of syringe within sphincter, the catheter being unnecessary.

Nutrient enemata are sometimes used. They should be peptonized.

When drugs are given by enemata milk is sometimes used as the fluid.

\section{HYPODERMICS}

The skin should be rubbed with alcohol and then pinched between the thumb and forefinger, and the needle plunged firmly into the subcutaneous tissue. Veins must be avoided. The solution is injected slowly. After the withdrawal of the needle the part should be kneaded with the fingers. If the drugs used are of an irritating nature hot sponges of cotton should be applied to the part. The hypodermic needle must be sterile. 
For the administration of gelatin solutions sterile "horse hypodermics" are used. The preparations must be injected slowly and the punctured wound covered with collodion. Antitoxin is sold in sterile hypodermic tubes. The method of introduction is the same.

\section{BACTERINES AND VACCINES}

These are emulsions of dead bacteria which are injected into the patient to stimulate phagocytosis, based on Wright's opsonic theory.

Phagocytosis is the property of the white blood corpuscles to destroy germs and to eliminate toxins or poisons.

In many conditions it does a great deal of good.

The technic of administration is the same as for any hypodermic injection.

\section{VAGINAL DOUCHING}

A fountain syringe with a catheter attached to the nozzle is used. The catheter should be sterile and greased before introduction into the vagina. 'The solutions used for douching are bichlorid of mercury, I: 10,000; potassium permanganate, I: I0,000; saturated solution of boric acid; and salt solution. They should be at a temperature of $110^{\circ} \mathrm{F}$, and from one to two quarts are used.

\section{VAGINAL INJECTIONS}

Argyrol is the drug most often used. Three drams of a Io or 20 per cent. solution of argyrol are placed in a glass syringe having a sterile rubber catheter attached to the nozzle. The parts are thoroughly cleaned and the solution injected. The catheter is quickly withdrawn and the vulva is held together for several minutes, when the solution is allowed to run out. Argyrol deeply 
stains all linens. It is sometimes administered in the form of vaginal suppositories.

\section{ARTIFICIAL RESPIRATION}

Place the child on its back on a flat surface with a blanket rolled (not folded) under the shoulders and neck in such a way to allow the head to fall backward enough to straighten the windpipe or trachea. Open the mouth, forcing the jaw if necessary. If the jaw is rigid it can be forced open by placing the forefinger back of the angle of the lower jawbone and the thumbs of both hands on the chin, pulling forward with the fingers and pressing the jaw open with the thumbs. Place something between the teeth to keep the jaws open and to prevent the child from biting its tongue, using something large enough to remove all danger of it being swallowed accidentally. Grasp the tongue with a hemostat, having an assistant hold it out. In the absence of a hemostat or tongue-

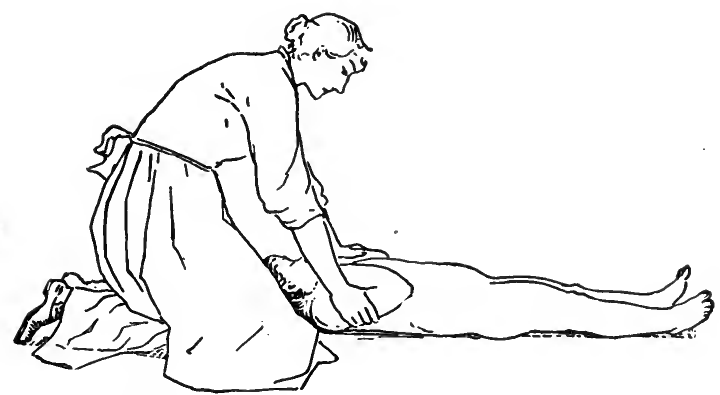

FIG. 104.-Resuscitation after drowning: first movement (J. P. C. Griffith).

forceps the tongue may be grasped between the index and second finger, covered with a handkerchief. Clear the mouth of mucus by inserting the forefinger as far 
as possible and bringing up the froth with a scooping motion.

Sylvester's Method.--Stand or kneel behind the head of the child. Bend its arms so that the hands meet on the chest, grasp the child's forearms firmly, as close as possible to the elbows. I. Firmly press the child's elbows against the sides of the body, so as to force the air out of the lungs. 2. Raise the arms slowly, with a sweeping motion, until the child's hands meet above (or behind) its head. 3. While the arms are extended in a line with the body, give them a slow, strong pull, until the chest is fully expanded. 4. Bring the arms, with bent elbows, down against the sides and press them firmly, as before.

This action should be continued about fifteen times a minute until the child begins to breathe. Care must be exercised against a tendency to make these motions too fast; they must be done slowly. A good plan is to count

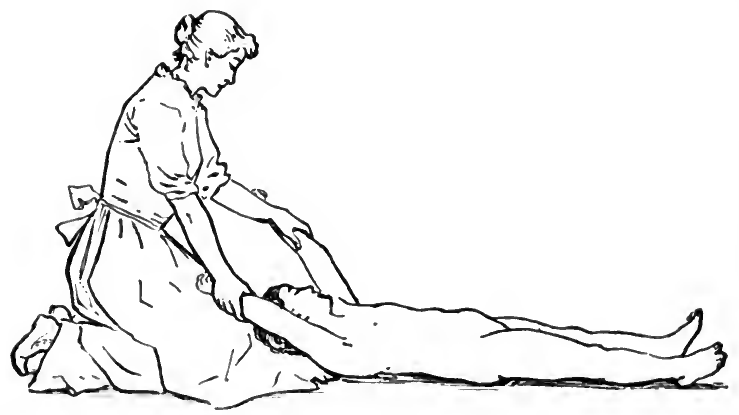

FIG 105.-Resuscitation after drowning: second movement (J. P. C. Griffith).

four slowly - "one," as the pressure is made on the sides of the chest; "two," as the arms are being extended above the head; "three," as the strong pull is given; and "four," when the arms are again being bent and returned to the side. 
Do not let the hands on the forearm slip away from the elbows; the best results are obtained with the hands in this position.

The operator must appreciate the fact that this manipulation must be executed with methodic deliberation, just as described, and never hurriedly or half-heartedly. To grasp the arms and move them rapidly up and down like a pump-handle is both absurd and absolutely useless.

Each time the arms are extended above the head the tongue should be drawn outward and downward. The chest should be slapped from time to time with a wet towel, and the extremities vigorously rubbed.

After the child has responded to treatment stimulate it and surround it with hot-water bottles.

In performing artificial respiration, if the child does not show any signs of returning vitality, do not be discouraged, but continue the motion regularly for at least one hour, summoning such assistance as you may need.

\section{ASPHYXIA IN THE NEWBORN}

At times the child fails to breathe after birth. Under such conditions it is necessary to stimulate the respiratory centers. This usually can be accomplished by slapping the child, pouring ether or cold water over the chest, and removing mucus from the mouth; or if these methods fail, by grasping the base of the ribs between the thumb and fingers, the thumb on one side of the body and the remaining fingers on the other; by firmly squeezing the fingers together the air is forced out of the lungs, and upon relaxing the fingers the chest expands, filling the lungs. The base of the ribs should be forced together in this manner at the rate of about thirty times to the minute. 


\section{HYPODERMOCLYSIS}

This is the introduction of normal salt solution under the skin. For this purpose is used a sterile fountain syringe or glass reservoir with a special needle attached to the end of the rubber tube. The needle of a "horse hypodermic" can be used. "The bag is filled with the necessary quantity of normal salt solution, at a temperature of $120^{\circ} \mathrm{F}$., which has been sterilized on three successive days. After the cold water has run off, the needle is plunged through the skin. The pectoral and gluteal regions are usually selected as the places for injections. When the needle is in place the normal salt solution is allowed to run slowly, and continued until the amount ordered, varying from I to $8 \mathrm{oz}$., has entered the subcutancous tissues. A small collodion dressing is applied to the puncture. Use a sterile thermometer for taking temperature of salt solution.

\section{INTRAVENOUS INJECTIONS}

This consists of the introduction of normal salt solution into a vein. The physician usually selects a vein at the inner side of the elbow-joint. A bandage is tied tightly around the arm above the joint to engorge the vein. $\mathrm{He}$ dissects the vein away from the surrounding tissue and places a grooved director beneath it. A ligature of catgut is thrown around the vein, below the point of inserting the needle, and tied. A second ligature is placed in position above the point of insertion, but it is not tied until after the injection has been given. A sterile fountain syringe or glass reservoir is filled with the required amount (usually a quart) of normal salt solution, which has been sterilized on three successive days, and a hypodermoclysis 
or horse hypodermic needle attached to the end of the rubber tube. The normal salt solution should be at a temperature of $110^{\circ}$ to $120^{\circ} \mathrm{F}$. (use sterile thermometer). When the physician is ready to introduce the needle into the vein the solution is allowed to run; it should be running when it is introduced into the vein, as this avoids the entrance of air into the vessels, which is a very dangerous accident. The bandage should be cut as soon as the needle enters the vein. When the solution has run into the vein the upper ligature is tied before the needle is removed. The skin wound is then stitched and an aseptic dressing applied.

The instruments needed in this operation are a scalpel, forceps, hemostats, grooved director, ligatures, and a fountain syringe with proper needle; also roller bandage. The arm should be prepared as for an operation.

\section{EXTENSIONS FOR FRACTURES AND COXALGIA}

A strip of adhesive plaster, 2 inches wide, is cut long enough to extend from the outer portion of the knee or middle of the thigh to a point 2 inches below the sole of the foot and from there to the middle of the thigh or knee on the opposite side of the leg. The adhesive is applied to the outer portion of the leg, as far as the anklejoint. It is not attached to the foot, and 4 inches are allowed for the loop around the foot. It is then carried to the opposite ankle-joint and applied to the inner side of the leg. A bandage starting at the anklejoint is applied to the leg as far as the adhesive strips extend.

A small block of thin wood, 3 inches long and 2 inches wide, is covered with adhesive, a hole bored in the center, and the board placed in the middle of the loop 
below the foot and held in place by a strip of adhesive. Through the perforation in the block a wire is passed, which is firmly attached to the inner side of the block. The wire should run over a pully at the foot of the bed and have a four or five-pound weight attached at the base which should clear the floor by several inches. A wad of cotton is placed beneath the tendo Achillis to prevent pressure at this point. 'The foot of the bed is clevated to obtain counter-extension.

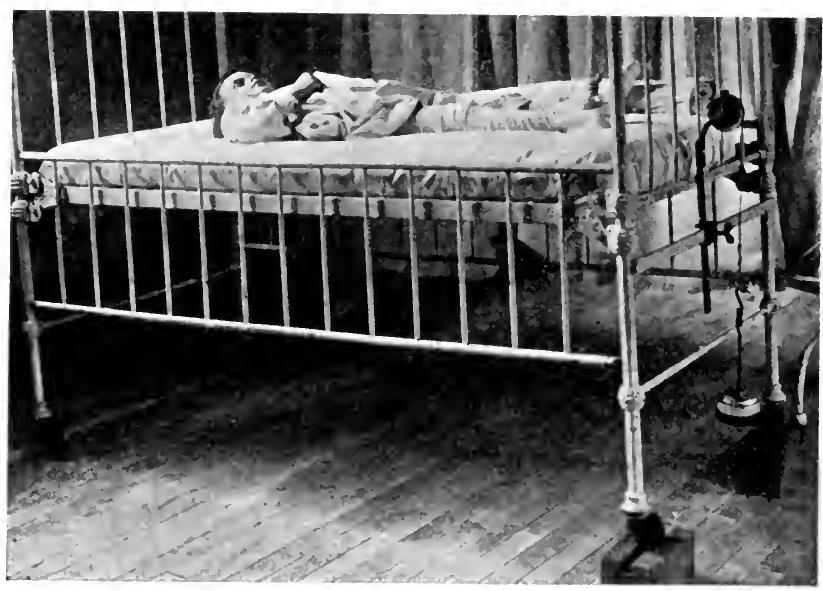

F1G. 106.-Buck's extension apparatus. The foot of the bed is elevated to obtain counterextension.

Care must be taken in the remoral of old adhesive strips that the skin is not pulled off with the plaster. Alcohol or ether will rencler this task easier.

DRESSING FOR FRACTURE OF THE FEMUR IN CHILDREN OVER TWO YEARS OF AGE

Hamilton Splint.--This is the best dressing to apply in childhood. It consists of (I) two long splints; the ex- 
ternal reaches rom the axilla to the sole of the foot and the internal extends from the groin to the sole. They are 4 or 5 inches wide at the hip-joint and taper to 3 inches at the ankle. (2) Two long bags filled with bran, the external reaching from the axilla to the ankle and the internal extending from the groin to the internal malleolus. (3) A Buck's extension apparatus applied as described above. (4) A sand-bag reaching from the axilla to the ankle along the uninjured side.

Method.-A piece of unbleached muslin of sufficient length to reach from the axilla to the sole and a yard wide

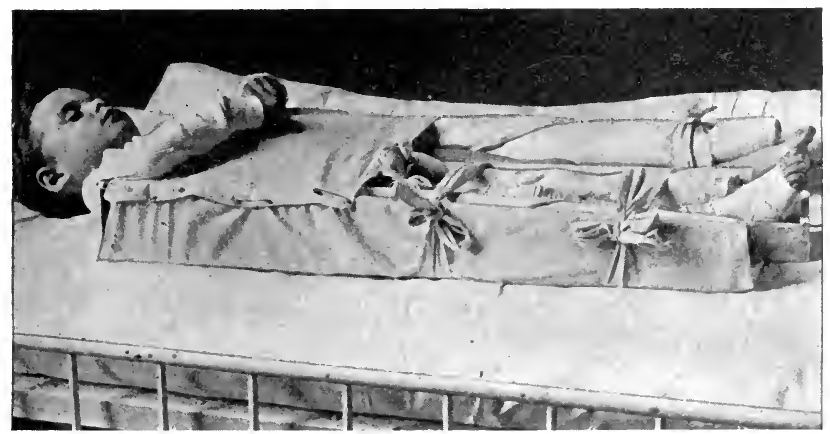

FIG, 107.- Hamilton splint. First apply a Buck's extension. The injured leg is held in position by bran-bags between wooden side-splints, a long sand-bag balances the dressing on the sound side, to which is tied the uninjured ankle. A shot-bag is placed over upper fragment of fractured bone.

is placed beneath the child. At a point corresponding to the level of the groin the muslin is cut through half its width. The extension apparatus is applied and the bran-bags put in their proper places in close apposition to the leg. The splints are then laid on the edge of the muslin and folded in until they fit close to the bran-bags and hold them snugly to the leg. Three or four strips of bandage placed around the dressing keep the splints 
in place. The sand-bag is placed along the uninjured side. The upper portion of the unbleached muslin, which has not been folded in by the internal splint, is then carried around the body, including the sand-bag, and over the external splint, to hold the upper end in position. A weighted shot-bag is placed over the upper fragment of the bone and the foot of the bed is elevated.

The necessity of frequent changing of the dressings and clothing of the child, from contamination with urine and the stools, renders it necessary, at times, to apply a moulded pasteboard splint beneath the fractured thigh, which should extend upward as far as the waist and be firmly held in place. This method of dressing does away with the pain from motion which always attends the process of redressing.

\section{MOLDED PASTEBOARD SPLINTS}

Technic.-The pasteboard is cut in the proper shape and of the proper length, and dipped in hot water. When

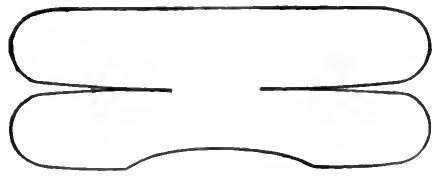

FIG. 108.-Jaw-cup, unfolded. A moulded pasteboard splint (Da Costa).

thoroughly wet it can easily be moulded to the part, which shape it holds when dry. It should be padded with cotton before application.

\section{SPLINTS}

Splints are used to keep the broken fragments of bone in apposition after a fracture.

According to the location of the fractured bone different 
kinds of splints have been devised. The principal forms are as follows: shoulder-cap, for fracture of the upper portion of the humerus; the internal angular and anterior angular splints for fractures of lower end of humerus

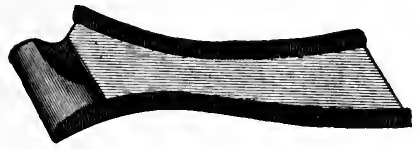

Fig. ro9.-Bond's splint (Da Costa).

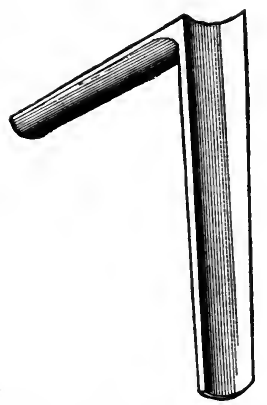

FIG. I I1.--Anterior angular splint (Da Costa).
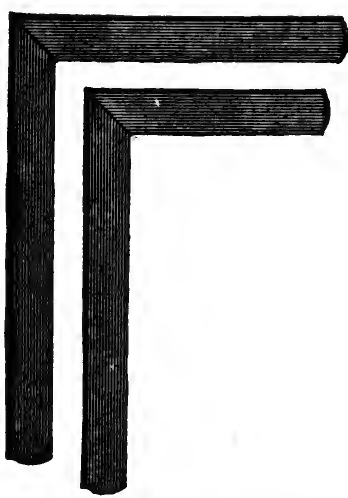

FIG. rro.--Internal angular splint (Da Costa).

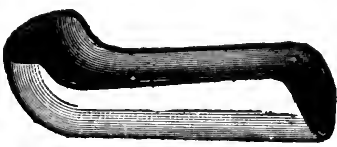

Fig. I $12 .-$ Shoulder-cap (Da Costa).

and upper portion of the bones of the forearm; and the Bond splint for fracture of the lower portion of the bones of the forearm.

\section{FRACTURE-BOX}

This is a special box used for fractures of the lower portion of the leg. It is so constructed that the sides are movable and the foot-piece perforated. 
A pillow is placed upon the bottom of the box, while the sides are down, upon which is rested the fractured leg in such a manner that the foot is held firmly at a right angle against the foot-piece of the box, being secured in this position by a strip of bandage through the perforations. A wad of cotton should be placed beneath the heel and sole of the foot. The sides of the box are then turned

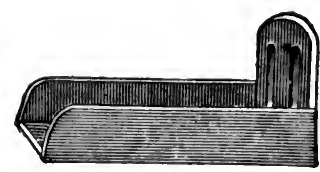

Fig. 113.-Fracture-box (Da Costa)

up and held in the upright position by three or four strips of bandage surrounding the box. This causes the leg to be held firmly between the two sides of the pillow.

\section{AIR-BEDS AND CUSHIONS}

Air-beds are useful at times in injuries to the back. Water-beds are also used.

Air-cushions are very useful in relieving a part from pressurc.

\section{PLASTER CASTS}

Plaster casts are very useful in childhood. They are used for fractures, for keeping joints immobile, and for keeping the bones straight after an ostcotomy. Specially prepared bandages are used, which should be soaked in luke-warm water immediately before application. 


\section{FRAMES}

Frames are used in Pott's disease, in order that the backbone may be kept immobile. The child is placed upon the frame in such a position that the buttocks are situated at the opening in the canvas. This permits bowel movement without removal from frame. The child is held in position by unbleached muslin, which is attached to the two side bars of the frame and cut of sufficient length to extend from the axilla to the base of

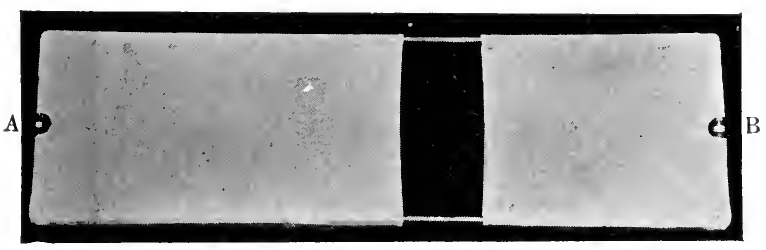

Fig. x14.-Modified Bradford frame. Devised by Dr. Fauntleroy. The daily tightening of the nuts $\mathrm{A}$ and $\mathrm{B}$ keeps apparatus rigid.

the frame. This covering should be tightly drawn across the child and firmly attached to the opposite bar.

The Bradford frame, or some of its modifications, is the best. Fauntleroy's nodification permits of the taking up of the slack by daily tightening the nuts in the upper and lower bars. This saves the trouble of constant tightening of unbleached muslin.

\section{OILED-SILK JACKET}

This is a very good method of obtaining a mild, constant counter-irritation of the chest, formerly used extensively in cases of pneumonia and bronchitis. The jacket is prepared by cutting out three layers, according to the pattern shown on the following page. 
The outer layer is oiled silk, the middle layer is cotton batting, and the inner layer gauze. For a child one year

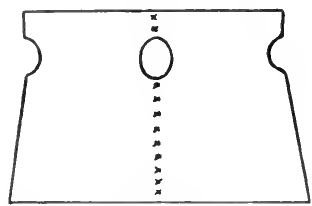

Fic. 115.-Pattern for oiled-silk jacket.

of age the dimensions should be $\mathrm{I} 2$ by I 2 inches. A properly prepared jacket should last about two weeks.

\section{STRAIT-JACKET}

When it is necessary, in very restless children, to control their movements the strait-jacket is of use.

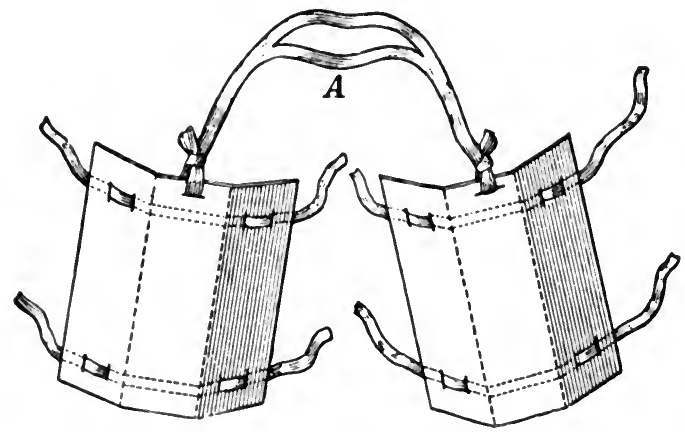

Fig. 116.-Model for the dressing shown in Fig. 117 . The orening in the calico strip $A$ is for the head, so as to prevent slipping of the pasteboard splints (Frühwald and Westcott).

It is made of unbleached muslin, double thickness, a yard wide and cut long enough to reach from one side of the bed to the other. It is attached to the frame of 
the bed on both sides and fastened securely enough to hold the child flat upon its back. Two armholes are cut at the proper level and distance apart and these

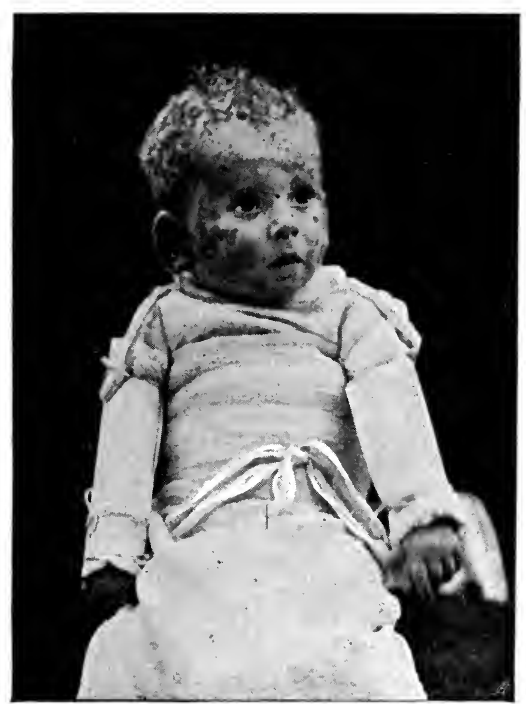

Fig. 117.-Dressing to prevent scratching in eczema of the head (Frïhwald and Westcott).

should be bound. A wad of cotton should protect the skin of the axillæ from chafing.

\section{CUFFS}

Cuffs are necessary in children who have a tendency to pick at their dressings or to scratch irritating lesions of the skin.

Celluloid cuffs can be purchased which should be well padded before being applied. 
Pasteboard cuffs are made by cutting stiff pasteboard of sufficient length to extend from the armpit, or axilla, to the wrist, and wide enough to encircle the arm. They should be well padded with cotton and held in place by a bandage (see Fig. II7). This form of dressing prevents the child from bending the elbow.

\section{MASKS}

Masks are useful in the treatment of skin diseases of the face. They are made so as to cover completely the head and face, small apertures being cut for the eyes, nose, and mouth. It is the only means by which applications to the face can be properly applied.

\section{MASSAGE}

Massage is useful in infancy after attacks of infantile paralysis. The affected limbs should be massaged daily to increase the circulation to the part, so that the unaffected muscles will be under the most favorable conditions for hypertrophying or overdeveloping, upon which depends a fairly good use of the leg.

In childhood, massage is one of the best measures to employ in chronic constipation. It should be practised twice a day, after retiring and in the morning. The proper method of giving massage in these cases is to use only the hand, without grease of any kind, rubbing the abdomen with a circular motion, the idea being to move the abdominal wall over the intestine, and in this way to excite peristalsis.

In older children the same causes for massage exist as in the adult. 


\section{ELECTRICITY}

Electricity has a limited scope in childhood. In paralytic conditions it is useful and should be applied in the same manner as in the adult.

\section{SKIAGRAPHY}

For the purposes of diagnosis, especially of fractures, the Röntgen ray is of the greatest use. Medicinally it is not employed.

\section{DISINFECTION}

All discharges should be immersed in carbolic acid, I : 40; bichlorid of mercury, I : 2000; or chlorinated lime of equal strength, and allowed to stand fifteen minutes. All bed-clothing should be thoroughly boiled for a half hour.

Disinfection of Hands.-Remove all dirt from under and around nails. Nails and hands should be thoroughly scrubbed with soap and hot water. Immerse them in 95 per cent. alcohol for not less than one minute, then plunge the hands in a solution of bichlorid of mercury, I: 2000 , or carbolic solution, I:40, and thoroughly wash them for at least a minute. A clean wound should never be dressed after an infected wound. The hands should be disinfected between each dressing.

Full bichlorid baths, I: 4000, should be taken while nursing contagious cases, and given to the patient before release from quarantine.

Fumigation of the apartments, mattresses, hangings, clothing, etc. is accomplished by thoroughly sealing the room and introducing formalin gas through the keyhole. 


\section{DISEASES OF CHILDREN FOR NURSES}

Disinfection of Excreta.-The stools and urine should be received in a vessel containing a disinfectant. An equal quantity of disinfectant to the size of the excreta should then be added and the whole thoroughly mixed, and allowed to stand for half an hour before emptying into the water-closet hopper. The bed-pan should contain disinfectant when not in use. It should be thoroughly rinsed in warm water before placing it beneath the child, otherwise the disinfectant might burn the buttocks.

\section{DRESSING OF BURNS AND WOUNDS}

The burned or scalded area should be covered with lint saturated with carron oil, which is composed of equal parts of linseed oil and lime-water.

All lacerated and punctured wounds should be thoroughly cleansed with hot water and peroxid of hydrogen. They should be covered with a wet bichlorid or sterile normal salt solution dressing and wax-paper.

\section{PREPARATION FOR OPERATION}

Thoroughly scrub the part with tincture of green soap, shaving first, if necessary; rinse with sterile water and alcohol; then a solution of bichlorid of mercury, I : 2000 . Cover with gauze wrung out of $\mathrm{I}: 4000$ bichlorid of mercury, wax paper, and bandage.

At the time of operation this process is repeated.

Many surgeons use tincture of iodin spray to sterilize the skin. This is usually prepared by adding one part of tincture of iodin to three parts of water. It is sprayed upon the skin by means of an atomizer. The skin thus treated peels off with the dressings. 


\section{CATHETERIZATION}

The hands should be thoroughly scrubbed and disinfected. The external genitals should be scrubbed with tincture of green soap and water; washed with sterile water; then with I: 4000 bichlorid solution; then a second time with sterile water to remove all traces of bichlorid.

Soft rubber catheters should be boiled for ten minutes. English and silk catheters should be immersed in $\mathrm{I}: 20$ carbolic solution for ten minutes; then thoroughly rinsed in sterile water before introduction.

When ready to catheterize, dip the end of the catheter in carbolized oil, $1: 40$. If the catheter touches any part before entering the urethra it must be resterilized. This care is taken to avoid infection of the bladder. When removing catheter it should be pinched to prevent the urine remaining in it from running out. If a glass catheter is used the finger should be placed over the opening.

\section{ASPIRATION OF THE CHEST}

A large needle, or trocar, and cannula is used to pierce the chest-wall. The instrument used is attached by means of a rubber tube to a vacuum pump from which all the air must have been removed before the operation.

The child should be prepared for operation in the usual way, the point of the proposed puncture having been previously determined. The instruments used should be sterilized. A small dressing is placed over the puncture. 


\section{PAQUELIN CAUTERY}

The metal reservoir, containing a sponge, should be about one-third full of benzene. The platinum point to

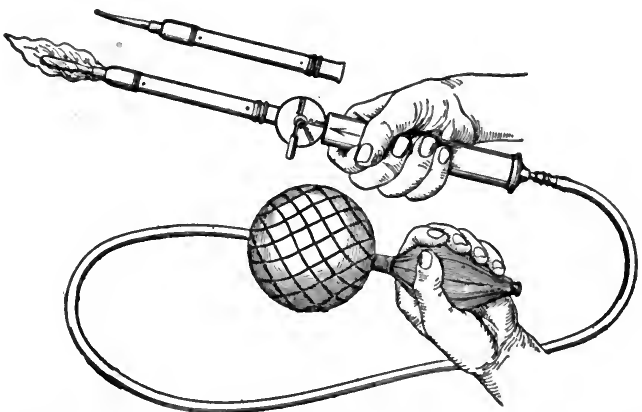

Fig. 118.-Paquelin's cautery. Note that the benzene is contained in the handle of the apparatus (W. E. Ashton).

be used is screwed into position, the tube from the reservoir is slipped over the handle, the point is heated in the lamp, is removed from the flame, and by compressing the bulbs previously connected with the reservoir benzol vapor is forced into the point, which will heat up and can be maintained at any temperature by the rapidity with which the bulb is worked. 


\section{CHAPTER XX}

\section{WEIGHTS AND MEASURES; ABBREVIATIONS}

\section{SCALES OF WEIGHTS AND MEASURES}

\section{APOTHECARIES' WEIGHT}

The pound (libra)

ounce (uncia)

dram (drachma)

scruple (scrupulum)

grain (granum) tb. contains 12 ounces.

3 " 8 drams.

3 " 3 scruples.

Э “ 20 grains.

gr.

WINE MEASURE

The gallon (congius)

pint (octarius)

fluidounce (uncia fluida)

fluidram (drachma fluida)

minim (minimim)
C contains 8 pints.

$\mathrm{O}$ " 16 fluidounces.

$\mathrm{f} \overline{3}$ “ 8 fluidrams.

f3 " 60 minims.

$m$

TABLE OF MUTUAL EQUIVALENTS OF WEIGHTS AND MEASURES

$$
\begin{aligned}
& \text { Hb. } 3 \quad 3 \quad \text { g } \text { gr. } \\
& \mathbf{r}=\mathbf{r}_{2}=96={ }_{2} 88=5760 \\
& \mathbf{I}=8=24=480 \\
& \mathrm{r}=3=60 \\
& \mathrm{I}=20 \\
& \text { C } \mathrm{O} \quad 3 \quad 3 \quad \mathrm{~m} \\
& \mathrm{r}=8=\mathrm{r} 28=\mathrm{r} 224=61,440 \\
& \mathrm{I}=\mathrm{r} 6=\mathrm{r} 28=7,68 \mathrm{o} \\
& \text { I }=8 \text { = } 480 \\
& \mathrm{I}=60
\end{aligned}
$$


In prescription writing the scruple is rarely used at the present time.

\section{THE METRIC SYSTEM}

When the metric system is used, the quantities of liquids, as well as solids, are expressed by weight.

The meter is the unit of length; the gram, of weight; and the liter, of volume.

The prefixes, deca, hecto, kilo, derived from Greek numerals, are used to denote increase, and the prefixes, deci, centi, milli, derived from the Latin numerals, to denote decrease.

$$
\begin{aligned}
\text { I000. } & =\text { I kilometer. } \\
\text { I00. } & =\text { I hectometer. } \\
\text { IO. } & =\text { I decameter. } \\
\text { I. } & =\text { I meter. } \\
.1 & =\text { I decimeter. } \\
.01 & =\text { I centimeter. } \\
.00 \mathrm{I} & =\text { I millimeter. }
\end{aligned}
$$

The cube of a centimeter is called a cubic centimeter,

\begin{tabular}{|c|c|c|c|}
\hline I & meter & $=39 \cdot 39$ & inches. \\
\hline 25 & millimeters & $=1$ & inch. \\
\hline 1 & liter & $=3.3 .8 \mathrm{I}$ & fluidounces, slightly over a quart. \\
\hline I & gram & $=15 \frac{1}{2}$ & grains \\
\hline .065 & “ & $=\mathrm{I}$ & grain. \\
\hline 29.37 & cubic centimeters & $=\mathrm{I}$ & fluidram. \\
\hline 4 & “ & $=15$ & minims. \\
\hline
\end{tabular}
and is written cc., which term is used to denote capacity. It is used almost exclusively. 'Thus, instead of saying I decimeter, we say roo cc., and instead of I deciliter, we say $100 \mathrm{cc}$.

\section{Relation Between the Apothecaries and the Metric}

\section{System.-}


Rule for Converting Troy Weights into Grams.(a) Reduce each quantity to grains, move the decimal point one place to the left, and subtract one-third.

(b) Reduce each quantity to drams and multiply by four.

To Estimate a Dose of a Different Fractional Part of a Grain from the Drug on Hand.-You are often ordered to give a dose of medicine of a different fractional part of a grain from the drug you have. Thus, you may be ordered to give gr. $\frac{1}{40}$ of strychnin when the only solution on hand is one in which ro minims equals gr. $\frac{1}{60}$. To find out how much to give, multiply the denominator of the fraction of the solution on hand by the number of minims in which it is held in solution, and divide the result by the amount ordered. Thus,

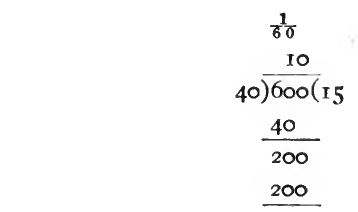

Give 15 drops.

If quantity in a tablet is greater than required.

The given dose is used as the numerator and the required dose as the denominator, thus:

The dose of a tablet is marked $\frac{1}{100} \mathrm{gr}$. , and the required dose is $\frac{1}{150} \mathrm{gr}$.

$$
\frac{100}{150}=\frac{2}{3}
$$

Therefore, two-thirds of the tablet is the required dose. The tablet should be dissolved in fifteen drops of distilled water and two-thirds, or ten drops, administered as the dose. 
To Obtain a Fractional Part of a Minim.-At times $\frac{1}{4}$, $\frac{1}{2}$, or $\frac{3}{4}$ minim may be ordered. 'To obtain the amount correctly, it is necessary to take 5 minims of the drug and add 20 minims of water, making 25 minims in all; then 5 minims of this quantity will represent $\mathrm{I}$ minim of the drug.

If $\frac{3}{4}$ minim is desired, 15 minims of water should be added to the 5 minims representing $\mathrm{I}$ minim of the drug, making 20 minims in all; then 15 minims of this quantity will represent $\frac{3}{4}$ minim of the drug.

If $\frac{1}{4}$ minim is desired, 5 minims of the above will represent the proper amount.

If $\frac{1}{2}$ minim is desired, it is necessary to add 5 minims of water to the original 5 minims representing the drug, making ro minims in all; then 5 minims of this quantity will represent $\frac{1}{2}$ minim of the drug.

In emergencies, 2 drops of a drug can be estimated as representing I minim. By adding 6 drops of water, making 8 drops in all, and then taking 6 drops of this quantity, ${ }_{4}^{3}$ minim may be obtained. Two drops of the above would represent $\frac{1}{4}$ minim of the drug. By adding 2 drops of the water to the 2 drops of the drug and taking 2 drops of this quantity, $\frac{1}{2}$ minim may be obtained. If fractional parts of a drop are ordered, one-half of the above dilutions would represent the proper number of drops to use.

Rule for Making Solutions of Definite Strengths.-

(a) A I per cent. solution contains 5 (4.80) grains of the drug to each ounce of the solution. Therefore, a 2 per cent. solution contains ro grains to the ounce; a 5 per cent. solution, 25 grains; a ro per cent. solution, 50 grains, etc.

(b) A I: 1000 solution contains 8 grains to a pint. 
Therefore, a I: 2000 solution contains 4 grains to a pint, a I:4000 solution 2 grains and a I:8000 solution I grain. The drugs are usually dissolved in water and labeled so many grains to the dram.

\section{DOMESTIC MEASURES}

I teaspoonful $=$ I dram or $4 \mathrm{cc}$.
I dessertspoonful $=2$ drams or $8 \mathrm{cc}$.
I tablespoonful $=4$ drams or I $6 \mathrm{cc}$.
I wine glass $=2$ ounces.
I tea cup $\quad=5$ ounces.
I tumbler

\section{TEMPERATURE}

There are two methods of expressing degrees of heat and cold, Centigrade and Fahrenheit, expressed by the symbols C. and F., respectively.

The zero point of the Centigrade scale is the freezingpoint of water, equal to $32^{\circ} \mathrm{F}$; and the $100^{\circ}$ point Centigrade is the boiling-point of water, equal to $2 \mathrm{I} 2^{\circ} \mathrm{F}$.

Rule for Changing Centigrade Temperatures to Fahrenheit.-

$$
\frac{\mathrm{C} \times 9}{5} \times 3^{2}
$$

Example: 100 C. (boiling-point of water).

C. $100^{\circ} \times 9=900 \div 5=180+32=212^{\circ} \mathrm{F}$.

To change Fahrenheit to Centigrade:

$$
\frac{\mathrm{F}-32 \times 5}{9}=\mathrm{C}
$$

Example : $212^{\circ} \mathrm{F}$. (boiling-point of water).

F. $212^{\circ}-3^{2}=\mathrm{I} 80 \times 5=900 \div 9=100^{\circ} \mathrm{C}$. 


\section{ABBREVIATIONS}

ãā............ of each.

A. c........... before meals.

Liq.......... liquid.

Ad. lib........ as much as desired.

Alt. hor........ every other hour.

Lot.......... . lotion.

Aq............. water.

me..........minim.

Aq. bull....... boiling water.

Mist.........mix.

N......... night.

Aq. dest.......distılled water.

Aq. ferv.......hot water.

No........... number.

Aq. font........ spring water.

Bene..........well.

B. i. d........twice a day.

O............

Ol............ oil.

Ol. oliv....... olive oil.

P. c........... after meals.

Pil........... Pill.

C...........Gallon.

c............ with.

P. r. n....... when necessary.

Pulv.......... powder.

Cc............ cubic centimeter.

Q. 4 H...... every four hours.

Cochl.........spoonful.

Crast.........to-morrow.

D......... dose.

Decub........ lying down.

Q. i. d....... four times a day.

Q. S........... sufficient quantity.

S. F........Whisky.

S. or sig...... directions.

Dil........... dilute.

Dim........... one-half.

S. O. S....... if necessary.

F............ Fahrenheit.

Sine......... without.

Fl. or f....... fluid.

Ft.........make.

Garg.......... gargle.

Gr........ grain.

Gm........... gram.

Gtt.......... drop.

Inf.......... infusion.

Inject........ an injection.

Sp. gr......... Specific gravity.

Spt.......... Spirit.

Ss........... one-half.

Stat.......... immediately.

Syr...........Syrup.

T. i. d......... three times a day.

Tr.......... Tincture.

Troch........ lozenges.

Ung........ ointment.

Lb........... a pound. 


\section{CHAPTER XXI}

\section{MEDICAL TERMINOLOGY}

\begin{tabular}{|c|c|c|}
\hline Prefix & DEFINITION & EXAMPLE \\
\hline $\begin{array}{l}\text { A } \\
\text { dys } \\
\text { end } \\
\text { hemo } \\
\text { hydro } \\
\text { hyper } \\
\text { hypo } \\
\text { macro } \\
\text { micro } \\
\text { peri } \\
\text { pneumo } \\
\text { pyo }\end{array}$ & $\begin{array}{l}\text { absence of } \\
\text { painful } \\
\text { the lining } \\
\text { blood } \\
\text { water } \\
\text { above } \\
\text { beneath } \\
\text { large } \\
\text { small } \\
\text { around } \\
\text { air } \\
\text { pus }\end{array}$ & $\begin{array}{l}\text { asepsis. } \\
\text { dyspepsia. } \\
\text { endocarditis. } \\
\text { hemothorax. } \\
\text { hydrocele. } \\
\text { hyperacidity. } \\
\text { hypodermic. } \\
\text { macroglossia. } \\
\text { microscope. } \\
\text { pericardium. } \\
\text { pneumothorax. } \\
\text { pyogenic. }\end{array}$ \\
\hline SUFFIX & DEFINITION & EXAMPLE \\
\hline $\begin{array}{l}\text { Algia } \\
\text { cele } \\
\text { ectomy } \\
\text { esthesia } \\
\text { gogue } \\
\text { itis } \\
\text { lithiasis } \\
\text { odynia } \\
\text { ology } \\
\text { orrhea } \\
\text { otomy } \\
\text { phagia } \\
\text { pepsia } \\
\text { phonia }\end{array}$ & $\begin{array}{l}\text { pain } \\
\text { a tumor } \\
\text { cutting out } \\
\text { feeling } \\
\text { drugs causing in- } \\
\text { crease of flow } \\
\text { inflammation of } \\
\text { stone in } \\
\text { painful } \\
\text { study of } \\
\text { copious discharge } \\
\text { cutting into } \\
\text { swallowing } \\
\text { digestion } \\
\text { speech }\end{array}$ & $\begin{array}{l}\text { neuralgia } \\
\text { hydrocele. } \\
\text { appendectomy. } \\
\text { hyperesthesia. } \\
\text { cholagogue. } \\
\text { appendicitis. } \\
\text { nephrolithiasis. } \\
\text { pleurodynia. } \\
\text { bacteriology. } \\
\text { diarrhea. } \\
\text { gastrotomy. } \\
\text { dysphagia. } \\
\text { dyspepsia. } \\
\text { aphonia. }\end{array}$ \\
\hline NAME & ROOT WORD & INFLAMMATION OF \\
\hline $\begin{array}{l}\text { Brain } \\
\text { ear } \\
\text { gland } \\
\text { heart } \\
\text { intestine (large) } \\
\text { intestine (small) } \\
\text { kidney }\end{array}$ & $\begin{array}{l}\text { encephalos } \\
\text { ous, otos } \\
\text { aden } \\
\text { cardia } \\
\text { colon } \\
\text { enteron } \\
\text { nephron }\end{array}$ & $\begin{array}{l}\text { encephalitis. } \\
\text { otitis. } \\
\text { adenitis. } \\
\text { endocarditis. } \\
\text { colitis. } \\
\text { enteritis. } \\
\text { nephritis. }\end{array}$ \\
\hline
\end{tabular}




$\begin{array}{lll}\text { NAME } & \text { ROOT WORD } & \text { INFLAMMATION OF } \\ \text { liver } & \text { hepar } & \text { hepatitis. } \\ \text { mouth } & \text { stoma } & \text { stomatitis. } \\ \text { muscle } & \text { mys, myos } & \text { myositis. } \\ \text { nerve } & \text { neuron } & \text { neuritis. } \\ \text { nose } & \text { rhis, rhinos } & \text { rhinitis. } \\ \text { rectum } & \text { procto } & \text { proctitis. } \\ \text { skin } & \text { derma } & \text { dermatitis. } \\ \text { stomach } & \text { gaster } & \text { gastritis. } \\ \text { tongue } & \text { glossa } & \text { glossitis. }\end{array}$

\section{GLOSSARY}

Abdomen.-The portion of the trunk extending from the chest to the pelvis.

Abnormal.- Not conforming to the general rule of nature.

Abduct.-To draw from median line.

Adduct.-To draw toward center.

Abrasion.- The rubbing off of the skin or mucous surfaces by injury.

Adenitis.-Inflammation of a gland.

Adenoid.-A polypoid growth in the pharynx, back of the nose.

Adipose.-Consisting of fat.

Adolescence.-The period between puberty and full development.

Aerated.-Exposed to the action of fresh air.

Albumen.-White of egg.

Alkaline.-Having properties the opposite to those of an acid.

Alopecia.-Baldness.

Alveoli.-Air cells of the lungs.

Analyze.-To ascertain the composition of.

Anasarca.-General dropsy.

Anatomy. - The study of the different tissues and organs of the body.

Anemia.-A decrease in the blood constituents.

Anesthetic.-Pertaining to the loss of sensation.

Ankylosis. - A locking of a joint from injury or disease.

Anomalies.-Marked deviation from the normal.

Anorexia.-Loss of appetite.

Antiseptic.-Having the power to destroy bacteria and to prevent their growth.

Anus.-The external opening of the rectum.

Areola.-A colored ring around an object.

Arthrepsia.-Marasmus.

Asepsis.-Absence of bacteria.

Asphyxia.-Suffocation.

Assimilate.-The process of transforming food into such a nutrient condition that it may be taken up by the blood. 
Atony.-Want of power, especially muscular power.

Atresia.- The absence of the natural opening to a normal canal.

Auricle.-The external ear.

Autopsy.-An examination of the organs and tissues of the body made after death.

Bacteria.-Germs. A low form of plant life. They multiply very rapidly.

Batting.-Cotton or wool arranged in layers for quilting.

Bicuspids. - The fourth and fifth teeth from the middle.

Bladder.-The reservoir for the urine.

Bronchial Tubes. - The air-passages from the windpipe or trachea to the air-cells of the lungs.

Cachexia.-A very low condition of nutrition due to some serious disease.

Calorie.-The amount of heat required to raise I gram of water tc $I^{\circ}$ of heat Centigrade.

Canine Teeth. - The eye teeth, third from the middle.

Canthus.-The angle formed by the upper and lower eyelids at the internal and external extremity of the palpebral fissure.

Carbohydrates. - Sugars.

Caries. - Death of a bone.

Caseate.-To break down and form a cheese-like mass, seen in tubercular processes.

Casein.-The ingredient of milk which constitutes most of the curd, and is the chief source of proteid.

Catharsis. - To purge.

Catheter.-A hollow, flexible rubber tube used to draw off the urine from the bladder (catheterization).

Cell. - The smallest division of animal life. The entire body is composed of millions of cells.

Cereals. - The grain plants, such as wheat, rye, barley, etc. The seed is used for food.

Cerumen.-The wax of the ear.

Cicatrix.-Scar tissue.

Circulation.-The flowing of the blood through the body.

Clonic.-The term given to intermittent convulsions.

Coagulate.-To thicken, clot, or curdle.

Coalescence.-The union of two or more parts of a thing. To flow together.

Colic.-Severe griping pain in the abdomen. 
Colostrum.-The milky fluid which can be pressed from the breasts of a pregnant $w$ man, and which flows for the first three days after the birth of the child.

Communicable.-Contagious.

Complication.-A condition occurring during the course of a disease.

Compound.-Composed of two or more ingredients.

Compress.-A folded cloth, wet or dry, applied to a part for the relief of inflammation, or to prevent a hemorrhage.

Condiment.-Substances used to give relish to food.

Congenital.-Being present at the time of birth.

Congestion.-An abnormal accumulation of blood in an organ or part of the body.

Constipation.-Difficult or infrequent bowel movements.

Constriction.-The state of being squeezed.

Contagious. - Capable of direct communication.

Contaminated.-Rendered impure by contact.

Contusion.-A bruise.

Convalescence.-The gradual return to health after sickness.

Convulsion.-A violent and involuntary muscular contraction, or series of contractions.

Cornea.-The transparent anterior portion of the eyeball.

Coryza.-Cold in the head.

Coxalgia.-Tubercular hip-joint disease.

Crepitus.-A grating, crackling sound.

Curdle. - The formation of curds.

Curds. - The thickened portion of milk.

Curetment.-Scraping of a part to remove diseased tissue.

Cyst.-A cavity containing fluid and surrounded by a capsule.

Cystitis.-Inflammation of the bladder.

Dandruff.- Small scales from the scalp.

Deaf-mutism. - The condition of being both deaf and dumb.

Debilitated.-Weakened.

Decoction. - The water in which a substance has been boiled.

Decubitus. - The position of a patient in bed.

Defecate. - The act of having a bowel movement.

Deformity.-Unnatural shape.

Degeneracy.- The tendency to deteriorate.

Deglutition.-The act of swallowing.

Deleterious.-Injurious.

Delivery.-The birth of a child.

Dentition.-The process of cutting teeth. 
Dermatitis.-Inflammation of the skin.

Desquamate.-To shed the skin.

Development.-A gradual growth through progressive changes.

Diagnosis.-Recognition of a disease.

Diastole.-The period when the chambers of the heart dilate after the period of contraction. Occurs after each heart-beat.

Digestion.-The process of changing the food from the form in which it enters the body to that in which it is absorbed by the blood.

Disinfection.-Rendering free from germs.

Diurnal.-Daily.

Douche.-A jet of water entering a cavity of the body.

Dyspepsia.-Chronic indigestion.

Ecchymosis.-Extravasation of blood into surrounding tissues.

Edema.-Dropsical swelling.

Effervescent.-Bubbling up, with the giving off of gas bubbles.

Effusion.-The pouring out of a serous or bloody fluid into the tissues or cavities of the body.

Embolus.-A particle of fibrin or other material brought by the blood current and forming an obstruction within an artery at its place of lodgment.

Embryo.-The unborn child before the fourth month of pregnancy.

Emulsion.-A mixture of an oily substance with a liquid.

Enema.-An injection into the rectum.

Epidermis.-The outer layer of the skin.

Epistaxis.-Nose-bleed.

Eruption.-A rash.

Eustachian Tube.-A duct running from the middle ear to the pharynx.

Evaporation.-Converting into vapor.

Excretion.-A discharge of waste products.

Exhale.-To breath out.

Expiration.-The emptying of the lung of air.

Fat-free Milk.-Milk from which all the fat has been removed.

Feces.-The stools. Matter expelled from the intestines by way of the rectum.

Fetus.-The unborn child after the fourth month of pregnancy.

Fissures.-Cracks in the skin, or mucous membrane.

Flatulence.-The presence of gas in the stomach and intestines.

Flocculent.-Flaky.

Fomentation.-Flannel cloths rung out of hot water and placed on the body as a means of applying moist heat. 
Fontanel. - The soft spot in a child's head, caused by the non-union of the bones.

Formula.-A list of the names and quantities of the ingredients of a mixture.

Function.-The mode of action of an organ.

Furuncle.-A boil.

Genital.-Pertaining to the organs of reproduction.

Gland.-An organ of the body that secretes substances of use to the system or casts off waste matter.

Hemorrhage.-Bleeding.

Hemorrhoids.-Piles.

Hepatization.-Liver-like. Used in describing the lung in pneumonia.

Hernia.-A rupture. The protrusion of the internal organs from their natural position.

Hydrotherapy.- Treatment by means of water.

Hygiene.-The science of preserving health.

Hypertrophy.-To enlarge by overgrowth.

Icterus.-Jaundice.

Idiocy.-A lack of mental understanding.

Imbecile.-One who is mentally weak.

Incisors.-The four front teeth of each jaw.

Incubator.-An apparatus for preserving the life of a premature infant.

Infected.-Brought in contact with bacteria.

Inherent.-Instinct. The ability to perform certain acts without knowledge of the reason and without previous training of the individual.

Insomnia.--Sleeplessness.

Inspiration.- The act of filling the lungs with air.

Intermittent.-Ceasing at intervals.

Interstitial Tissue.-The supporting tissue or framework of an organ or structure of the body.

Isolation.-The complete separation from other individuals.

Kumiss.-Fermented milk.

Labor.-Childbirth.

Laceration.-A cut.

Lancinating.- Shooting. 
Latent.-Hidden.

Laxative.-A medicine that moves the bowels gently.

Leukocytosis.-An increase in the number of white blood corpuscles in the circulation.

Ligament.-A band of tissue binding two parts together.

Lime-water.-A solution of lime in water.

Loins.-The lower part of the back and region of the hips.

Lubricant.-An oily material used to make two surfaces glide smoothly over one another.

Malaise.-A feeling of weakness. Listlessness.

Manipulation.-The act of handling or working with the hands.

Massage.-A rubbing or kneading of the muscles.

Mastication.-The act of chewing.

Membrane.-A thin lining tissue.

Membranous Croup.-Diphtheria of the larynx.

Meningitis.-An inflammation of the membranes covering the brain and spinal cord.

Microbe.-A germ.

Micturition.-The act of urinating.

Milk Sugar.-A sugar made by the evaporation of the whey of milk.

Milk Teeth.-The first set of teeth.

Minim.-About a drop. One-sixtieth of a fluid dram.

Molars. - The back teeth.

Morbid.-Diseased.

Morbid Anatomy.-The study of diseased tissues.

Mortality.-The frequency of death.

Mucous Membrane.-The lining membrane of all passages and cavities that come in contact with the air.

Mucus.-A slimy fluid from the mucous membrane.

Nave1.-The umbilicus.

Necrosis.-Death of a structure or tissue.

Nephritis.-Inflammation of the kidney. Bright's disease.

Neural.-Pertaining to a nerve.

Neuralgia.-Pain along the course of a nerve.

Neurosis.-A nervous functional disease.

Neurotic.-Nervous.

Nevus.-A birth-mark.

Nitrogenous Food-stuffs.-Meats, potatoes, and similar foods.

Nocturnal.-Pertaining to night.

Normal.-According to the rule of nature. 
Obstetrics.-The management of childbirth.

Occluded.-Closed.

Opaque.-Not transparent.

Organism.-The body as a whole.

Organize.-The conversion into living tissue.

Palatable.-Agreeable to the taste.

Parasites. - Insects living on animals, such as lice.

Paroxysm.-A spasm.

Paroxysmal.-Spasmodic.

Parturition.-Childbirth.

Pasteurization.-The heating of milk to $167^{\circ} \mathrm{F}$. to destroy germs.

Pathology.-The science of the changes which take place in the structure of the body in disease.

Patulous.-Open.

Pelvis.-The bony basin supporting the abdominal viscera.

Percentage.-Rate per hundred.

Perforation.- Used to denote the occurrence of a hole into an organ or through the bowel.

Period of Incubation.- - The time elapsing between the introduction of bacteria into the body and the appearance of the symptoms of the disease.

Periodic.-Recurring at intervals.

Peristalsis. - The worm-like movements of the intestines by which the feces are moved.

Peritonitis. - An inflammation of the membrane lining the abdominal cavity.

Pertussis.-Whooping-cough.

Petechiæ. - Hemorrhagic spots in the skin.

Phenomenon.-A thing that is observed.

Physiology.-The science of the functions of the different organs.

Placenta. - The attachment of the umbilical cord to the inner side of the womb. The "after-birth."

Pneumonia.-Inflammation of the lungs.

Polyp.-A tumor composed of mucus.

Pores.-The openings of the sweat-glands in the skin.

Poultice.-A hot, soft mass, used to apply moist heat or to remove odor.

Predisposition.-A tendency to.

Pregnancy.-The carrying of the child by mother before birth.

Premature.-Before full term. 
Prophylaxis.-Measures to prevent the development or spread of disease.

Proteid.-The albuminous foods; the nourishing part of milk, eggs, and meat.

Pruritus.-Itching.

Puberty. - The period of life at which an individual becomes capable of producing children.

Pubic.-Pertaining to the front of the pelvis.

Puerperium.-The period immediately following childbirth.

Pulse-rate.-The number of beats per minute.

Purgative.-A medicine that cleans out the bowels.

Quarantine.-The guarding of a building which houses a contagious case, so that no one can enter or leave.

Rash.-A breaking out on the skin.

Ratio.-Proportion.

Reaction.-The return to normal after collapse. The return to warmth after a chill.

Rectum.-The lower end of the intestines.

Regurgitation.--Vomiting of mouthfuls.

Relax.-To make less rigid.

Remittent.-Temporary disappearance.

Respiration.-The act of breathing.

Respiratory Rate.-The number of respirations per minute.

Resuscitate.-To revive.

Rickets. - A disease of childhood characterized by deformity of the bones and changes in the liver and spleen.

Rigor.-Chill.

Rigor Mortis.-The stiffening of the muscles after death.

Rötheln.-German measles.

Rubella.-Measles.

Rubeola.-Measles.

Saliva.-Secretion present in the mouth.

Saturated Solution.-A solution of a substance in which no more of that substance can be dissolved.

Sclerosis.-Hardening of a part due to overgrowth of fibrous tissue.

Scrofulous.-Tubercular.

Scurvy.-A disease due to a lack of nourishing diet.

Secretion.-The substance produced by glandular action. 
Sedentery. - Sitting.

Sepsis.-Poisoning by germs.

Sequel.-A condition which appears after a disease.

Shock.-The period of collapse following an accident or operation.

Sinus.-Discharging channel from an abscess cavity.

Sitz Bath.-Sitting in water covering the hips.

Sordes.-Crusts that accumulate on the teeth.

Spasmodic.-Occurring in spasms.

Specific Remedy.-One that has a distinct curative influence on an individual disease, as quinin in malaria.

Sputum.--Spittle.

Sprain.-A tearing of the ligaments around a joint.

Stenosis.-Constriction or narrowing of a channel.

Sterilize.-To render free from germs.

Sterile.-Absence of germs.

Stimulate.-To excite action.

Stomach Teeth.-The two milk teeth on either side of the four lower incisors.

Striæ.-Lines or furrows.

Structure.-Construction of parts.

Stupe.-A cloth rung out of hot water and used for applying moist heat.

Suppression.-A stoppage of a discharge.

Symptom.-A sign of a disease.

Temperature.-The degree of heat.

Tetanus.-Lock-jaw.

Tissue.-A collection of cells of the body doing the same work.

Toxin.-A poison.

Traumatism.-An injury.

Tumor.-An abnormal swelling.

Umbilical Cord.-The cord by which the infant is attached to the placenta. It enters the child's body at the umbilicus or navel.

Unhygienic.-Contrary to the laws of health.

Urine.- The excretion of the kidneys.

Uterus. - The womb.

Vapor Bath. -A bath in vapor used to produce sweating.

Vagina.-The opening in the female which extends from the womb to the outer parts. 
Varicose Veins.-Swollen, thickened veins.

Venous Stasis.-Engorgement of the veins with blood.

Ventilation.-The process of replacing foul air with pure.

Viscera.-The organs of the body.

Vitality.-Vigor.

Vulva.-The external genitals of the female.

Weaning.-Removing the nursing infant permanently from the breast.

Whey.-The part of milk which remains fluid after the curds have formed. 



\section{INDEX}

ABbReVIATIONS, 444

Abscess, cerebral, 197

in Pott's disease, 279

ischiorectal, $15 \mathrm{I}$

of liver, 153

of lung, 97

psoas, 279.

retropharyngeal, I 15

Accommodation, 228

Adenitis, 24I

tubercular, 22, 282

Adenoid, I ro

Air-beds, 430

Air-cushions, 430

Airing of newborn babe, 32

Albumin and milk, 338 in urine, test for, 213 water, 338

Albuminuria, 212

Alkaline baths, 407

Amyloid kidney, 220

Anemia, 21, 177

Anesthesia, 187

Aneurysm, 21, I 75

Angina, follicular, Io9

Vincent's, I 12

Anginoid scarlet fever, 286

Animal parasites, 148

Ankle clonus, I86

Ankylosis, 280

Anorexia, 103

Antidotes of poisons, 387

Antiseptic poultices, 399

Antitoxin in diphtheria, 298

Anuria, 2 I I

Anus, atresia of, 129 fissure of, ${ }_{5}$ I

Aortic insufficiency, 168 stenosis, I68
Apex-beat, I59

Aphasia, 198

Aphthous stomatitis, I05

Apoplexy, I97

Apothecaries' weight, 439

and metric system, relation botween, 440

Appendicitis, 142

Appetite in digestive diseases, IO2

Apple gruel, 340

Arachnoid, 184

Arrow-root gruel, 342

with egg, 343

Arteriosclerosis, 2 I, I 75

Arthritis, tubercular, $28 \mathrm{I}$

Arthropathies, I87

Artificial feeding, 348

respiration, $42 \mathrm{I}$

Ascaris lumbricoides, I49

Ascites, I54

Asphyxia, emergency treatment, $3^{89}$ in newborn, 423

Aspiration in empyema, 77 of chest, 437 pneumonia, 97

Asthma, 68 emergency treatment, 389

Astigmatism, 228

Atelectasis, $7 \mathrm{I}$

Athetosis, I86

Atmosphere, moist, maintaining of, 44

Atomizers, 42

Atresia of anus, 129

Atrophic rhinitis, 55

Atrophy, infantile, 326 muscular, 206

Auscultation of heart, 160

Autumnal fever, 259 
BABIEs, blue, 20, 159

Babinski's reflex, i 87

Bacterines, 420

Balanitis, 225

Baner's formula for cream mixtures, 356

Barley gruel, 34r

jelly, 342

maltine, and milk mixture, 343

water, 337

Barlow's disease, 323

Bath, alkaline, 407

bran, 407

compound glycerin, 408 sulphur, 408

for skin diseases, 407

graduated cold, 396

hot, 402

hot-air, 405 sitting, 406

hygienic, 409

linseed, 408

mustard, 409

prevention of chills after, 43

salt, 406

shower, 409

sitz, 406

sponge, 395,409

starch, 408

tar, 409

tepid, 409

vapor, 403

vinegar and mercury, 409

Bathing in childhood, 32

in infancy, 32

of newborn, $3 \mathrm{I}$

Bed-sores, 268

Beef juice, 343 and milk, 343

Bell's palsy, 207

Bichiorid baths, 435

Birth-marks, 238

Birth palsy, 2r, 106

Bites, treatment of, 389

Bladder, diseases of, 223

exstrophy of, 224

Blebs, 236

Bleeders' disease, 321

Blindness, congenital, $2 \mathrm{I}$

Blister, cantharides, 397

Blood, diseases of, 175 specific gravity of, 176
Blood-vessels, diseases of, 175

Blue babies, 20, I 59

Bones, tuberculosis of, 277

Borborygmi, I 34

Bottles, feeding, 366

sterilization of, 42

Bottom milk, 353

Bowing of tibia, 24

Bow-legs, 24

Bradford frame, Fauntleroy's modification of, $43 \mathrm{I}$

for fractures, 431

Bradycardia, 161

Brain, 179

abscess of, 197

diseases of, 193

hemorrhage of, 21

malformations of, 188

Bran baths, 407

Breasts of newborn, 32

Breath in digestive diseases, 102

Breathing, stridulous, $5^{8}$

Bright's disease, 216

Bronchiectasis, 67

Bronchitis, 19, 62

acute, 62

capillary, 19

chronic, 66

Bronchopneumonia, 19, 20, 84 acute, 87 tuberculous, 274

Bubble quick, 368

Buck's extension, application of, 425

Bulimia, I03

Burns, dressing of, 436 emergency treatment, 389

Buttermilk, 345 care of, in house, 365

conserve, 345

Calculus, renal, 220

vesical, 224

Camphorated oil, 398

Cantharides blister, 397

Capillary bronchitis, In

Carbohydrates, 330

Care of milk in house, $3^{64}$

Catalepsy, 187

Catarrhal laryngitis, $5^{6}$ stomatitis, 105

Catheterization, 437 
Cautery, Paquelin, $43^{8}$

Cephalodynia, 3 I9

Cerebral localization, I94 meningitis, 189 paralysis, I96 pneumonia, 96

Cerebrospinal fever, 245 meningitis, 189,245

Chapin dipper, 355

Characteristic cry, 26

Charcoal poultice, 399

Chest, aspiration of, 437

Cheyne-Stokes respiration, $5^{\mathrm{I}}$

Chicken-pox, 307

Child, crowing, 6o

Childhood, general hygiene of, 33 nursing in, 29 period of, $\mathrm{r}$ I

Chills, emergency treatment, $3^{89}$ prevention of, after baths, 43

Chlorosis, 178

Cholecystitis, ${ }^{52}$

Cholera infantum, I40 morbus, 142

Choluria, 2 I 2

Chorea, 21, I99 heart murmur in, 20

Choreiform movement, 186

Chyluria, 2 I 2

Circulation, $\mathrm{I}_{57}$ fetal, $5^{8} 8$

Circulatory system, anatomy of, I 55 diseases of, 20

Cirrhosis of liver, ${ }^{5} 53$

Cleft palate, ro4

Clothing in childhood, 33

in infancy, 33

of newborn, $3 \mathrm{r}$

Clubbed hands, 24

Club-feet, 24

Coffee-ground vomit, I 2 I

Cold bath, graduated, 396 compresses to rectum, 42 in the head, $5^{2}$ pack, 396

Colic, intestinal, 129 renal, 220

Collapse, treatment of, $3^{89}$

Colon, irrigation of, 396, 4I 7

Colostrum, 332

Coma, 187

Compensation, cardiac, 169
Compensation, period of, 169

Compresses, $40 \mathrm{I}$ cold, to rectum, 42

Condensed milk, 346 care of, in house, $3^{6} 5$

Congestion of liver, $15^{2}$ of lungs, 83

Conjunctivitis, 21, 233

Constipation, r 27

Constitutional diseases, 22

Contagious diseases, 22, 284 acute, heart murmur in, 20 definition of, 245 nurse in, 44

Continuous saline injection, 418

Contra - indications for various drugs, 379

Convulsions, 2 I, 185,195 emergency treatment, 390

Cord, care of, $3 \mathrm{I}$

Corrosion of esophagus, II 8

Coryza, $5^{2}$

Cough, 46

Counter-irritation, 397

Cowpox, 308

Cow's milk, 334

Coxalgia, 279 extensions for, 425

Coxitis, 279

Cradles, improvised, 44

Cranial nerves, 184

Cream, 334

dipping for, 354

in artificial feeding, $35^{2}$

method for changing percentage of, 355

siphoning for, 353

Cretinism, 197

Crossed paralysis, I95

Croup, emergency treatment, $3^{89}$

kettle, 4I4

spasmodic, 57

tent, $4^{\mathrm{I}} 3$

Croupous exudate, 93 pneumonia, 20, 84, 9I

Crowling's rule for dosage, 378

Crusts, 236

Cry, characteristic, 26

Cuffs, 433

Cups, dry, 398

wet, 399

Cyclic vomiting, I2I 
DACTYLITIS, tubercular, 282

Deaf-mutism, 198

Death, cause of, 27

Defervescence, 242

Deformities, 23

Diabetes insipidus, 214 mellitus, 23, 320

Diaphragmatic pleurisy, 79

Diarrhea, 129, I30

in typhoid fever, 265

summer, 132

Diastole, 167

Dicrotic pulse, I6I

Digestion, 25

Digestive tract, diseases of, IOI

Digitalis poultice, 399

Dilatation of heart, 173 of stomach, 125

Diphtheria, 294

Dipper, Chapin, 355

Dipping for cream, 354

Diseases, inheritance in, I 8 peculiar to children, 17

Disinfection, 435

in scarlet fever, 287

of excreta, 436

of hands, 435

of stools in typhoid fever, 269

Dislocation, congenital, of hip, 24 emergency treatment, 390

Dosage, rules for, 378 rule for reduction of, $44 \mathrm{I}$

Douching, vaginal, 420

Draughts, avoidance of, $4 \mathrm{I}$

Dropsy in heart disease, 162

Drowning, emergency treatment, 390

Drugs, characteristic pulses of, $38 \mathrm{r}$ dominant action of, 380 poorly borne by children, 377 used in children's diseases, with dose, $3^{8} 3$

well borne by children, 377

which cause eruptions, $38 \mathrm{I}$

color stools, 382

color urine, 382

contract pupil, $3^{81}$

dilate pupil, $3^{81}$

quicken pulse, $3^{80}$

raise blood pressure, 381

slow pulse, $3^{81}$

Dry cups, 398
Ductus arteriosus, 159

Dura mater, 183

Dysentery, 138

Dyspepsia, atonic, 123

catarrhal, I 23

chronic, 122

nervous, 122

Dysphagia, I03

Dyspnea, 48

emergency treatment, 39 I

EAR, diseases of, 21,228 nursing in, 234

Examination of, 43

foreign bodies in, $39 \mathrm{I}$

running, 21

syringing, 410

Earache, 233 emergency treatment, 39 I

Eczema, 22, 236

Edema of glottis, 6 r pulmonary, $7 \mathrm{I}$

Electricity, 435

Embolism, I 7 I

Emergencies, treatment of, $3^{89}$

Emphysema, pulmonary, $7 \mathrm{r}$

Empyema, 75

Encephalocele, I 88

Endocarditis, I64 after rheumatism, 2 I

complicating rheumatism, 23

fetal, 159

ulcerative, I 70

Endocardium, 156

Enemata, 4ro

Enterocolitis, 138

membranous, 139

Enuresis, 21, 223

Epilepsy, 198 Jacksonian, 194

Epileptiform convulsions, 185

Epiphysitis, 282

Epispadias, 224

Epistaxis, 55

treatment, 391

Erb's paralysis, 196

Erysipelas, 310

Erythema, 236

Esophagus, corrosion of, II 8 diseases of, 118

stricture of, 118 
Eustace Smith's sign, 277

Examination, method of holding child for, 4r

Examinations, general, 36

Excreta, disinfection of, 436

Exercise, 375 in childhood, 34

in infancy, 33

Expectoration, 48

Exstrophy of bladder, 224

Extension, Buck's, 426

Extensions for fractures, 425

Extremities, growth of, 14

Eye, care of, 229 compresses for, $40 \mathrm{I}$

diseases of, 21, 227

foreign bodies in, 230

malformations of, 229

syringing, 409

Eyelid, everting, 230

FAINTING, treatment, 39I

Farina gruel, 34I

Fastigium, 242

Fat percentages in mixtures, rules for, 363

Fats, 329

in artificial feeding, $35^{\circ}$

Fatty degeneration of heart, I 73

Fauntleroy's modification of Bradford's frame, $43^{I}$

Febricula, 243

Feces, 127 incontinence of, 151 milk, 127

Feeding, adjuncts to, 375 artificial, 348

by stomach-tube, 416 during first year, 369 schedule for, 368 second year, $37 \mathrm{I}$

infant, $3^{28}$ regurgitation after, 43 rules for, 367

Feet, care of, 43

Fehling's solution, 2 I 4

Female genitals, diseases of, 225

Fetal circulation, 158

Fever, cerebrospinal, 245

characteristics of, 242

continued, 242
Fever, hay-, 69

hectic, 242

inanition, 26

infectious, 242

intermittent, 242

malarial, 248

remittent, 242

scarlet, 284

types of, 242

typhoid, $25^{8}$

Fibrinous bronchitis, 66

Fibroid heart, 172

Fingers, six, 24

webbed, 24

Fissure of anus, $15 \mathrm{I}$

Flaxseed poultice, 400

Flexner's serum, 247

Flint murmur, I6r

Floating kidney, 22 I

Flour gruel, 34I

Fontanels, anterior, closing of I4 posterior, closing of, 14

Food, constituents of, 328

Foot-baths, hot, $40 \mathrm{I}$

mustard, 407

Foramen ovale, ${ }_{5} 8$ patulous, 159

Fracture of femur, dressing for, $4_{2} 6$

Fracture-box, 429

Fractures, emergency treatment, $39^{2}$

extensions for, 425

Frames, 43I

Frenum, ulcer of, 104

Friction sound, r6o

Friedreich's ataxia, 206

Fumigation, 435

Furuncle of auditory canal, 233

Furunculosis, 237

GAIT, scissors, 203

Gall-stones, 152

Gangrene of lung, 97

Gastralgia, I 24

Gastric ulcer, I 24

Gastritis, 122 chronic, I 22

Gastro-intestinal disorders, 20

Gavage, 416

Gelatin, preparation of, for infantile diarrhea, 343 
Genital tract, malformation of, 224

Genitals, diseases of, 224 of newborn, care of, $3^{2}$

Genito-urinary system, malformations of, 2 I

diseases of, 21

Genu valgum, 24 varum, 24

German gruel, 34 I

Gingivitis, 104

Girdle pain, 203

Glandular system, diseases of, 21, 239

Glomeruli of kidneys, 209

Glossary, 446

Glossitis, 104

Glottis, edema of, 6 I

Glycerin bath, compound, 408

Glycosuria, 2 I 2

Gonorrhea, 225

Grand mal, I98

Gray tubercle, 27.5

Griffith's weight chart, I 2

Growing pains, 23

Growth during first year, 14 of extremities, 14

Gruel, apple, 340

arrow-root, 342

barley, 34I

farina, $34 \mathrm{I}$

flour, 341

German, 34I

oatmeal, 340

rice flour, $34^{2}$

Gums, inflammation of, 104

HАBIT, cry of, 26 spasm, 201

vomiting, I 2 I

Hamilton squint, 426

Hands, clubbed, 24

disinfection of, 435

Hare-lip, 104

Harrison's groove, 324

Hay-fever, 69

Head, circumference of, I4

Hearing, development of, 14

Heart, action of, 159

anatomy of, 155

congenital malformations of, 20 dilatation of, 173
Heart, diseases of, 20 nursing in, 173

failure, emergency treatment, 392

fatty degeneration of, 173

fibroid, 172

hypertrophy of, 172

malformations of, I 59

murmur in chorea, rheumatism, and acute contagious diseases, 20

sounds of, 160

transposition of, 159

Height, I I

at birth, II, 14

Heller's test, 213

Hematemesis, I 2 I

treatment, 393

Hematozoa, 249

Hematuria, 212

Hemiplegia, 185

Hemocytometer, i 76

Hemoglobin, 176

Hemoglobinometer, ${ }_{17} 6$

Hemoglobinuria, 212

Hemopericardium, 164

Hemophilia, 321

Hemoptysis, 7o treatment, 393

Hemorrhage from bowel, treatment, 393

from mouth, treatment, 393

from nose, treatment, 391

of brain, 21

Hemorrhoids, $15 \mathrm{I}$

Hepatization, gray, 93 red, 92

Hereditary syphilis, 22, 253 cry of, 26

Heubner's mustard bath, 397

Hiccough, 12 I

Hip, congenital dislocation of, 24

Hives, 238

Hodgkin's disease, 241

Hook-worm, i 50

Hordeolum, 228

Horseshoe kidney, 2 10

Hot application, 401

bath, 402

foot-baths, $40 \mathrm{I}$

pack, 402

modified, 403

Hot-air bath, 405 
Hot-air bath, sitting, 406

Hot-water bags, $40 \mathrm{I}$ care of, 42

Human milk, 33 I

Hunger, cry of, 26

Hutchinson's teeth, I6, 254

Hydrocele, 225

Hydrocephalic cry, I9I

Hydrocephalus, I89, 197 cry of, 26

Hydro-encephalocele, I88

Hydronephrosis, 22 I

Hydropericardium, I64

Hydrophobia, 256

Hydrothorax, 80

Hygienic management of children in general, 30

Hyperemia, renal, 2 I 4

Hyperesthesia, I87

Hypermetropia, 228

Hyperpyrexia, 242

Hypertrophic rhinitis, 55

Hypertrophy of heart, I 72

Hypodermics, 4I9

Hypodermoclysis, 424

Hypospadias, 224

Hypostatic pneumonia, 97

Hysteria, 199

Hysteroidal convulsions, 185

ICE-CAP, 395

Icterus neonatorum, $\mathbf{I}_{52}$

Ileocolitis, 138

Impacted cerumen, 233

Impetigo contagiosa, 238

Inanition fever, 25

Incontinence of feces, ${ }_{5} 5$

Incubation, period of, 243

Incubators, 34

Indicanuria, 2 I 2

Indigestion, acute gastric, I 2 I cry of, 26

intestinal, I 30

Infancy, general hygiene of, 32 period of, I I

Infant feeding, 328

foods, proprietary, 347

receipts for, 337

Infantile atrophy, 326

paralysis, 203

Infectious disease, definition, 245
Infectious disease, nurse in, 44

fevers, 242

acute, 22

nursing in, 256

Inflammatory rheumatism, 3 I 7

Influenza, 3 i 5

Inhalations, technic of, 4 I 3

Inheritance in diseases, 18

Injections, intravenous, 424 vaginal, 420

Insufficiency, aortic, I68 mitral, I 68 pulmonary, 169

tricuspid, I 68

Intermittent fever, quartan, 249 quotidian 249

malarial fever, $25^{\circ}$

Intestinal colic, I 29 indigestion, I 30 obstruction, I 45 perforation in typhoid fever, 260 , 265

Intestines, diseases of, I 27

malformations of, I 29

obstruction of, I 45

tuberculosis of, 142

Intravenous injections, 424

Intubation, 302

Intussusception, I 45

Invasion stage of fever, 242

Iritis, 228

Irrigation of colon, $4 \mathrm{I} 7$ cold, $39^{6}$

rectal, 42

Ischiorectal abscess, ${ }_{5} \mathrm{I}$

Itch, 238

JACKSONIAN epilepsy, I94

Jaundice, I 52 catarrhal, ${ }^{5}{ }^{2}$

Joints, tuberculosis of, 277

Junket, 346

KERATITIS, 228, 230

Kernig's sign, I92

Kidney, amyloid, 220 anatomy of, 209 after scarlet fever, 2 I diseases of, nursing in, 222 floating, 22 I 
Kidney, horseshoe, 2 10 malformations of, 2 Io sarcoma of, 222 tuberculosis of, 221

Knee-jerks, I 86

Knock-knee, 24

Koplik's sign, 292

Kumiss and bean flour, 346

Kyphosis, 279, 324

LACUNAR angina, 109

La grippe, 3 I 5

Laminated teeth, I6

Landry's paralysis, 206

Laryngismus stridulus, 2 I , 60 emergency treatment, 393

Laryngitis, 56 acute catarrhal 56 syphilitic, 57 tubercular, 57

Lavage of stomach, 414

Leukemia, 178

Leukocytosis, I 78

Lice, 238

Linseed bath, 408

Lisping, 20I

Lithuria, 2 I I

Liver, cirrhosis of, 153 congestion of, 152 diseases of, 152

Lobar pneumonia, 84, 9 I

Lockjaw, 255

Lumbago, 319

Lumbar puncture, 192

Lung, abscess of, 97 anatomy of, 8I diseases of, 8 I congestion of, 83 gangrene of, 97

Lupus vulgaris, 238

Lymph-glands; bronchial, tuberculosis of, 277

Lymphatism, 240

Lysis. 242

Macules, 235

Malaria, 248

Malarial fever, 22, 248

Male genitals, diseases of, 225

Malt soup mixture, 344

Marasmus, 23, 326

cry of, 26

Masks for skin diseases, 434

Massage, 434

Mastoid disease, 2 I

Mastoiditis, 233

Measles, 290

Meconium, 127

Medical terminology, 445

Meninges of brain, 183

diseases of, 189

Meningitis, 189

cerebral, I 89

cerebrospinal, 189,245

cry of, 26

tubercular, 2 I, 190

Meningocele, I 88

Mercurial stomatitis, Io7

Metric system, 440

Microcephalus, 188

Miliaria, 236

Miliary tuberculosis, 276

Milk, bottom, 353

care of, in house, 364

condensed, 346 care of, in house, 365

cow's, 334

digestion of, I I9

examination of, 333

feces, 127

infection, 133

methods of modifying, 356

pasteurized, 335

peptonized, 339

rice, 337

siphoning, 353

skimmed, 353

sterilized, 336

teeth, average age of eruption of 14

woman's, 331

Mineral salts in food, $33 \mathrm{I}$

Minim, method of obtaining fractional part, 442

Mitral insufficiency, 168

stenosis, 167

Mixtures, top-milk, $35^{8}$

Modifying milk, methods of, 356

Moist atmosphere, maintaining, 44

Monoplegia, 185

Mouth, diseases of, 104 inflammation of, 105 
Mouth, malformation of, I03 of newborn, care of, $3^{\mathrm{I}}$ syringing of, $4 \mathrm{I} 3$

Mumps, 3I4

Murmur, cardiac, I60 heart, in chorea, rheumatism, and acute contagious diseases, 20

Murmurs in endocarditis, 166

Muscular rheumatism, 318

Mustard bath, 407 Heubner's, 397

foot-bath, 407

pack, 398

paste, 397

plaster, 397

poultice, 397

Myelitis, 202

Myocarditis, 172

Myopia, 228

NASAL syringing, 4II

Nebulizers, 42

Nephritis, 2 I6 chronic interstitial, 218

parenchymatous, 217

Nerves, cranial, I 84

diseases of, 206

- spinal, I84 sympathetic, 184

Nervous system, anatomy of, I79 diseases of, $2 \mathrm{I}$ nursing in, 207 diseases of, peculiar in children, 187

Nervousness, treatment of, 393

Neuritis, 206 multiple, 206

Nevus, 238

Newborn, asphyxia of, 423 bathing of, $3^{\mathrm{I}}$ care of, 30 clothing of, $3 \mathrm{I}$ period of, II

Night terrors, 202

Nipples, 366 babies, care of, 43

Nodding spasm, 2I, $20 \mathrm{I}$

Noma, I06

Normal salt solution, 387

Nose, foreign bodies in, $39^{2}$
Nosebleed, treatment, 39I

Nursery, general hygiene of, 34

Nursing in childhood, 29

Nutrient enemata, 419

Nutrition, 328

Nutritional diseases, 22, 323

Nystagmus, 2I, 20I, 23I

OATMEAL gruel, 340 jelly, 343 water, 337

Obstetric hand, 200

Obstruction, intestinal, 145 chronic, 147

Oiled silk jacket, 43I

Onychia, 238

Orthopnea, 49

Operation, preparation for, 436

Ophthalmia, 2 I neonatorum, 229

Opisthotonos, I9I

Orthopedics, 23

Osteomyelitis, tubercular, 28I

Otitis media, 2I, 233

Oxyuris vermicularis, I49

PACK, hot, 402

Pain, cry of, 26

Palpitation of heart, 162

Palsy, Bell's, 207

Pap, 34I

Papules, 235

Paquelin cautery, 438

Paralysis, 2 I

birth, 2I

cerebral, 196 $_{9}$

crossed, I95

definition of, 185

Erb's, 196

infantile, 203

Landry's, 206

post-diphtheritic, 206,296

Paraplegia, I85

Parasites, animal, 148

Paresthesia, I87

Parotitis, 3 I4

Pasteurized milk, 335

Peculiarities of children's diseases, II

Pediculosis capitis, $23^{8}$ 
Pediculosis pubis, 238

Peptonized milk, 339

Peptonizing mixture, 340

Percentage of cream, method for changing, 355

of mixtures, methods of determining, 363

Percussion of heart, 160

Perforation, emergency treatment, 393

in typhoid fever, 260, 265

Pericarditis, 162

Pericardium, 157

Peritonitis, 153

Pernicious anemia, 177

Pertussis, 3 I I

Petit mal, 198

Peyer's patches, 260

Phagocytosis, 420

Pharyngitis, I 3

atrophic II5

chronic, II5

hypertrophic, II5

phlegmonous, 115

Phimosis, 21, 224

Photophobia, 226

Pia, 184

Pica, 103

Piles, 151

Pink-eye, 230

Pitted teeth, I6

Pitting in smallpox, preventing of, 307

Plaster casts, 430

Plethora, 177

Pleurisy, 72 hemorrhagic, 79

Pleurodynia, 79,319

Pleuropneumonia, 96

Pneumohydrothorax, 80

Pneumonia, 84

aspiration, 97

croupous, 20

cry of, 26

cerebral, 96

embolic septic, 98

hypostatic, 97

lobar, 91

treatment of, 98

typhoid, 267

Pneumonic consolidation, 93

Pneumopericardium, I64
Pneumothorax, 80

Poisons and their antidotes, 387

Poliomyelitis, acute anterior, 203

Polydactyly, 24

Polyuria, 2I I, 212

Post-diphtheric paralysis, 206, 296

Pott's disease, 277

Poultices, 399

Premature babies, 34

Pressure symptoms, 193

Prognosis in diseases of children, 27

Proctitis, 151

Prolapse of rectum, $15^{\circ}$ emergency treatment, 394

Promises to child, 45

Proteids, 329

in artificial feeding, $35 \mathrm{I}$

Pseudocrisis in pneumonia, 95

Pseudodiph theria, I 12

Pseudohypertrophy of muscles, 206

Psoas abscess, 279

Pulmonary insufficiency, 169

edema, $7 \mathrm{I}$

emphysema, 69

stenosis, 169

Pulse, 25, 16I

dicrotic, 161

taking of, 37

venous, 162

water-hammer, 162

Purpura, 322

hemorrhagica, 322

Pustules, 236

Pyelitis, 221

QUARANTINE, regulations of, 28

Quinsy, 109

RABIES, 256

Rachitic rosary, 324

Rachitis, 324

Ranula, I03

Rashes, dates when they appear, 244

Rectal irrigation, 42

Rectum, cold compresses to, 42

diseases of, 150

inflammation of, 151

prolapse of, 150 emergency treatment, 394 
Reduplication of heart sounds, I 69

Regurgitation, 167 after feeding, 43

aortic, $\mathrm{r} 68$

mitral, 168

Remittent malarial fever, $25 \mathrm{I}$

Renal calculus, 220 hyperemia, 214

Resolution, stage of, 93

Respiration, 25, 49 artificial, 421

Respiratory failure, emergency treatment, 394 tract, diseases of, 19,46

Rest, 375

Retropharyngeal abscess, I 15

Rhagades, 253

Rheumatism, 22, 317 acute articular, 317 endocarditis after, 2 I complicating, 23

heart murmur in, 20 inflammatory, 317 muscular, 318

Rhinitis, 52

acute, 52

atrophic, 55

chronic, 53

hypertrophic, 55

Rice and oatmeal water, 337

flour gruel, 342

milk, 337

Rickets, 23, 41, 324

Rickety children, eruption of teeth in, 14

Rose cold, 69

Rötheln, 293

Round worms, 149

Rubella, 293

Rubeola, 290

Running ears, 2 I

Rusty sputum, 48

SALT bath, 406 solution, normal, 387 continuous injection, 418

Sarcoma of kidney, 222

Sardonic grin, 255

Scabies, 238

Scales, 236

Scarlatina, 284
Scarlatina miliaris, 286

Scarlet fever, 284

anginoid, 286

kidneys after, 2 r

malignant, 287

Sciatica, 206

Scissors gait, 203

Sclerosis, 203

Scoliosis, 325

Scorbutus, 323

Screw-driver teeth, 16

Scurvy, 23, 323

Seat-worms, 149

Seborrhea, 236

Serum, Flexner's, 247

Shock, emergency treatment, 394

Shower bath, 408

Sight, development of, I4

Singultus, 121

Siphoning milk, apparatus for, 354 method for, 353

Sitting hot-air bath, 406

Sitz bath, 406

Six fingers, 24

Skiagraphy, 435

Skimmed milk, 353

Skin, diseases of, 21, 235 masks for, 434

Sleep, disorders of, 202 in childhood, 34

in infancy, 33

in newborn babe, 32

Smallpox, 304

black, 306

malignant, 306

Smell, development of, I4

Solutions, percentage, rules for, 442

Spasmodic croup, 57

Spasms, nodding, 2 I

Spasmus gyrans, 201

Speech, disorders of, 201

Spice poultice, 400

Spinal cord, anatomy of, 182 diseases of, 202

nerves, I 84

Spine, observation of, $4 \mathrm{I}$

Spitting of blood, treatment, 393

Splint, Hamilton, 426

Splints, 428

Sponge bath, 395, 409

Spotted fever, 245

Sputum, 48 
Sputum in tuberculosis, 283

Starch bath, 408

St. Vitus' dance, 2I, 199

Starch poultice, 400

Stenosis, aortic, I 68

pulmonary, 169

of lacrimal duct, 228

mitral, 167

tricuspid, 168

Sterilization of bottles, 42

of thermometer, 45

Sterilized milk, 336

Stools, disinfection of, in typhoid fever, 269

Stomach, capacity of, 118

dilatation of, 125

diseases of, 118

nursing in, 126

inflammation of, 122

malformations of, I2 I

washing, 414

Stomach-tube, feeding by, 416

Stomatitis, I05

aphthous, I05

gangrenous, I06

parasitic, 106

mercurial, 107

ulcerative, 106

Strabismus, 21,228

Strait jacket, 432

Strangulation of intestine, 147

Strawberry tongue, IOI, 285

Stricture of esophagus, 118

Stridulous breathing, $5^{8}$

Stuttering, 20I

Strumous children, eruption of teeth in, 15

Stye, 228

Subsultus tendinum, 90

Sugar in urine, 214

Sulphur bath, 408

Summer diarrhea, 132

Sunstroke, treatment, 394

Sylvester's method for artificial respiration, 422

Sympathetic nerves, 184

Symptomatology of childrens' diseases, 24

Syncope, 187

Synechia, 228

Syphilis, 253

hereditary, 22
Syphilis, hereditary, cry of, 26

Syphilitic laryngitis, 57

Syringing, 409

ear, 4 Io

eye, 409

mouth, 4I3

nose, 4 II

Syringomyelia, 205

Systole, 167

Systolic sound, I6o

TACHE cérébrale, 247

Tachycardia, 161

Tænia saginata, 148 solium, 148

Talipes valgus, 24 varus, 24

Talking, 14

Tape-worms, 148

Tar bath, 409

Teeth, 14 eruption of, 14 in strumous or rickety children, 15

Hutchinson's, I6

laminated, 16

milk, average age of eruption of, I4

permanent, average age of eruption of, 14,15

pitted, 16

screw-driver, 16

Temper, cry of, 26

Temperature, 24 changing Centigrade to Fahrenheit, 443

methods of reduction, 395

sudden rise of, 43

taking of, 36, $4^{2}$

Tenesmus, 129

Tepid bath, 409

Testicle, undescended, 224

Test-meals, 126

Tetanic convulsions, 185

Tetanus, 255

Tetany, 2I, 200

Therapeutic limit, 378

Therapeutics, 377

Thermometer, sterilization of, 45

Throat, examination of, 43

foreign bodies in, 392 
Thrombosis, 197

Thrush, 106

Tibia, bowing of, 24

Tinea circinata, 238 tonsurans, 238

Tongue in digestive diseases, ror inflammation of, 104 strawberry, Ior

Tongue-tie, 104

Tonsillectomy, I II

Tonsillitis, 108

Tonsils, hypertrophy of, 109

Top-milk mixtures, $35^{8}$

Tormina, 129

Torticollis, 20I, 319

Touch, development of, 14

Tracheotomy, 303

Tremors, 186

Tricuspid stenosis, 168

Trismus, 255

Trousseau's sign, 20I

Tubercular adenitis, 22, 282

arthritis, $28 \mathrm{r}$

dactylitis, 282

laryngitis, 57

meningitis, $2 \mathrm{I}, 190$

osteomyelitis, 28I

Tuberculosis, 22, 27 $\mathrm{r}$

acute miliary, 276

nursing in, 282

of bones, 277

of bronchial lymph-glands, 277

of intestines, $\mathrm{I}_{42}$

of joints, 277

of kidney, 22 $\mathrm{r}$

treatment of, 282

Tuberculous bronchopneumonia, 274

Tumors, cerebral, 197

Turpentine stupe, 398

Tympanites in typhoid fever, 265

Typhoid fever, 22, $25^{8}$

pneumonia, 267

spine, 266

state in bronchopenumonia, 90 ulcer, 260

walking, 262

ULCER, gastric, I 24

of frenum, I04

typhoid, 260
Ulcerative stomatitis, Io6

Uncinaria duodenalis, $15^{\circ}$

Undescended testicle, 224

Urea, I04

Uremia, 215

Urethritis, 225

Urinary tract, diseases of, 209

Urine, 2 I I

collecting of, $2 \mathrm{r} 2$

examination of, 213

method of collecting, 38

retention of, treatment, 395

suppression of, treatment, 394

Urticaria, 238

Uvulitis, I I 5

Vaccination, 308

Vaccines, 420

Vaccinia, 308

Vaginal douching, 420 injections, 420

Vaginitis, 225

Vapor bath, 403

Varicella, 307

Variola, 304

Varioloid, 306

Ventilation, 376

Vesical calculus, 224 spasm, 224

Vesicles, 236

Vincent's angina, II 2

Vinegar and mercury bath, 409

Volvulus, I 47

Vomiting, 120

cyclic, $12 \mathrm{x}$

habit, 121

of blood, treatment, 393

persistent, treatment, 395

Vomitus, disinfection of, in per. tussis, 44

Von Jaksch's disease, 178

WALKING, 14 typhoid, 262

Water, drinking of, $4 \mathrm{I}$

in food, $33 \mathrm{I}$

Water-hammer pulse, 162

Weakness, cry of, 26

Weaning, 349 
Webbed fingers, 24

Weight, I I chart, 13

Griffith's, I2

Weights and measures, 439

Wet cups, 399

Whey, $33^{8}$ and milk, 338 and white of egg, 338 milk, and white of egg, $33^{8}$

Whooping-cough, 3 I I

Widal reaction, 266

Wine measure, table of, 439 whey, 339

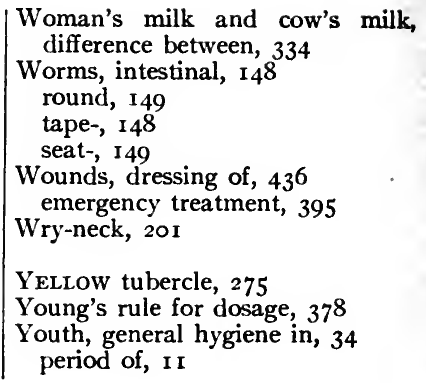




$$
\text { . }
$$<smiles>[CH-]CC</smiles> 


$$
\text { - }
$$




\section{Books for Nurses}

PUBLISHED BY

\section{W. B. SAUNDERS COMPANY \\ West Washington Square \\ Philadelphia}

London: 9, Henrietta Street, Covent Garden

\section{Sanders' Nursing a new work}

Miss Sanders' new book is undoubtedly the most complete and most practical work on nursing ever published. Everything about every subject with which the nurse should be familiar is detailed in a clean cut, definite way. There is no other nursing book so full of good, practical information-information you need.

Modern Methods in Nursing. By Georgiana J. Sanders, formerly Superintendent of Nurses at Massachusetts General Hospital. I2mo of $88 \mathrm{r}$ pages, with 227 illustrations.

Cloth, \$2.50 net.

\section{Aikens' Home Nurse's Handbook}

\section{PRACTICAL}

The point about this work is this: It tells you, and shows you just how to do those little-but none the less important-things entirely omitted from other nursing books, or at best only incidentally treated. The chapters on "Home Treatments" and "Every-Day Care of the Baby," stand out as particularly practical. Then the "Points to be Remembered"-terse, crisp reminders-is a feature of great value.

Home Nurse's Handbook. By CharlotTe A. Aikens, formerly Director of the Sibley Memorial Hospital, Washington, D.C. I2mo of 276 pages, illustrated. Cloth, $\$$ r.5o net 


\section{Stoney's Nursing}

Of this work the American Journal of Nursing says: "It is the fullest and most complete and may well be recommended as being of great general usefulness. The best chapter is the one on observation of symptoms which is very thorough." There are directions how to improvise everything.

Practical Points in Nursing. By EMILY M. A. STONEY, formerly SuperIntendent of the Training School for Nurses in the Carney Hospital, South Boston, Mass. 12mo, 495 pages, illustrated. Cloth, \$r.75 net.

\section{Stoney's Materia Medica}

NEW (3d) EDITION

Stoney's Materia Medica was written by a head nurse who knows just what the nurse needs. American Medicine says it contains "all the information in regards to drugs that a nurse should possess."

Materia Medica for Nurses. By Emıly M. A. Stoney, formerly Superintendent of the Training School for Nurses in the Carney Hospital, South Boston, Mass. I2mo volume of zoo pages. Cloth, $\$$ r.50 net.

\section{Stoney's Surgical Technic}

NEW (3d) EDITION

The first part of the book is dovoted to Bacteriology and Antiseptics; the second part to Surgical Technic, Signs of Death, Bandaging, Care of Infants, etc.

Bacteriology and Surgical Technic for Nurses. By EMILY M. A. Stoney. Revised by Frederic R. Griffith, M. D., New York. $12 \mathrm{mo}$ volume of 3 II pages, fully illustrated. Cloth, \$1.50 net.

\section{Goodnow's First-Year Nursing Just ReAdr}

Miss Goodnow's work deals entirely with the practical side of first-year nursing work. It is the application of text-book knowledge. It tells the nurse how to do those things she is called upon to do in her first year in the training school-the actual ward work.

First-Year Nursing. By MinNie GoOdnow, R. N., formerly SuperIntendent of the Women's Hospital, Denver. 12 mo of 328 pages, illustrated.

Cloth, \$r.50 net. 


\section{Aikens' Hospital Management}

This is just the work for hospital superintendents, trainingschool principals, physicians, and all who are actively interested in hospital administration. The Medical Record says: "Tells in concise form exactly what a hospital should do and how it should be run, from the scrubwoman up to its financing."

Hospital Management. Arranged and edited by Charlotte A. AIKENS, formerly Director o? Sibley Memorial Hospital, Washington, D. C. I2mo of 488 pages, illustrated.

Cloth, $\$ 3.00$ net

\section{Aikens' Primary Studies}

JUST READY NEW' (2d) EDITION

Trained Nurse and Hospital Review says: "It is safe to say that any pupil who has mastered even the major portion of this work would be one of the best prepared first year pupils who ever stood for examination."

Primary Studies for Nurses. By Charlotte A. Aikens, formerly Director of Sibley Memorial Hospital, Washington, D. C. I2mo of 437 pages, illustrated.

Cloth, \$r.75 net.

\section{Aikens' Training-School Methods and the Head Nurse}

This work not only tells how to teach, but also what should be taught the nurse and how much. The Medical Record says: "This book is original, breezy and healthy."

Hospital Training-School Methods and the Head Nurse. By CHARLOTTE A. AlKens, formerly Director of Sibley Memorial Hospital, Washington, D. C. $12 \mathrm{mc}$ of 267 pages.

Cloth, \$r.50 net.

\section{Aikens' Clinical Studies newst ready}

This work for second and third year students is written on the same lines as the author's successful work for primary students. Dietetic and Hygienic Gazette says there "is a large amount of practical information in this book."

Clinical Studies for Nurses. By Charlotte A. Aikens, formerly Director of Sibley Memorial Hospital, Washington, D. C. I2mo of ${ }_{569}$ pages, illustrated

Cloth, $\$ 2.00$ net 


\section{Fowler's Operating Room}

Dr. Fowler's work contains all information of a surgical nature that a nurse must know in order to atto in the highest efficiency. Canadian Joumal of Medicine and Surgery says: "We find compactly and clearly stated just those thousand and one things which when required are so hard to locate."

The Operating Room and the Patient. By RuSSELl S. Fowler, M. D., Professor of Surgery, Brooklyn Postgraduate Medical School. Octavo of 284 pages, with original illust tions. Cloth, $\$ 2.00$ net.

\section{Fiske's The Body}

A NEW IDEA

Trained Nurse and Ilospital Review says "it is concise, wellwritten and well illustrated, and should meet with favor in schools for nurses and with the graduate nurse."

Structure and Functions of the Body. By Annette Fiske, A. M., Graduate of the Waltham Training School for Nurses, Massachusetts. 12 mo of 221 pages, illustrated.

Cloth, \$1.25 net

\section{Beck's Reference Handbook}

NEW (2d) EDITION

This book contains ail the information that a nurse requires to carry out any directions given by the physician. The Montreal Medical Journal says it is " cleverly systematized and shows close observation of the sickroom and hospital regime."

A Reference Handbook for Nurses. By AMANDA K. BECK, Graduate of the Illinois Training School for Nurses, Chicago, Ill. $32 \mathrm{mo}$ volume of 200 pages. Bound in flexible leather, \$r.25 net.

\section{Roberts' Bacteriology \& Pathology $\underset{\text { READY }}{\text { JUST }}$}

This new work is practical in the strictest sense. Written specially for nurses, it confines itself to information that the nurse should know. All unessential matter is excluded. The style is concise and to the point, yet clear and plain. The text is illustrated throughout. 


\title{
DeLee's Obstetrics for Nurses THIRD
}

Dr. DeLee's book really considers two subjects-obstetrics for nurses and actual obstetric nursing. Trained Nurse and Hospital Review says the "book abounds with practical suggestions, and they are given with such clearness that they cannot fail to leave their impress."

Obstetrics for Nurses. By Joseph B. DeLeE, M. D., Professor of Obstetrics at the Northwestern University Medical School, Chicago. r2mo volume of $5^{12}$ pages, fully illustrated. Cloth, \$2.50 net.

\section{Davis' Obstetric \& Gynecologic Nursing}

\author{
THE NEW (3d) EDITION
}

The Trained Nurse and Hospital Review says: "This is one of the most practical and useful books ever presented to the nursing profession." The text is illustrated.

Obstetric and Gynecologic Nursing. By EDWARD P. DAVIS, M. D., Professor of Obstetrics in the Jefferson Medical College, Philadelphia. r.mo volume of 436 pages, illustrated. Buckram, \$r.75 net.

\section{Macfarlane's Gynecology for Nurses}

\section{ILLUSTRATED}

Dr. A. M. Seabrook, Woman's Hospital of Philadelphia, says: "It is a most admirable little book, covering in a concise but attractive way the subject from the nurse's standpoint. You certainly keep up to date in all these matters, and are to be complimented upon your progress and enterprise."

A Reference Handbook of Gynecology for Nurses. By CATHARINE MaCfarlane, M. D., Gynecologist to the Woman's Hospital of Philadelphia. $32 \mathrm{mo}$ of 150 pages, with 70 illustrations. Flexible leather, \$r.25 net.

\section{McKenzie's Exercise in Education and Medicine}

Exercise in Education and Medicine. By R. TAIT McKenzie, B.A., M.D., Professor of Physical Education, and Director of the Department, University of Pennsylvania. Octavo of 406 pages, with 346 illustrations.

Cloth, $\$ 3.50$ net. 


\title{
Manhattan Hospital Eye, Ear, Nose, and Throat Nursing \\ ILLUSTRATED
}

This is a practical book, prepared by surgeons who know the nurse's requirements in these specialties. New York Medical Record says: "Every side of the question has been fully taken into consideration."

Nursing in Diseases of the Eye, Ear, Nose and Throat. By the Committee on Nurses of the Manhattan'Eye, Ear, and Throat Hospltal: J. EDWARD GILES, M. D., Surgeon in Eye Department: ARTHUR B. DUEL, M. D., (chairman), Surgeon in Ear Department; HARMON SMITH, M. D., Surgeon in Throat Department. Assisted by JOHN R. SHANNON, M. D., Assistant Surgeon in Eye Department; and JOHN R. PAGE, M. D., Assistant Surgeon in Ear Department. With chapters by HEKBERT B. WILCOX, M. D., Attending Physician to the Hospital; and Miss EUGENIA D. AYERS, Superintendent of Nurses. 12 mo of 260 pages, illustrated.

Cloth, \$r.5o net.

\section{Friedenwald and Ruhrah's Dietetics for Nurses \\ NEW (2d) EDITION}

This work has been prepared to meet the needs of the nurse, both in training school and after graduation. American Journal of Nursing says it "is exactly the book for which nurses and others have long and vainly sought."

Dietetics for Nurses. By Julius FRIEDENwaLD, M. D., Professor of Diseases of the Stomach, and JOHN RuHRAH, M. D., Professor of Diseases of Children, College of Physicians and Surgeons, Baltimore. 12 mo volume of 395 pages.

Cloth, \$r.50 net

\section{Friedenwald \& Ruhrah on Diet}

THIRD EDITION

Diet in Health and Disease. By Julius FrIEDENWAL.D, M.D., and JohN RUHRAH, M.D. Octavo volume of 764 pages.

Cloth, $\$ 4.00$ net.

\section{Galbraith's Personal Hygiene and Physical Training for Women \\ ILLUSTRATED}

Personal Hygiene and Physical Training for Women. By ANNA $M$. Galbraith, M. D., Fellow New York Academv of Medicine. rmo of $37 \mathrm{r}$ pages, illustrated.

Cloth, \$2.0 net.

\section{Galbraith's Four Epochs of Woman's Life}

\author{
THE NEW (2d) EDITION
}

The Four Epochs of Woman's Life. By ANNA M. GALBRAITh, M.D. With an Introductory Note by JOHN H. MUSSER, M. D., University of Pennsylvania. Izmo of 247 pages.

Cloth, \$r.50 net. 


\section{McCombs' Diseases of Children for Nurses}

NEW (2d) EDITION

Dr. McCombs' experience in lecturing to nurses has enabled him to emphasize just those points that nurses most need to know. National Hospital Record says: "We have needed a good book on children's diseases and this volume admirably fills the want." The nurse's side has been written by head nurses, very valuable being the work of Miss Jennie Manly.

Diseases of Children for Nurses. By ROBERT S. MCCOMBS, M. D., Instructor of Nurses at the Children's Hospital of Philadelphia. I2mo of 470 pages, illustrated.

Cloth, \$2.00 net

\section{Wilson's Obstetric Nursing NEW (2d) EDItION}

In Dr. Wilson's work the entire subject is covered from the beginning of pregnancy, its course, signs, labor, its actual accomplishment, the puerperium and care of the infant. American Journal of Obstetrics says: "Every page empasizes the nurse's relation to the case."

A Reference Handbook of Obstetric Nursing. By W. REynoldS Wilson, M.D., Visiting Physician to the Philadelphia Lying-in Charity. 32mo of 355 pages, illustrated.

Flexible leather, \$r.25 net.

\section{American Pocket Dictionary NEw (7th) EDITION}

The Trained Nurse and Hospital Review says: "We have had many occasions to refer to this dictionary, and in every instance we have found the desired information."

American Pocket Medical Dictionary. Edited by W. A. Newman Dorland, A. M., M. D., Loyola University, Chicago. Flexible leather, gold edges, $\$$ r.oo net; with patent thumb index, $\$$ r.25 net.

\section{Lewis' Anatomy and Physiology $\begin{gathered}\text { SECOND } \\ \text { EDITION }\end{gathered}$}

Nurses Joarnal of Pacific Coast says " it is not in any sense rudimentary, but comprehensive in its treatment of the subjects." The low price makes this book particularly attractive.

Anatomy and Physiology for Nurses. By LEROY Lewis, M.D., Lecturer on Anatomy and Physiology for Nurses, Lewis Hospital, Bay City, Mich. I2mo of 375 pages, 150 illustrations. Cloth, \$x.75 net. 


\section{Boyd's State Registration for Nurses}

State Registration for Nurses. By Loute Croft BoYD, R. N., Grad. uate Colorado Training School for Nurses. Price, 50 cents net.

\section{Paul's Materia Medica}

NEW (2d) EDITION

A Text.Book of Materia Medica for Nurses. By GEORGF P. PAUL, M.D., Samaritan Hospital, Troy, N. Y. I2mo of 282 pages. Cloth, \$1.50 net.

\section{Paul's Fever Nursing}

NEW (2d) EDITION

Nursing in the Acute Infectious Fevers. By George P. PAUL, M.D. $12 \mathrm{mo}$ of 2.46 pages, illustrated. Cloth, \$1.oo net.

\section{Hoxie's Medicine for Nurses}

Practice of Medicine for Nurses. By GeORGe HOWARD HOXIE, M.D., University of Kansas. With a chapter on Technic of Nursing by PEARL L. LAPTAD. 12 mo of 284 pages, illustrated. Cloth, $\$ 1.50$ net.

\section{Grafstrom's Mechano-therapy $\begin{gathered}\text { SECOND } \\ \text { EDITION }\end{gathered}$}

Mechano-therapy (Massage and Medical Gymnastics). By Axel V. GRAFSTROM, B.Sc. M.D., r2mo, 200 page:. Cloth, \$1.25 net.

\section{Nancrede's Anatomy New (7t) EDItion}

Essentials of Anatomy. CHARIES B. G. DENANCREDE, M.D., University of Michigan. r2mo, 4 , pages, r8o illustrations. Cloth, $\$ 1 . \infty$ net.

\section{Morrow's Immediate Care of Injured}

Immediate Care of the Injured. By ALBERT S. MORROW, M.D., New York City Home for Aged and Infirm. Octavo of 354 pages, with 242 fllustrations. Cloth, $\$ 2.50$ net. New (2d) Edition

\section{Register's Fever Nursing}

A Text Book on Practical Fever Nursing. By EdwaRd C. Register, M.D. North Carolina Medical College. Octavo of 350 pages, illus: trated. Cloth, \$2.50 net.

\section{Pyle's Personal Hygiene New (5th) EDItTon}

A Manual of Personal Hygiene. Edited by WAlter L. PYLE, M.D. Wills Eye Hospital, Philadelphia. $12 \mathrm{mo}, 5 \times 5$ pages, illus. \$r.50 net.

\section{Morris' Materia Medica NEw (7th) EDITION}

Essentials of Materia Medica, Therapeutics, and Prescription Writing. By HeNRY MORRIS, M.D. Revised by W. A. BASTEDO, M.D., Columbia University, N. Y. I 2 mo of 300 pages, illustrated. Cloth, $\$$ r.oo net.

\section{Griffith's Care of the Baby NEw (5it) EDITION}

The Care of the Baby. By J. P. Crozer Griffith, M.D., Universily of Pennsylvania. ramo of 455 pages, lllustrated. Cloth, $\$ 1.50$ net. 


$$
\text { - }
$$





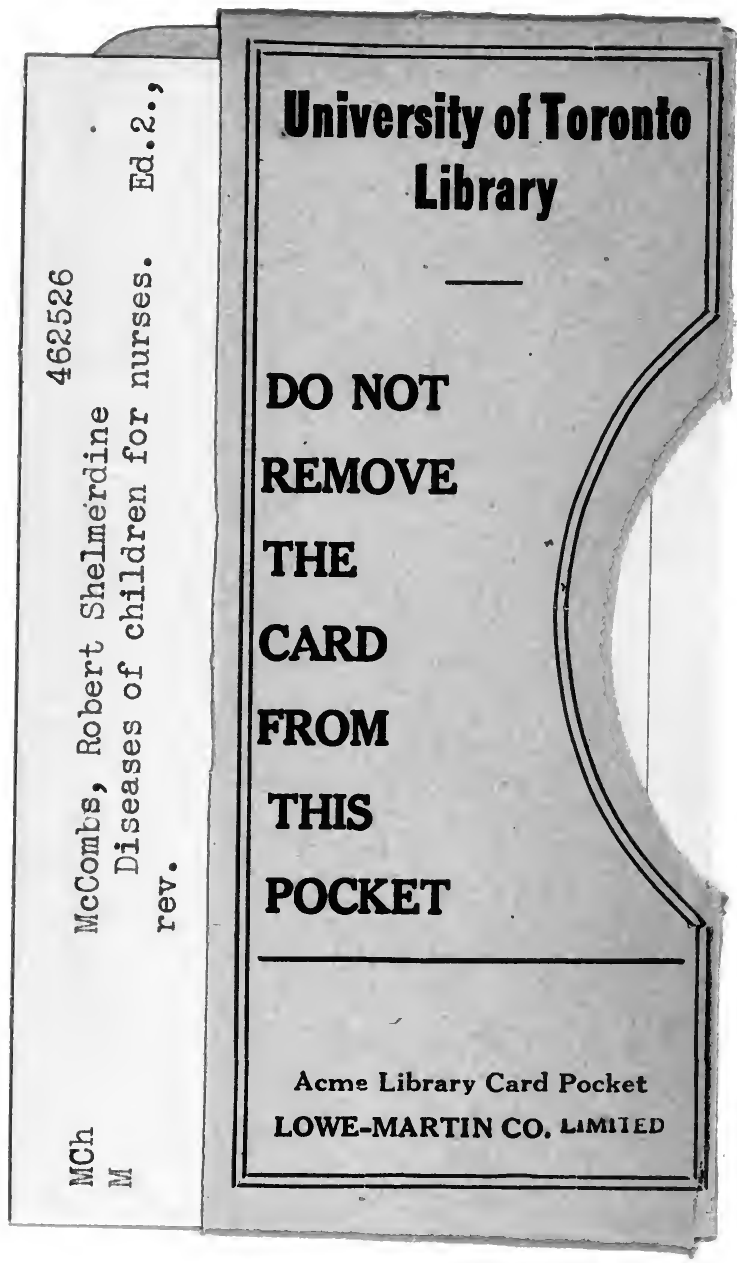


